\title{
Pricing and scheduling under uncertainty
}

Citation for published version (APA):

Marban, S. (2012). Pricing and scheduling under uncertainty. [Doctoral Thesis, Maastricht University]. Datawyse / Universitaire Pers Maastricht. https://doi.org/10.26481/dis.20120608sm

Document status and date:

Published: 01/01/2012

DOI:

$10.26481 /$ dis.20120608sm

Document Version:

Publisher's PDF, also known as Version of record

\section{Please check the document version of this publication:}

- A submitted manuscript is the version of the article upon submission and before peer-review. There can be important differences between the submitted version and the official published version of record.

People interested in the research are advised to contact the author for the final version of the publication, or visit the DOI to the publisher's website.

- The final author version and the galley proof are versions of the publication after peer review.

- The final published version features the final layout of the paper including the volume, issue and page numbers.

Link to publication

\footnotetext{
General rights rights.

- You may freely distribute the URL identifying the publication in the public portal. please follow below link for the End User Agreement:

www.umlib.nl/taverne-license

Take down policy

If you believe that this document breaches copyright please contact us at:

repository@maastrichtuniversity.nl

providing details and we will investigate your claim.
}

Copyright and moral rights for the publications made accessible in the public portal are retained by the authors and/or other copyright owners and it is a condition of accessing publications that users recognise and abide by the legal requirements associated with these

- Users may download and print one copy of any publication from the public portal for the purpose of private study or research.

- You may not further distribute the material or use it for any profit-making activity or commercial gain

If the publication is distributed under the terms of Article $25 \mathrm{fa}$ of the Dutch Copyright Act, indicated by the "Taverne" license above, 


\section{Pricing and Scheduling under Uncertainty}

Sebastián Marbán 
Copyright (c) Sebastián Marbán, Maastricht 2012

All rights reserved. No part of this publication may be reproduced, stored in a retrieval system, or transmitted, in any form, or by any means, electronic, mechanical, photocopying, recording or otherwise, without the prior permission in writing from the author.

Cover illustration: “The Story Machine” by Scott Mccloud

This book was typeset by the author using $\mathrm{HT}_{\mathrm{E}} \mathrm{X}$

Published by Universitaire Pers Maastricht

ISBN 978-94-6159-156-2

Printed in the Netherlands by Datawyse 


\title{
Pricing and Scheduling under Uncertainty
}

\author{
Proefschrift \\ ter verkrijging van de graad van doctor \\ aan de Universiteit Maastricht, \\ op gezag van Rector Magnificus, Prof. mr. G.P.M.F. Mols, \\ volgens het besluit van het College van Decanen, \\ in het openbaar te verdedigen \\ op vrijdag 8 juni 2012 om 14:00 uur
}

door

Sebastián Marbán 


\section{Promotor:}

Prof. dr. ir. C.P.M. van Hoesel

\section{Copromotor:}

Dr. T. Vredeveld

\section{Beoordelingscommissie:}

Prof. dr. A.J. Vermeulen (voorzitter)

Prof. dr. J.R. Correa (Universidad de Chile)

Dr. A. Grigoriev

Prof. dr. F.C.R. Spieksma (K.U. Leuven)

Dit onderzoek werd financieel mogelijk gemaakt door Maastricht Research School of Economics of Technology and Organizations (METEOR). 
Aan Antonio, Gonnie en Carmina. 



\section{Acknowledgements}

This thesis would never have been completed without the help, encouragement, and especially love of many people. Although there are not enough words to describe my gratitude to all of you, I would still like to give it a try.

First of all, I want to thank my supervisor Tjark Vredeveld. Four years ago, his interesting course "Modeling and Solver Technology" convinced me to choose Operations Research as the specialty in my Master of Econometrics and Operations Research. Later that year, his enthusiasm and guidance gave me the opportunity to become a Ph.D. student. It was a long and bumpy road, but you guided me through it and you gave me the freedom to pursue my own ideas. Thank you for that! I am proud that I have been your first Ph.D. student ever, and I wish that many others may follow.

I am also thankful to the members of my assessment committee Dries Vermeulen, José Correa, Alexander Grigoriev, and Frits Spieksma, for their careful reading and constructive comments on the manuscript. A special word of thanks to José, my host advisor at the Departamento de Ingeniería Industrial of the Universidad de Chile, for making my stay in Chile a productive and memorable experience. Furthermore, I am grateful to all my co-authors for their valuable contribution to the research in this thesis. It was a pleasure to work with you all.

Going to the office every day would not have been so pleasant (and probably more productive; hanging around at the secretary office was just too much fun) without two superwomen, Karin van den Boorn and Haydeé Hallmanns. Thank you for your help with my administrative tasks, your advice on female related subjects, and your good humor. Another person I wish to thank for the wonderful working atmosphere is Natalya Usotskaya. Although we were not a perfect match in terms of loudness, I am extremely happy that we were able overcome this and that we get along so well right now. I have said it before, but I will say it again: you were the best officemate I ever had!

Next, I would like to thank my friends and colleagues at the Quantitative Economics department. Bart Diris and Cyriel Rutten, it is an honor to have you as my paranymphs. Bart, thank you for the years of friendship and advice, and for being the best Skype buddy anybody can wish for. If you ever need a friend to go on a horse camp in Germany and share a tent with, you know where to find me. Cyriel, thanks for your patience and great friendship. For many years you have been the stable factor when my mind was on the run again. Also special thanks to Norbert Metiu, Ruben van der Zwaan, and Csapó Gergely, for their true 
friendship and the legendary twenty past three coffee breaks. Our time together gave me the energy to keep on going. Furthermore, I am grateful to the following persons who have become my friends during the course of my Ph.D.: Hansie Ensinck, Birol Yüceoğlu, Hossein Naeemi, Martijn van Brink, José Verschae, Matti Prevoo, Andreea Bicu, Mona Offermanns, Ehsan Vallizadeh, Ron Diris, Thomas Keusch, Nadine Funcke, and Simone Wies. Thank you for making my time in Maastricht so enjoyable.

Besides my friends at work, I would also like to thank the friends from my hometown, Heerlen. Guido, Michiel en Sabel, thank you guys for all the tears of laughter, all the talks about nothing, all the beers we drank, all the great meals we cooked (and Sabel ate), all the security pizza's (that again Sabel ate), all Hertog ice cream we survived together, and overall all your love. May we share it for many more years! Bart Urlings, thank you for more than twenty years of friendship. I hope that many more years full of "Kolonisten van Catan" and "Hotel" games will follow. Moreover, I want to thank Tom Ackermans for all the great nights together in Club Meta, and for simply being a wonderful friend.

Mijn grootste dank gaat uit naar mijn familie. Pap, mam en Carmina, bedankt voor jullie jarenlange steun en liefde. Jullie gaven me de kracht en het zelfvertrouwen om door te gaan. Ik ben super trots dat ik dit proefschrift aan jullie mag opdragen. Joany, heel veel dank voor je onvoorwaardelijke steun, liefde en niet te vergeten time-management. Met jou aan mijn zijde kan ik de hele wereld aan!

Sebastián Marbán

Maastricht, June 2012 


\section{Contents}

1 Introduction $\quad 11$

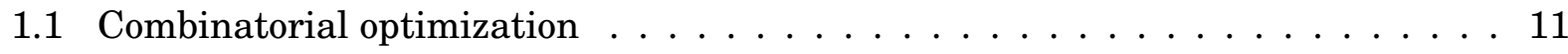

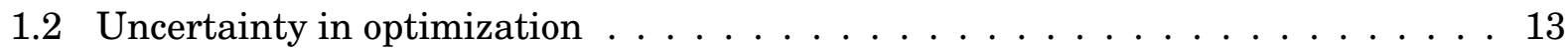

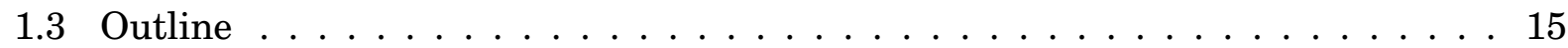

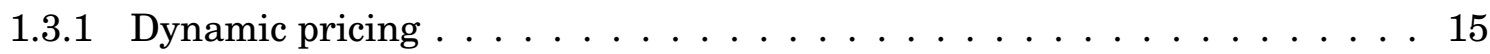

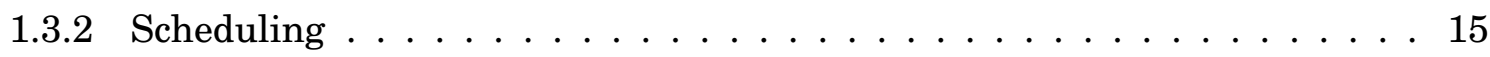

1.4 Publications underlying this thesis $\ldots \ldots \ldots \ldots \ldots \ldots$

2 Dynamic Pricing with Elastic Demand $\quad 19$

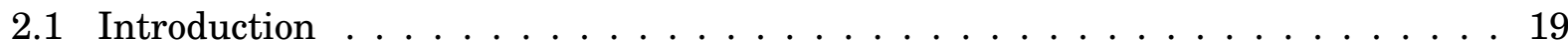

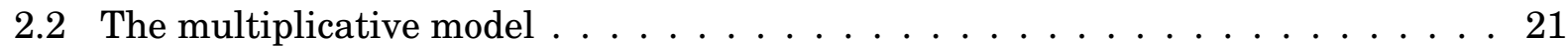

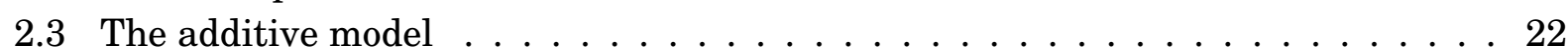

2.3 .1 NP-hardness . . . . . . . . . . . . . . . . . . 22

2.3.2 Approximation scheme . . . . . . . . . . . . . . 27

2.3 .3 A constant number of breakpoints . . . . . . . . . . . . . . . . 29

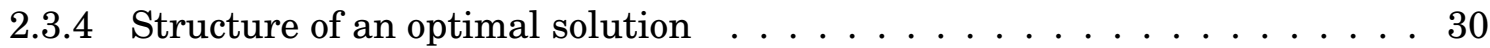

2.4 Concluding remarks . . . . . . . . . . . . . . . . . . 32

3 Scheduling Transmissions: a Game-Theoretic Approach to ALOHA 33

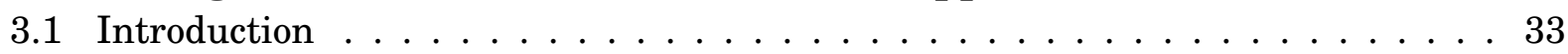

3.2 ALOHA situations . . . . . . . . . . . . . . . . . . 35

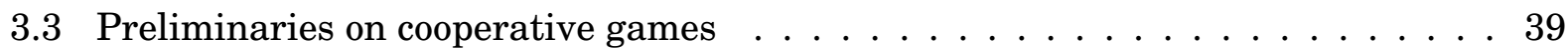

3.4 ALOHA games . . . . . . . . . . . . . . . . . . . . 40

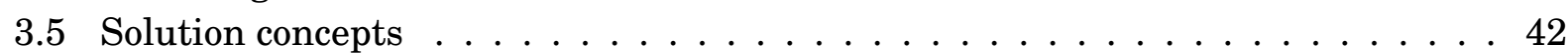

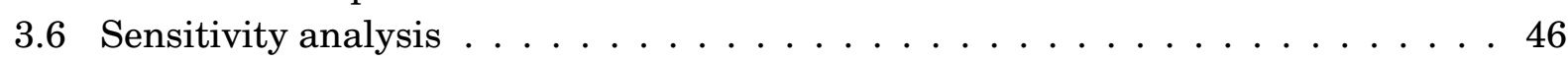

3.7 Concluding remarks . . . . . . . . . . . . . . . . . . . 49

4 The Bayesian Approach to Scheduling $\quad 51$

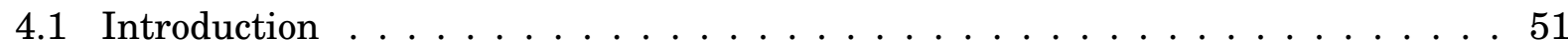

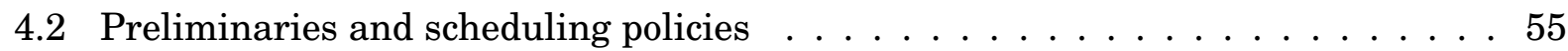

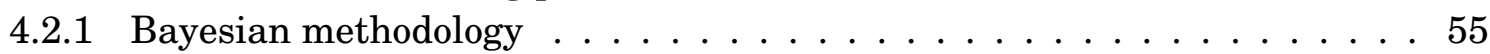

4.2 .2 Bayesian scheduling policies . . . . . . . . . . . . . . 56 


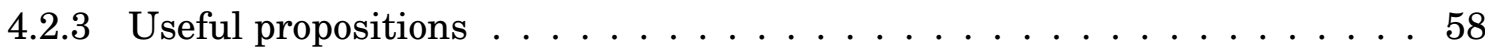

4.3 Problem Setting I . . . . . . . . . . . . . . . . . . . . . 61 61

4.3.1 Upper bound on performance guarantees . . . . . . . . . . . . . 61

4.3.2 Lower bounds on performance guarantees . . . . . . . . . . . . . . . 63

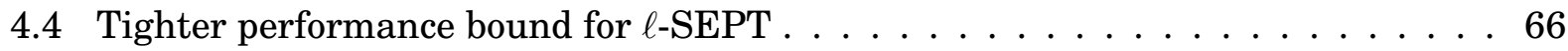

4.5 Problem Setting II . . . . . . . . . . . . . . . . . . . . . . 71

4.5.1 Upper bound on performance guarantees . . . . . . . . . . . . . 71

4.5.2 Tightness of performance guarantees . . . . . . . . . . . . 73

4.6 Problem Setting III . . . . . . . . . . . . . . . . . . . . . . . 74

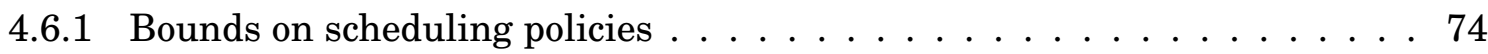

4.6 .2 Structural analysis of OPT . . . . . . . . . . . . . . 76

4.6.3 Upper bound on the performance guarantees . . . . . . . . . . . 78

4.6.4 Tightness of the performance guarantees . . . . . . . . . . . . . 79

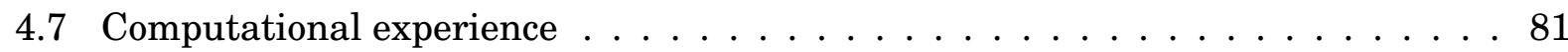

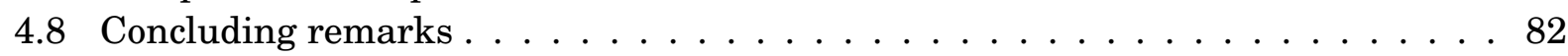

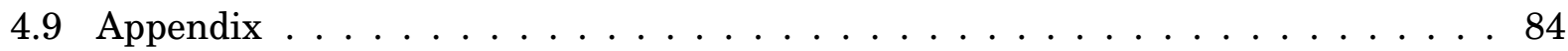

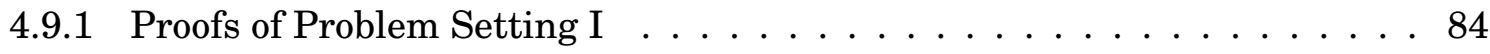

4.9.2 Proofs of Tighter bounds for $\ell$-SEPT . . . . . . . . . . . . . . 89

4.9 .3 Proofs of Problem Setting II _ . . . . . . . . . . . . . . . . 93

4.9 .4 Tables of computational results $\ldots \ldots \ldots \ldots$. . . . . . . . . 97

5 Scheduling with Incomplete Information: an Empirical Evaluation 99

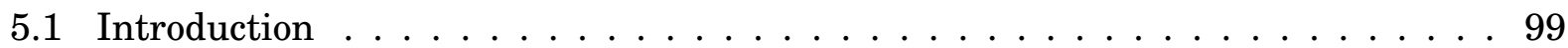

5.2 Scheduling without preemption . . . . . . . . . . . . . . . 101

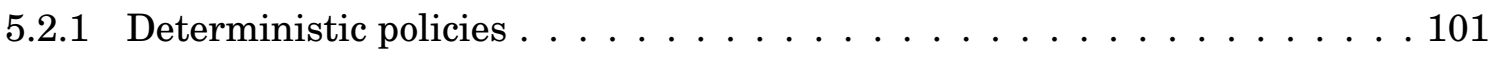

5.2 .2 Randomized policies . . . . . . . . . . . . . . . . . 105

5.3 Scheduling with preemption . . . . . . . . . . . . . . . 108

5.3.1 Learning policies with performance guarantees . . . . . . . . . . . . 109

5.3.2 Learning policies without performance guarantees . . . . . . . . . . . . . 111

5.3 .3 Computational experience . . . . . . . . . . . . . . . 115

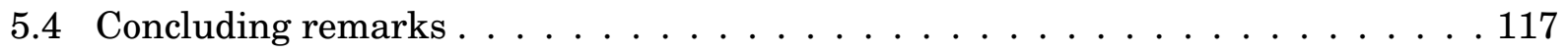

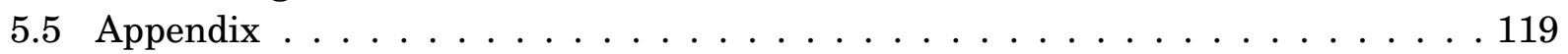

5.5.1 Preliminaries on probability theory . . . . . . . . . . . . . . 119

5.5.2 Figures of computational results . . . . . . . . . . . . . . . 120

$\begin{array}{ll}\text { Bibliography } & 125\end{array}$

$\begin{array}{ll}\text { Nederlandse Samenvatting } & 133\end{array}$

$\begin{array}{lr}\text { Curriculum Vitae } & 137\end{array}$ 


\section{Chapter 1}

\section{Introduction}

"Planning under uncertainty. This, I feel, is the real field that we should all be working in. The real problem is to be able to do planning under uncertainty. The mathematical models that we put together act like we have a precise knowledge about the various things that are happening and we don't. So we need to have plans that actually hedge against these various uncertainties."

— George B. Dantzig, In His Own Voice, 2000 [72].

This thesis deals with several problems in the field of combinatorial optimization under uncertainty. Combinatorial optimization aims at finding the best solution from a finite, or possibly countably infinite, set of feasible solutions. For an extensive overview of combinatorial optimization problems, we refer the reader to the book of Schrijver [100]. In traditional optimization techniques, it is assumed that the decision maker has complete knowledge of all relevant data. As the late George Dantzig, introducer of the simplex method [33], already emphasized in an interview in the year 2000, this assumption is not always realistic. In many situations, we need to find the best solution or make decisions while facing incomplete or even unavailable input data.

In this introductory chapter, we first give a formal description of combinatorial optimization problems in Section 1.1. Next, we explain in Section 1.2 more precisely what we mean by uncertainty, and how it plays a role in the problems discussed in this thesis. Finally, we present an extensive outline of the thesis in Section 1.3, and a list of publications that underly it in Section 1.4.

\subsection{Combinatorial optimization}

A combinatorial optimization problem is described by a set of problem instances, and is either a minimization problem or a maximization problem. Formally, we have the following definition.

Definition 1.1.1. An instance I of a combinatorial optimization problem $\Pi$ consists of two parts:

1. a finite set $S(I)$ of feasible solutions; 
2. a function $f_{\Pi}: S(I) \rightarrow \mathbb{R}$, that assigns to each solution in $S(I)$ a real number.

In case $\Pi$ is a minimization [maximization] problem, an optimal solution for an instance $I$ is a feasible solution $s^{*} \in S(I)$ such that, for all $s \in S(I), f_{\Pi}\left(s^{*}\right) \leq f_{\Pi}\left(s^{*}\right)\left[f_{\Pi}\left(s^{*}\right) \geq f_{\Pi}\left(s^{*}\right)\right]$.

Often the set $S$ of feasible solutions is given implicitly. In the following examples, we present some combinatorial optimization problems, where this is the case. Example 1 is a dynamic pricing problem, which will be discussed in Chapter 2. Examples 2 and 3 are scheduling problems that are studied in Chapter 3 and Chapters 4 and 5, respectively.

Example 1. Consider an online store selling quarterly contracts for computer software. The loyal customer base of the store consists of 100 persons each quarter. Taking into account the software price of 5 euros, this amounts to a total yearly revenue of 2000 euros. Recently, the store appointed a new marketing manager, who proposes the idea of dynamically adjusting the software price over the quarters. Instead of asking 5 euros each quarter, the manager advises to start with a price of 3 euros and increase it every quarter by one euro. Since the customers are not only loyal, but also price-sensitive, this results in quarterly customer bases of $150,135,115$, and 90 persons, and a total revenue of 2105 , which is clearly higher than the revenue with a static price. The question is now if there exists a quarterly dynamic pricing scheme that gives an even higher yearly revenue.

An instance of this problem is given by the number of time periods on which prices need to be set, a range of prices that can be asked, and a demand function that maps a customer base to each price. A solution is given by the prices set at all time periods. The value of a solution is computed by summing up the revenue of each time period, which is the product of the price set and the corresponding customer base.

Example 2. Consider 4 antennas, A, B, C, and D, that each wish to transmit 1 message over a wireless network to a common receiver. Time is divided into intervals, so-called time slots, and at the beginning of each time slot a centralized controller decides which antennas are allowed to transmit. When two or more antennas transmit at the same time slot, the intended receiver may be unable to distinguish between them, and the transmissions fail. Furthermore, each message has a unit time slot length, a value assigned to it, and must be send before a certain deadline otherwise it will be lost. We assume that the vectors of values $v$ and deadlines $d$ of the messages are $v=(3,4,2,5)$ and $d=(2,3,1,3)$. The controller decides to first schedule the transmission of antenna $\mathrm{C}$, followed by those of antennas $\mathrm{A}, \mathrm{B}$, and $\mathrm{D}$. This results in a total value of 9. Since the controller's aim is to maximize the total value of the arrived messages, he would be better off (a total value of 12) by scheduling the transmissions in the following order: A, B, D, and C.

An instance of this problem is specified by the number of antennas and messages, and the corresponding values and deadlines. A solution is represented by an assignment of messages to time slots, and the value of a solution is the sum of values of transmissions that were assigned before their deadline.

Example 3. Consider a set of 6 flat-tire bicycles equally divided over 3 types, A, B, and S. The tires of all bicycles need to be fixed by a single repairman. The time it takes the repairman to fix a tire differs between the types: a type A bicycle takes 4 time units, while type B and $\mathrm{S}$ bicycles take, respectively, 2 and 5 time units. The moment in time at which a bicycle is 
fixed is called its completion time. The goal of the repairman is to fix the tires of all bicycles in such an order that the sum of completion times is minimized. Initially, he works on the bicycle types in alphabetical order, giving a total sum of completion times of 73. After doing so, the repairman realizes that it would be much smarter to first process the bicycles of type $\mathrm{B}$, followed by types $\mathrm{A}$ and $\mathrm{S}$. This results in a total sum of completion times of 65 , which is substantially lower.

An instance of this problem is given by the number of repairmen, the number of bicycle types, the number of flat-tire bicycles belonging to each of these types, and the amount of time it takes a repairman to fix a flat-tire. A solution is specified by the assignment of bicycles to repairman over time. The value of a solution is the sum of all times at which a repairman finishes fixing the tire of a bicycle.

For many combinatorial optimization problems it is difficult to find the optimal solution in a reasonable amount of time. For this reason, a lot of research in the field of combinatorial optimization focuses on developing algorithms that try to find a "good" solution instead of the best solution. These algorithms are referred to as approximation algorithms, and are formally defined as follows.

Definition 1.1.2. An algorithm $A$ is an approximation algorithm for combinatorial optimization problem $\Pi$ if, given any instance $I$, it finds a feasible solution $s \in S(I)$.

Some approximation algorithms are designed to give a (theoretical) guarantee on the quality of the obtained solution, while others just focus on obtaining good practical performance. Let the value $f_{\Pi}(s)$ of feasible solution $s \in S(I)$ found by algorithm $A$ when applied to $I$ be denoted by $A(I)$, and the value of the optimal solution $s^{*} \in S(I)$ by $\mathrm{OPT}(I)$.

Definition 1.1.3. An algorithm $A$ is called a $\rho$-approximation algorithm if, for each instance I of a minimization [maximization] problem, we have that $A(I) \leq \rho \cdot O P T(I)$ with $\rho \geq 1$ $[A(I) \geq \rho \cdot \operatorname{OPT}(I)$ with $\rho \leq 1]$. We refer to $\rho$ as the (worst case) performance guarantee of algorithm A.

\subsection{Uncertainty in optimization}

Optimization under uncertainty has been actively studied over the last several decades. Starting from the pioneering papers of a.o. Bellman [13], Bellman and Zadeh [12], and Dantzig [34] it continues to be a topic that stimulates new research, see [29, 37, 85]. Problems in the field are characterized by the necessity of making decisions in the face of the unknown, that is, while having incomplete information about the input data or the possible consequences of decisions made. There is a wide variety of approaches to model the lack of information in optimization problems.

In one of these approaches, the uncertainty is modeled by assuming that part of the data is given in terms of probability distributions. This model, called stochastic optimization and introduced by Beale [10] and Dantzig [34], is studied in Chapters 2, 4 and 5. The first of these chapters, Chapter 2, is dedicated to a dynamic pricing problem for a company that sells a product to a group of potential customers, who only buy if the price does not exceed their reservation price, i.e., the maximum price they are willing to pay. This reservation price is 
uncertain to the company, and it is modeled as a random variable with a certain probability distribution. The goal of the company is to set its prices over time such that expected revenue is maximized.

In the other two chapters, we consider stochastic machine scheduling problems, in which the processing times of jobs are random variables for which the parameters of the underlying distribution are known. When the objective is to minimize the sum of expected completion times, the Shortest Expected Processing Time (SEPT) rule is optimal for the non-preemptive (Rothkopf [94]) single machine version of this problem, and also for the preemptive one with exponentially distributed processing times (Pinedo [87]). One may wonder, however, how realistic it is to assume complete knowledge of the parameter values in these problems. Motivated by this insight, we distinguish ourselves from the traditional stochastic scheduling literature by introducing additional uncertainty on the parameters of the processing time distributions. In Chapter 4, the scheduler still has some initial beliefs about the true parameter values, while in Chapter 5 the scheduler is completely in the dark.

Chapter 3 concludes this thesis by considering another approach to uncertainty in optimization. We study a wireless network in which each node for itself decides how to schedule its transmissions. In designing a wireless network, it is important to schedule transmissions in such a way that network performance is optimized [19, 23, 32, 102]. This is especially the case in a distributed network, such as wireless LAN, in which each node for itself decides when to transmit. This lack of coordination leads to uncertainty with respect to the arrival of transmissions. Coordination among the nodes, however, results in improved network performance, and the question is how to allocate the corresponding gains.

In many real-life situations, it is indeed the case that we do not have full knowledge over what is happening, which could lead to "wrong" decision making. For this reason, it is important to study how we can possibly deal with uncertainty. In the chapters above, we propose three techniques to do so in a systematic way: a dynamic programming approach for the pricing problem in Chapter 2; a probabilistic updating scheme for the parameters of the processing time distributions in the machine scheduling problem of Chapter 4; and a trialand-error method for the same parameters in the machine scheduling problem in Chapter 5. This latter method uses an initial scheduling fase in which it learns with a few jobs such that more informed decisions can be made later on.

Although the techniques in Chapters 4 and 5 have been used in the field of operations research before, their application to stochastic scheduling problems is truly novel. This is rather surprising since the repetitive nature of scheduling problems poses great opportunities for learning. Moreover, in a scheduling setting more precise knowledge of the input can make a crucial difference between a "good" and a "bad" schedule, and learning has the potential to provide this information. For these reasons, learning techniques should be a key practical and theoretical consideration in any uncertain machine scheduling setting. This thesis attempts to be the first consistent work on this topic. 


\subsection{Outline}

\subsubsection{Dynamic pricing}

Until a few decades ago, dynamic pricing methods were mainly considered to be a tool within the field of revenue management. The original literature of this field is build upon the pioneering work of Belobaba [14, 15], Littlewood [70], and Rothstein [95, 96]. Over the last few decades, dynamic pricing has received considerable attention from other research areas, like theoretical computer science, e.g., by Guruswami, Hartline, Karlin, and Kempe [52], and its applications spread out to many other industries, see the work of Bitran and Caldentey [18] and Weatherford and Bodily [112]. This is explained by swift developments in information technology, e-commerce, and Internet, which enabled companies to collect extensive amounts of customer data, and to use this to quickly update their prices if necessary, at almost no cost [24]. Nowadays, dynamic pricing is an active field of research - some examples of recent papers are given by Popescu and Wu [89] and Farias and Van Roy [43].

In Chapter 2, we contribute to the pricing literature by analyzing the computational complexity of a dynamic pricing problem. This area of research has received attention only recently, see the papers of Akcay, Natarajan, and Xu [2] and Berger, Grigoriev, and Van Loon [17]. We consider a company that sells a single product to a group of customers over a finite time horizon. These customers are price sensitive and the price of today influences the group of customers of tomorrow. Furthermore, the customers only buy a unit of the product if the price is below their reservation price, which has a certain probability distribution. The objective is to set the prices over time so as to maximize expected revenue. Two pricesensitivity models are studied: multiplicative and additive demand change. For the first model, we present a polynomial time algorithm that solves it to optimality. However, the second model turns out to be significantly more difficult to solve. We give a pseudo-polynomial time algorithm, and prove the complementary result that the problem is weakly NP-hard. Moreover, we present an Fully Polynomial Time Approximation Scheme (FPTAS), which is an algorithm that, for any $\epsilon>0$, is guaranteed to find a solution whose expected revenue is within a factor $(1+\epsilon)$ of the optimal expected revenue, in time polynomial in the input and $1 / \epsilon$.

\subsubsection{Scheduling}

According to the famous Oxford English Dictionary (OED), scheduling stands for the act of 'planning an event to take place at a particular time.' A more specific formulation defines scheduling to be the method by which jobs, processes or data flows are given access to system resources in such a way that some given objective is optimized. Scheduling plays an vital role within the area of telecommunications, where it is, for example, used to schedule transmission of communication requests in a way that avoids interference of concurrent transmissions and minimizes the total time needed to successfully schedule all requests [80]. Scheduling is also an important tool for manufacturing and computer science, where it can have a major impact on the efficiency of production and computation processes, see the handbook of scheduling by Leung [66]. Several of the problems that arise in this field are known as machine scheduling problems. We distinguish between deterministic and stochastic machine scheduling. In the 
former problem, all input data is known in advance with certainty, while in the latter one the processing times (or other parts of the input data) are susceptible to random fluctuations.

Scheduling of transmissions. A wireless network refers to any type of computer network in which nodes send their transmissions through air using radio waves. Starting off as academic curiosities [1, 60, 108], they have become an important part of our every-day life over the last few decades. An elaborate definition and introduction to wireless network communication can be found in the books of Pahlavan and Krishnamurthy [84] and Stallings [107]. In a wireless network, transmissions may be scheduled through a centralized controller or by using a distributed mechanism. In the distributed case, each node decides for itself when to transmit, and a so-called Medium Access Control (MAC) protocol is used to regulate transmissions between the nodes. The earliest example of such a MAC protocol is ALOHA, introduced by Abramson [1]. The idea behind this protocol is as follows: a node transmits if there is data available to send. When this transmission fails, the node tries to retransmit the data after some random number of time.

In Chapter 3, we study a wireless network in which transmissions between nodes are scheduled by a refined variant of the ALOHA protocol, developed by Roberts [91]. When two nodes using this protocol transmit a signal at the same time, interference may occur resulting in lost transmissions. Cooperation between the nodes can circumvent the mutual interference, thereby having a positive impact on the network performance. In order to study how the performance gains obtained from this cooperation can be allocated among the nodes, we define and analyze a cooperative game, called an ALOHA game. In addition, we develop a set of rules that allocate the gains from cooperation among the nodes. These rules are referred to as Weighted Gain Splitting (WGS), since they attempt to balance out the individual contribution of each of node to the performance gains. Finally, we study three solution concepts from cooperative game theory that are applied as allocation rules in the ALOHA game: the core (introduced by Gillies [46]), the Shapley value (introduced by Shapley [103]), and the compromise value (introduced by Tijs [109]).

Machine scheduling. Stochastic scheduling has been an active field of research for more than 40 years, see the book of Pinedo [88] and the dissertations of Dean [35] and Uetz [111]. In Chapter 4, we study a stochastic scheduling problem in which several classes of independent jobs have to be processed by a single machine. The processing times of the jobs are assumed to be exponentially distributed with unknown parameters depending on the class of each job. The objective is to minimize the sum of expected completion times. A Bayesian framework is adopted in which the parameter uncertainty is modeled by probability distributions on the unknown parameters. Although Bayesian techniques are widely applied in operations research $[6,65]$, there are only few papers on this topic in the field of stochastic scheduling. An example is the paper of Hamada and Glazebrook [53], which shows that solving instances of the Bayesian scheduling problem to optimality is highly impractical, if not impossible. This calls for the need to develop policies of low computational effort which yield good qualitative performance.

The Shortest Expected Processing Time (SEPT) policy and its adaptive variant, referred to as learning-SEPT ( $\ell$-SEPT), are such policies. Their performance is studied in the Bayesian 
framework for three different problem settings without preemption, i.e., once a job started it needs to be processed until completion. For the setting with $m$ different job classes, we show that the objective value of SEPT is at most a factor $m$ away from the optimal policy's value, and we construct instances for which this bound is tight. Furthermore, we prove that $\ell$-SEPT is no worse than SEPT, and has a smaller lower bound of $1+\sqrt{m-1}$ on the performance guarantee. We conjecture that this is the correct performance guarantee for $\ell$-SEPT. For the problem settings with two job classes, either with two unknown parameters or with one known and one unknown parameter, we provide a tight performance guarantee that is strictly smaller than 2. Finally, we give computational results showing that $\ell$-SEPT empirically outperforms SEPT.

In Chapter 5, we consider again the stochastic single machine scheduling problem to minimize the total sum of expected completion times. We focus on two job classes and assume that the scheduler has no prior beliefs about the parameter values, which is different from the Bayesian scheduling approach of the previous chapter. Overall, the scheduler thus lacks all knowledge about the jobs of the classes, except for knowing that each job is drawn from some specific exponential distribution and knowing the exact processing time of a job when it is completed. We could therefore also speak of a non-clairvoyant stochastic scheduling problem. Pioneered by Motwani, Phillips, and Torng [81], there is nowadays a rich literature on non-clairvoyant scheduling. Most of these papers consider an online problem, in which jobs become available to the scheduler over time. To overcome bad competitive ratios (for a definition see [44]), it is custom in this area of research to give back an advantage to the non-clairvoyant scheduler [40]. In our problem, this advantage is given by the fact that the optimal policy faces uncertainty with respect to the processing times (even though it knows the correct parameter values).

For the scheduling problem without preemption, we introduce deterministic and randomized policies, also referred to as Class Comparison (CC) policies. The basic idea behind these policies is to first schedule a single job of each class, compare the resulting processing times, and continue by processing the jobs of the class corresponding to the faster job followed by the jobs of the other class. It turns out that each of the CC policies gives a tight performance guarantee of at most 2 . For the problem with preemption, in which jobs may be interrupted from processing and resumed later on, we subsequently conjecture that there is a constant lower bound of 1.1010 on the performance guarantee of all possible scheduling policies. In addition, we provide a tight performance guarantee for the EQUI policy [110], which partitions the machine evenly among the unfinished jobs and preempts only when jobs are completed. Thereafter, we introduce several other policies for which we do not have any performance guarantees, and we compare the computational performance of all proposed policies. We find the best performance guarantees, very close to the conjectured lower bound, for a policy based on the Proportional Sharing algorithm [28].

\subsection{Publications underlying this thesis}

The results of this thesis are based on the following set of publications. The work is done in close cooperation with the respective co-authors.

1. S. Marbán, G.R.J. Van der Zwaan, A. Grigoriev, B. Hiller, and T. Vredeveld. Dynamic 
Pricing Problems with Elastic Demand. Operations Research Letters, 40 (3): pp. 175179, 2012.

2. S. Marbán, P. Van de Ven, P. Borm, and H. Hamers. A Cooperative Game-Theoretic Approach to ALOHA. METEOR Research Memorandum, RM/10/049, Maastricht University, School of Business and Economics, 2010.

3. S. Marbán, C. Rutten, and T. Vredeveld. Tight performance in Bayesian Scheduling. METEOR Research Memorandum, RM/10/052, Maastricht University, School of Business and Economics, 2010.

4. S. Marbán, C. Rutten, and T. Vredeveld. Learning in Stochastic Machine Scheduling. In Proceedings of the 9th Workshop on Approximation and Online Algorithms (WAOA 2011), Lecture Notes in Computer Science, 7164, Springer, pp. 21-34, 2011.

5. S. Marbán, C. Rutten, and T. Vredeveld. Bayesian Scheduling with Multiple Job Classes. Working paper, Maastricht University, School of Business and Economics, 2012.

6. J.R. Correa, S. Marbán, J. Verschae. Single Machine Scheduling with Incomplete Information. Working paper, Maastricht University, School of Business and Economics, 2012 . 


\section{Chapter 2}

\section{Dynamic Pricing with Elastic Demand $^{1}$}

\subsection{Introduction}

In this chapter we consider a dynamic pricing problem for a company that sells a single product to a group of customers over a finite time horizon. These customers are price sensitive and the price of today influences the group of customers of tomorrow. The objective is to set the prices over time so as to maximize revenue. We develop algorithms that solve several variants of this problem to optimality. Further, we show that one variant is NP-hard, present a pseudo-polynomial time algorithm, and transform this into a fully polynomial time approximation scheme (FPTAS), which is an algorithm that, for any $\epsilon>0$, is guaranteed to find a solution whose expected revenue is within a factor $(1+\epsilon)$ of the optimal expected revenue, in time polynomial in the input and $1 / \epsilon$.

Problem Definition. We consider the following dynamic pricing problem. For a finite time horizon of $T$ periods, a company needs to determine prices for a single product available in unlimited supply. Periods are indexed consecutively from 0 to $T$. At each time period $t$, there are $C_{t}$ customers for the product. Each of these customers buys a unit of the product only if the price, denoted by $\pi_{t}$, does not exceed the customer's reservation price. Let $\pi=\left(\pi_{0}, \ldots, \pi_{T-1}\right)$ denote the vector of all prices asked. It is assumed that the reservation prices for customers present at time $t$ are independent and identically distributed and that the probability distributions are given as part of the input. Let $F_{t}($.$) denote the cumulative probability distribution$ of the reservation price of a customer at time period $t$. The probability that a customer buys a unit of the product at time period $t$ is then given by $\bar{F}_{t}\left(\pi_{t}\right)=1-F_{t}\left(\pi_{t}\right)$.

We assume that the customers are price sensitive and the price of today influences the number of customers of tomorrow. Let $C_{0}$ be the initial number of customers. Two models of elastic demand are studied. In the first model, referred to as multiplicative model, we assume that the number of customers at time $t$ changes by a price-dependent multiplicative term

$$
C_{t}(\pi)=\left(1+S_{t-1}\left(\pi_{t-1}\right)\right) \cdot C_{t-1}(\pi) .
$$

\footnotetext{
${ }^{1}$ This chapter is based on Marbán, Van der Zwaan, Grigoriev, Hiller, and Vredeveld (2011).
} 


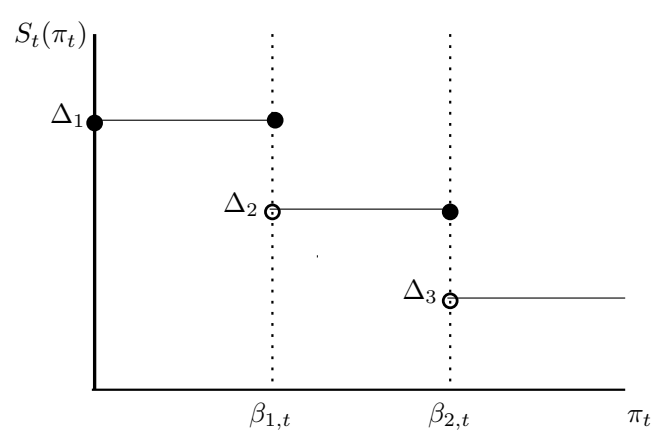

Figure 2.1: A step function at time period $t$ with $k=3$

For every time period $t \in\{0, \ldots, T-1\}, S_{t}\left(\pi_{t}\right): \mathbb{R}_{+} \rightarrow\left\{\Delta_{1}, \ldots, \Delta_{k}\right\}$ with $\Delta_{1} \geq \ldots \geq \Delta_{k}>-1$ is a non-increasing step-function defined by $S_{t}\left(\pi_{t}\right)=\Delta_{i}$ if $\pi_{t} \in I_{i, t}$, where

$$
I_{i, t}= \begin{cases}{\left[0, \beta_{1, t}\right]} & \text { if } i=1, \\ \left(\beta_{k-1, t}, \infty\right) & \text { if } i=k, \\ \left(\beta_{i-1, t}, \beta_{i, t}\right] & \text { otherwise }\end{cases}
$$

with given values $0 \leq \beta_{1, t} \leq \ldots \leq \beta_{k-1, t}<\infty$. See Figure 2.1 for an example with three levels of change.

In the second model, referred to as additive model, we assume that the number of customers at time $t$ changes by an additive term

$$
C_{t}(\pi)=C_{t-1}(\pi)+S_{t-1}\left(\pi_{t-1}\right)
$$

where $S_{t}\left(\pi_{t}\right)$ is defined as above, but $\Delta_{i} \in\left\{-C_{0}, \ldots, C_{0}\right\}$.

Price levels that bring the total number of customers below zero are not allowed, so $C_{t} \geq 0$ for every $t \in\{0, \ldots, T\}$. In what follows, we will refer to $\Delta_{i}$ as level of change $i$ in the number of customers and to $\beta_{i, t}$ as breakpoint $i$ of step function $S_{t}\left(\pi_{t}\right)$. Observe that the levels of change are the same for all time periods, whereas the breakpoints may change between time periods. Furthermore, we use $C_{t}$ instead of $C_{t}(\pi)$ whenever $\pi$ is clear from the context.

The problem is to set the prices over the time horizon so as to maximize the total expected revenue. Formally,

$$
\max _{\pi}\{\mathbb{E}[R(\pi)]\}=\max _{\pi}\left\{\sum_{t=0}^{T-1} \pi_{t} \cdot C_{t}(\pi) \cdot \bar{F}_{t}\left(\pi_{t}\right)\right\},
$$

where $R(\pi): \mathbb{R}_{+}^{T} \rightarrow \mathbb{R}_{+}$denotes the total revenue given price vector $\pi=\left(\pi_{0}, \ldots, \pi_{T-1}\right)$. Throughout this chapter, an optimal solution to the dynamic pricing problem is denoted by $\pi^{*}=\left(\pi_{0}^{*}, \ldots, \pi_{T-1}^{*}\right)$.

For every $i \in\{1, \ldots, k\}$ and $t \in\{0, \ldots, T-1\}$, let $p_{i, t}$ denote the price $\pi_{t} \in I_{i, t}$ that maximizes $\pi_{t} \cdot \bar{F}_{t}\left(\pi_{t}\right)$ : the expected revenue per customer at time $t$ for level of change $i$. We refer to this price as level price. It is easy to see that an optimal solution to the dynamic pricing problem consists only of level prices. For ease of notation, we define $\bar{p}_{i, t}=p_{i, t} \cdot \bar{F}_{t}\left(p_{i, t}\right)$ to be the expected revenue per customer by asking level price $p_{i, t}$, for each $i$ and $t$. Moreover, we assume that the maximum expected revenue per customer and the corresponding level price are returned by an oracle in constant time. For this reason, we omit the corresponding factor from the running time analysis, and count it as an elementary operation. 
Previous work. Dynamic pricing is an increasingly active field of research. An extensive overview of achievements in the field can be found in [18, 24, 112]. The problem considered in the chapter is embedded in the existing literature as follows. As in Kopalle, Rao, and Assunção [62] and Monahan, Petruzzi, and Zhao [79], we focus on a discrete time model. In reality, companies are unable to change their prices continuously, and therefore the continuous time model is impractical. In line with Chan, Simchi-Levi, and Swann [25] and Maglaras and Meissner [73], we further study price-setting companies either having monopolistic market power or operating in a market with imperfect competition. The same product setting can be found in, among others, Popescu and Wu [89]. Finally, demand is determined by multiplying the number of potential customers by the probability that the price asked is below the customer's reservation price such that the product is bought. This demand setting is similar to Lin [69] and Zhao and Zheng [116].

This chapter contributes to the pricing literature by analyzing the computational complexity of dynamic pricing problems. This area of research has received attention only recently. For example, Akcay, Natarajan, and Xu [2] develop a polynomial-time algorithm for a dynamic pricing problem of a company that sells multiple substitutable products. Berger, Grigoriev, and Van Loon [17] present another example. They develop a polynomial-time algorithm for a company that wishes to gradually implement new prices for a set of different products. Esmaeilsabzali and Day [42] study a pricing problem for web service providers with finite capacity. For different variants, they give NP-hardness proofs, and propose online algorithms. As a last example, Akhlaghpour, Ghodsi, Haghpanah, Mirrokni, and Nikzad [3] discuss the pricing of digital products in order to maximize revenue, when the value of such products is a function of the set of buyers who have already bought them. For several problem settings, they give an inapproximability result, develop approximation algorithms, and a fully polynomial-time approximation scheme (FPTAS).

Our contribution. Our main contribution is the thorough exploration of a dynamic pricing problem in which the number of customers of today depends on all pricing decisions of the past. We consider two different customer models: a multiplicative and an additive model. For the first model, we present in Section 2.2 a polynomial time algorithm that solves it to optimality. However, the second model turns out to be significantly more difficult to solve. In Section 2.3, we give a pseudo-polynomial time algorithm, and prove the complementary result that the problem is weakly NP-hard. Further, we present an FPTAS, which is a non-trivial transformation of the pseudo-polynomial time algorithm. Finally, we give an algorithm that runs in polynomial time when there is a constant number of breakpoints, and we show that the optimal solution has an interesting structure in case the reservation price distributions and breakpoints stay constant over time.

\subsection{The multiplicative model}

In this section, we study the dynamic pricing problem under multiplicative demand change. In order to solve the problem to optimality, we propose a dynamic programming algorithm that is denoted in the proof of the following theorem. 
Theorem 2.2.1. An optimal solution to the dynamic pricing problem with a multiplicative model can be found in $O(k \cdot T)$ time and $O(1)$ space.

Proof. Given $C_{t}$ customers at time period $t$, let $V_{t}\left(C_{t}\right)$ denote the maximum expected revenue obtained between time periods $t$ and $T$,

$$
V_{t}\left(C_{t}\right)=\max _{i \in\{1, \ldots, k\}}\left\{V_{t+1}\left(\left(1+S_{t}\left(p_{i, t}\right)\right) \cdot C_{t}\right)+\bar{p}_{i, t} \cdot C_{t}\right\}
$$

for all $t \in\{0, \ldots, T-1\}$, and $V_{T}\left(C_{T}\right)=0$. Further, define

$$
A_{t}=\max _{i \in\{1, \ldots, k\}}\left\{\left(1+S_{t}\left(p_{i, t}\right)\right) \cdot A_{t+1}+\bar{p}_{i, t}\right\}
$$

for all $t \in\{0, \ldots, T-1\}$, and $A_{T}=0$.

We show that $V_{t}\left(C_{t}\right)=A_{t} \cdot C_{t}$ for all $t \leq T-1$, by induction on $t$. The base case $V_{T-1}\left(C_{T-1}\right)=$ $A_{T-1} \cdot C_{T-1}$ is easy to verify. Now, suppose for some $t$, we have that $V_{t+1}\left(C_{t+1}\right)=A_{t+1} \cdot C_{t+1}$. Then,

$$
\begin{aligned}
V_{t}\left(C_{t}\right) & =\max _{i \in\{1, \ldots, k\}}\left\{V_{t+1}\left(\left(1+S_{t}\left(p_{i, t}\right)\right) \cdot C_{t}\right)+\bar{p}_{i, t} \cdot C_{t}\right\} \\
& =\max _{i \in\{1, \ldots, k\}}\left\{\left(1+S_{t}\left(p_{i, t}\right)\right) \cdot C_{t} \cdot A_{t+1}+\bar{p}_{i, t} \cdot C_{t}\right\} \\
& =\max _{i \in\{1, \ldots, k\}}\left\{\left(1+S_{t}\left(p_{i, t}\right)\right) \cdot A_{t+1}+\bar{p}_{i, t}\right\} C_{t} \\
& =A_{t} \cdot C_{t},
\end{aligned}
$$

where the second equality follows from the induction step.

Hence, in order to obtain the maximum expected revenue, it is sufficient to compute $A_{0}$ and multiply it by $C_{0}$. For the running time, notice that for each $A_{t}, t \in\{0, \ldots, T-1\}$, we need to find the maximum over $k$ values. Each of these values takes constant time to compute, and therefore the total computation time is $O(k \cdot T)$. Moreover, for time period $t$ we only need the value $A_{t+1}$ to compute $A_{t}$ such that the total space requirement is constant.

\subsection{The additive model}

In this section, the dynamic pricing problem under additive demand change is studied. In contrast to the problem in the previous section, this problem turns out to be much harder to solve. We prove that the problem is weakly NP-hard, and that there is a pseudo-polynomial time algorithm, which is transformed into an FPTAS. Finally, we give a polynomial time algorithm for the case with a constant number of breakpoints, and we show that the optimal solution has an interesting structured property in case the reservation price distributions and breakpoints remain constant over time.

\subsubsection{NP-hardness}

In this subsection, we reduce the well-known weakly NP-hard problem KNAPSACK [45] to the dynamic pricing problem with an additive model. Let an instance of knapsack be given by items $0, \ldots, n-1$ with values $v_{0}, \ldots, v_{n-1}$, weights $w_{0}, \ldots, w_{n-1}$, and knapsack capacity $W$. 


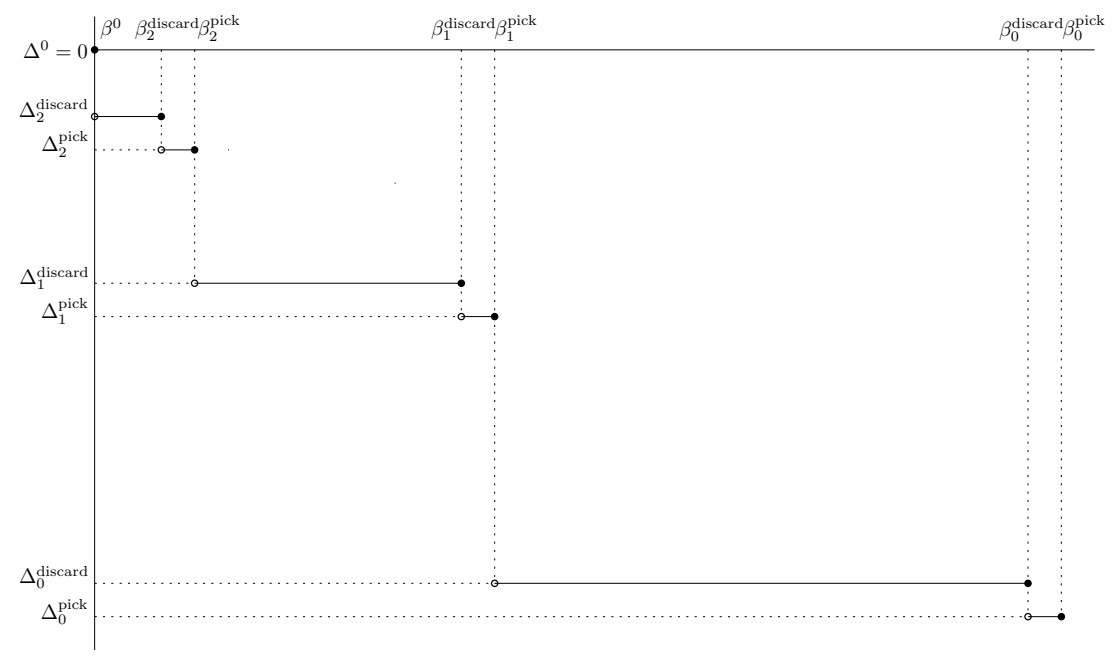

Figure 2.2: Breakpoints and levels of change for the NP-hardness proof

The objective is to find a subset of the items of the highest total value such that their total weight does not exceed the capacity. For notational ease, define $v_{\max }=\max _{i \in\{0, \ldots, n-1\}} v_{i}$ and $w_{\max }=\max _{i \in\{0, \ldots, n-1\}} w_{i}$. Without loss of generality, we assume that $w_{\max } \leq W$.

We construct an instance of the dynamic pricing problem from KNAPSACK as follows. Let $N \geq 6 n+\left\lceil\log v_{\max }\right\rceil+\lceil\log W\rceil$ be a large number. Further, set the number of time periods to $T=n$, define $L_{i}=\sum_{j=0}^{i} 2^{j+3 N}=2^{3 N}\left(2^{i+1}-1\right)$ for every $i \in\{0, \ldots, n-1\}$ and $L_{-1}=0$, and let $C_{0}=L_{n-1}+W$. We create for every item $i \in\{0, \ldots, n-1\}$ two levels of change:

$$
\Delta_{i}^{\text {pick }}=-2^{n-1-i+3 N}-w_{i} \quad \text { and } \quad \Delta_{i}^{\text {discard }}=-2^{n-1-i+3 N},
$$

with corresponding breakpoints

$$
\beta_{i}^{\text {pick }}=2^{4(n-1-i)+N}+\frac{v_{i} \cdot 2^{2 N}}{L_{n-1-i}} \quad \text { and } \quad \beta_{i}^{\text {discard }}=2^{4(n-1-i)+N},
$$

which remain constant over time. In addition, we create a zero change $\Delta^{0}=0$ whenever a price of zero is asked, i.e., $\beta^{0}=0$, for every time period $t \in\{0, \ldots, T-1\}$. See Figure 2.2 for an example with $n=3$. We assume that the cumulative distribution function of the customers' reservation price $F_{t}(\cdot)$ is such that the level prices are equal to the created breakpoints and the customers always buy a unit of the product at every level price. In the remainder of this subsection, we therefore denote the level prices by $\beta_{i}^{\text {pick }}$ and $\beta_{i}^{\text {discard }}$, and we have that $\bar{F}_{t}\left(\beta_{i}^{\text {pick }}\right)=\bar{F}_{t}\left(\beta_{i}^{\text {discard }}\right)=1$ for all $i$, and $\bar{F}_{t}\left(\pi_{t}\right)=0$ for all $\pi_{t}>\beta_{0}^{\text {pick }}$, for every time period $t$. Notice that this instance can be constructed in polynomial time.

Intuitively, choosing a price equal to $\beta_{i}^{\text {pick }}$ and $\beta_{i}^{\text {discard }}$ corresponds to either picking item $i$ in the knapsack or discarding the item. We will show below that the way we set our prices and levels of change forces an optimal solution to ask precisely one of the prices, $\beta_{i}^{\text {pick }}$ or $\beta_{i}^{\text {discard }}$, for every item $i$. First, we present two preliminary results. The first one states that the non-zero prices in the optimal solution are decreasing over time.

Lemma 2.3.1. Let $\pi^{*}=\left(\pi_{0}^{*}, \ldots, \pi_{T-1}^{*}\right)$ be an optimal price vector. If $\pi_{t}^{*}, \pi_{s}^{*}>0$ for $t<s \leq T-1$ and $\pi_{r}^{*}=0$ for all $t<r<s$, then $\pi_{t}^{*} \geq \pi_{s}^{*}>0$. 
Proof. In case a zero price is present in a solution to the dynamic pricing problem, it is clear that the total expected revenue is independent of the time at which the zero price is chosen. Zero prices can therefore be moved to the end of the time horizon. Hence, without loss of generality we assume that $s=t+1$.

Suppose by contradiction that $0<\pi_{t}^{*}<\pi_{t+1}^{*}$ for some $t$. Let $\delta_{t}^{*}$ and $\delta_{t+1}^{*}$ be the levels of change corresponding to the prices $\pi_{t}^{*}$ and $\pi_{t+1}^{*}$. Then, the total expected revenue obtained at times $t$ and $t+1$ is

$$
C_{t} \cdot \pi_{t}^{*}+\left(C_{t}+\delta_{t}^{*}\right) \cdot \pi_{t+1}^{*}=C_{t} \cdot \pi_{t}^{*}+C_{t} \cdot \pi_{t+1}^{*}+\delta_{t}^{*} \cdot \pi_{t+1}^{*} .
$$

By exchanging prices $\pi_{t}^{*}$ and $\pi_{t+1}^{*}$, we obtain a total expected revenue of

$$
C_{t} \cdot \pi_{t+1}^{*}+\left(C_{t}+\delta_{t+1}^{*}\right) \cdot \pi_{t}^{*}=C_{t} \cdot \pi_{t+1}^{*}+C_{t} \cdot \pi_{t}^{*}+\delta_{t+1}^{*} \cdot \pi_{t}^{*} .
$$

Note that the number of customers at time $t+2$ is not affected by this exchange.

By construction, we know that for some integers $0 \leq \tau_{1} \leq \tau_{2} \leq n-1$,

$$
\begin{gathered}
2^{4 \tau_{1}+N} \leq \pi_{t}^{*} \leq 2^{4 \tau_{1}+N}+\frac{v_{\max } \cdot 2^{2 N}}{L_{\tau_{1}}} \\
-2^{\tau_{1}+3 N} \geq \delta_{t}^{*} \geq-2^{\tau_{1}+3 N}-w_{\max }
\end{gathered}
$$

and

$$
\begin{gathered}
2^{4 \tau_{2}+N} \leq \pi_{t+1}^{*} \leq 2^{4 \tau_{2}+N}+\frac{v_{\max } \cdot 2^{2 N}}{L_{\tau_{2}}} \\
-2^{\tau_{2}+3 N} \geq \delta_{t+1}^{*} \geq-2^{\tau_{2}+3 N}-w_{\max }
\end{gathered}
$$

Combining (2.3) and (2.4) gives an upper bound for $\delta_{t}^{*} \pi_{t+1}^{*}$,

$$
\delta_{t}^{*} \cdot \pi_{t+1}^{*} \leq-2^{\tau_{1}+3 N} \cdot 2^{4 \tau_{2}+N}=-2^{\tau_{1}+4 \tau_{2}+4 N} .
$$

Further, a lower bound for $\delta_{t+1}^{*} \pi_{t}^{*}$ is obtained by combining (2.2) and (2.5),

$$
\begin{aligned}
\delta_{t+1}^{*} \cdot \pi_{t}^{*} & \geq\left(-2^{\tau_{2}+3 N}-w_{\max }\right)\left(2^{4 \tau_{1}+N}+\frac{v_{\max } \cdot 2^{2 N}}{L_{\tau_{2}}}\right) \\
& >\left(-2^{\tau_{2}+3 N}-2^{N}\right)\left(2^{4 \tau_{1}+N}+\frac{v_{\max } \cdot 2^{2 N}}{L_{\tau_{2}}}\right) \\
& >-2^{\tau_{2}+3 N+1} \cdot\left(2^{4 \tau_{1}+N}+\frac{v_{\max } \cdot 2^{2 N}}{L_{\tau_{2}}}\right) \\
& >-2^{\tau_{2}+3 N+1} \cdot\left(2^{4 \tau_{1}+N}+1\right) \\
& >-2^{4 \tau_{1}+\tau_{2}+4 N+2}
\end{aligned}
$$

where the second inequality is due to the definition of $N$, the third inequality follows as $2^{\tau_{2}+3 N}>2^{N}$, and the fourth inequality follows from $v_{\text {max }} \cdot 2^{2 N}<2^{3 N} \leq L_{\tau_{2}}$, again by definition of $N$.

Finally, combining (2.6) and (2.7) brings us to

$$
\delta_{t}^{*} \cdot \pi_{t+1}^{*} \leq-2^{\tau_{1}+4 \tau_{2}+4 N}<-2^{4 \tau_{1}+\tau_{2}+4 N+2} \leq \delta_{t+1}^{*} \cdot \pi_{t}^{*} .
$$

Thus,

$$
C_{t} \cdot \pi_{t}^{*}+C_{t} \cdot \pi_{t+1}^{*}+\delta_{t}^{*} \cdot \pi_{t+1}^{*}<C_{t} \cdot \pi_{t+1}^{*}+C_{t} \cdot \pi_{t}^{*}+\delta_{t+1}^{*} \cdot \pi_{t}^{*},
$$

which contradicts the fact that $\pi^{*}$ is optimal. 
The next lemma implies that in the optimal solution $\pi^{*}$ for every $i$ either $\beta_{i}^{\text {pick }}$ or $\beta_{i}^{\text {discard }}$ or none of these is chosen.

Lemma 2.3.2. Let $\pi^{*}=\left(\pi_{0}^{*}, \ldots, \pi_{T-1}^{*}\right)$ be an optimal price vector. For all $t \in\{0, \ldots, T-2\}$, there does not exist an integer $\tau \in\{1, \ldots, T-1\}$ such that

$$
2^{4 \tau+N} \leq \pi_{t+1}^{*} \leq \pi_{t}^{*} \leq 2^{4 \tau+N}+\frac{v_{\max } \cdot 2^{2 N}}{L_{\tau}}
$$

Proof. Suppose by contradiction that $2^{4 \tau+N} \leq \pi_{t+1}^{*} \leq \pi_{t}^{*} \leq 2^{4 \tau+N}+\frac{v_{\max } \cdot 2^{2 N}}{L_{\tau}}$ for some $\tau \in$ $\{1, \ldots, T-1\}$. Let $\delta_{t}^{*}$ and $\delta_{t+1}^{*}$ again denote the levels of change corresponding to the prices $\pi_{t}^{*}$ and $\pi_{t+1}^{*}$. By construction, we then know that for $s \in\{t, t+1\}$

$$
-2^{\tau+3 N} \geq \delta_{s}^{*} \geq-2^{\tau+3 N}-w_{\max }
$$

such that the total expected revenue obtained at times $t$ and $t+1$ is at most

$$
\left(C_{t}+\left(C_{t}+\delta_{t}^{*}\right)\right) \cdot\left(2^{4 \tau+N}+\frac{v_{\max } \cdot 2^{2 N}}{L_{\tau}}\right)<2 \cdot C_{t} \cdot 2^{4 \tau+N+1},
$$

where the inequality follows from the fact that $\delta_{t}^{*} \leq 0$ for all $t$, and from $v_{\max } \cdot 2^{2 N}<L_{\tau}$ by the definition of $N$ and $L_{\tau}$.

However, the total expected revenue obtained by replacing the price at time $t$ by $\beta_{n-1-(\tau+1)}^{\text {discard }}$ and asking a zero price at time $t+1$ is at least

$$
C_{t} \cdot 2^{4(\tau+1)+N}=2 C_{t} \cdot 2^{4 \tau+N+3},
$$

which is strictly larger than $2 C_{t} \cdot 2^{4 \tau+N+1}$, the total expected revenue obtained before the replacement. In addition, the change in the number of customers is

$$
\delta_{t}^{*}+\delta_{t+1}^{*} \leq-2^{\tau+3 N} \cdot 2=\Delta_{n-1-(\tau+1)}^{\text {discard }}
$$

before the replacement, and $-2^{\tau+3 N+1}$ afterwards.

Hence, by replacing the prices we end up with at least as many customers as before, and a strictly larger expected revenue. This contradicts the fact that $\pi^{*}$ is optimal.

Lemma 2.3.3. Let $\pi^{*}=\left(\pi_{0}^{*}, \ldots, \pi_{T-1}^{*}\right)$ be an optimal price vector. Then, $\pi_{t}^{*}$ is equal to either $\beta_{t}^{\text {discard }}$ or $\beta_{t}^{\text {pick }}$ for all time periods $t \in\{0, \ldots, T-1\}$.

Proof. From Lemma 2.3.1, it follows that prices in the optimal solution $\pi^{*}$ are monotone decreasing, and therefore if $\beta_{i}^{\text {pick }}$ and $\beta_{i}^{\ell}$, with $\ell \in\{$ pick, discard $\}$, or two times $\beta_{i}^{\text {discard }}$ are asked, they are subsequent. However, Lemma 2.3.2 states that $\beta_{i}^{\ell_{1}}$ and $\beta_{i}^{\ell_{2}}$ with $\ell_{1}, \ell_{2} \in$ \{pick, discard $\}$ are never asked subsequently, for all $i \in\{1, \ldots, n-1\}$. Besides, we cannot ask price $\beta_{0}^{\text {discard }}$ or $\beta_{0}^{\text {pick }}$ twice, nor any combination of the two, since $2 \cdot \Delta_{0}^{\text {discard }}>C_{0}$. This proves the lemma in case there are no zero prices in the optimal solution. If zero prices are present, there exists a smallest index $k$ such that neither $\beta_{k}^{\text {pick }}$ nor $\beta_{k}^{\text {discard }}$ is chosen. 
Let us adjust the optimal solution $\pi^{*}$ by replacing $\beta_{j}^{\text {pick }}$ by $\beta_{j}^{\text {discard }}$ for all possible $j$. Thereby, the loss in expected revenue is at most

$$
\begin{aligned}
n \cdot C_{0} \cdot \max _{l<n}\left\{\left(\beta_{l}^{\text {pick }}-\beta_{l}^{\text {discard }}\right)\right\} & =n \cdot\left(L_{n-1}+W\right) \cdot \frac{v_{\max } \cdot 2^{2 N}}{L_{0}} \\
& <2^{n} \cdot\left(2^{n+3 N}+2^{N}\right) \\
& <2^{2 n+3 N+1}
\end{aligned}
$$

where the first inequality follows from $v_{\max } \cdot 2^{2 N}<2^{3 N}=L_{0}$ and $W<2^{N}$ by definition of $N$.

Next, shift prices from and including time step $k$ to the right one step, and move the last

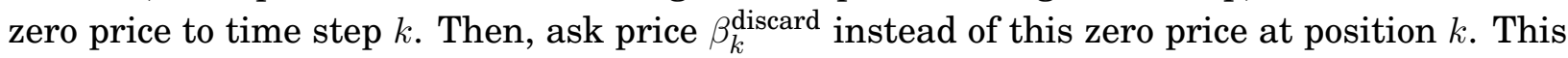
gives a valid price vector, because by construction, there exists a feasible solution consisting of picking all discard prices. As a result, the number of customers drops by $\left|\Delta_{k}^{\text {discard }}\right|$ for the remaining time steps, which results in a total loss of expected revenue of at most

$$
\begin{aligned}
\left|\Delta_{k}^{\text {discard }}\right| \cdot \sum_{j=k+1}^{n-1} \beta_{j}^{\text {discard }} & =2^{n-1-k+3 N} \cdot \sum_{j=k+1}^{n-1} 2^{4(n-1-j)+N} \\
& <2^{5(n-1-k)+4 N-3} .
\end{aligned}
$$

By the adjustments to the optimal solution proposed above, we further gain at least

$$
\begin{aligned}
\left(C_{0}+\sum_{j=0}^{k-1} \Delta_{j}^{\text {discard }}\right) \cdot \beta_{k}^{\text {discard }} & \geq\left(L_{n-1}-\sum_{j=0}^{k-1} 2^{n-1-j+3 N}\right) \cdot \beta_{k}^{\text {discard }} \\
& =L_{n-1-k} \cdot 2^{4(n-1-k)+N} \\
& \geq 2^{5(n-1-k)+4 N} .
\end{aligned}
$$

Combining (2.8) and (2.9) and comparing to (2.10), we finally find that the expected revenue gain of the adjustments is larger than the loss,

$$
2^{2 n+3 N+1}+2^{5(n-1-k)+4 N-3}<2 \cdot 2^{5(n-1-k)+4 N-3}<2^{5(n-1-k)+4 N} .
$$

This is a contradiction to the fact that $\pi^{*}$ is optimal. Hence, no zero prices are asked in an optimal solution, and at each time period $t$ we pick at most one price from $\left\{\beta_{t}^{\text {pick }}, \beta_{t}^{\text {discard }}\right\}$.

This brings us to the following theorem.

Theorem 2.3.4. The dynamic pricing problem with an additive model is NP-hard.

Proof. We construct a dynamic pricing instance from KNAPSACK as described in the beginning of the section. By construction, every valid knapsack solution has a corresponding valid solution to the dynamic pricing problem. Furthermore, we show below that an optimal dynamic pricing solution has a corresponding optimal knapsack solution.

From Lemma 2.3.3, we know that $\pi_{t}^{*} \in\left\{\beta_{t}^{\text {pick }}, \beta_{t}^{\text {discard }}\right\}$ at time period $t \in\{0, \ldots, T-1\}$. Let $x_{i}$ denote a binary variable indicating whether price $\beta_{i}^{\text {pick }}$ or $\beta_{i}^{\text {discard }}$ is chosen, for all $i \in\{0, \ldots, n-1\}: x_{i}=1$ if we choose $\beta_{i}^{\text {pick }}$, and $x_{i}=0$ otherwise. Further, let $\rho_{t-1}=W-$ $\sum_{j=0}^{t-1} x_{j} \cdot w_{j}$ 
The number of customers at time period $t$ is

$$
\begin{aligned}
C_{t} & =C_{0}+\sum_{j=0}^{t-1} \Delta_{j}^{\text {discard }}-\sum_{j=0}^{t-1} x_{j} \cdot w_{j} \\
& =C_{0}-\sum_{j=0}^{t-1} 2^{n-1-j+3 N}-\sum_{j=0}^{t-1} x_{j} \cdot w_{j} \\
& =L_{n-1-t}+\rho_{t-1} .
\end{aligned}
$$

Note that $C_{T}=\rho_{T-1} \geq 0$ by the fact that the number of customers is always non-negative. Therefore, $\sum_{j=0}^{t-1} x_{j} \cdot w_{j} \leq W$ and $\left(x_{0}, \ldots, x_{n-1}\right)$ is a feasible knapsack solution. Then, the total expected revenue is given by

$$
\begin{aligned}
\mathbb{E}[R & \left.\left.\pi_{0}^{*}, \ldots, \pi_{T-1}^{*}\right)\right]=\sum_{t=0}^{T-1} \pi_{t}^{*} \cdot C_{t} \\
= & \sum_{i=0}^{n-1}\left[x_{i} \cdot\left(2^{4(n-1-i)+N}+\frac{v_{i} \cdot 2^{2 N}}{L_{n-1-i}}\right)\right. \\
& \left.+\left(1-x_{i}\right) \cdot 2^{4(n-1-i)+N}\right] \cdot\left(L_{n-1-i}+\rho_{i-1}\right) \\
= & \sum_{i=0}^{n-1} 2^{4(n-1-i)+N} \cdot L_{n-1-i}+\sum_{i=0}^{n-1} 2^{4(n-1-i)+N} \cdot \rho_{i-1} \\
& +\sum_{i=0}^{n-1} x_{i} \cdot v_{i} \cdot 2^{2 N}+\sum_{i=0}^{n-1} x_{i} \frac{v_{i} \cdot 2^{2 N}}{L_{n-1-i}} \rho_{i-1} .
\end{aligned}
$$

The term $\sum_{i=0}^{n-1} x_{i} \cdot v_{i} \cdot 2^{2 N}$ is the total value of KNAPSACK, multiplied by $2^{2 N}$. Note that $\sum_{i=0}^{n-1} 2^{4(n-1-i)+N} \cdot L_{n-1-i}$ is independent of the prices chosen. Furthermore, $\sum_{i=0}^{n-1} 2^{4(n-1-i)+N}$. $\rho_{i-1}$ and $\sum_{i=0}^{n-1} x_{i} \frac{v_{i} \cdot 2^{2 N}}{L_{n-1-i}} \rho_{i-1}$ are dominated by $\sum_{i=0}^{n-1} x_{i} \cdot v_{i} \cdot 2^{2 N}$. This follows from the fact that it can be assumed without loss of generality that at least one item is chosen in the knapsack, such that the latter number is at least $2^{2 N}$ and the first two numbers are at most $n \cdot 2^{4 n+N+\log W}$ and $n \cdot 2^{\log W}$, respectively. By dividing by $2^{2 N}$ and rounding down we obtain the total value of $\left(x_{0}, \ldots, x_{n-1}\right)$ in the corresponding KNAPSACK instance. Hence, if price vector $\pi^{*}$, with corresponding $\left(x_{0}, \ldots, x_{n-1}\right)$, maximizes expected revenue, then $\left(x_{0}, \ldots, x_{n-1}\right)$ also maximizes the value for the KNAPSACK instance.

\subsubsection{Approximation scheme}

In this subsection, we present a pseudo-polynomial time dynamic programming algorithm that solves the dynamic pricing problem under additive demand change. Further, we transform this algorithm into an FPTAS.

We use the following notation. Let $\Delta_{\max }=\max _{i \in\{1, \ldots, k\}}\left\{\Delta_{i}\right\}=\Delta_{1}$. An upper bound on the expected revenue per customer, $\bar{p}_{\text {max }, t}$, that can be obtained in time period $t$ is denoted by

$$
\bar{p}_{\text {max }, t}=\max \left\{\bar{p}_{i, t} \mid C_{0}+t \cdot \Delta_{\max }+\Delta_{i} \geq 0 \text { for } i \in\{1, \ldots, k\}\right\} .
$$


Furthermore, we define an upper and lower bound on the optimal expected revenue,

$$
R_{\max }=\sum_{t=0}^{T-1}\left(C_{0}+t \cdot \Delta_{\max }\right) \cdot \bar{p}_{\max , t} \geq \mathbb{E}\left[R\left(\pi^{*}\right)\right]
$$

and

$$
R_{\min }=\max _{t \leq T-1}\left\{\left(C_{0}+t \cdot \Delta_{\max }\right) \cdot \bar{p}_{\max , t}\right\} \leq \mathbb{E}\left[R\left(\pi^{*}\right)\right],
$$

where $\pi^{*}$ is an optimal price vector. Note that $R_{\max } \leq T \cdot R_{\min }$.

Theorem 2.3.5. An optimal solution to the dynamic pricing problem with an additive model can be found in $O\left(k \cdot T \cdot R_{\max }^{2}\right)$ time.

Proof. Let $C(t, r)$ denote the maximum number of customers at time period $t$ such that the total expected revenue until this time period is at least $r$. Further, let

$$
\begin{aligned}
W_{t, r}=\left\{\left(i, r^{\prime}\right) \mid\right. & C\left(t-1, r^{\prime}\right) \cdot \bar{p}_{i, t}+r^{\prime} \geq r \\
& \text { and } C\left(t-1, r^{\prime}\right)+\Delta_{i} \geq 0 \\
& \text { for } \left.i \in\{1, \ldots, k\} \text { and } r^{\prime} \leq r\right\}
\end{aligned}
$$

be the set of tuples $\left(i, r^{\prime}\right)$ such that asking level price $p_{i, t}$ when having expected revenue $r^{\prime}$ yields an expected revenue of at least $r$, and the number of customers stays non-negative.

The recursive formula for the update of the values $C(t, r)$ in the dynamic programming algorithm reads

$$
C(t, r)= \begin{cases}-\infty & \text { if } W_{t, r}=\emptyset \\ \max _{\left(i, r^{\prime}\right) \in W_{t, r}}\left\{C\left(t-1, r^{\prime}\right)+\Delta_{i}\right\} & \text { otherwise }\end{cases}
$$

for all time periods $t \in\{1, \ldots, T\}$ and

$$
C(0, r)= \begin{cases}C_{0} & \text { if } r=0 \\ -\infty & \text { otherwise. }\end{cases}
$$

Then, the maximum expected revenue equals

$$
\mathbb{E}\left[R\left(\pi^{*}\right)\right]=\max \{r \mid C(T, r) \geq 0\} .
$$

Correctness of this dynamic programming algorithm follows from a straightforward induction argument. Moreover, the time complexity is as follows. We need to compute at most $R_{\max }$ values at time period $t$. Each of these values takes at most $O\left(k \cdot R_{\max }\right)$ time to obtain by expression (2.13). Hence, the total computation time is $O\left(k \cdot T \cdot R_{\max }^{2}\right)$.

The idea for the FPTAS is now to make the computation of the pseudo-polynomial time dynamic programming algorithm efficient by reducing the number of distinct expected revenue values $r$ for which $C(t, r)$ is computed at each time period $t$.

Theorem 2.3.6. For any $\epsilon>0$, the dynamic pricing problem with an additive model admits a $O\left(k \cdot T^{5} / \epsilon^{2}\right)$ time $(1-\epsilon)$-approximation. 
Proof. Given $\epsilon>0$, let $K=\frac{\epsilon \cdot R_{\min }}{T+1}$. We adapt the recursive formulation in Theorem 2.3 .5 of $C(t, r)$ to $\tilde{C}(t, r)$ as follows: replace $W_{t, r}$ by $W_{t, r} \cap\left\{\left(i, r^{\prime}\right) \mid r^{\prime}\right.$ is a multiple of $\left.K\right\}$. Further, only compute $\tilde{C}(t, r)$ when $r$ is a multiple of $K$.

Fix an optimal price vector $\pi^{*}$ with expected revenue sequence $r_{0}, \ldots, r_{T}$, where $r_{t}$ denotes the expected revenue obtained up to time period $t$. Moreover, let $\tilde{r}_{t}=K \cdot\left\lfloor r_{t} / K\right\rfloor-K \cdot t$ for all $t \in\{0, \ldots, T\}$. We claim that $C\left(t, r_{t}\right) \leq \tilde{C}\left(t, \tilde{r}_{t}\right)$ for all $t \in\{0, \ldots, T\}$. For the base case, with $t=0$, the claim follows trivially. Suppose for some $t$ that $C\left(s, r_{s}\right) \leq \tilde{C}\left(s, \tilde{r}_{s}\right)$ for all $s \leq t$. Then,

$$
\begin{aligned}
\pi_{t}^{*} \cdot \tilde{C}\left(t, \tilde{r}_{t}\right) \cdot \bar{F}_{t}\left(\pi_{t}^{*}\right)+\tilde{r}_{t} & \geq \pi_{t}^{*} \cdot C\left(t, r_{t}\right) \cdot \bar{F}_{t}\left(\pi_{t}^{*}\right)+\tilde{r}_{t} \\
& =\pi_{t}^{*} \cdot C\left(t, r_{t}\right) \cdot \bar{F}_{t}\left(\pi_{t}^{*}\right)+K \cdot\left\lfloor r_{t} / K\right\rfloor-K \cdot t \\
& \geq \pi_{t}^{*} \cdot C\left(t, r_{t}\right) \cdot \bar{F}_{t}\left(\pi_{t}^{*}\right)+r_{t}-K \cdot(t+1) \\
& =r_{t+1}-K \cdot(t+1) \geq \tilde{r}_{t+1},
\end{aligned}
$$

where the first line follows from the induction hypothesis, the second equality by definition of $r_{t+1}$, and the last inequality from $\tilde{r}_{t+1}=K \cdot\left\lfloor r_{t+1} / K\right\rfloor-K \cdot(t+1) \leq r_{t+1}-K \cdot(t+1)$. By the induction hypothesis and (2.14),

$$
\tilde{C}\left(t+1, \tilde{r}_{t+1}\right) \geq \tilde{C}\left(t, \tilde{r}_{t}\right)+S_{t}\left(\pi_{t}^{*}\right) \geq C\left(t, r_{t}\right)+S_{t}\left(\pi_{t}^{*}\right)=C\left(t+1, r_{t+1}\right) .
$$

Let $\tilde{\pi}$ be an optimal price vector for the adapted dynamic program. Then,

$$
\mathbb{E}[R(\tilde{\pi})] \geq \mathbb{E}\left[R\left(\pi^{*}\right)\right]-K \cdot(T+1)=\mathbb{E}\left[R\left(\pi^{*}\right)\right]-\epsilon \cdot R_{\min } \geq(1-\epsilon) \mathbb{E}\left[R\left(\pi^{*}\right)\right],
$$

where the first inequality follows from our claim $C\left(T, r_{T}\right) \leq \tilde{C}\left(T, K \cdot\left\lfloor r_{T} / K\right\rfloor-K \cdot T\right)$. The running time of the adapted dynamic program is $O\left(k \cdot T \cdot\left(R_{\max } / K\right)^{2}\right)=O\left(k \cdot T^{5} / \epsilon^{2}\right)$, since $R_{\max } \leq T \cdot R_{\min }$.

\subsubsection{A constant number of breakpoints}

For the dynamic pricing problem with additive demand change, we give in this subsection an algorithm with an exponential time complexity in the number of breakpoints. In case the number of breakpoints is constant, we therefore end up with an algorithm that runs in polynomial time.

For a given solution $\pi=\left(\pi_{0}, \ldots, \pi_{T-1}\right)$, let $\eta_{i, t}(\pi)$ indicate the number of times level price $p_{i, t}$ is chosen (and thus $\Delta_{i}$ is obtained) up to time period $t$, and let $H_{t}(\pi)=\left(\eta_{1, t}(\pi), \ldots, \eta_{k, t}(\pi)\right)$ denote a $k$-tuple of these numbers. Note that $\sum_{i=1}^{k} \eta_{i, t}(\pi)=t$. We write $H_{t}=\left(\eta_{1, t}, \ldots, \eta_{k, t}\right)$ in case $\pi$ follows from the context.

We call a tuple $H_{t+1}=\left(\eta_{1, t+1}, \ldots, \eta_{k, t+1}\right)$ reachable from tuple $H_{t}=\left(\eta_{1, t}, \ldots, \eta_{k, t}\right)$ if $H_{t+1}$ differs from $H_{t}$ in exactly one element by one. Furthermore, we say that at time period $t, C_{H_{t}}$ is a reachable customer level if there exists a tuple $H_{t}=\left(\eta_{1, t}, \ldots, \eta_{k, t}\right)$ with $\sum_{i=1}^{k} \eta_{i, t}(\pi)=t$ such that

$$
C_{H_{t}}=C_{0}+\sum_{i=k} \eta_{i, t} \cdot \Delta_{i}
$$

To obtain an optimal solution for the dynamic pricing problem, we model it as a longest path problem in a directed acyclic graph $D_{k}$. The node set $V$ is as follows. For each nonnegative customer level $C_{t}$ defined by a tuple $H_{t}$ at time period $t$, we have a node $v_{H_{t}}$. In addition, we have a sink node $v_{H_{T}}$ and we define node $v_{H_{0}}$ to be the source node. 


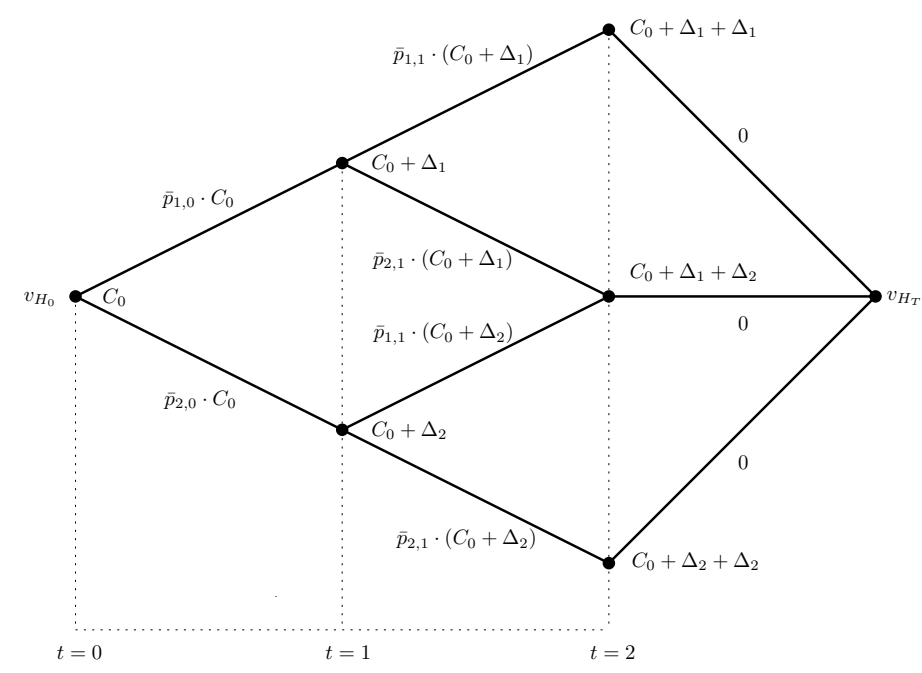

Figure 2.3: A directed acyclic graph $D_{2}$ with $T=3$

The arc set $A$ consists of $\operatorname{arcs}\left(v_{t, H_{t}}, v_{t+1, H_{t+1}}\right)$ for every $t \in\{0, \ldots, T-2\}$, where $H_{t+1}$ is reachable from $H_{t}$, and additional arcs connecting nodes $v_{H_{T-1}}$ to the sink node $v_{H_{T}}$. Each arc $\left(v_{H_{t}}, v_{H_{t+1}}\right)$ has length

$$
l\left(v_{H_{t}}, v_{H_{t+1}}\right)=p_{i, t} \cdot C_{H_{t}} \cdot \bar{F}_{t}\left(p_{i, t}\right),
$$

where $i$ denotes the index of the element in which $H_{t}$ and $H_{t+1}$ differ, and each of the arcs $\left(v_{H_{T-1}}, v_{H_{T}}\right)$ has length 0 . See Figure 2.3 for an example with $k=2$ and $T=3$.

It is easy to see that any solution with level prices to the dynamic pricing problem corresponds to a path in the graph $D_{k}$ with the same length and vice versa. We therefore complete this subsection by estimating the running time of the longest path computation in $D_{k}$.

Theorem 2.3.7. An optimal solution to the dynamic pricing problem can be found in $O\left(k \cdot T^{k}\right)$ time.

Proof. The number of distinct non-negative customer levels at time period $t$ is equal to the number of ways in which you can draw with repetition a sequence of $t$ items from a set with cardinality $k$, i.e., $\left(\begin{array}{c}k+t-1 \\ t\end{array}\right) \in O\left(t^{k-1}\right)$. By construction, we therefore have $|V| \in O\left(T^{k}\right)$ and $|A| \in O\left(k \cdot T^{k}\right)$. Next, the customer levels can be calculated in $O(k)$ time and the arc lengths in constant time. Hence, the directed acyclic graph $D_{k}$ is created in $O\left(k \cdot T^{k}\right)$ time. Further, it is well known that the longest path problem in $D_{k}$ can be solved in $O(|A|) \in O\left(k \cdot T^{k}\right)$ time. This brings us to a total time complexity of $O\left(k \cdot T^{k}\right)$.

\subsubsection{Structure of an optimal solution}

In this subsection, we investigate the structure of an optimal solution under an additive model, assuming that the reservation price distributions and breakpoints stay constant over time, i.e., $F_{t}()=.F($.$) and p_{i, t}=p_{i}$ for all $i$ and $t$. In what follows, the time indices of the prices can thus be dropped. We start by observing that the prices of an optimal solution can be arranged in a specific order. 
Lemma 2.3.8. Let $p_{j}$ be a level price. If there exists another level price, say $p_{i}$, with $p_{i}<p_{j}$ and $\bar{p}_{j} \leq \bar{p}_{i}$, then $p_{j}$ will never be chosen.

Proof. At every time period $t$, the expected revenue is given by $\pi_{t} \cdot C_{t} \cdot \bar{F}\left(\pi_{t}\right)$. Since $\bar{p}_{j} \leq \bar{p}_{i}$, choosing $\pi_{t}=p_{i}$ instead of $\pi_{t}=p_{j}$ does not decrease the expected revenue at time period $t$. In addition, we have $\Delta_{i} \geq \Delta_{j}$ and thus $\pi_{t}=p_{i}$ also results in the same or a higher customer level at time period $t+1$. Hence, $p_{j}$ will never be chosen at time period $t$.

We call the level prices that are never chosen recessive prices. Let $P^{N} \subseteq\{1, \ldots, k\}$ denote the set of indices corresponding to all non-recessive prices. If there exists a non-recessive price $p_{l}$ with $\bar{p}_{l}=0$, then $p_{l}=0$ as $\Delta_{l}=\Delta_{1} \geq \Delta_{i}$ for all $i$. This brings us to an ordering $\succ$ of the level prices based on the following definition.

Definition 2.3.9. Given $i, j \in P^{N}$, we define an order $\succ$ by:

$$
p_{i} \succ p_{j} \quad \text { if and only if } p_{i}=0 \text { or } \bar{p}_{j} \cdot \Delta_{i}>\bar{p}_{i} \cdot \Delta_{j} \text {. }
$$

The transitivity of relation $\succ$ follows directly from the definition.

In the theorem below, we show that an optimal pricing follows the above defined ordering.

Theorem 2.3.10. The level prices of an optimal solution to the dynamic pricing problem with an additive model follow the ordering $\succ$ defined on $P^{N}$.

Proof. Let $i, j \in P^{N}$ be two indices for which $p_{i} \succ p_{j}$. Further, let $\pi=\left(\pi_{0}, \ldots, \pi_{T-1}\right)$ and $\pi^{\prime}=\left(\pi_{0}^{\prime}, \ldots, \pi_{T-1}^{\prime}\right)$ denote two feasible solutions with $\pi_{t^{\prime}}=\pi_{t^{\prime}+1}^{\prime}=p_{j}, \pi_{t^{\prime}+1}=\pi_{t^{\prime}}^{\prime}=p_{i}$ for a certain time period $t^{\prime}$, and $\pi_{s}=\pi_{s}^{\prime}$ for all $s \neq t^{\prime}, t^{\prime}+1$. Note that $C_{s}(\pi)=C_{s}\left(\pi^{\prime}\right)$ for all $s \neq t^{\prime}, t^{\prime}+1$. Then,

$$
\begin{aligned}
\mathbb{E}\left[R\left(\pi^{\prime}\right)\right]-\mathbb{E}[R(\pi)] & =\sum_{t=0}^{T-1} \pi_{t}^{\prime} C_{t}(\pi) \bar{F}_{t}\left(\pi_{t}^{\prime}\right)-\sum_{t=0}^{T-1} \pi_{t} C_{t}(\pi) \bar{F}_{t}\left(\pi_{t}\right) \\
& =\bar{p}_{i}\left(C_{t^{\prime}}\left(\pi^{\prime}\right)-C_{t^{\prime}+1}(\pi)\right)+\bar{p}_{j}\left(C_{t^{\prime}+1}\left(\pi^{\prime}\right)-C_{t^{\prime}}(\pi)\right) \\
& =\bar{p}_{j} \cdot \Delta_{i}-\bar{p}_{i} \cdot \Delta_{j}>0,
\end{aligned}
$$

where the inequality follows from Definition 1 . Hence, $\mathbb{E}\left[R\left(\pi^{\prime}\right)\right]-\mathbb{E}[R(\pi)]>0$.

We remark that an optimal solution to the dynamic pricing problem can be found in $O\left(k \cdot T^{k}\right)$ time, by considering all different orderings. Further, an obvious consequence of Lemma 2.3.8 is that for every $i, j \in P^{N}$ with $i<j$ and at least one of both corresponding levels of change positive, we have that

$$
\bar{p}_{j} \cdot \Delta_{i}>\bar{p}_{i} \cdot \Delta_{j}
$$

Intuitively, Theorem 2.3.10 thus shows that over time we first choose prices such that the corresponding level of change is positive, i.e., we increase the number of customers. After this, we choose prices with smaller or even negative levels of change to increase the expected revenue. 


\subsection{Concluding remarks}

This chapter studies the computational complexity of a dynamic pricing problem for a company that sells a single product to a group of price-sensitive customers over a finite time horizon. For the problem under multiplicative demand change, we give a polynomial-time algorithm, and we show that the problem under additive demand change is NP-hard and admits an FPTAS. In our problem definition, we assume that a certain level of customer change is obtained whenever a price on a specific interval is chosen. However, our results are also true for the dynamic pricing problem with stochastic demand change. In this problem, there is a (bounded) probability distribution on the level of change of each price interval, and we replace all customer levels by expected customer levels. 


\section{Chapter 3}

\section{Scheduling Transmissions: a Game-Theoretic Approach to the ALOHA Protocol ${ }^{1}$}

\subsection{Introduction}

Wireless networks have gone through enormous changes in the recent past, developing from academic curiosities to a part of every-day life in the span of a few decades. A key characteristic of wireless networks is that nodes share the same medium (air) for their transmissions. When two or more nodes transmit simultaneously, the intended receiver receives multiple signals, and may be unable to distinguish between them. As a consequence none of the transmissions will be received correctly. This event is referred to as a collision.

In designing a wireless network, it is important to find an efficient way to schedule transmissions, as collisions have a strong negative impact on the network performance. Transmissions may be scheduled in two ways: (i) through a centralized controller that regulates the transmissions of all nodes; or (ii) by using a distributed mechanism, so that each node for itself decides when to transmit. An example of the former are cellular networks, where a base station controls the activity of the network, while the latter can be illustrated by for example wireless LAN. In this chapter, we study the distributed scenario.

For distributed networks, a so-called Medium Access Control (MAC) protocol is used to control transmissions. The earliest example of such a MAC protocol is (pure) ALOHA, introduced by Abramson [1]. If a transmission by a node using this protocol is finished and no acknowledgment is received signaling that the transmission was successful, the node waits for a random time before retransmitting. This mechanism introduces randomness into the system that helps to reduce repeated collisions.

The ALOHA protocol was subsequently refined to slotted ALOHA by Roberts [91]. This protocol introduces a number of changes compared to pure ALOHA, the most important of which is the partition of time into slots. At the beginning of a time slot, each node for itself decides whether to transmit for the duration of a single slot. By synchronizing all nodes and uniformizing the transmission duration, slotted ALOHA eliminates collisions that occur

\footnotetext{
${ }^{1}$ This chapter is based on Marbán, Van de Ven, Borm, and Hamers (2010).
} 
halfway through a transmission. Instead, collisions can occur only at the beginning of each time slot. Roberts shows that this revised protocol significantly outperforms pure ALOHA. Although many other protocols have been introduced since ALOHA, it is still popular for its simplicity, and it is still used for applications that require a simple protocol, and do not demand high performance. Examples include the transmission of control messages in satellite ground stations, see the paper of Rom and Sidi [93], and cellular networks [117].

In this chapter, we consider a wireless network where the activity of nodes is governed by the slotted ALOHA protocol. The network performance is measured by the normalized throughput, that is, the fraction of slots in which a successful transmission takes place. Generalizing the typical behavior of such networks, we allow all nodes to cooperate by adapting their transmissions to each other such that no mutual collisions occur, even if multiple nodes transmit simultaneously. However, the cooperation is such that, even when multiple nodes transmit in the same slot, only one successful transmission can be achieved. Cooperation between all nodes will increase the network performance significantly, and we are interested in how to allocate these joint throughput gains among the nodes.

To analyze this allocation problem, we consider a corresponding cooperative game, called an ALOHA game. We show that this type of games has a certain property (convexity), and we develop a set of division rules that allocate the gains from cooperation among the nodes (players). These rules are called Weighted Gain Splitting (WGS), since they take into account the size of the individual contribution of each of node to the performance gains. Furthermore, we study three solution concepts from cooperative game theory that are applied as division rules in the ALOHA game: the core [46], the Shapley value [103], and the compromise value [109]. Finally, we investigate how these rules respond to changes in the wireless network.

Previous work. Cooperative game theory applied to the field of operations research has already been studied for many decades. Consider, for example, the literature overview by Nagarajan and Sosic [82] on the issue of cooperation among supply chain partners. Another example of a paper on this subject is given by Aydinliyim and Vairaktarakis [7]. They address the possibility of cooperation among manufacturers by modeling their interactions as a cooperative sequencing game, introduced by Curiel, Pederzoli, and Tijs [31]. More examples are given by Robinson [92], Hartman and Dror [56], and Kemahlioglu-Ziya and Barthold [59]. Their papers deal with the issue of sharing the gains of inventory pooling amongst firms at different levels of the supply chain. A related problem that considers a supply chain consisting of several retailers, each facing a newsvendor problem, and warehouses is studied in the paper of Özen, Fransoo, Norde, and Slikker [83]. The retailers can increase their profits by coordinating their orders resulting in an allocation game.

There are many examples of papers outside the field of supply chain management that also apply cooperative game theory. Aloysius [4] investigates a joint venture game, in which a firm has the possibility to jointly conduct research and (new product) development with its competitors. In the paper of Bergantiños and Vidal-Puga [16], the issue of node cooperation in the minimum spanning tree problem is studied. A related paper is given by Dutta and Kar [39]. They develop a new allocation rule for minimum cost spanning tree games, and analyze the axiomatic properties of this rule. Further, Kohli and Park [61] focus on a bargaining problem in which two parties negotiate over the order quantity and average unit price. The question 
for the seller is how to determine quantity discounts for the buyer such that maximum profit is obtained. A last example is presented by Calleja, Borm, and Hendrickx [22], who introduce cooperative games for one machine job scheduling situations in which players can have more than one job to be processed and a job can be of interest to several players.

Recently, cooperative game theory has also been applied to study wireless network situations. For example, Mathur, Sankaranarayanan, and Mandayam [74, 75] study different interference channels where nodes are allowed to cooperate by jointly decoding the received signals. La and Anantharam [64] consider a setting where nodes might jam the channel for other users, coercing other nodes to give up a larger share of the channel. Moreover, Aram, Singh, Sarkar, and Kumar [5] investigate a setting where wireless service providers may share base stations and spectrum, and discuss how to design this cooperation. Lastly, Saad, Han, Debbah, Hjørungnes, and Başar [97] present an extensive overview of cooperative games and their potential applications in communication and wireless networks.

Our contribution. We consider a wireless network where the activity of nodes is governed by the slotted ALOHA protocol. Our main contribution is the investigation of the allocation of gains that arise when nodes within the network are allowed to cooperate. In Section 3.2, we present a wireless network using the slotted ALOHA protocol, and outline the nature of cooperation between nodes. Furthermore, we develop a set of division rules that allocate the gains from cooperation among the nodes. Section 3.3 discusses some background of cooperative game theory. In Section 3.4, we define and analyze the cooperative game corresponding to the wireless network situation previously introduced. In Section 3.5, we apply three solution concepts from cooperative game theory as division rules for the throughput allocation in this game: the core, the Shapley value, and the compromise value. Finally, we study in Section 3.6 how the allocations resulting from the proposed solution concepts change with the parameters of the wireless network.

\subsection{ALOHA situations}

Consider a wireless network consisting of a set of nodes $N=\{1, \ldots, n\}$ that interfere with each other. Time is divided into unit size intervals, so-called time slots. At the beginning of each time slot, node $i \in N$ transmits a packet with probability $p_{i}, 0<p_{i} \leq 1$, independent of the other nodes. We assume that the network is controllable such that each node is forced to truthfully report its transmission probability. Transmissions occupy a single slot, and may be successful depending on whether nodes transmit simultaneously or not. We are interested in the throughput $\theta_{i}$ of node $i$, defined as the fraction of slots in which node $i$ is engaged in a successful transmission. In what follows, we assume that this throughput can be expressed in an infinitely divisible transferable commodity, e.g., money.

An ALOHA situation as described above is characterized by the pair $(N, p)$, with $N$ the set of nodes, and $p=\left(p_{1}, p_{2}, \ldots, p_{n}\right)$ the vector of all transmission probabilities. We compare two types of interaction between all nodes: without cooperation and with cooperation. In the setting without cooperation, node $i$ performs a successful transmission if and only if it is the 
only node to transmit in a particular time slot. So, the throughput of node $i \in N$ is

$$
\theta_{i}=p_{i} \prod_{j \in N \backslash\{i\}}\left(1-p_{j}\right) .
$$

The aggregate throughput $\theta$ is obtained by summing over all nodes $i \in N$,

$$
\theta=\sum_{i \in N} p_{i} \prod_{j \in N \backslash\{i\}}\left(1-p_{j}\right) .
$$

Note that $0 \leq \theta \leq 1$, as in each slot at most one successful transmission takes place.

In the setting with cooperation, transmissions between all nodes in $N$ are coordinated in such a way that even when multiple nodes transmit in the same time slot no collisions occur. This can be done using clever coding schemes that can mitigate or even completely cancel the adverse effect of simultaneous transmissions on each other, cf. Li, Yeung, and Ning [67]. However, in each time slot at most a single successful transmission can take place. Under these assumptions, the aggregate throughput $\theta(N)$ is the probability that at least one node transmits, i.e.,

$$
\theta(N)=1-\prod_{i \in N}\left(1-p_{i}\right)
$$

Combining the previous two equations, we obtain the throughput gain from cooperation,

$$
\theta(N)-\theta=1-\prod_{i \in N}\left(1-p_{i}\right)-\sum_{i \in N} p_{i} \prod_{j \in N \backslash\{i\}}\left(1-p_{j}\right) .
$$

To illustrate an ALOHA situation, we discuss an example of a wireless network consisting of three nodes with different transmission probabilities.

Example 1. Consider the ALOHA situation $(N, p)$ with $N=\{1,2,3\}$ and $p=(0.4,0.3,0.5)$. Then,

$$
\begin{aligned}
\theta_{1} & =0.4(1-0.3)(1-0.5)=0.14 \\
\theta_{2} & =0.3(1-0.4)(1-0.5)=0.09 \\
\theta_{3} & =0.5(1-0.4)(1-0.3)=0.21 \\
\theta & =\sum_{i \in\{1,2,3\}} \theta_{i}=0.44 .
\end{aligned}
$$

and

$$
\theta(N)=1-(1-0.4)(1-0.3)(1-0.5)=0.79 .
$$

Hence, the throughput gain from cooperation is

$$
\theta(N)-\theta=0.79-0.44=0.35 .
$$

The throughput gain from cooperation, given in (3.2), can also be expressed in a different way. By using the identity

$$
\sum_{k=0}^{n} \sum_{\substack{D \subseteq N \\|D|=k}} \prod_{i \in D} p_{i} \prod_{i \in N \backslash D}\left(1-p_{i}\right)=1
$$


where the left-hand side covers all the combinations of nodes transmitting and non- transmitting, we find

$$
\theta(N)-\theta=\sum_{\substack{D \subseteq N \\|D| \geq 2}} \prod_{i \in D} p_{i} \prod_{i \in N \backslash D}\left(1-p_{i}\right) .
$$

Intuitively, full cooperation improves the throughput as soon as two or more nodes transmit simultaneously.

Observe that the aggregate node throughput increases under cooperation, and that node cooperation is profitable when at least two nodes are transmitting. We are interested in how to divide these gains among the nodes. Formally, a division rule for ALOHA situations is a function $r$ which assigns to every ALOHA situation $(N, p)$ a solution $r(p)=\left(r_{1}(p), \ldots, r_{n}(p)\right)$ such that

$$
r_{i}(p) \geq 0 \quad \text { (individual rationality), }
$$

for every $i \in N$, and

$$
\sum_{i \in N} r_{i}(p)=\theta(N)-\theta \quad(\text { efficiency })
$$

for any set of transmission nodes $N$.

An example of a division rule is provided by the equal division rule, which divides the throughput gains equally among all nodes in set $N$. An obvious drawback of this rule is that it does not distinguish between nodes who actually contribute to the gains and those who do not. To overcome this issue, we propose a set of division rules that take into account the contribution of different nodes to the total performance gains, as these Weighted Gain Splitting (WGS) rules divide the throughput gains from cooperation according to node activity.

Specifically, an allocation $\lambda=\left\{\lambda^{D}\right\}_{D \subseteq N}$ is a collection of $2^{|N|}$ vectors in $\mathbb{R}^{|N|}$ that each allocate the gains given a set $D \subseteq N$ of transmitting nodes. We let $\lambda^{D} \in \Lambda_{D}$, with

$$
\Lambda_{D}=\left\{\mu \in[0,1]^{|N|} \mid \forall_{i \in N \backslash D} \mu_{i}=0, \sum_{i \in N} \mu_{i}=1\right\}
$$

and we write the set of all such coalitional fraction vectors as

$$
\Lambda=\left\{\lambda=\left\{\lambda^{D}\right\}_{D \subseteq N} \mid \forall_{D \subseteq N} \lambda^{D} \in \Lambda_{D}\right\} .
$$

For any $\lambda \in \Lambda$, the corresponding weighted gain splitting rule $W G S^{\lambda}$ allocates the gains from cooperation in an ALOHA situation $(N, p)$ according to

$$
W G S_{i}^{\lambda}(p)=\sum_{\substack{D \subseteq N \\ i \in D \\|D| \geq 2}} \lambda_{i}^{D} \prod_{j \in D} p_{j} \prod_{j \in N \backslash D}\left(1-p_{j}\right),
$$

for every $i \in N$. Each WGS rule thus allocates a non-negative value to each node (individual rationality). Further, the set of all possible WGS allocations is given by

$$
W G S(p)=\left\{W G S^{\lambda}(p) \mid \lambda \in \Lambda\right\} .
$$

It is readily seen that $W G S(p)$ is convex for all possible vectors of transmission probabilities $p$.

In the following proposition, we show that WGS rules are efficient in the sense that the total amount of throughput gains is allocated to the nodes. 
Proposition 3.2.1. Let $(N, p)$ be an ALOHA situation and let $\lambda \in \Lambda$. Then,

$$
\sum_{i \in N} W G S_{i}^{\lambda}(p)=\theta(N)-\theta
$$

Proof. By (3.4) and (3.5), we get

$$
\begin{aligned}
\sum_{i \in N} W G S_{i}^{\lambda}(p) & =\sum_{i \in N} \sum_{\substack{D \subseteq N \\
i \in D \\
|D| \geq 2}} \lambda_{i}^{D} \prod_{j \in D} p_{j} \prod_{j \in N \backslash D}\left(1-p_{j}\right) \\
& =\sum_{\substack{D \subseteq N \\
|D| \geq 2}} \sum_{i \in D} \lambda_{i}^{D} \prod_{j \in D} p_{j} \prod_{j \in N \backslash D}\left(1-p_{j}\right) \\
& =\sum_{\substack{D \subseteq N \\
|D| \geq 2}} \prod_{j \in D} p_{j} \prod_{j \in N \backslash D}\left(1-p_{j}\right)=\theta(N)-\theta
\end{aligned}
$$

where the last equality follows from the definition of the throughput gain from cooperation.

To increase intuition for the WGS rules, we reconsider the ALOHA situation of Example 1 and provide a short comparison with the equal division rule.

Example 2. The total throughput gain from cooperation is $\theta(N)-\theta=0.35$. The equal division rule therefore assigns a payoff of $0.35 \cdot 1 / 3 \approx 0.1167$ to each player $i \in N=\{1,2,3\}$. In order to determine the payoffs for the WGS rule, we first need to choose an allocation. For example, $\lambda=\left\{\lambda^{D}\right\}_{D \subseteq N} \in \Lambda$ is such that

$$
\lambda_{i}^{D}= \begin{cases}\frac{1}{|D|} & \text { if } i \in D \\ 0 & \text { if } i \in N \backslash D,\end{cases}
$$

for all $D \subseteq N$. In words, a node gets for each coalition (with strictly more than one member) that it is part of, an equal proportion of the total value of this coalition. Hence, each node obtains the following amount:

$$
\begin{aligned}
& W G S_{1}^{\lambda}(p)=\frac{1}{2} \cdot[0.4 \cdot 0.3 \cdot(1-0.5)]+\frac{1}{2} \cdot[0.4 \cdot(1-0.3) \cdot 0.5]+\frac{1}{3} \cdot[0.4 \cdot 0.3 \cdot 0.5]=0.12, \\
& W G S_{2}^{\lambda}(p)=\frac{1}{2} \cdot[0.4 \cdot 0.3 \cdot(1-0.5)]+\frac{1}{2} \cdot[(1-0.4) \cdot 0.3 \cdot 0.5]+\frac{1}{3} \cdot[0.4 \cdot 0.3 \cdot 0.5]=0.095 \\
& W G S_{3}^{\lambda}(p)=\frac{1}{2} \cdot[0.4 \cdot(1-0.3) \cdot 0.5]+\frac{1}{2} \cdot[(1-0.4) \cdot 0.3 \cdot 0.5]+\frac{1}{3} \cdot[0.4 \cdot 0.3 \cdot 0.5]=0.135
\end{aligned}
$$

where $p=(0.4,0.3,0.5)$. Note that node 1 and 3 receive slightly more payoff than under the equal division rule, while node 2 receives less. That is, $W G S^{\lambda}$ with $\lambda$ as defined above benefits the nodes with the higher transmission probabilities, since in this setting these nodes add more value to the coalitional throughput gains than the node with the lowest probability. In contrast to the equal division rule, the WGS rule thus distinguishes between nodes who actually contribute to the gains and those who do not. 


\subsection{Preliminaries on cooperative games}

To analyze in more detail the possible allocations, we turn to cooperative game theory and incorporate coalitional considerations. To this end, we introduce a cooperative game corresponding to each ALOHA situation. In this section, we first give some background on cooperative game theory.

A cooperative game with transferable utility (TU-game) is characterized by a pair $(N, v)$ consisting of a non-empty, finite set of players $N$ and the characteristic function $v: 2^{N} \rightarrow \mathbb{R}$ with $v(\emptyset)=0$. Subsets of $N$ are called coalitions, and $v(S)$ represents the value of coalition $S \subseteq N$. An important property of TU-games is convexity. A game $(N, v)$ is called convex if for all $i \in N$ and $S \subseteq T \subseteq N \backslash\{i\}$,

$$
v(S \cup\{i\})-v(S) \leq v(T \cup\{i\})-v(T) .
$$

For a convex game, the marginal contribution of a player joining a coalition is thus greater than the marginal contribution for joining any subset of this coalition.

We are interested in the situation in which all players negotiate the formation of the grand coalition $N$. The question is how to divide the value $v(N)$ among the players, taking into account the values $v(S)$ of all possible coalitions $S \subseteq N$. Let $x_{i}$ represent the payoff to player $i$ and let $x \in \mathbb{R}_{+}^{N}$ with $\sum_{i \in N} x_{i}=v(N)$ denote a payoff vector. A solution concept assigns a payoff vector or a set of payoff vectors to a game. In this chapter, we are concerned with three solution concepts: the core described by Gillies [46], the Shapley value described by Shapley [103], and the compromise value described by Tijs [109].

The core of TU-game $(N, v)$, denoted by $C(v)$, is defined as the set of individual rational and efficient allocations such that it holds for every coalition that the sum of its members' payoffs is at least as large as the value of the coalition itself. Formally,

$$
C(v)=\left\{x \in \mathbb{R}^{N} \mid \sum_{i \in N} x_{i}=v(N), \forall S \subseteq N \sum_{i \in S} x_{i} \geq v(S)\right\} .
$$

Note that no coalition of players will ever leave the grand coalition $N$ when the value $v(N)$ is divided according to an element of $C(v)$. In general, the core of a game may be empty. Shapley [104], however, proved that convex games have non-empty cores.

Let $\Pi(N)$ denote the set of all permutations of $N$ and let $\sigma \in \Pi(N)$, where $\sigma(i)=k$ means that player $i$ is at the $k$-th position. We define the marginal vector $m^{\sigma}(v) \in \mathbb{R}^{N}$ with respect to $\sigma \in \Pi(N)$ as

$$
m_{i}^{\sigma}(v)=v\left(\Omega^{\sigma}(\sigma(i))\right)-v\left(\Omega^{\sigma}(\sigma(i)-1)\right),
$$

with $\Omega^{\sigma}(k)=\{j \in N \mid \sigma(j) \leq k\}$ and $m_{i}^{\sigma}$ the $i$-th component of $m^{\sigma}$ for every $i \in N$. Shapley [104] and Ichiishi [57] showed that the core of a convex game may alternatively be written as the convex hull of all marginal vectors,

$$
C(v)=\operatorname{conv}\left\{m^{\sigma}(v) \mid \sigma \in \Pi(N)\right\} .
$$

The Shapley value of TU-game $(N, v)$, denoted by $\Phi(v)$, is defined as the average of all marginal vectors $m^{\sigma}(v)$,

$$
\Phi(v)=\frac{1}{|N| !} \sum_{\sigma \in \Pi(N)} m^{\sigma}(v) .
$$


The intuition behind this definition is as follows: assume that the formation of the grand coalition takes place in a specific order $\sigma$, that is, the players join in the order $\sigma^{-1}(1), \ldots, \sigma^{-1}(n)$, and each player receives its added value to the group of players already present. The Shapley value then averages the added value (or marginal contribution) assigned to each player over all possible orders in which the grand coalition can be formed.

Let $(N, v)$ be a convex TU-game. The compromise value, denoted by $\tau(v)$, forms a compromise between the maximum and minimum payoff a player can expect to obtain in a cooperative game. For every player $i \in N$, these payoffs are given by

$$
Z_{i}(v)=v(N)-v(N \backslash\{i\}) \quad \text { and } \quad z_{i}(v)=v(\{i\}),
$$

respectively. Note that the maximum payoff $Z_{i}(v)$ is equal to the marginal contribution of player $i$ to the grand coalition $N$, while the minimum payoff $z_{i}(v)$ is just the value of a coalition consisting solely of player $i$. The compromise value is now defined by

$$
\tau(v)=\beta Z(v)+(1-\beta) z(v),
$$

where $\beta$ is the unique element of $[0,1]$ such that $\sum_{i \in N} \tau_{i}(v)=v(N)$.

\subsection{ALOHA games}

In this section, we construct a cooperative game from an ALOHA situation. The set of players is taken equal to the set of transmission nodes, and the characteristic function $v$ is defined in such a way that the value of a coalition is equal to the total throughput gain that the members of the coalition can ensure themselves by coordinating their transmissions.

Consider a coalition $S \subseteq N$ of cooperating nodes. Simultaneous transmissions by nodes in $S$ do not interfere with each other. That is, a transmission from $S$ is successful if and only if all nodes in $N \backslash S$ remain silent, and the total throughput $\theta(S)$ of the coalition $S$ is therefore given by

$$
\theta(S)=\left[1-\prod_{i \in S}\left(1-p_{i}\right)\right] \prod_{i \in N \backslash S}\left(1-p_{i}\right) .
$$

The value of coalition $S \subseteq N$ is defined as the difference between the throughput of the nodes in $S$ with and without cooperation, i.e.,

$$
v(S)=\theta(S)-\sum_{i \in S} \theta_{i}
$$

A cooperative game derived in the way described above from an $\operatorname{ALOHA}$ situation $(N, p)$ will be called an ALOHA game $(N, v)$,

Analogous to the definition of the throughput gain from cooperation, we find the following alternative expression for the coalitional gains.

Proposition 3.4.1. Let $(N, v)$ be an ALOHA game corresponding to an ALOHA situation $(N, p)$. Then,

$$
v(S)=\sum_{\substack{D \subseteq S \\|D| \geq 2}} \prod_{i \in D} p_{i} \prod_{i \in N \backslash D}\left(1-p_{i}\right),
$$

for every $S \subseteq N$. 
Proof. Let $S \subseteq N$. Plugging (3.1) and (3.10) into (3.11), we obtain

$$
\begin{aligned}
v(S) & =\left[1-\prod_{i \in S}\left(1-p_{i}\right)\right] \prod_{i \in N \backslash S}\left(1-p_{i}\right)-\sum_{i \in S} p_{i} \prod_{j \in N \backslash\{i\}}\left(1-p_{j}\right) \\
& =\prod_{i \in N \backslash S}\left(1-p_{i}\right)\left[1-\prod_{i \in S}\left(1-p_{i}\right)-\sum_{i \in S} p_{i} \prod_{j \in S \backslash\{i\}}\left(1-p_{j}\right)\right] \\
& =\prod_{i \in N \backslash S}\left(1-p_{i}\right)\left[\sum_{\substack{D \subseteq S \\
|D| \geq 2}} \prod_{i \in D} p_{i} \prod_{i \in S \backslash D}\left(1-p_{i}\right)\right] \\
& =\sum_{\substack{D \subseteq S \\
|D| \geq 2}} \prod_{i \in D} p_{i} \prod_{i \in N \backslash D}\left(1-p_{i}\right) .
\end{aligned}
$$

Note that for $S=N$, the coalitional gain $v(S)$ reduces to the aggregate throughput gain found in (3.3). When $S$ consists of a single node we see that $v(S)=0$.

To illustrate the ALOHA game $(N, v)$ constructed above, we again revisit the wireless network situation of Example 1.

Example 3. The total throughput $\theta(S)$ for all possible coalitions $S$ is:

$$
\begin{aligned}
\theta(1) & =\theta_{1}=0.14, \quad \theta(2)=\theta_{2}=0.09, \quad \theta(3)=\theta_{3}=0.21, \\
\theta(\{1,2\}) & =(1-0.5)[1-(1-0.4)(1-0.3)]=0.29, \\
\theta(\{2,3\}) & =(1-0.4)[1-(1-0.3)(1-0.5)]=0.39, \\
\theta(\{1,3\}) & =(1-0.3)[1-(1-0.4)(1-0.5)]=0.49, \\
\theta(\{1,2,3\}) & =1-(1-0.4)(1-0.3)(1-0.5)=0.79 .
\end{aligned}
$$

The ALOHA game corresponding to the ALOHA situation of Example 1 is therefore given by

\begin{tabular}{|c||c|c|c|c|c|c|c|}
\hline $\mathrm{S}$ & 1 & 2 & 3 & 12 & 23 & 13 & 123 \\
\hline $\mathrm{v}(\mathrm{S})$ & 0 & 0 & 0 & 0.06 & 0.09 & 0.14 & 0.35 \\
\hline
\end{tabular}

Consider for example, $v(\{2,3\})=0.09$, which can be determined either by subtracting 0.09 $\left(=\theta_{2}\right)$ and $0.21\left(=\theta_{3}\right)$ from $0.39(=\theta(\{2,3\}))$ or directly by Proposition 3.4.1:

$$
v(\{2,3\})=\prod_{i \in\{2,3\}} p_{i} \prod_{i \in\{1\}}\left(1-p_{i}\right)=0.3 \cdot 0.5 \cdot(1-0.4)=0.09 .
$$

We observe from Example 3 that it is more beneficial for a node to join a larger coalition than a smaller one. The marginal contribution of node 2 is, for example, 0.06 when it joins coalition $\{1\}$, while it is 0.21 when it joins coalition $\{1,3\}$. In order to prove that this holds in general, and thus that any ALOHA game is convex, we use the following auxiliary result.

Lemma 3.4.2. Let $(N, p)$ be an $A L O H A$ situation and let $(N, v)$ be the corresponding $A L O H A$ game. Further, let $T \subseteq N, i \in N \backslash T$. Then, the marginal contribution of node $i$ to $T$ is

$$
v(T \cup\{i\})-v(T)=p_{i} \sum_{\substack{D \subseteq T \\|D| \geq 1}} \prod_{j \in D} p_{j} \prod_{j \in N \backslash(D \cup\{i\})}\left(1-p_{j}\right) .
$$


Proof. By Proposition 3.4.1, we find

$$
\begin{aligned}
v(T \cup\{i\})-v(T) & =\sum_{\substack{D \subseteq T \cup\{i\} \\
|D| \geq 2}} \prod_{j \in D} p_{j} \prod_{j \in N \backslash D}\left(1-p_{j}\right)-\sum_{\substack{D \subseteq T \\
|D| \geq 2}} \prod_{j \in D} p_{j} \prod_{j \in N \backslash D}\left(1-p_{j}\right) \\
& =\sum_{\substack{D \subseteq T \cup\{i\} \\
|D| \geq 2 \\
i \in D}} \prod_{j \in D} p_{j} \prod_{j \in N \backslash D}\left(1-p_{j}\right) \\
& =p_{i} \sum_{\substack{D \subseteq T \\
|D| \geq 1}} \prod_{j \in D} p_{j} \prod_{j \in N \backslash(D \cup\{i\})}\left(1-p_{j}\right) .
\end{aligned}
$$

Note that the marginal contribution of node $i$ to $T$ can be identified as the probability that node $i$ is active, no nodes in $N \backslash(T \cup\{i\})$ are active, and at least one node in $T$ is active. Indeed, intuitively this is the only way in which coalition $T \cup\{i\}$ can improve upon $T$.

Theorem 3.4.3. Let $(N, p)$ be an ALOHA situation and let $(N, v)$ be the corresponding $A L O H A$ game. Then $(N, v)$ is convex.

Proof. Let $i \in N$ and $S \subseteq T \subseteq N \backslash\{i\}$. By Lemma 3.4.2, we have

$$
\begin{aligned}
v(T \cup\{i\})-v(T) & =p_{i} \sum_{\substack{D \subseteq T \\
|D| \geq 1}} \prod_{j \in D} p_{j} \prod_{j \in N \backslash(D \cup\{i\})}\left(1-p_{j}\right) \\
& \geq p_{i} \sum_{\substack{D \subseteq S \\
|D| \geq 1}} \prod_{j \in D} p_{j} \prod_{j \in N \backslash(D \cup\{i\})}\left(1-p_{j}\right)=v(S \cup\{i\})-v(S) .
\end{aligned}
$$

Hence, $(N, v)$ is convex.

\subsection{Solution concepts}

In this section, we use the cooperative ALOHA game introduced in Section 3.4 to study the allocation of the throughput gains. We analyze three solution concepts of the ALOHA game: the core, the Shapley value, and the compromise value.

From Theorem 3.4.3, it follows that the core can be written as the convex hull of the marginal vectors. By exploiting the specific structure of an ALOHA game, we can derive the following expression for these marginal vectors.

Lemma 3.5.1. Let $(N, p)$ be an ALOHA situation and let $(N, v)$ be the corresponding $A L O H A$ game. Further, let $\sigma \in \Pi(N)$ denote a permutation. Then, the marginal vector $m^{\sigma}(v)$ is determined by

$$
m_{i}^{\sigma}(v)=p_{i} \sum_{\substack{D \subseteq \Omega^{\sigma}(\sigma(i)-1) \\|D| \geq 1}} \prod_{j \in D} p_{j} \prod_{j \in N \backslash(D \cup\{i\})}\left(1-p_{j}\right),
$$

for every $i \in N$. 


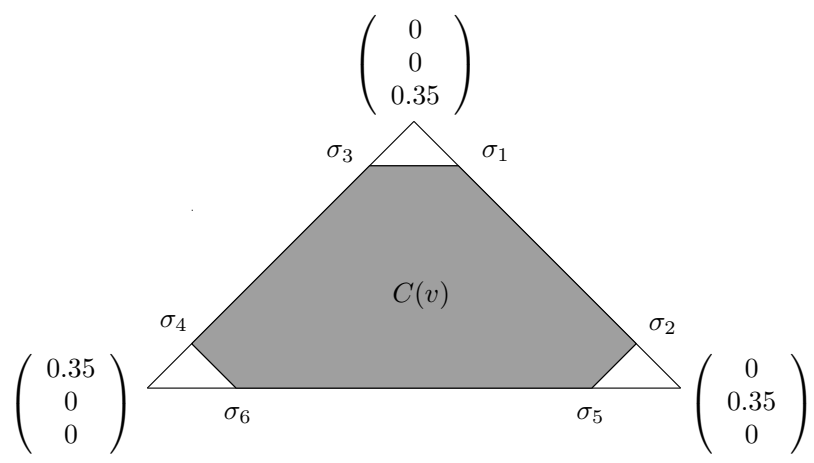

Figure 3.1: The core of the ALOHA game in Example 3.

Proof. This readily follows from applying Lemma 3.4.2 to the definition of the marginal vector (3.6), with $T=\Omega^{\sigma}(\sigma(i)-1)$.

To illustrate the connection between the core and the marginal vectors, we return to the ALOHA game of Example 3.

Example 4. The ALOHA game of Example 3 is given by

\begin{tabular}{|c||c|c|c|c|c|c|c|}
\hline$S$ & 1 & 2 & 3 & 12 & 23 & 13 & 123 \\
\hline$v(S)$ & 0 & 0 & 0 & 0.06 & 0.09 & 0.14 & 0.35 \\
\hline
\end{tabular}

and the six marginal vectors $m^{\sigma_{i}}(v)$ are

\begin{tabular}{|l|l|}
\hline$\sigma_{1}=(1,2,3)$ & $m^{\sigma_{1}}(v)=(0,0.06,0.29)$ \\
\hline$\sigma_{2}=(1,3,2)$ & $m^{\sigma_{2}}(v)=(0,0.21,0.14)$ \\
\hline$\sigma_{3}=(2,1,3)$ & $m^{\sigma_{3}}(v)=(0.06,0,0.29)$ \\
\hline$\sigma_{4}=(2,3,1)$ & $m^{\sigma_{4}}(v)=(0.26,0,0.09)$ \\
\hline$\sigma_{5}=(3,1,2)$ & $m^{\sigma_{5}}(v)=(0.14,0.21,0)$ \\
\hline$\sigma_{6}=(3,2,1)$ & $m^{\sigma_{6}}(v)=(0.26,0.09,0)$ \\
\hline
\end{tabular}

Here, $m^{\sigma_{3}}(v)$ is, for example, calculated as

$$
\left(\begin{array}{l}
m_{\sigma(1)}^{\sigma_{3}}(v) \\
m_{\sigma(2)}^{\sigma_{3}}(v) \\
m_{\sigma(3)}^{\sigma_{3}}(v)
\end{array}\right)=\left(\begin{array}{l}
m_{2}^{\sigma_{3}}(v) \\
m_{1}^{\sigma_{3}}(v) \\
m_{3}^{\sigma_{3}}(v)
\end{array}\right)=\left(\begin{array}{l}
v(2) \\
v(21)-v(2) \\
v(213)-v(21)
\end{array}\right)=\left(\begin{array}{l}
0 \\
0.06 \\
0.29
\end{array}\right) .
$$

Hence,

$$
\begin{array}{r}
C(v)=\operatorname{conv}\{(0,0.06,0.29),(0,0.21,0.14),(0.06,0,0.29), \\
(0.26,0,0.09),(0.14,0.21,0),(0.26,0.09,0)\},
\end{array}
$$

which is displayed in Figure 3.1.

Next, we analyze the relation between the core and the throughput gain allocation of the WGS rules. The following lemma states that any allocation prescribed by a WGS rule is an element of the core. 
Lemma 3.5.2. Let $(N, p)$ be an ALOHA situation and let $(N, v)$ be the corresponding ALOHA game. Then $W G S(p) \subseteq C(v)$.

Proof. Let $\lambda \in \Lambda$. By Proposition 3.2.1, we know that $W G S^{\lambda}(p)$ is efficient, and it therefore remains to show that $\sum_{i \in S} W G S_{i}^{\lambda}(p) \geq v(S)$ for any $S \subseteq N$.

By (3.4) and (3.5), we have for any $S \subseteq N$ that

$$
\begin{aligned}
\sum_{i \in S} W G S_{i}^{\lambda}(p) & =\sum_{i \in S} \sum_{\substack{D \subseteq N \\
i \in D \\
|D| \geq 2}} \lambda_{i}^{D} \prod_{j \in D} p_{j} \prod_{j \in N \backslash D}\left(1-p_{j}\right) \\
& \geq \sum_{i \in S} \sum_{\substack{D \subseteq S \\
i \in D \\
|D| \geq 2}} \lambda_{i}^{D} \prod_{j \in D} p_{j} \prod_{j \in N \backslash D}\left(1-p_{j}\right) \\
& =\sum_{\substack{D \subseteq S \\
|D| \geq 2}} \sum_{i \in D} \lambda_{i}^{D} \prod_{j \in D} p_{j} \prod_{j \in N \backslash D}\left(1-p_{j}\right) \\
& =\sum_{\substack{D \subseteq S \\
|D| \geq 2}} \prod_{j \in D} p_{j} \prod_{j \in N \backslash D}\left(1-p_{j}\right)=v(S) .
\end{aligned}
$$

Hence, $W G S^{\lambda}(p) \in C(v)$ for all $\lambda \in \Lambda$.

It turns out that the converse is also true.

Lemma 3.5.3. Let $(N, p)$ be an ALOHA situation and let $(N, v)$ be the corresponding $A L O H A$ game. Then, $C(v) \subseteq W G S(p)$.

Proof. Let $x \in C(v)$. By (3.7), we can write

$$
x=\sum_{\sigma \in \Pi(N)} \mu^{\sigma} m^{\sigma}(v)
$$

for some $\mu=\left(\mu^{\sigma}\right)_{\sigma \in \Pi(N)}$ such that $\sum_{\sigma} \mu^{\sigma}=1$ and $\mu^{\sigma} \geq 0$ for all $\sigma \in \Pi(N)$. Since $W G S(p)$ is convex for all $p$, it suffices to show for each $\sigma \in \Pi(N)$ that there exists $\lambda \in \Lambda$ such that $m^{\sigma}(v)=W G S^{\lambda}(p)$.

Let $\sigma \in \Pi(N)$. Using Lemma 3.5.1, we find

$$
\begin{aligned}
m_{i}^{\sigma}(v) & =p_{i} \sum_{\substack{D \subseteq \Omega^{\sigma}(\sigma(i)-1) \\
|D| \geq 1}} \prod_{j \in D} p_{j} \prod_{j \in N \backslash(D \cup\{i\})}\left(1-p_{j}\right) \\
& =\sum_{\substack{D \subseteq \Omega^{\sigma}(\sigma(i)) \\
i \in D \\
|D| \geq 2}} \prod_{j \in D} p_{j} \prod_{j \in N \backslash D}\left(1-p_{j}\right) \\
& =\sum_{\substack{D \subseteq N \\
i \in D \\
|D| \geq 2}} \mathrm{I}_{\left\{D \subseteq \Omega^{\sigma}(\sigma(i))\right\}} \prod_{j \in D} p_{j} \prod_{j \in N \backslash D}\left(1-p_{j}\right)=W G S_{i}^{\lambda}(p),
\end{aligned}
$$


where $\lambda=\left\{\lambda^{D}\right\}_{D \subseteq N}$ is defined as

$$
\lambda_{i}^{D}= \begin{cases}1 & \text { if } i \in D \text { and } D \subseteq \Omega^{\sigma}(\sigma(i)) \\ 0 & \text { otherwise, }\end{cases}
$$

for all $D \subseteq N$.

As a consequence of Lemma 3.5.2 and Lemma 3.5.3, the core and the set of all WGS rules coincide.

Theorem 3.5.4. Let $(N, p)$ be an $A L O H A$ situation and let $(N, v)$ be the corresponding $A L O H A$ game. Then,

$$
W G S(p)=C(v) .
$$

For convex games, a specific element of the core is the Shapley value. As ALOHA games are convex, and the set of WGS rules spans the entire core, specific elements of $\Lambda$ exist that generate the Shapley value.

Theorem 3.5.5. Let $(N, p)$ be an $A L O H A$ situation and let $(N, v)$ be the corresponding $A L O H A$ game. Choose $\lambda=\left\{\lambda^{D}\right\}_{D \subseteq N} \in \Lambda$ as

$$
\lambda_{i}^{D}= \begin{cases}\frac{1}{|D|} & \text { if } i \in D \\ 0 & \text { if } i \in N \backslash D,\end{cases}
$$

for all $D \subseteq N$. Then,

$$
\Phi(v)=W G S^{\lambda}(p) .
$$

Proof. Recall from (3.8) that for $i \in N$ we have

$$
\Phi_{i}(v)=\frac{1}{|N| !} \sum_{\sigma \in \Pi(N)} m_{i}^{\sigma}(v) .
$$

By the proof of Lemma 3.5.3, we have that

$$
\Phi_{i}(v)=\frac{1}{|N| !} \sum_{\sigma \in \Pi(N)} \sum_{\substack{D \subseteq N \\ i \in D \\|D| \geq 2}} \mathrm{I}_{\left\{D \subseteq \Omega^{\sigma}(\sigma(i))\right\}} \prod_{j \in D} p_{j} \prod_{j \in N \backslash D}\left(1-p_{j}\right) .
$$

Counting the number of permutations for which all elements of the set $D$ are positioned before node $i$, we find

$$
\begin{aligned}
\frac{1}{|N| !} \sum_{\sigma \in \Pi(N)} \mathrm{I}_{\left\{D \subseteq \Omega^{\sigma}(\sigma(i))\right\}} & =\frac{1}{|N| !} \sum_{k=|D|}^{|N|}\left(\begin{array}{c}
k-1 \\
|D|-1
\end{array}\right)(|N|-|D|) !(|D|-1) ! \\
& =\frac{1}{|D|},
\end{aligned}
$$

where the second equality follows from the combinatorial identity $\sum_{j=k}^{n}\left(\begin{array}{l}j \\ k\end{array}\right)=\left(\begin{array}{l}n+1 \\ k+1\end{array}\right)$. Substituting (3.13) into (3.12),

$$
\Phi_{i}(v)=\sum_{\substack{D \subseteq N \\ i \in D \\|D| \geq 2}} \frac{1}{|D|} \prod_{j \in D} p_{j} \prod_{j \in N \backslash D}\left(1-p_{j}\right)=W G S_{i}^{\lambda}(p) .
$$


Note that this result corresponds to the WGS rule considered in Example 2.

From Theorems 3.5.4 and 3.5.5, and the fact that WGS rules depend solely on the transmission probabilities of the nodes, it follows that both the core and the Shapley value can be obtained without any explicit knowledge of the ALOHA game. Another example of a solution concept that can be obtained independently from the game, is given in the paper of Hamers, Suijs, Tijs, and Borm [55]. In this paper, the authors consider a one-machine sequencing situation in which each agent has one job that has to be processed. They propose a solution concept that depends only on the processing times of the jobs and the weights in the cost function. This rule constitutes only a subset of the core, and explicit knowledge of the game is required to obtain the entire core.

The next theorem provides an explicit expression for the compromise value of ALOHA games. This value allocates to each node $k$ a fraction $w_{k}$ of $v(N)$. The numerator of $w_{k}$ can be interpreted as the probability that node $k$ is involved in a collision in an arbitrary time slot. So $w_{k}$ is a measure of the loss in throughput incurred from activity of node $k$. That is, it gives an indication of the gain obtained by adding node $k$ to the coalition.

Theorem 3.5.6. Let $(N, p)$ be an ALOHA situation and let $(N, v)$ be the corresponding ALOHA game. Then, $\tau_{k}(v)=w_{k} v(N)$, where

$$
w_{k}=\frac{p_{k}\left(1-\prod_{j \in N \backslash\{k\}}\left(1-p_{j}\right)\right)}{\sum_{i \in N} p_{i}\left(1-\prod_{j \in N \backslash\{i\}}\left(1-p_{j}\right)\right)}
$$

for all $k \in N$.

Proof. By Theorem 3.4.3, we know that $(N, v)$ is convex. Since $v(\{k\})=0$ for all $k \in N$, the compromise value is of the form

$$
\tau(v)=\beta Z(v),
$$

where $\beta$ is a normalizing constant. By applying the definition of $Z_{k}(v)$ from (3.9),

$$
\begin{aligned}
Z_{k}(v) & =p_{k} \sum_{\substack{D \subseteq N \backslash\{k\} \\
|D| \geq 1}} \prod_{i \in D} p_{i} \prod_{i \in N \backslash(D \cup\{k\})}\left(1-p_{i}\right) \\
& =p_{k}\left(1-\prod_{j \in N \backslash\{k\}}\left(1-p_{j}\right)\right),
\end{aligned}
$$

where the first equality follows from Lemma 3.4.2. The result then follows by substituting (3.15) into (3.14), and computing the normalization constant.

\subsection{Sensitivity analysis}

In the previous section, we presented a number of solution concepts that designate the throughput gains of cooperation among the nodes. We are interested in how sensitive these solution concepts are to changes in the ALOHA situation. Specifically, we investigate how a WGS allocation responds to changes in the transmission probabilities, and how the Shapley value is affected when the number of nodes changes. Furthermore, we consider the case where the transmission probabilities of all nodes are equal. In this case, the expression for the Shapley value simplifies and allows for a more detailed sensitivity analysis. 
Let $\lambda \in \Lambda$ be an arbitrary coalitional fraction vector and consider the corresponding WGS rule, $W G S^{\lambda}$. It is easy to see that the gains allocated to node $i \in N$ are increasing in $p_{i}$. That is,

$$
\frac{\partial}{\partial p_{i}} W G S_{i}^{\lambda}(p)=\sum_{\substack{D \subseteq N \\ i \in D \\|D| \geq 2}} \lambda_{i}^{D} \prod_{j \in D \backslash\{i\}} p_{j} \prod_{j \in N \backslash D}\left(1-p_{j}\right)>0,
$$

for every $i \in N$. Note that since the Shapley value can be characterized as a specific WGS rule, also the Shapley value for player $i$ of the corresponding ALOHA game will be increasing in $p_{i}$.

It is less straightforward in what way the WGS allocation to node $i \in N$ is affected by changes to the transmission probability of another node $k \in N \backslash\{i\}$. In fact, depending on the choice of $\lambda$, we see that $\frac{\partial}{\partial p_{k}} W G S_{i}^{\lambda}(p)$ may be either negative or positive. For example, let $i, k \in N, i \neq k$, and choose $\lambda \in \Lambda$ such that

$$
\lambda_{i}^{D}= \begin{cases}1 & i \in D \text { and } k \in D \\ 0 & \text { otherwise }\end{cases}
$$

for all $D \subseteq N$. Then,

$$
W G S_{i}^{\lambda}(p)=\sum_{\substack{D \subseteq N \\ i, k \in D \\|D| \geq 2}} \prod_{j \in D} p_{j} \prod_{j \in N \backslash D}\left(1-p_{j}\right)
$$

and

$$
\frac{\partial}{\partial p_{k}} W G S_{i}^{\lambda}(p)=\sum_{\substack{D \subseteq N \backslash\{k\} \\ i \in D \\|D| \geq 2}} \prod_{j \in D} p_{j} \prod_{j \in N \backslash D}\left(1-p_{j}\right)>0 .
$$

Conversely, if we choose $\lambda \in \Lambda$ such that

$$
\lambda_{i}^{D}= \begin{cases}1 & i \in D, \quad k \notin D \\ 0 & \text { otherwise }\end{cases}
$$

for all $D \subseteq N$, we find

$$
\frac{\partial}{\partial p_{k}} W G S_{i}^{\lambda}(p)=-\sum_{\substack{D \subseteq N \backslash\{k\} \\ i \in D \\|D| \geq 2}} \prod_{j \in D} p_{j} \prod_{j \in N \backslash(D \cup\{k\})}\left(1-p_{j}\right)<0 .
$$

Next, we investigate the impact on the Shapley value of expanding the network of ALOHA situation $(N, p)$ by a single node. To this end we introduce a second ALOHA situation $(\hat{N}, \hat{p})$ with $\hat{N}=N \cup\{t\}$ and $\hat{p}=\left(p_{1}, \ldots, p_{n}, p_{t}\right)$. We can express the Shapley value of the extended ALOHA situation in the Shapley value of the original game as follows.

Proposition 3.6.1. Let $(N, p)$ and $(\hat{N}, \hat{p})$ be two ALOHA situations described as above and let $(N, v)$ and $(\hat{N}, \hat{v})$ denote the corresponding ALOHA games. Then,

$$
\Phi_{i}(\hat{v})=p_{t} \sum_{\substack{D \subseteq N \\ i \in D \\|D| \geq 1}} \frac{1}{|D|+1} \prod_{j \in D} p_{j} \prod_{j \in N \backslash D}\left(1-p_{j}\right)+\left(1-p_{t}\right) \Phi_{i}(v)
$$

for every $i \in N$. 
Proof. Let $i \in N$. By Theorem 3.5.5, we have

$$
\begin{aligned}
\Phi_{i}(\hat{v}) & =\sum_{\substack{D \subseteq \hat{N} \\
i \in D \\
|D| \geq 2}} \frac{1}{|D|} \prod_{j \in D} p_{j} \prod_{j \in \hat{N} \backslash D}\left(1-p_{j}\right) \\
& =\sum_{\substack{D \subseteq \hat{D} \\
i \in D, t \in D \\
|D| \geq 2}} \frac{1}{|D|} \prod_{j \in D} p_{j} \prod_{j \in \hat{N} \backslash D}\left(1-p_{j}\right)+\sum_{\substack{D \subseteq \hat{N} \\
i \in D, t \in N \backslash D \\
|D| \geq 2}} \frac{1}{|D|} \prod_{j \in D} p_{j} \prod_{j \in \hat{N} \backslash D}\left(1-p_{j}\right) \\
& =p_{t} \sum_{\substack{D \subseteq N \\
i \in D}} \frac{1}{|D|+1} \prod_{j \in D} p_{j} \prod_{j \in N \backslash D}\left(1-p_{j}\right)+\left(1-p_{t}\right) \sum_{\substack{D \subseteq N \\
i \in D \\
|D| \geq 2}} \frac{1}{|D|} \prod_{j \in D} p_{j} \prod_{j \in N \backslash D}\left(1-p_{j}\right)
\end{aligned}
$$

Combining this expression with the definition of $\Phi_{i}(v)$, concludes the proof.

We can interpret $\Phi_{i}(\hat{v})$ as the Shapley value conditioned on the activity of node $t$. When node $t$ is inactive (with probability $1-p_{t}$ ) the Shapley value reduces to that of the system without node $t$ present.

The sensitivity analysis simplifies considerably when all nodes have equal transmission probabilities, i.e., $p_{i}=\rho, i \in N$. In particular, the Shapley value may be written in a more explicit form.

Proposition 3.6.2. Let $(N, p)$ be an ALOHA situation, with $p_{i}=\rho$ for all $i \in N$, and let $(N, v)$ be the corresponding ALOHA game. Then,

$$
\Phi_{i}(v)=\frac{1}{|N|}\left(1-(1-\rho)^{|N|}-|N| \rho(1-\rho)^{|N|-1}\right)
$$

for every $i \in N$.

Proof. By Theorem 3.5.5 and summing over all possible sizes of subsets $D \subseteq N$ with node $i$, we find

$$
\begin{aligned}
\Phi_{i}(v) & =\sum_{\substack{D \subseteq N \\
i \in D \\
|D| \geq 2}} \frac{1}{|D|} \rho^{|D|}(1-\rho)^{|N|-|D|}=\sum_{m=2}^{|N|} \frac{1}{m}\left(\begin{array}{c}
|N|-1 \\
m-1
\end{array}\right) \rho^{m}(1-\rho)^{|N|-m} \\
& =\frac{1}{|N|} \sum_{m=2}^{|N|}\left(\begin{array}{c}
|N| \\
m
\end{array}\right) \rho^{m}(1-\rho)^{|N|-m},
\end{aligned}
$$

for every $i \in N$. The result is then obtained by applying the binomial theorem to this expression.

The expression between brackets in (3.16) represents the fraction of time slots in which at least two nodes attempt to transmit a packet. This corresponds to the throughput gains, since these attempts would result in a collision if there is no cooperation between the nodes. The prefactor $1 /|N|$ is explained by the fact that all nodes have equal transmission probability, so the gains are spread evenly among all nodes. 
Taking the derivative of (3.16) with respect to $\rho$, we see that the Shapley value is increasing in the transmission probability,

$$
\frac{\partial}{\partial \rho} \Phi_{i}(v)=(|N|-1) \rho(1-\rho)^{|N|-2}>0,
$$

for every $i \in N$. This is explained as follows. Increasing all transmission probabilities leads to more collisions and induces higher gains from cooperation. As these gains are distributed uniformly among all nodes, the allocation by the Shapley value is increasing in $\rho$.

We finally consider the impact on the Shapley value of expanding the network by a single node, given that all nodes have equal transmission probability. In this case, the Shapley value of the extended ALOHA situation simplifies to

$$
\Phi_{i}(\hat{v})=\frac{1}{|N|+1}\left(\rho-\rho(1-\rho)^{|N|}\right)+\frac{|N|}{|N|+1}(1-\rho) \Phi_{i}(v),
$$

for every $i \in N$. It is not immediate from this expression in what way adding a node to the network affects the allocation of the Shapley value to a specific node. Indeed, depending on $|N|$ and $\rho$ the Shapley value may either increase or decrease.

Let $\Phi(n, \rho)$ denote the Shapley value of a game with $n=|N|$ nodes and equal transmission probability $\rho$ and consider $n^{*}(\rho)=\max _{n} \Phi(n, \rho)$, the number of nodes that maximizes the Shapley value given $\rho$. It is readily seen that for $\rho$ sufficiently large, $n^{*}(\rho)=2$, and that $n^{*}(\rho) \rightarrow \infty$ as $\rho \downarrow 0$. Adding a node to the network increases the gains from cooperation as the number of collisions are decreased, but also requires these gains to be shared among more nodes. Hence, for $\rho$ small enough, the increase in gains outweighs the decrease in the Shapley value due to the addition of an extra node, and for $\rho$ close to 1 , the probability of a collision is already large, and the increase in gains does not offset the additional node.

\subsection{Concluding remarks}

This chapter studies a wireless network situation in which transmissions between nodes are regulated by a slotted ALOHA protocol. When two nodes using this protocol transmit a signal at the same time slot, interference may occur resulting in lost transmissions. Cooperation between the nodes can circumvent the mutual interference, thereby having a positive impact on the network performance, measured by the total fraction of time slots in which successful transmissions take place. In order to study how the performance gains obtained from this cooperation can be allocated among all nodes in the grand coalition, we define and analyze a cooperative ALOHA game. We show that this type of game is convex and we consider three solution concepts from cooperative game theory: the core, the Shapley value, and the compromise value. Furthermore, we develop a set of Weighted Gain Splitting (WGS) allocation rules, and show that this set coincides with the core of the game. These WGS allocation rules are subsequently used to provide an alternative characterization of the Shapley value. Finally, we analyze the sensitivity of the cooperative solution concepts with respect to changes in the wireless network. 


\section{Chapter 4}

\section{The Bayesian Approach to Scheduling ${ }^{1}$}

\subsection{Introduction}

In this chapter, we consider the classical non-preemptive single machine scheduling problem to minimize the total completion time. In deterministic and traditional stochastic scheduling, this problem is well understood and can be solved to optimality by the Shortest (Expected) Processing Time (SPT or SEPT) policy: process the jobs in non-decreasing order of their (expected) processing time (Rothkopf [94] and Smith [106]). In traditional stochastic scheduling, it is assumed that the jobs' processing times are independent random variables of which the parameters, like the expected value, are fully known. We relax this assumption by introducing parameter uncertainty. Like in the papers of a.o. Burnetas and Katehakis [21], Gittins and Glazebrook [49], and Hamada and Glazebrook[53], we adopt a Bayesian viewpoint in which we have prior distributions for the uncertain parameters. These priors represent our beliefs on the values of the parameters. Furthermore, the Bayesian framework allows us to learn about the value of the parameters by processing jobs and observing their realized processing times. However, experimenting with different jobs to learn about the value of the corresponding parameters can be costly in terms of the waiting times of the still to be processed jobs. Hence, learning should be conducted carefully in order to minimize the sum of completion times in expectation.

Problem definition. There are $m$ classes of independent jobs that have to be processed by a single machine. Each class $J_{i}$ consists of $n_{i}$ jobs $(i \in\{1, \ldots, m\})$. All jobs are available for processing from the beginning and preemption of jobs is not allowed, that is, once a job has been initiated it must remain on the machine until completion. The processing time of a job in class $J_{i}$ is a random variable, which is independently and exponentially distributed with parameter $\vartheta_{i}$. Distinguishing from traditional stochastic scheduling, in the scheduling model under consideration the value of $\vartheta_{i}$ is unknown. The goal is to minimize the total completion time in expectation, $\sum_{j} \mathbb{E}\left[C_{j}\right]$.

We introduce a random variable $\Theta_{i}$ describing the scheduler's beliefs regarding the value

\footnotetext{
${ }^{1}$ This chapter is based on Marbán, Rutten, and Vredeveld (2010, 2011, 2012).
} 
of $\vartheta_{i}$. In the Bayesian approach, $\vartheta_{i}$ can be considered as a realization of the random variable $\Theta_{i}$. The initial distribution of $\Theta_{i}$, that is, before any job has been processed, is called the prior. As in Hamada and Glazebrook[53], and Hamada and Tamaki[54], we assume that the prior is a gamma distribution with parameters $\omega_{i}>0$ and $\alpha_{i}>1$. Depending on the confidence in his beliefs about $\vartheta_{i}$, the scheduler can choose the values of $\omega_{i}$ and $\alpha_{i}$ such that the prior is very peaked (the scheduler is very certain about his beliefs) or relatively flat (the scheduler is not certain about his beliefs) or anywhere in between.

After a job of class $J_{i}$ is processed, we observe this job's processing time $x$. Since the gamma distribution is a conjugate prior for the exponential distribution, the posterior distribution of $\Theta_{i}$, representing the beliefs of $\vartheta_{i}$ after having observed processing time realization $x$, is also a gamma distribution with parameters $\omega_{i}+x$ and $\alpha_{i}+1$. This result is stated in a.o. DeGroot[36] and is also derived from Bayes' theorem for probability density functions. In this way, the scheduler gradually learns about the unknown parameter, thereby enhancing his decision making in the future.

A solution to a stochastic scheduling problem is not merely a simple schedule, but a socalled scheduling policy. We follow the notion of scheduling policies as proposed by Möhring, Radermacher, and Weiss [77]. A scheduling policy makes decisions on which job to schedule at certain decision times. We require a policy to be non-anticipatory: at any time, it may not utilize the actual processing times of jobs that have not yet been completed. A scheduling policy may, of course, at any decision time, use the information that it has gathered up to this time. An optimal scheduling policy is defined as a non-anticipatory scheduling policy that minimizes the objective value in expectation. Note hereby that an optimal scheduling policy underlies the uncertainty about processing times as well as the uncertainty about the parameters.

Burnetas and Katehakis [21] and Hamada and Glazebrook [53] present optimal policies for different number of job classes. Even for the case of 2 job classes, one of which has known parameter, these policies require solving an extensive dynamic program, where in each iteration of the program a vast amount of non-linear equations needs to be solved with high accuracy. As a result, solving instances of the Bayesian scheduling problem to optimality is highly impractical, if not impossible. This is in contrast to the traditional stochastic scheduling variant of the problem in which the optimal scheduling policy is SEPT (Rothkopf [94]). The reason why SEPT is not an optimal policy in the Bayesian setting lies in the fact that when the expected processing times of the job classes are close to each other and the parameter of the class with higher expected value is more uncertain it may be beneficial to learn about the value of the underlying parameter of this class. As SEPT is a very simple policy that is optimal for the traditional stochastic scheduling problem, it is interesting to know how well it performs in the setting with parameter uncertainty.

In the Bayesian setting, there are two natural versions of SEPT. The first one, which we keep calling SEPT, determines the order in which the jobs will be processed once, at initialization and based on the original beliefs. The second version, which we denote by learning-SEPT or $\ell$-SEPT, updates its beliefs on $\vartheta_{i}$ every time a job of class $J_{i}$ is completed. After each completion of a job, $\ell$-SEPT will schedule the job with shortest expected processing time with respect to its current beliefs. In this chapter, we investigate the quality of the solution value obtained by both policies. Adopting the definition of Möhring, Schulz and Uetz [78], we define a policy $\Pi$ to be a $\rho$-approximative policy when $\mathbb{E}[\Pi(I)] \leq \rho \mathbb{E}[\mathrm{OPT}(I)]$ on any scheduling instance $I$. 
In our setting, a scheduling instance $I$ is given by the number of classes $m$ and the parameters $n_{i}, \omega_{i}$ and $\alpha_{i}$ for all classes $i \in\{1, \ldots m\}$. Further, $\mathbb{E}[\Pi(I)]$ is the expected total completion time of policy $\Pi$ on instance $I$ and OPT is the optimal non-anticipatory policy. The value $\rho$ is called the (worst case) performance guarantee of scheduling policy $\Pi$. We remark that also OPT is unaware of the values of $\vartheta_{i}$ for the various classes.

Previous work. The deterministic version of our problem can be solved to optimality by the SPT rule (Smith [106]). When preemption is allowed, i.e., jobs may be interrupted and resumed later and jobs arrive to the scheduler over time, the problem is solved by the Shortest Remaining Processing Time (SRPT) rule (Schrage [99]). On more than one machine, the performance guarantee of the SRPT rule has been studied by Phillips, Steain and Wein [86] and subsequently improved in the papers of Chung, Nonner and Souza [27] and Sitters [105].

In traditional stochastic scheduling, the processing times of jobs are random variables for which the parameters of the underlying distribution are known. Rothkopf [94] shows that WSEPT (Weighted Shortest Expected Processing Time) is an optimal policy for the stochastic single machine scheduling problem, where the objective is to minimize the sum of weighted expected completion times. Weiss [113, 114] analyzes the performance of WSEPT for the stochastic parallel machine scheduling problem. He shows asymptotic optimality of WSEPT for a certain class of processing time distributions. The first guarantee on the quality of an approximative policy was given by Möhring, Schulz, and Uetz [78]. Other approximative policies have been considered by Dean [35], Megow and Vredeveld [76], and Schulz [101]

This chapter contributes to the field by applying a Bayesian framework to the single machine scheduling problem. Examples of papers that apply the same framework to scheduling problems are limited. In the pioneering paper of Gittins and Glazebrook [49], the distributions of processing times of jobs depend all upon the same unknown parameter. The optimal schedule is obtained by calculating appropriate dynamic allocation indices, first proposed by Gittins and Jones [50]. Hamada and Glazebrook [53] present another example studying the Bayesian scheduling problem with multiple weighted job classes. The processing time distributions of these classes depend on either known or unknown parameters. Optimal policies are derived by formulating the problem as a dynamic program and using dynamic allocation indices similar to the ones in two papers of Gittins [47, 48].

Burnetas and Katehakis [21] derive dynamic programming optimality conditions for the same problem with 2 job classes: one with known and one with unknown underlying parameter. For arbitrary processing time distributions, they show that an optimal policy does not depend on the number of jobs in the class with known parameter. As a result, the optimality conditions simplify, allowing the problem to be modeled as a stopping problem. For exponential processing times, they thereafter obtain characterizations concerning the structure and properties of the optimal policy. Finally, a policy is given that approximates the decisions made by an optimal policy, in case of the number of jobs in the class with unknown parameter approaches infinity. However, no guarantees are given on the performance of the proposed policy.

There are several other papers that combine stochastic scheduling with Bayesian methodology. In [51], Glazebrook and Owen employ a comparative study in which they seek to quantify the difference between using adaptive scheduling policies based on Bayesian methodology 
and non-adaptive classical stochastic scheduling policies. The difference is called the value of an adaptive solution. Upper bounds on this value are derived for several scheduling problems on a single machine. Further, Rieder and Weishaupt [90] study a problem in which jobs may change their class with some fixed but unknown probability. A Bayesian approach is taken to update beliefs regarding these probabilities. Finally, the paper of Hamada and Tamaki [54] constitutes the first extension to two machines, for 2 job classes. Again, a dynamic programming formulation is used to derive optimality conditions.

Bayesian methodology is widely applied in research fields related to scheduling. In inventory management for example, there is a large body of literature dealing with uncertain demand distributions and Bayesian learning. Pioneered by Azoury [8] and Scarf [98], some recent papers are given by Chen and Plambeck [26], Lariviere and Porteus [65], and Lu, Song and Zhu [71]. The majority of these papers assumes that prices are exogenous and studies the problem of making optimal inventory decisions. Bayesian demand learning has also received a great deal of attention within the field of pricing, see the papers of Araman and Caldentey [6], Cope [30], and Lin [68]. All these papers are experimental in that they focus on developing heuristics and studying their computational aspects. The first, and so far only, paper to analyze the theoretical worst-case performance of a Bayesian pricing heuristic is by Farias and Van Roy [43].

Our contribution. Our main contribution is the in-depth investigation of the performance of the Shortest Expected Processing Time policy, SEPT, and an adaptive version of SEPT, $\ell$-SEPT, within a Bayesian single machine scheduling framework. We provide tight performance guarantees on the expected performance of SEPT and $\ell$-SEPT with respect to the expected performance of an optimal policy, for the following three problem settings:

- Problem Setting I: $m$ job classes with unknown parameters $\theta_{1}, \ldots, \theta_{m}$;

- Problem Setting II: 2 job classes with unknown parameters $\theta_{1}$ and $\theta_{2}$;

- Problem Setting III: 2 job classes with one known, $\mu$, and one unknown parameter, $\theta$.

In Section 4.2, we give some background on Bayesian methodology and introduce the different scheduling policies, SEPT, $\ell$-SEPT, and OPT. For Problem Setting I, we show in Section 4.3.1 that $\ell$-SEPT is in expectation better than the non-adaptive version SEPT. Furthermore, we prove that the performance guarantees for both SEPT and $\ell$-SEPT are upper bounded by $m$. In Section 4.3.2, we construct instances showing that this bound of $m$ is tight for SEPT. To our knowledge, this exemplifies one of the first tight performance guarantees in stochastic scheduling, where the tightness follows from non-degenerate processing time distributions. Moreover, we provide a lower bound of $1+\sqrt{m-1}$ on the performance guarantee of $\ell$-SEPT. In Section 4.4, we conjecture that this bound for $\ell$-SEPT is tight. For Problem Settings II and III, these results are strengthened in Sections 4.5 and 4.6. We give tight performance guarantees that depend fully on the number of jobs in both job classes, $n_{1}$ and $n_{2}$, and that are strictly less than 2 . Section 4.7 complements our theoretical findings with computational results, showing that the adaptive variant $\ell$-SEPT in practice significantly outperforms SEPT for all three problem settings. 


\subsection{Preliminaries and scheduling policies}

In this section, we introduce the Bayesian scheduling framework and policies SEPT, $\ell$-SEPT, and OPT. Additionally, we give several useful propositions.

\subsubsection{Bayesian methodology}

In traditional stochastic problems, parameters are assumed to be fixed and known. Bayesian methodology however, allows a decision maker to be uncertain about their true value. Parameters are treated as random variables, which is not a description of their variability but merely a description of the uncertainty on the side of the scheduler about their value. All available information about the true value of the parameters is combined in one expression, a probability distribution. At first it includes all existing beliefs regarding the parameters which might be present prior to seeing any data. Over time these beliefs are updated in the light of new realizations.

Formally, a random variable $\Theta_{i}$ is introduced which describes the scheduler's beliefs regarding the unknown value of parameter $\vartheta_{i}$. In the Bayesian approach, $\vartheta_{i}$ can be considered as a realization of the random variable $\Theta_{i}$. For some $\theta>0$, let $g_{i}(\theta):=\frac{d}{d \theta} \operatorname{Pr}\left[\Theta_{i} \leq \theta\right]$ denote a (prior) probability density function. Intuitively, the probability expresses how strongly we believe that the value of $\vartheta_{i}$ is less than or equal to $\theta$, prior to seeing any realization of processing times of jobs of class $J_{i}$. As mentioned before, we assume $\Theta_{i}$ follows a gamma distribution with parameters $\omega_{i}>0$ and $\alpha_{i}>1$. Let $X_{i}^{k}$ denote the random variable expressing the processing time of the $\mathrm{k}^{\text {th }}$ job of job class $J_{i}$. Once $k$ jobs of class $J_{i}$ have been completed with processing time realizations $x_{i}^{1}$ up to $x_{i}^{k}$, the beliefs with respect to the unknown value of $\vartheta_{i}$ will be updated and expressed by the (posterior) probability density function

$$
g_{i}\left(\theta \mid x_{i}^{1}, \ldots, x_{i}^{k}\right):=\frac{d}{d \theta} \operatorname{Pr}\left[\Theta_{i} \leq \theta \mid X_{i}^{1}=x_{i}^{1}, \ldots, X_{i}^{k}=x_{i}^{k}\right] .
$$

Since the gamma distribution provides a conjugate prior for the exponential distribution, the posterior is also a gamma with parameters $\omega_{i}^{\prime}:=\omega_{i}+\sum_{j=1}^{k} x_{i}^{j}$ and $\alpha_{i}^{\prime}:=\alpha_{i}+k$ (see e.g. Section 9.4 of [36]).

Updating beliefs toward $\vartheta_{i}$ results in updated beliefs regarding the processing times of uncompleted jobs in class $J_{i}$. The probability density function expressing these latter beliefs, after having completed $k$ jobs of class $J_{i}$, is denoted by

$$
f_{i}^{k}\left(x_{i}^{k+1}\right):=\frac{d}{d x_{i}^{k+1}} \operatorname{Pr}\left[X_{i}^{k+1} \leq x_{i}^{k+1} \mid X_{i}^{1}=x_{i}^{1}, \ldots, X_{i}^{k}=x_{i}^{k}\right]
$$

which is equal to

$$
\begin{aligned}
f_{i}^{k}\left(x_{i}^{k+1}\right) & =\int_{0}^{\infty} f\left(x_{i}^{k+1} \mid \theta\right) g_{i}\left(\theta \mid x_{i}^{1}, \ldots, x_{i}^{k}\right) d \theta \\
& =\int_{0}^{\infty} \theta e^{-\theta x_{i}^{k+1}} \frac{\omega_{i}^{\prime} \alpha_{i}^{\prime}}{\Gamma\left(\alpha_{i}^{\prime}\right)} \theta^{\alpha_{i}^{\prime}-1} e^{-\theta \omega_{i}^{\prime}} d \theta=\frac{\alpha_{i}^{\prime} \omega_{i}^{\prime} \alpha_{i}^{\prime}}{\left(\omega_{i}^{\prime}+x_{i}^{k+1}\right)^{\alpha_{i}^{\prime}+1}}
\end{aligned}
$$


where $f\left(x_{i}^{k+1} \mid \theta\right)$ is an exponential probability density function with parameter $\theta$. Furthermore, straightforward integration yields the first moment of $X_{i}^{k+1}$ given the first $k$ realizations:

$$
\mathbb{E}\left[X_{i}^{k+1} \mid X_{i}^{1}=x_{i}^{1}, \ldots, X_{i}^{k}=x_{i}^{k}\right]=\int_{0}^{\infty} x_{i}^{k+1} f_{i}^{k}\left(x_{i}^{k+1}\right) d x_{i}^{k+1}=\frac{\omega_{i}+\sum_{j=1}^{k} x_{i}^{j}}{\alpha_{i}+k-1} .
$$

The more jobs of job class $i$ have been processed, the more accurate the scheduler's beliefs regarding $\vartheta_{i}$ will be. First, the expected value of $\left(\Theta_{i} \mid X_{i}^{1}=x_{i}^{1}, \ldots, X_{i}^{k}=x_{i}^{k}\right)$ will converge to $\vartheta_{i}$ by the law of large numbers. Secondly, the variance of $\left(\Theta_{i} \mid X_{i}^{1}=x_{i}^{1}, \ldots, X_{i}^{k}=x_{i}^{k}\right)$ will decrease since $\omega_{i}$ and $\alpha_{i}$ will be increased with every new observation. Hence, the more jobs we process, the more peaked and the more centered around $\vartheta_{i}$ the distribution of $\left(\Theta_{i} \mid X_{i}^{1}=x_{i}^{1}, \ldots, X_{i}^{k}=\right.$ $\left.x_{i}^{k}\right)$ will become, i. e., the more we learn about the value of $\vartheta_{i}$.

\subsubsection{Bayesian scheduling policies}

An optimal policy, OPT, for the Bayesian scheduling problem at hand minimizes total completion time in expectation, thereby taking into account the uncertainty regarding the job class parameters, as well as uncertainty about the realizations. That is, the values of the parameters $\vartheta_{i}$ are unknown to OPT, but the policy will anticipate and act in its decision making upon the additional information to be revealed when processing a job of a certain class. In order to characterize OPT, we formulate the problem as a dynamic program, introduced by [53].

Let $\boldsymbol{n}=\left(n_{1}, \ldots, n_{m}\right), \boldsymbol{\omega}=\left(\omega_{1}, \ldots, \omega_{m}\right)$, and $\boldsymbol{\alpha}=\left(\alpha_{1}, \ldots, \alpha_{m}\right)$. Then, $(\boldsymbol{n}, \boldsymbol{\omega}, \boldsymbol{\alpha}) \in \mathbb{Z}_{+}^{m} \times \mathbb{R}_{>0}^{m} \times$ $\mathbb{R}_{>1}^{m}$ denotes a state vector encompassing all relevant information of the state the system is in. It consists of the remaining number of jobs in each class $J_{i}$ as well as the parameters of the current belief for $\vartheta_{i}$. Let $\boldsymbol{e}_{i}$ be the $i$ th unit vector. If in state $(\boldsymbol{n}, \boldsymbol{\omega}, \boldsymbol{\alpha})$, a job of class $J_{i}$ is processed and completed having realization $x$, then the state changes to $\left(\boldsymbol{n}-\boldsymbol{e}_{i}, \boldsymbol{\omega}+x \boldsymbol{e}_{i}, \boldsymbol{\alpha}+\boldsymbol{e}_{i}\right)$. Let $\mathbb{E}\left[\Pi^{*}(\boldsymbol{n}, \boldsymbol{\omega}, \boldsymbol{\alpha})\right]$ denote the expected sum of completion times when the optimal policy is adopted from state $(\boldsymbol{n}, \boldsymbol{\omega}, \boldsymbol{\alpha})$ onwards. Further, let $\mathbb{E}\left[\Pi_{i}^{*}(\boldsymbol{n}, \boldsymbol{\omega}, \boldsymbol{\alpha})\right]$ denote the sum of the expected completion times of a policy which first processes a job of class $J_{i}$ (assuming $n_{i} \geq 1$ ) and follows an optimal policy afterwards.

An optimal policy can then be modeled by the following dynamic program:

$$
\mathbb{E}\left[\Pi^{*}(\boldsymbol{n}, \boldsymbol{\omega}, \boldsymbol{\alpha})\right]=\min _{i \in\{1, \ldots, m\}: n_{i} \geq 1}\left\{\mathbb{E}\left[\Pi_{i}^{*}(\boldsymbol{n}, \boldsymbol{\omega}, \boldsymbol{\alpha})\right]\right\}
$$

and

$$
\mathbb{E}\left[\Pi^{*}\left(n_{i} \boldsymbol{e}_{i}, \boldsymbol{\omega}, \boldsymbol{\alpha}\right)\right]=\left(\sum_{j=1}^{n_{i}} j\right) \frac{\omega_{i}}{\alpha_{i}-1}=\frac{n_{i}\left(n_{i}+1\right)}{2} \frac{\omega_{i}}{\alpha_{i}-1} \quad \forall n_{i} \geq 0,
$$

As the length of the first job to be processed by a policy influences the completion time of all jobs, a straightforward calculation shows that

$$
\mathbb{E}\left[\Pi_{i}^{*}(\boldsymbol{n}, \boldsymbol{\omega}, \boldsymbol{\alpha})\right]=\sum_{j=1}^{m} n_{j} \frac{\omega_{i}}{\alpha_{i}-1}+\int_{0}^{\infty} \mathbb{E}\left[\Pi^{*}\left(\boldsymbol{n}-\boldsymbol{e}_{i}, \boldsymbol{\omega}+x_{i}^{1} \boldsymbol{e}_{i}, \boldsymbol{\alpha}+\boldsymbol{e}_{i}\right)\right] f_{i}^{0}\left(x_{i}^{1}\right) d x_{i}^{1},
$$


for all $i$ such that $n_{i} \geq 1$.

In the traditional stochastic scheduling variant, in which the parameters $\vartheta_{i}$ are known, the policy SEPT processes jobs in non-decreasing order of expected processing times. In the Bayesian scheduling problem at hand, SEPT processes the jobs of each job class en bloc, starting with the class having the shortest expected processing time. Formally, SEPT processes all jobs of class $J_{i}$ before any job of class $J_{k}$ if $\frac{\omega_{i}}{\alpha_{i}-1}<\frac{\omega_{k}}{\alpha_{k}-1}$. The random variable for the sum of completion times of SEPT is denoted by $\Pi^{s}$, and its expected value can be written as

$$
\mathbb{E}\left[\Pi^{s}(\boldsymbol{n}, \boldsymbol{\omega}, \boldsymbol{\alpha})\right]=\sum_{i=1}^{m} \frac{n_{i}\left(n_{i}+1\right)}{2} \frac{\omega_{i}}{\alpha_{i}-1}+\sum_{i=1}^{m-1} \sum_{j=i+1}^{m} n_{i} n_{j} \min \left\{\frac{\omega_{i}}{\alpha_{i}-1}, \frac{\omega_{j}}{\alpha_{j}-1}\right\} .
$$

for all $n \geq 0, \omega>0$ and $\alpha>1$. The non-adaptive character of SEPT could result in performance loss in comparison to a policy which makes use of additional information being revealed when processing the jobs. This shortcoming of SEPT is illustrated by the following example.

Example 1. Consider the Bayesian scheduling problem with 2 job classes and unknown parameters. Let $\omega_{1}=10, \alpha_{1}-1=90, \omega_{2}=0.2$ and $\alpha_{2}-1=2$ such that $\mathbb{E}\left[X_{1}^{1}\right]=\frac{\omega_{1}}{\alpha_{1}-1}=\frac{10}{90}>0.1$ and $\mathbb{E}\left[X_{2}^{1}\right]=\frac{\omega_{2}}{\alpha_{2}-1}=\frac{0.2}{2}=0.1$, where $X_{i}^{1}$ denotes the processing time of the first job to be processed of class $J_{i}$. Since $\mathbb{E}\left[X_{1}^{1}\right]>\mathbb{E}\left[X_{2}^{1}\right]$, SEPT will first process all jobs of class $J_{2}$ and afterward all jobs of class $J_{1}$. The values are such that the distribution of $\Theta_{1}$ is peaked, i. e., we are relatively sure about the value of $\vartheta_{1}$, whereas the distribution of $\Theta_{2}$ is flat, i. e., we are relatively unsure about the value of $\vartheta_{2}$ (see Figure 4.1). Consequently, it might be that actually $\vartheta_{2}<\vartheta_{1}$, such that, in contrast to SEPT, it would be best to first start processing all jobs of class $J_{1}$. Similar to SEPT, OPT will start processing the jobs of class $J_{2}$ since $\mathbb{E}\left[X_{2}^{1}\right]<\mathbb{E}\left[X_{1}^{1}\right]$ and the beliefs regarding $\vartheta_{2}$ are not that strong. However, in case $\vartheta_{2}<\vartheta_{1}$, OPT will observe high processing times for the first few jobs of class $J_{2}$ and realize his mistake. After processing a few jobs of class $J_{2}$, OPT will therefore switch to processing jobs of class $J_{1}$, whereas SEPT continues with processing all jobs of class $J_{2}$. By choosing appropriate values for parameters $\boldsymbol{\omega}$ and $\boldsymbol{\alpha}$, the probability that $\vartheta_{2}<\vartheta_{1}$ can be made even larger. Hence, the performance of SEPT can be far away from that of OPT.

To overcome the shortcoming discussed in the example above, we propose an adaptive policy learning-SEPT ( $\ell$-SEPT). Whenever the machine is idle, this policy starts processing the job with shortest expected processing time. Thereby, it updates the expected processing time of jobs in a class every time a job of this specific class has been completed. Formally, after $k_{i}$ jobs of class $J_{i}$ have been finished with realizations $x_{i}^{1}, \ldots, x_{i}^{k_{i}}, \ell$-SEPT starts processing a job of class $J_{h}$ in case $h=\arg \min _{i} \mathbb{E}\left[X_{i}^{k_{i}}\right]$. Note that in Example 1, both SEPT and $\ell$-SEPT start processing jobs of class $J_{2}$. However, in case $\vartheta_{2}<\vartheta_{1}$, just like OPT, $\ell$-SEPT will realize his mistake after having processed a few jobs of class $J_{2}$ and continue with processing jobs of class $J_{1}$. In what follows, $\Pi^{\ell}$ denotes the random variable for the sum of completion times when policy $\ell$-SEPT is used.

To summarize, we observe that $\ell$-SEPT uses more information than SEPT whereas OPT uses all available information, although none of the three policies know the values of $\vartheta_{i}$. All three policies know the values of $\omega_{i}$ and $\alpha_{i}$ which are derived from the scheduler's beliefs about $\vartheta_{i}$. Based on these values SEPT processes first all jobs of the job class with minimal expected 


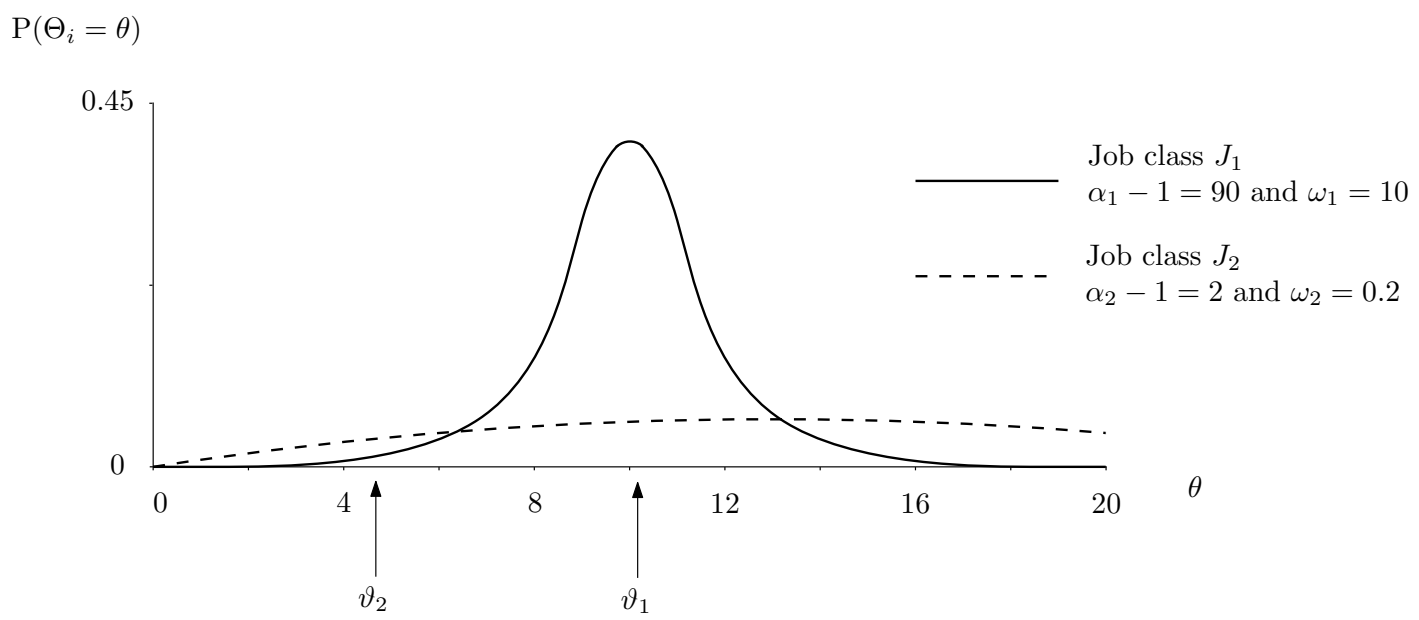

Figure 4.1: Two gamma distributions describing the beliefs with respect to the unknown parameters $\vartheta_{1}$ and $\vartheta_{2}$. The distribution corresponding to job class $J_{1}\left(J_{2}\right)$ is relatively peaked (flat) such that we are quite sure (unsure) about the value of $\vartheta_{1}\left(\vartheta_{2}\right)$.

processing time for the first job to be processed. OPT and $\ell$-SEPT are more sophisticated in the sense that they make use of the underlying distribution of $\Theta_{i}$ and update this distribution in light of new realizations. OPT in particular uses $g_{i}\left(\theta \mid x_{i}^{1}, \ldots, x_{i}^{k}\right)$ through equations (4.1), (4.3), and (4.4). $\ell$-SEPT actually only uses the first moment of the updated distribution of $\left(\Theta_{i} \mid X_{i}^{1}=x_{i}^{1}, \ldots, X_{i}^{k}=x_{i}^{k}\right)$ to determine that the expected processing time of the next job of job class $J_{i}$ equals (4.2), once $k$ jobs of job class $J_{i}$ have been processed.

In terms of decision making, one could thus interpret OPT as having a long-term view whereas SEPT and $\ell$-SEPT both have a short-term view. Both policies process a job of class $J_{i}$ only if the expected processing time of the next job in this class is minimal. OPT, however, might choose to process a job of class $J_{i}$ for which the expected processing time is not necessarily minimal. As a trade-off, OPT benefits from the additional information which is acquired regarding the uncertain parameter $\vartheta_{i}$. This information could then lead to better future decision making and a lower sum of completion times.

\subsubsection{Useful propositions}

In this subsection, we present several propositions that have an important role in the proofs of this chapter.

Proposition 4.2.1. For any $\alpha>1$,

$$
\lim _{\alpha \downarrow 1} \frac{1}{\alpha-1}\left(\frac{\alpha-1}{\alpha}\right)^{\alpha}=1
$$

Proof. Let $v(\alpha)=\alpha \ln \alpha=\ln \alpha /(1 / \alpha)$. It follows trivially from l'Hôpital's rule that

$$
\lim _{\alpha \downarrow 0} v(\alpha)=0
$$


Further, let $w(\alpha)=\frac{1}{\alpha}\left(\frac{\alpha-1}{\alpha}\right)^{\alpha-1}$. Then,

$$
\begin{aligned}
\ln w(\alpha) & =\ln \frac{1}{\alpha}\left(\frac{\alpha-1}{\alpha}\right)^{\alpha-1}=\ln \frac{(\alpha-1)^{\alpha-1}}{\alpha^{\alpha}} \\
& =(\alpha-1) \ln (\alpha-1)-\alpha \ln \alpha
\end{aligned}
$$

and

$$
\lim _{\alpha \downarrow 1} \ln w(\alpha)=\lim _{\alpha \downarrow 1}\{(\alpha-1) \ln (\alpha-1)-\alpha \ln \alpha\}=0,
$$

where the last equality follows from (4.6). Since $u=\exp (\ln u)$, this brings us to

$$
\lim _{\alpha \downarrow 1} w(\alpha)=\lim _{\alpha \downarrow 1} \exp (\ln w(\alpha))=\exp (0)=1 .
$$

Observing that

$$
\frac{1}{\alpha-1}\left(\frac{\alpha-1}{\alpha}\right)^{\alpha}=\frac{1}{\alpha}\left(\frac{\alpha-1}{\alpha}\right)^{\alpha-1}
$$

concludes the proof.

Proposition 4.2.2. For any $\omega_{i}>0$ and $\alpha_{i}>1$,

$$
\int_{0}^{\infty} \min \left\{\frac{\omega_{i}+x_{i}^{1}}{\alpha_{i}}, 1\right\} f_{i}^{0}\left(x_{i}^{1}\right) d x_{i}^{1}=\frac{\omega_{i}}{\alpha_{i}-1}-\frac{1}{\alpha_{i}-1}\left(\frac{\omega_{i}}{\alpha_{i}}\right)^{\alpha_{i}} .
$$

Proof. Let $\omega_{i}>0$ and $\alpha_{i}>1$. Then,

$$
\begin{aligned}
\int_{0}^{\infty} \min \left\{\frac{\omega_{i}+x_{i}^{1}}{\alpha_{i}}, 1\right\} f_{i}^{0}\left(x_{i}^{1}\right) d x_{i}^{1} & =\int_{0}^{\alpha_{i}-\omega_{i}} \frac{\omega_{i}+x}{\alpha_{i}} f_{i}^{0}\left(x_{i}^{1}\right) d x_{i}^{1}+\int_{\alpha_{i}-\omega_{i}}^{\infty} f_{i}^{0}\left(x_{i}^{1}\right) d x_{i}^{1} \\
& =\int_{0}^{\alpha_{i}-\omega_{i}}\left(\frac{\omega_{i}}{\omega_{i}+x_{i}^{1}}\right)^{\alpha_{i}} d x_{i}^{1}+\int_{\alpha_{i}-\omega_{i}}^{\infty} \frac{\alpha_{i} \omega_{i}^{\alpha_{i}}}{\left(\omega_{i}+x_{i}^{1}\right)^{\alpha_{1}+1}} d x_{i}^{1} \\
& =\frac{\omega_{i}}{\alpha_{i}-1}\left[1-\left(\frac{\omega_{i}}{\alpha_{i}}\right)^{\alpha_{i}-1}\right]+\left(\frac{\omega_{i}}{\alpha_{i}}\right)^{\alpha_{i}}=\frac{\omega_{i}}{\alpha_{i}-1}-\frac{1}{\alpha_{i}-1}\left(\frac{\omega_{i}}{\alpha_{i}}\right)^{\alpha_{i}} .
\end{aligned}
$$

Proposition 4.2.3. For any $\omega_{i}, \omega_{j}>0$ and $\alpha_{i}, \alpha_{j}>1$,

$$
\int_{0}^{\infty} \int_{0}^{\infty} \min \left\{\frac{\omega_{i}+x_{i}^{1}}{\alpha_{i}}, \frac{\omega_{j}+x_{j}^{1}}{\alpha_{j}}\right\} f_{j}^{0}\left(x_{j}^{1}\right) d x_{j}^{1} f_{i}^{0}\left(x_{i}^{1}\right) d x_{i}^{1}=\frac{\omega_{j}}{\alpha_{j}-1}-\frac{1}{\alpha_{j}-1}\left(\frac{\alpha_{i} \omega_{j}}{\alpha_{j} \omega_{i}}\right)^{\alpha_{j}} \frac{\omega_{i}}{\alpha_{j}+\alpha_{i}-1} .
$$

Proof. Let $\omega_{i}, \omega_{j}>0$ and $\alpha_{i}, \alpha_{j}>1$. Then,

$$
\begin{aligned}
& \int_{0}^{\infty} \int_{0}^{\infty} \min \left\{\frac{\omega_{i}+x_{i}^{1}}{\alpha_{i}}, \frac{\omega_{j}+x_{j}^{1}}{\alpha_{j}}\right\} f_{j}^{0}\left(x_{j}^{1}\right) d x_{j}^{1} f_{i}^{0}\left(x_{i}^{1}\right) d x_{i}^{1} \\
& =\int_{0}^{\infty}\left[\int_{0}^{\alpha_{j} \frac{\omega_{i}+x_{i}^{1}}{\alpha_{i}}-\omega_{j}} \frac{\omega_{j}+x_{j}^{1}}{\alpha_{j}} f_{j}^{0}\left(x_{j}^{1}\right) d x_{j}^{1}+\int_{\alpha_{j} \frac{\omega_{i}+x_{i}^{1}}{\alpha_{i}}-\omega_{j}}^{\infty} \frac{\omega_{i}+x_{i}^{1}}{\alpha_{i}} f_{j}^{0}\left(x_{j}^{1}\right) d x_{j}^{1}\right] f_{i}^{0}\left(x_{i}^{1}\right) d x_{i}^{1}
\end{aligned}
$$




$$
\begin{aligned}
& =\int_{0}^{\infty}\left[\int_{0}^{\alpha_{j} \frac{\omega_{i}+x_{i}^{1}}{\alpha_{i}}-\omega_{j}}\left(\frac{\omega_{j}}{\omega_{j}+x_{j}^{1}}\right)^{\alpha_{j}} d x_{j}^{1}+\frac{\omega_{i}+x_{i}^{1}}{\alpha_{i}} \int_{\alpha_{j} \frac{\omega_{i}+x_{i}^{1}}{\alpha_{i}}-\omega_{j}}^{\infty} \frac{\alpha_{j} \omega_{j}^{\alpha_{j}}}{\left(\omega_{j}+x_{j}^{1}\right)^{\alpha_{j}+1}} d x_{j}^{1}\right] f_{i}^{0}\left(x_{i}^{1}\right) d x_{i}^{1} \\
& =\int_{0}^{\infty}\left[\frac{\omega_{j}^{\alpha_{j}}}{1-\alpha_{j}}\left(\left[\alpha_{j} \frac{\omega_{i}+x_{i}^{1}}{\alpha_{i}}\right]^{1-\alpha_{j}}-\omega_{j}^{1-\alpha_{j}}\right)+\frac{\omega_{i}+x_{i}^{1}}{\alpha_{i}} \omega_{j}^{\alpha_{j}}\left(\alpha_{j} \frac{\omega_{i}+x_{i}^{1}}{\alpha_{i}}\right)^{-\alpha_{j}}\right] f_{i}^{0}\left(x_{i}^{1}\right) d x_{i}^{1} \\
& =\int_{0}^{\infty}\left[\frac{\omega_{j}}{\alpha_{j}-1}+\left(1-\frac{\alpha_{j}}{\alpha_{j}-1}\right) \frac{\omega_{i}+x_{i}^{1}}{\alpha_{i}}\left(\frac{\alpha_{i} \omega_{j}}{\alpha_{j}\left(\omega_{i}+x_{i}^{1}\right)}\right)^{\alpha_{j}}\right] f_{i}^{0}\left(x_{i}^{1}\right) d x_{i}^{1} \\
& =\frac{\omega_{j}}{\alpha_{j}-1}+\left(1-\frac{\alpha_{j}}{\alpha_{j}-1}\right)\left(\frac{\alpha_{i} \omega_{j}}{\alpha_{j}}\right)^{\alpha_{j}} \int_{0}^{\infty} \frac{\omega_{i}^{\alpha_{i}}}{\left(\omega_{i}+x_{i}^{1}\right)^{\alpha_{j}+\alpha_{i}}} d x_{i}^{1} \\
& =\frac{\omega_{j}}{\alpha_{j}-1}+\left(1-\frac{\alpha_{j}}{\alpha_{j}-1}\right)\left(\frac{\alpha_{i} \omega_{j}}{\alpha_{j}}\right)^{\alpha_{j}} \frac{1}{\alpha_{j}+\alpha_{i}-1} \frac{\omega_{i}^{\alpha_{i}}}{\omega_{i}^{\alpha_{j}+\alpha_{i}-1}} \\
& =\frac{\omega_{j}}{\alpha_{j}-1}-\frac{1}{\alpha_{j}-1}\left(\frac{\alpha_{i} \omega_{j}}{\alpha_{j} \omega_{i}}\right)^{\alpha_{j}} \frac{\omega_{i}}{\alpha_{j}+\alpha_{i}-1} .
\end{aligned}
$$

Proposition 4.2.4. Let $\frac{\omega_{1}}{\alpha_{1}-1}=1-\sqrt{\frac{1}{\alpha_{1}}}$. Then,

$$
\lim _{\alpha_{1} \rightarrow \infty}\left(\alpha_{1}+n_{1}-1\right)^{n_{1}-1}\left(\frac{\omega_{1}}{\alpha_{1}}\right)^{\alpha_{1}}=0 \quad \text { and } \quad \lim _{\alpha_{1} \rightarrow \infty} \frac{\omega_{1}-1}{\alpha_{1}+n_{1}-2}=\lim _{\alpha_{1} \rightarrow \infty} \frac{\omega_{1}}{\alpha_{1}-1}=1,
$$

for any $n_{1} \geq 1$.

Proof. Set $\omega_{1}=\left(\alpha_{1}-1\right)\left(1-\sqrt{\frac{1}{\alpha_{1}}}\right)<\alpha_{1}-\sqrt{\alpha_{1}}$. Then,

$$
\begin{aligned}
\lim _{\alpha_{1} \rightarrow \infty}\left(\alpha_{1}+n_{1}-1\right)^{n_{1}-1}\left(\frac{\omega_{1}}{\alpha_{1}}\right)^{\alpha_{1}} & \leq \lim _{\alpha_{1} \rightarrow \infty}\left(\alpha_{1}+n_{1}-1\right)^{n_{1}-1}\left(1-\sqrt{\frac{1}{\alpha_{1}}}\right)^{\alpha_{1}} \\
& =\lim _{\alpha_{1} \rightarrow \infty}\left(\alpha_{1}+n_{1}-1\right)^{n_{1}-1}\left[\left(\frac{\sqrt{\alpha_{1}}-1}{\sqrt{\alpha_{1}}}\right)^{\sqrt{\alpha_{1}}}\right]^{\sqrt{\alpha_{1}}} \\
& \leq \lim _{\alpha_{1} \rightarrow \infty}\left(\alpha_{1}+n_{1}-1\right)^{n_{1}-1}\left[\left(\frac{\sqrt{\alpha_{1}}+1}{\sqrt{\alpha_{1}}}\right)^{\sqrt{\alpha_{1}}}\right]^{-\sqrt{\alpha_{1}}} \\
& =\lim _{\alpha_{1} \rightarrow \infty}\left(\alpha_{1}+n_{1}-1\right)^{n_{1}-1} \cdot e^{-\sqrt{\alpha_{1}}}=0 .
\end{aligned}
$$

Further,

$$
\begin{aligned}
\lim _{\alpha_{1} \rightarrow \infty} \frac{\omega_{1}-1}{\alpha_{1}+n_{1}-2} & =\lim _{\alpha_{1} \rightarrow \infty} \frac{\left(\alpha_{1}-1\right)\left(1-\sqrt{\frac{1}{\alpha_{1}}}\right)-1}{\alpha_{1}+n_{1}-2} \\
& =\lim _{\alpha_{1} \rightarrow \infty} \frac{\left(\alpha_{1}-1\right)-1}{\alpha_{1}+n_{1}-2}=\lim _{\alpha_{1} \rightarrow \infty} \frac{\alpha_{1}-2}{\alpha_{1}+n_{1}-2}=1
\end{aligned}
$$

and

$$
\lim _{\alpha_{1} \rightarrow \infty} \frac{\omega_{1}}{\alpha_{1}-1}=\lim _{\alpha_{1} \rightarrow \infty} 1-\sqrt{\frac{1}{\alpha_{1}}}=1
$$




\subsection{Problem Setting I}

In this section, we give a tight performance guarantee of $m$ for SEPT in the problem setting with $m$ job classes. Furthermore, we give an upper bound of $m$ and a lower bound of $1+\sqrt{m-1}$ on the performance of $\ell$-SEPT with respect to OPT in the same setting.

\subsubsection{Upper bound on performance guarantees}

In this subsection, we prove that both SEPT and $\ell$-SEPT have a performance guarantee at most $m$. First, we state a trivial lower bound on the performance of an arbitrary policy. This bound is based on the fact that in any policy jobs of a class have to wait for other jobs of the same class. Hence, in constructing the lower bound we neglect waiting times caused by jobs having to wait for jobs of a different class.

Lemma 4.3.1. Let $\Pi$ be an arbitrary scheduling policy. Then, for any $m \geq 2, \boldsymbol{n}=\left(n_{1}, \ldots, n_{m}\right) \geq$ $0, \omega>0$, and $\alpha>1$,

$$
\mathbb{E}[\Pi(\boldsymbol{n}, \boldsymbol{\omega}, \boldsymbol{\alpha})] \geq \sum_{i=1}^{m} \mathbb{E}\left[\Pi\left(n_{i} \boldsymbol{e}_{i}, \boldsymbol{\omega}, \boldsymbol{\alpha}\right)\right]=\sum_{i=1}^{m} \frac{n_{i}\left(n_{i}+1\right)}{2} \frac{\omega_{i}}{\alpha_{i}-1} .
$$

Next, we show that the adaptive policy is indeed better than sequencing the jobs a priori.

Theorem 4.3.2. For any $n \geq 0, \omega>0$, and $\alpha>1$,

$$
\mathbb{E}\left[\Pi^{\ell}(\boldsymbol{n}, \boldsymbol{\omega}, \boldsymbol{\alpha})\right] \leq \mathbb{E}\left[\Pi^{s}(\boldsymbol{n}, \boldsymbol{\omega}, \boldsymbol{\alpha})\right]
$$

Proof. By (4.5), the statement is equivalent to proving that

$$
\mathbb{E}\left[\Pi^{\ell}(\boldsymbol{n}, \boldsymbol{\omega}, \boldsymbol{\alpha})\right] \leq \sum_{i=1}^{m} \frac{n_{i}\left(n_{i}+1\right)}{2} \frac{\omega_{i}}{\alpha_{i}-1}+\sum_{i=1}^{m-1} \sum_{j=i+1}^{m} n_{i} n_{j} \min \left\{\frac{\omega_{i}}{\alpha_{i}-1}, \frac{\omega_{j}}{\alpha_{j}-1}\right\},
$$

which we do by induction.

For any $n \leq 1,(4.7)$ holds with equality. Now, assume that (4.7) is true for any $\boldsymbol{n}^{\prime} \leq \boldsymbol{n}-\boldsymbol{e}_{i}$ with $i \in\{1, \ldots, m\}$, and all $\boldsymbol{\omega}>\mathbf{0}$ and $\boldsymbol{\alpha}>\mathbf{1}$. Furthermore, assume that $h=\arg \min _{i}\left\{\frac{\omega_{i}}{\alpha_{i}-1}\right\}$. 
Then, we can bound the sum of expected completion times of $\ell$-SEPT by

$$
\begin{aligned}
& \mathbb{E} {\left[\Pi^{\ell}(\boldsymbol{n}, \boldsymbol{\omega}, \boldsymbol{\alpha})\right]=\sum_{i=1}^{m} n_{i} \frac{\omega_{h}}{\alpha_{h}-1}+\int_{0}^{\infty} \mathbb{E}\left[\Pi^{\ell}\left(\boldsymbol{n}-\boldsymbol{e}_{h}, \boldsymbol{\omega}+x_{h}^{1} \boldsymbol{e}_{h}, \boldsymbol{\alpha}+\boldsymbol{e}_{h}\right)\right] f_{h}^{0}\left(x_{h}^{1}\right) d x_{h}^{1} } \\
& \stackrel{\text { (induction) }}{\leq} \sum_{i=1}^{m} n_{i} \frac{\omega_{h}}{\alpha_{h}-1}+\int_{0}^{\infty}\left(\sum_{i \neq h} \frac{n_{i}\left(n_{i}+1\right)}{2} \frac{\omega_{i}}{\alpha_{i}-1}+\frac{\left(n_{h}-1\right) n_{h}}{2} \frac{\omega_{h}+x_{h}^{1}}{\alpha_{h}}\right. \\
&\left.\quad+\sum_{i \neq h} \sum_{j \geq i+1, j \neq h} n_{i} n_{j} \min \left\{\frac{\omega_{i}}{\alpha_{i}-1}, \frac{\omega_{j}}{\alpha_{j}-1}\right\}+\sum_{i \neq h} n_{i}\left(n_{h}-1\right) \min \left\{\frac{\omega_{i}}{\alpha_{i}-1}, \frac{\omega_{h}+x_{h}^{1}}{\alpha_{h}}\right\}\right) f_{h}^{0}\left(x_{h}^{1}\right) d x_{h}^{1} \\
& \leq \sum_{i \neq h} n_{i} \frac{\omega_{h}}{\alpha_{h}-1}+\sum_{i=1}^{m} \frac{n_{i}\left(n_{i}+1\right)}{2} \frac{\omega_{i}}{\alpha_{i}-1}+\sum_{i \neq h} \sum_{j \geq i+1, j \neq h} n_{i} n_{j} \min \left\{\frac{\omega_{i}}{\alpha_{i}-1}, \frac{\omega_{j}}{\alpha_{j}-1}\right\} \\
& \quad+\sum_{i \neq h} n_{i}\left(n_{h}-1\right) \frac{\omega_{h}}{\alpha_{h}-1} \\
&=\sum_{i=1}^{m} \frac{n_{i}\left(n_{i}+1\right)}{2} \frac{\omega_{i}}{\alpha_{i}-1}+\sum_{i=1}^{m-1} \sum_{j=i+1}^{m} n_{i} n_{j} \min \left\{\frac{\omega_{i}}{\alpha_{i}-1}, \frac{\omega_{j}}{\alpha_{j}-1}\right\}
\end{aligned}
$$

where the last equality is due to the fact that $\frac{\omega_{h}}{\alpha_{h}-1}=\min _{i}\left\{\frac{\omega_{i}}{\alpha_{i}-1}\right\}$, by definition of $h$.

Given the relation between SEPT and $\ell$-SEPT, we can prove the performance guarantee on both SEPT and $\ell$-SEPT.

Theorem 4.3.3. For any $n \geq 0, \omega>0$, and $\alpha>1$,

$$
\frac{\mathbb{E}\left[\Pi^{\ell}(\boldsymbol{n}, \boldsymbol{\omega}, \boldsymbol{\alpha})\right]}{\mathbb{E}\left[\Pi^{*}(\boldsymbol{n}, \boldsymbol{\omega}, \boldsymbol{\alpha})\right]} \leq \frac{\mathbb{E}\left[\Pi^{s}(\boldsymbol{n}, \boldsymbol{\omega}, \boldsymbol{\alpha})\right]}{\mathbb{E}\left[\Pi^{*}(\boldsymbol{n}, \boldsymbol{\omega}, \boldsymbol{\alpha})\right]} \leq m
$$

Proof. The first inequality follows directly from Theorem 4.3.2. To prove the second inequality, we assume without loss of generality

$$
\frac{\omega_{1}}{\alpha_{1}-1} \leq \frac{\omega_{2}}{\alpha_{2}-1} \leq \ldots \leq \frac{\omega_{m}}{\alpha_{m}-1}
$$


Then,

$$
\begin{aligned}
0 & \leq \sum_{i=1}^{m-1} \sum_{j=i+1}^{m}\left(n_{i}-n_{j}\right)^{2} \frac{\omega_{i}}{\alpha_{i}-1}+(m-1) \sum_{i=1}^{m} n_{i} \frac{\omega_{i}}{\alpha_{i}-1} \\
& \stackrel{(4.8)}{\leq} \sum_{i=1}^{m-1} \sum_{j=i+1}^{m}\left(n_{i}^{2} \frac{\omega_{i}}{\alpha_{i}-1}+n_{j}^{2} \frac{\omega_{j}}{\alpha_{j}-1}-2 n_{i} n_{j} \frac{\omega_{i}}{\alpha_{i}-1}\right)+(m-1) \sum_{i=1}^{m} n_{i} \frac{\omega_{i}}{\alpha_{i}-1} \\
& =\sum_{i=1}^{m-1} \sum_{j=i+1}^{m} n_{i}^{2} \frac{\omega_{i}}{\alpha_{i}-1}+\sum_{j=2}^{m} \sum_{i=1}^{j-1} n_{j}^{2} \frac{\omega_{j}}{\alpha_{j}-1}-2 \sum_{i=1}^{m-1} \sum_{j=i+1}^{m} n_{i} n_{j} \frac{\omega_{i}}{\alpha_{i}-1}+(m-1) \sum_{i=1}^{m} n_{i} \frac{\omega_{i}}{\alpha_{i}-1} \\
& =(m-1) \sum_{i=1}^{m}\left(n_{i}^{2}+n_{i}\right) \frac{\omega_{i}}{\alpha_{i}-1}-2 \sum_{i=1}^{m-1} \sum_{j=i+1}^{m} n_{i} n_{j} \min \left\{\frac{\omega_{i}}{\alpha_{i}-1}, \frac{\omega_{j}}{\alpha_{j}-1}\right\} \\
& =2 m \sum_{i=1}^{m} \frac{n_{i}\left(n_{i}+1\right)}{2} \frac{\omega_{i}}{\alpha_{i}-1}-2\left(\sum_{i=1}^{m} \frac{n_{i}\left(n_{i}+1\right)}{2} \frac{\omega_{i}}{\alpha_{i}-1}+\sum_{i=1}^{m-1} \sum_{j=i+1}^{m} n_{i} n_{j} \min \left\{\frac{\omega_{i}}{\alpha_{i}-1}, \frac{\omega_{j}}{\alpha_{j}-1}\right\}\right) \\
& \leq 2\left(m \mathbb{E}\left[\Pi^{*}(\boldsymbol{n}, \boldsymbol{\omega}, \boldsymbol{\alpha})\right]-\mathbb{E}\left[\Pi^{s}(\boldsymbol{n}, \boldsymbol{\omega}, \boldsymbol{\alpha})\right]\right),
\end{aligned}
$$

where the last inequality is due to Lemma 4.3.1 and expression (4.5). Hence, we have that $\mathbb{E}\left[\Pi^{s}(\boldsymbol{n}, \boldsymbol{\omega}, \boldsymbol{\alpha})\right] \leq m \mathbb{E}\left[\Pi^{*}(\boldsymbol{n}, \boldsymbol{\omega}, \boldsymbol{\alpha})\right]$.

\subsubsection{Lower bounds on performance guarantees}

In this section, we show by an intuitive argument that the performance guarantee shown in the previous section is tight for SEPT. Further, we give a rather technical lower bound on the performance guarantee of $\ell$-SEPT, which is much lower than the upper bound stated in Theorem 4.3.3.

Matching lower bound for SEPT. We show that for any $\epsilon>0$ there exists an instance for which the ratio of the value of SEPT to the value of OPT is only an additive $\epsilon$ away from the performance guarantee of Theorem 4.3.3. Intuitively, we set our parameters in the instance in such a way that $\mathbb{E}\left[X_{1}^{1}\right]$ is slightly less than $\mathbb{E}\left[X_{i}^{1}\right]$ for all $i \in\{2, \ldots, m\}$. Then, SEPT starts processing all jobs of class $J_{1}$, followed by the jobs of the other classes. However, in case we also set the parameters such that the distributions of the $\Theta_{i}$ 's with $i \in\{2, \ldots, m\}$ are relatively flat, OPT starts processing jobs of the corresponding other classes: it is beneficial for OPT to process a few jobs of these classes to get a better idea about the value of $\vartheta_{i}(i \in\{2, \ldots, m\})$. This brings us to the following lower bound on SEPT and an upper bound on OPT.

Lemma 4.3.4. For any $n \geq 0$, there exist parameter settings $\boldsymbol{\omega}>\mathbf{0}$, and $\boldsymbol{\alpha}>\mathbf{1}$ such that

$$
\frac{\omega_{1}}{\alpha_{1}-1}<\frac{\omega_{2}}{\alpha_{2}-1}=\ldots=\frac{\omega_{m}}{\alpha_{m}-1}=1
$$

and

$$
\mathbb{E}\left[\Pi^{s}(\boldsymbol{n}, \boldsymbol{\omega}, \boldsymbol{\alpha})\right]>\sum_{i=1}^{m} \frac{n_{i}\left(n_{i}+1\right)}{2}+\sum_{i=1}^{m-1} \sum_{j=i+1}^{m} n_{i} n_{j}-\epsilon,
$$

for any $\epsilon>0$. 
Proof. Let $\epsilon^{\prime}>0$. For any $\alpha_{1}>1$ and $\alpha_{j}>1(j \in\{2, \ldots, m\})$, let $\omega_{1}=\left(1-\epsilon^{\prime}\right)\left(\alpha_{1}-1\right)$ and $\omega_{j}=\alpha_{j}-1$. By (4.5), we have

$$
\mathbb{E}\left[\Pi^{s}(\boldsymbol{n}, \boldsymbol{\omega}, \boldsymbol{\alpha})\right]=\frac{n_{1}\left(n_{1}+1\right)}{2}\left(1-\epsilon^{\prime}\right)+\sum_{i=2}^{m} \frac{n_{i}\left(n_{i}+1\right)}{2}+\sum_{j=2}^{m} n_{1} n_{j}\left(1-\epsilon^{\prime}\right)+\sum_{i=2}^{m-1} \sum_{j=i+1}^{m} n_{i} n_{j},
$$

for any $\boldsymbol{n}>\mathbf{0}$. Hence, for any $\epsilon>0$, there exists an $\epsilon^{\prime}>0$ for which the lemma holds.

Lemma 4.3.5. For any $\boldsymbol{n} \geq 0$, there exist parameter settings $\boldsymbol{\omega}>\mathbf{0}$, and $\boldsymbol{\alpha}>\mathbf{1}$ such that

$$
\frac{\omega_{1}}{\alpha_{1}-1}<\frac{\omega_{2}}{\alpha_{2}-1}=\ldots=\frac{\omega_{m}}{\alpha_{m}-1}=1
$$

and

$$
\mathbb{E}\left[\Pi^{*}(\boldsymbol{n}, \boldsymbol{\omega}, \boldsymbol{\alpha})\right]<m \sum_{i=1}^{m} n_{i}+\sum_{i=1}^{m} \frac{n_{i}\left(n_{i}+1\right)}{2}+\epsilon,
$$

for any $\epsilon>0$.

Proof. Consider the following policy П: first process subsequently one job of class $J_{2}$ up to class $J_{m}$, observing realizations $x_{2}^{1}, \ldots, x_{m}^{1}$, and schedule all remaining jobs according to SEPT. Note that the processing time distributions of the remaining jobs are updated in light of the observed realizations such that SEPT schedules the jobs based on the updated expected processing times. Then,

$$
\begin{array}{rl}
\mathbb{E}\left[\Pi^{*}(\boldsymbol{n}, \boldsymbol{\omega}, \boldsymbol{\alpha})\right] \leq & \mathbb{E}[\Pi(\boldsymbol{n}, \boldsymbol{\omega}, \boldsymbol{\alpha})]=\sum_{i=1}^{m} n_{i} \sum_{j=2}^{m} \frac{\omega_{j}}{\alpha_{j}-1}-\sum_{h=3}^{m}(h-2) \frac{\omega_{h}}{\alpha_{h}-1} \\
& +\int_{0}^{\infty} \cdots \int_{0}^{\infty} \mathbb{E}\left[\Pi^{s}\left(\boldsymbol{n}-\sum_{i=2}^{m} \boldsymbol{e}_{i}, \boldsymbol{\omega}+\sum_{i=2}^{m} x_{i}^{1} \boldsymbol{e}_{i}, \boldsymbol{\alpha}+\sum_{i=2}^{m} \boldsymbol{e}_{i}\right)\right] f_{2}^{0}\left(x_{2}^{1}\right) d x_{2}^{1} \cdots f_{m}^{0}\left(x_{m}^{1}\right) d x_{m}^{1} \\
\stackrel{(4.5)}{<} & m \sum_{i=1}^{m} n_{i}+\frac{n_{1}\left(n_{1}+1\right)}{2} \frac{\omega_{1}}{\alpha_{1}-1}+\sum_{i=2}^{m} \frac{\left(n_{i}-1\right) n_{i}}{2} \frac{\omega_{i}}{\alpha_{i}-1} \\
& +\sum_{i=2}^{m} n_{1}\left(n_{i}-1\right) \int_{0}^{\infty} \min \left\{\frac{\omega_{1}}{\alpha_{1}-1}, \frac{\omega_{i}+x_{i}^{1}}{\alpha_{i}}\right\} f_{i}^{0}\left(x_{i}^{1}\right) d x_{i}^{1} \\
& +\sum_{i=2}^{m-1} \sum_{j=i+1}^{m}\left(n_{i}-1\right)\left(n_{j}-1\right) \int_{0}^{\infty} \int_{0}^{\infty} \min \left\{\frac{\omega_{i}+x_{i}^{1}}{\alpha_{i}}, \frac{\omega_{j}+x_{j}^{1}}{\alpha_{j}}\right\} f_{i}^{0}\left(x_{i}^{1}\right) d x_{i}^{1} f_{j}^{0}\left(x_{j}^{1}\right) d x_{j}^{1} \\
\text { Prop. } 4.2 .3 & m \sum_{i=1}^{m} n_{i}+\frac{n_{1}\left(n_{1}+1\right)}{2}+\sum_{i=2}^{m} \frac{\left(n_{i}-1\right) n_{i}}{2}
\end{array}
$$$$
+\sum_{i=2}^{m} n_{1}\left(n_{i}-1\right) \int_{0}^{\infty} \min \left\{1, \frac{\omega_{i}+x_{i}^{1}}{\alpha_{i}}\right\} f_{i}^{0}\left(x_{i}^{1}\right) d x_{i}^{1}
$$$$
+\sum_{i=2}^{m-1} \sum_{j=i+1}^{m}\left(n_{i}-1\right)\left(n_{j}-1\right)\left(\frac{\omega_{j}}{\alpha_{j}-1}-\frac{1}{\alpha_{j}-1}\left(\frac{\alpha_{i} \omega_{j}}{\alpha_{j} \omega_{i}}\right)^{\alpha_{j}} \frac{\omega_{i}}{\alpha_{j}+\alpha_{i}-1}\right)
$$ 


$$
\begin{aligned}
\text { Prop. } & \text { 4.2.2 } \\
& +\sum_{i=2}^{m} n_{i=1}^{m} n_{i}+\frac{n_{1}\left(n_{1}+1\right)}{2}+\sum_{i=2}^{m} \frac{\left(n_{i}-1\right) n_{i}}{2} \frac{\omega_{i}}{\alpha_{i}-1} \\
& +\sum_{i=2}^{m-1} \sum_{j=i+1}^{m}\left(n_{i}-1\right)\left(n_{j}-1\right)\left(\frac{1}{\alpha_{i}-1}\left(\frac{\omega_{i}}{\alpha_{i}}\right)^{\alpha_{i}}\right)
\end{aligned}
$$

where in the third inequality we further use the fact that $\omega_{1}<\alpha_{1}-1$. Next, we set $\omega_{2}=\ldots=$ $\omega_{m}=\alpha-1$ and $\alpha_{2}=\ldots=\alpha_{m}=\alpha$. Then,

$$
\begin{aligned}
\mathbb{E}\left[\Pi^{*}(\boldsymbol{n}, \boldsymbol{\omega}, \boldsymbol{\alpha})\right]< & m \sum_{i=1}^{m} n_{i}+\frac{n_{1}\left(n_{1}+1\right)}{2}+\sum_{i=2}^{m} \frac{\left(n_{i}-1\right) n_{i}}{2} \\
& +\sum_{i=2}^{m} n_{1}\left(n_{i}-1\right)\left(1-\frac{1}{\alpha-1}\left(\frac{\alpha-1}{\alpha}\right)^{\alpha}\right) \\
& +\sum_{i=2}^{m-1} \sum_{j=i+1}^{m}\left(n_{i}-1\right)\left(n_{j}-1\right)\left(1-\frac{1}{2 \alpha-1}\right) .
\end{aligned}
$$

Combining (4.9) and Proposition 4.2.1, and letting $\alpha$ tend to 1 from above, we find

$$
\lim _{\alpha \downarrow 1} \mathbb{E}\left[\Pi^{*}(\boldsymbol{n}, \boldsymbol{\omega}, \boldsymbol{\alpha})\right] \leq m \sum_{i=1}^{m} n_{i}+\frac{n_{1}\left(n_{1}+1\right)}{2}+\sum_{i=2}^{m} \frac{\left(n_{i}-1\right) n_{i}}{2} .
$$

Hence, it follows that for any $\boldsymbol{n}>\mathbf{0}, \omega_{1}<\alpha_{1}-1$, there exists for any $\epsilon>0$ an $\alpha^{*}>1$ such that for all $1<\alpha_{2}=\ldots=\alpha_{m}=\omega_{2}+1=\ldots=\omega_{m}+1<\alpha^{*}$

$$
\mathbb{E}\left[\Pi^{*}(\boldsymbol{n}, \boldsymbol{\omega}, \boldsymbol{\alpha})\right]<m \sum_{i=1}^{m} n_{i}+\sum_{i=1}^{m} \frac{n_{i}\left(n_{i}+1\right)}{2}+\epsilon .
$$

Combining Lemmata 4.3.4 and 4.3.5 and letting the number of jobs in all classes approach infinity, we obtain the following theorem.

Theorem 4.3.6. There exist parameter settings $\boldsymbol{n}>\mathbf{0}, \boldsymbol{\omega}>\mathbf{0}$ and $\boldsymbol{\alpha}>\mathbf{1}$, such that

$$
\frac{\mathbb{E}\left[\Pi^{s}(\boldsymbol{n}, \boldsymbol{\omega}, \boldsymbol{\alpha})\right]}{\mathbb{E}\left[\Pi^{*}(\boldsymbol{n}, \boldsymbol{\omega}, \boldsymbol{\alpha})\right]}>m-\epsilon,
$$

for any $\epsilon>0$.

Proof. The restrictions imposed on $\omega$ and $\alpha$ in Lemmas 4.3 .4 and 4.3.5 can be satisfied simultaneously. In addition, set $n_{1}=\ldots=n_{m}=n$ and let $n$ tend to infinity. Then,

$$
\begin{aligned}
\lim _{n \rightarrow \infty} \frac{\mathbb{E}\left[\Pi^{s}(n, \ldots, n, \boldsymbol{\omega}, \boldsymbol{\alpha})\right]}{\mathbb{E}\left[\Pi^{*}(n, \ldots, n, \boldsymbol{\omega}, \boldsymbol{\alpha})\right]} & >\lim _{n \rightarrow \infty} \frac{m n^{2}+m n+m(m-1) n^{2}}{2 m^{2} n+m n^{2}+m n}-\epsilon \\
& =\lim _{n \rightarrow \infty} \frac{m n+1}{n+1+2 m}-\epsilon=m-\epsilon .
\end{aligned}
$$


Lower bound on the performance guarantee of $\ell$-SEPT. We show that the performance guarantee of $\ell$-SEPT is no less than $1+\sqrt{m-1}$. In order to obtain this result, we create a bad instance for the performance of $\ell$-SEPT, which is based on the structure of the worst case instance of SEPT, given in the proofs of Lemmas 4.3.4 and 4.3.5. To find this instance, we need to make sure that $\ell$-SEPT does not switch to processing jobs from the second class after it processed a few jobs of the first class. This is done by setting the values of $\omega_{1}$ and $\alpha_{1}$ extremely large such that we are very sure about the value of $\vartheta_{1}$. Consequently, the realizations of processing times of jobs from class $J_{1}$ barely affect the expected processing time for the next job to be processed, i. e., when $\omega_{1}$ and $\alpha_{1}$ are big enough we have

$$
\frac{\omega_{1}+\sum_{j=1}^{k} x_{1}^{j}}{\alpha_{1}+k-1} \approx \frac{\omega_{1}}{\alpha_{1}-1}=1-\epsilon<1=\frac{\omega_{2}}{\alpha_{2}-1}=\ldots=\frac{\omega_{m}}{\alpha_{m}-1}
$$

after $k$ observations on the first job class. In addition, we again need the number of jobs in all classes to tend to infinity. This gives the following theorem.

Theorem 4.3.7. There exist parameter settings $n \geq 0, \omega>0, \alpha>1$ such that

$$
\frac{\mathbb{E}\left[\Pi^{\ell}(\boldsymbol{n}, \boldsymbol{\omega}, \boldsymbol{\alpha})\right]}{\mathbb{E}\left[\Pi^{*}(\boldsymbol{n}, \boldsymbol{\omega}, \boldsymbol{\alpha})\right]}>1+\sqrt{m-1}-\epsilon
$$

for any $\epsilon>0$.

A formal proof of this theorem can be found in Appendix 4.9.1.

\subsection{Tighter performance bound for $\ell$-SEPT}

In this section, we conjecture that the performance guarantee of $\ell$-SEPT with respect to OPT is upper bounded by $1+\sqrt{m-1}$, thereby closing the gap between upper and lower bound of Theorems 4.3.3 and 4.3.7. In order to motivate this conjecture, we first propose a lower bound on the performance of an arbitrary policy and an upper bound on the performance of $\ell$-SEPT in a Bayesian scheduling framework.

As mentioned before, SEPT is an optimal policy for the scheduling problem in which all parameter values are known (Rothkopf [94]). The minimum total completion time in expectation under complete information for parameters $\theta_{1}, \ldots, \theta_{m}$, is thus given by

$$
\sum_{i=1}^{m} \frac{n_{i}\left(n_{i}+1\right)}{2} \frac{1}{\theta_{i}}+\sum_{i=1}^{m-1} \sum_{j=i+1}^{m} n_{i} n_{j} \min \left\{\frac{1}{\theta_{i}}, \frac{1}{\theta_{j}}\right\} .
$$

In case each parameter is a random variable $\Theta_{i}$ instead, the expected value of (4.10) with respect to the prior parameter distribution $g_{i}(\theta)$ gives a lower bound on the total completion time in expectation of an arbitrary policy $\Pi$, see also Burnetas and Katehakis [21]. That is,

$$
\mathbb{E}[\Pi(\boldsymbol{n}, \boldsymbol{\omega}, \boldsymbol{\alpha})] \geq \sum_{i=1}^{m} \frac{n_{i}\left(n_{i}+1\right)}{2} \frac{\omega_{i}}{\alpha_{i}-1}+\sum_{i=1}^{m-1} \sum_{j=i+1}^{m} n_{i} n_{j} \mathbb{E}\left[\min \left\{\frac{1}{\Theta_{i}}, \frac{1}{\Theta_{j}}\right\}\right],
$$

for any $\boldsymbol{n} \geq \mathbf{0}, \boldsymbol{\omega}>\mathbf{0}$, and $\boldsymbol{\alpha}>\mathbf{1}$. Hereby, note that $\mathbb{E}\left[\Theta_{i}^{-1}\right]=\frac{\omega_{i}}{\alpha_{i}-1}$ for all $i \in\{1, \ldots, m\}$. 
The expectations on the right hand side of (4.11) can be rewritten using the following insight: let $Y=\min \left\{\Theta_{i}^{-1}, \Theta_{j}^{-1}\right\}$ for some $i \neq j$, and let $F_{Y}(\cdot)$ be the corresponding cumulative distribution function. Moreover, let $F_{\Theta_{i}^{-1}}(\cdot)$ denote the cumulative distribution function of random variable $\Theta_{i}^{-1}$, which denotes the processing time of jobs in class $J_{i}$. Then,

$$
\begin{aligned}
1-F_{Y}(y) & =1-\operatorname{Pr}[Y \leq y]=\operatorname{Pr}\left[\Theta_{i}^{-1}>y\right] \operatorname{Pr}\left[\Theta_{j}^{-1}>y\right]=\left(1-F_{\Theta_{i}^{-1}}(y)\right)\left(1-F_{\Theta_{j}^{-1}}(y)\right) \\
& =\left(1-F_{\Theta_{i}^{-1}}(y)\right)+\left(1-F_{\Theta_{j}^{-1}}(y)\right)-\left(1-F_{\Theta_{i}^{-1}}(y) F_{\Theta_{j}^{-1}}(y)\right)
\end{aligned}
$$

such that

$$
\mathbb{E}\left[\min \left\{\Theta_{i}^{-1}, \Theta_{j}^{-1}\right\}\right]=\int_{0}^{\infty}\left(1-F_{Y}(y)\right) d y=\frac{\omega_{i}}{\alpha_{i}-1}+\frac{\omega_{j}}{\alpha_{j}-1}-\int_{0}^{\infty}\left(1-F_{\Theta_{i}^{-1}}(y) F_{\Theta_{j}^{-1}}(y)\right) d y
$$

Working out the last integral, plugging it into (4.11), and rewriting leads to the following result.

Lemma 4.4.1. For any $\boldsymbol{n} \geq \mathbf{0}, \boldsymbol{\omega}>\mathbf{0}$ and $\boldsymbol{\alpha}>\mathbf{1}$ with $\frac{\omega_{1}}{\alpha_{1}-1} \leq \frac{\omega_{2}}{\alpha_{2}-1} \leq \ldots \leq \frac{\omega_{m}}{\alpha_{m}-1}$,

$$
\mathbb{E}\left[\Pi^{*}(\boldsymbol{n}, \boldsymbol{\omega}, \boldsymbol{\alpha})\right] \geq \sum_{i=1}^{m} \frac{n_{i}\left(n_{i}+1\right)}{2} \frac{\omega_{i}}{\alpha_{i}-1}+\sum_{i=1}^{m-1} \sum_{j=i+1}^{m} n_{i} n_{j} \xi(i, j) \frac{\omega_{i}}{\alpha_{i}-1},
$$

where

$$
\xi(i, j)=1-\frac{\left(\frac{\omega_{i}}{\omega_{i}+\omega_{j}}\right)^{\alpha_{i}-1}\left(\frac{\omega_{j}}{\omega_{i}+\omega_{j}}\right)^{\alpha_{j}-1}}{\left(\alpha_{i}+\alpha_{j}-1\right) \cdot B\left(\alpha_{i}, \alpha_{j}\right)} \in(0,1),
$$

and $B\left(\alpha_{i}, \alpha_{j}\right)=\int_{0}^{1} t^{\alpha_{i}-1}(1-t)^{\alpha_{j}-1} d t$ denotes a Beta function.

The proof of this lemma and of all remaining results in this section are presented in Appendix 4.9.2.

In order to derive a better upper bound than (4.7) on the performance of $\ell$-SEPT, we first observe that the performance of $\ell$-SEPT given an arbitrary amount of job classes can be expressed as a linear combination of the performances of $\ell$-SEPT given one and two job classes.

Lemma 4.4.2. For any $n \geq 0, \omega>0$ and $\alpha>1$,

$$
\mathbb{E}\left[\Pi^{\ell}(\boldsymbol{n}, \boldsymbol{\omega}, \boldsymbol{\alpha})\right]=\sum_{i=1}^{m-1} \sum_{j=i+1}^{m} \mathbb{E}\left[\Pi^{\ell}\left(n_{i} \boldsymbol{e}_{i}+n_{j} \boldsymbol{e}_{j}, \boldsymbol{\omega}, \boldsymbol{\alpha}\right)\right]-(m-2) \sum_{i=1}^{m} \mathbb{E}\left[\Pi^{\ell}\left(n_{i} \boldsymbol{e}_{i}, \boldsymbol{\omega}, \boldsymbol{\alpha}\right)\right] .
$$

Based on this lemma and assuming without loss of generality that $\frac{\omega_{1}}{\alpha_{1}-1} \leq \frac{\omega_{2}}{\alpha_{2}-1} \leq \ldots \leq$ $\frac{\omega_{m}}{\alpha_{m}-1}$, we obtain the following upper bound on the performance of $\ell$-SEPT.

Lemma 4.4.3. For any $\boldsymbol{n} \geq \mathbf{0}, \boldsymbol{\omega}>\mathbf{0}$ and $\boldsymbol{\alpha}>\mathbf{1}$ with $\frac{\omega_{1}}{\alpha_{1}-1} \leq \frac{\omega_{2}}{\alpha_{2}-1} \leq \ldots \leq \frac{\omega_{m}}{\alpha_{m}-1}$,

$$
\mathbb{E}\left[\Pi^{\ell}(\boldsymbol{n}, \boldsymbol{\omega}, \boldsymbol{\alpha})\right] \leq \sum_{i=1}^{m} \frac{n_{i}\left(n_{i}+1\right)}{2} \frac{\omega_{i}}{\alpha_{i}-1}+\sum_{i=1}^{m-1} \sum_{j=i+1}^{m}\left[\left(n_{i}-1\right) n_{j} \frac{\omega_{i}}{\alpha_{i}-1} \zeta(i, j)+n_{j} \frac{\omega_{i}}{\alpha_{i}-1}\right],
$$

where

$$
\zeta(i, j)=\left[1-\frac{1}{\alpha_{i}}\left(\frac{\omega_{i}\left(\alpha_{j}-1\right)}{\alpha_{i} \omega_{j}}\right)^{\alpha_{i}-1}\right] \in(0,1)
$$


To obtain this lemma, the following proposition is used.

Proposition 4.4.4. For any $\omega_{i}, \omega_{j}>0$ and $\alpha_{i}, \alpha_{j}>1$,

$$
\int_{0}^{\infty} \min \left\{\frac{\omega_{i}+x_{i}^{1}}{\alpha_{i}}, \frac{\omega_{j}}{\alpha_{j}-1}\right\} f_{i}^{1}\left(x_{i}^{1}\right) d x_{i}^{1}=\frac{\omega_{i}}{\alpha_{i}-1}\left[1-\frac{1}{\alpha_{i}}\left(\frac{\omega_{i}\left(\alpha_{j}-1\right)}{\alpha_{i} \omega_{j}}\right)^{\alpha_{i}-1}\right] .
$$

The proof is omitted, since it follows from a trivial adjustment of the proof of Proposition 4.2.2.

The lower bound in Lemma 4.4.1 and upper bound in Lemma 4.4.3 can be used to investigate the performance of $\ell$-SEPT with respect to OPT. This is done by calculating the ratio of these bounds for several Bayesian scheduling instances. Initially, we assume that there are only 3 job classes. The instances studied are as follows: the gamma prior settings are set such that $\omega_{i},\left(\alpha_{i}-1\right) \in\{0.0001,0.0002,0.0005, \ldots, 100,200,500\}$ for each job class $J_{i}(i \in\{1,2,3\})$. For each of these settings, we further calculate the worst possible ratio over the following numbers of jobs in each class: $n_{1}, n_{2}, n_{3} \in\{10,20,50,100\} \cup\{200,400, \ldots, 1800,2000\}$. This results in almost 240 billion different computations, covering a large amount of the possible parameter settings. All these computations are performed in MATLAB.

Preliminary computations show that $\ell$-SEPT performs relatively good when the a priori expected processing times of the job classes differ significantly, i.e., when

$$
\max _{i}\left\{\frac{\omega_{i}}{\alpha_{i}-1}\right\}>2 \cdot \min _{j}\left\{\frac{\omega_{j}}{\alpha_{j}-1}\right\} \text {. }
$$

Intuitively, learning will only be beneficial in this case with a very small probability such that $\ell$-SEPT and OPT will process the job classes in the same order. Therefore, we focus on the parameter settings with $\omega$ and $\alpha$ opposing (4.12). These are presented in Figure 4.2, where

$$
\alpha_{\max }=\max _{i \in\{1,2,3\}} \alpha_{i}, \quad \alpha_{\text {median }}=\operatorname{median}_{i \in\{1,2,3\}} \alpha_{i} \text {, and } \alpha_{\min }=\min _{i \in\{1,2,3\}} \alpha_{i} .
$$

As can be seen in the figure, we distinguish between 12 different cases, depending on the following points:

- $\alpha_{\min }$ is close to its lower limit (which equals one);

- $\alpha_{\min }$ and $\alpha_{\max }$ are close or not;

- $\alpha_{\text {median }}$ is close to $\alpha_{\min }$, close to $\alpha_{\max }$ or somewhere in between.

The results are presented in Table 4.1. For each case, we report the largest ratio encountered along with the corresponding parameters, and the average ratio. The largest ratio encountered is 2.4097 (case 7 ), which is close to the lower bound of $1+\sqrt{m-1}=1+\sqrt{2} \approx 2.4142$. This ratio is obtained for the setting with $\frac{\omega_{i}}{\alpha_{i}-1}=1$ for all $i$, two job classes being very uncertain ( $\alpha_{2}$ and $\alpha_{3}$ close to one), and one job class very certain ( $\alpha_{1}$ taking the highest value allowed in our settings). Moreover, the corresponding values for $n_{1}, n_{2}$ and $n_{3}$ are as large as possible, while preserving that the number of jobs in the class with certain jobs, $n_{1}$, is roughly a factor of $\sqrt{m-1}=\sqrt{2}$ larger than the number of jobs in the other job classes, $n_{2}$ and $n_{3}$.

Overall, the structure of the instance yielding the highest ratio seems to adhere the exact same structure as in the construction of the lower bound on the performance ratio of $\ell$-SEPT 


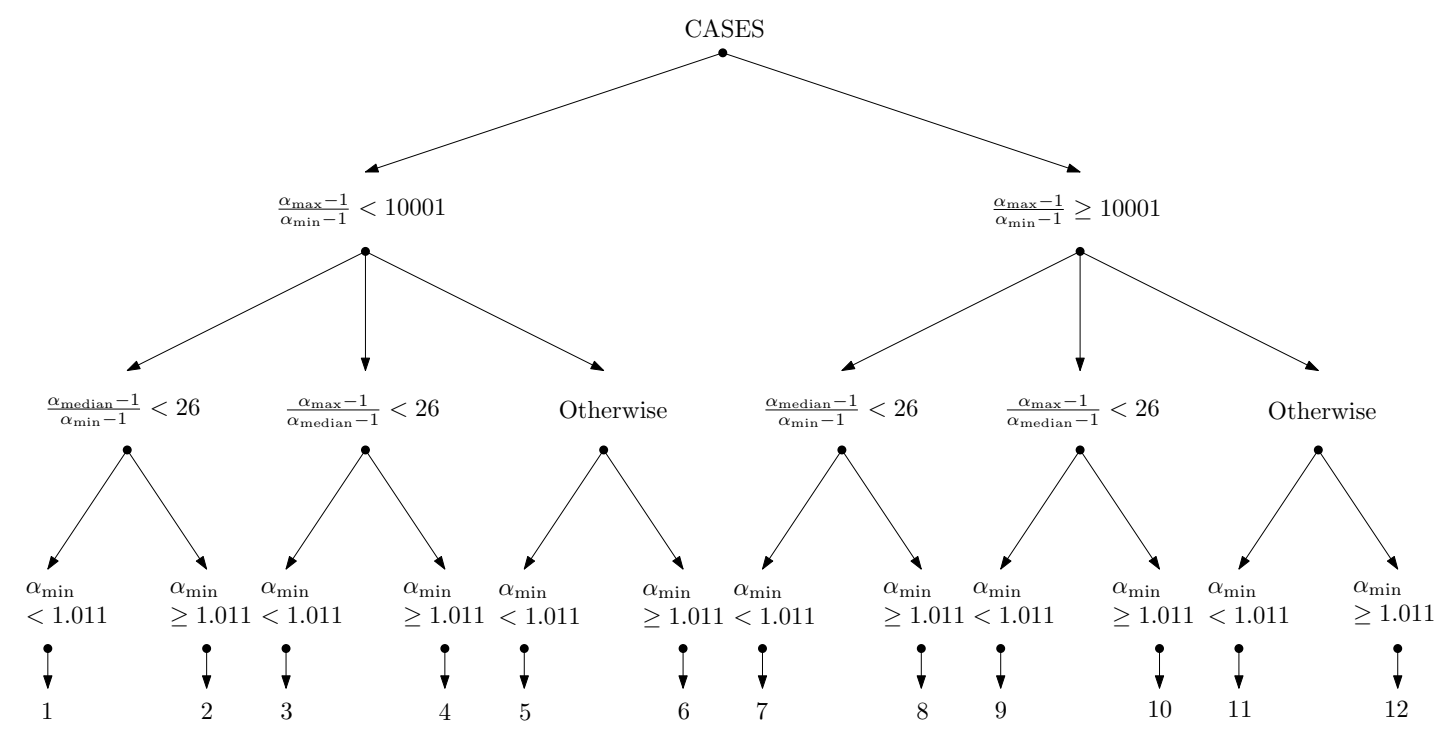

Figure 4.2: 12 different case settings considered

in Section 4.3.2. Moreover, cases 1 and 11, which also return a large ratio, follow this structure as well but within the limitations of their case setting. It therefore comes as no surprise that these cases also yield high performance ratios. Considering the average performance ratios per case, it follows that the average ratio is positively correlated with the ratio $\alpha_{\max } / \alpha_{\min }$ and negatively correlated with $\alpha_{\min }$. Given that $\alpha_{\min }$ is close to one and having a high value for $\alpha_{\max }$, it does not seem to matter that much where $\alpha_{\text {median }}$ is (compare cases 7,9 and 11). To find the worst case instance though, it matters since having a $\alpha_{\text {median }}$ far away from $\alpha_{\min }$ only gives a performance ratio of at most 2.3784 (see settings 9 and 11), which supports our claim.

Intuitively, the computational results are explained as follows. From Lemmas 4.4.1 and 4.4.3, we have that both the lower bound on an optimal policy and the upper bound on $\ell$-SEPT contain the term $\sum_{i=1}^{m} \frac{n_{i}\left(n_{i}+1\right)}{2} \frac{\omega_{i}}{\alpha_{i}-1}$. In order to find the worst case ratio of the upper bound on $\mathbb{E}\left[\Pi^{\ell}(\boldsymbol{n}, \boldsymbol{\omega}, \boldsymbol{\alpha})\right]$ over the lower bound on $\mathbb{E}\left[\Pi^{*}(\boldsymbol{n}, \boldsymbol{\omega}, \boldsymbol{\alpha})\right]$, we therefore need $\xi(i, j)$ and $\zeta(i, j)$ to tend to 0 and 1 , respectively, for almost all combinations of $i \neq j$. This resembles the way in which the lower bound for SEPT in Section 4.3.2 was constructed. For $\xi(i, j)$ to tend to 0, we need that either $\alpha_{i}=\omega_{i}+1$ with $\omega_{i}$ tending to 0 ; or $\alpha_{j}=\omega_{j}+1$ with $\omega_{j}$ tending to 0 . However, for $\zeta(i, j)$ to tend to 1 , we need that either $\alpha_{i}$ or $\alpha_{j}$ tends to infinity.

In the Bayesian scheduling problem with 2 job classes, $J_{1}$ and $J_{2}$, we can establish that $\xi(1,2)$ and $\zeta(1,2)$ tend to 0 and 1 , respectively, by letting $\alpha_{1}$ approach infinity while letting $\omega_{2}=\alpha_{2}-1$ tend to 0 . Confronted with a third class, $J_{3}$, the only way to bring $\xi(2,3)$ to 0 and $\zeta(2,3)$ to 1 is by letting $\alpha_{3}$ approach infinity as well. This will, however, cause $\xi(1,3)$ to tend to 1 , thereby increasing the denominator of the ratio, which is undesirable. Therefore, it seems that the best one can do is to set $\omega_{j}=\alpha_{j}-1$, while letting $\omega_{j}$ tend to 0 , for all classes $j \geq 2$. Within this structure $\xi(i, j)=0$ for all $i \neq j, \zeta(1, j)=1$ for all $j \geq 2$ and $\zeta(i, j)=0$ for all $i, j \geq 2$. The preceeding intuition extends to the case where the number of classes is beyond three. In the resulting structure there is one class for which $\alpha_{1}$ tends to infinity whereas for all other classes $j \geq 2, \omega_{j}=\alpha_{j}-1$, while $\omega_{j}$ tends to 0 . 


\begin{tabular}{|c|c|ccccccccc|c|}
\hline Case & $\rho_{\max }$ & $\omega_{1}$ & $\omega_{2}$ & $\omega_{3}$ & $\alpha_{1}$ & $\alpha_{2}$ & $\alpha_{3}$ & $n_{1}$ & $n_{2}$ & $n_{3}$ & $\bar{\rho}$ \\
\hline 01 & 2.34 & 5 & 0.0005 & 0.0005 & 11 & 1.001 & 1.001 & 2000 & 1400 & 1400 & 1.21 \\
02 & 2.06 & 0.2 & 0.0001 & 0.0001 & 51 & 1.02 & 1.02 & 1600 & 1000 & 1000 & 1.26 \\
03 & 2.10 & 5 & 0.2 & 0.0005 & 6 & 1.2 & 1.0005 & 1800 & 1000 & 1800 & 1.61 \\
04 & 1.87 & 2 & 0.2 & 0.005 & 11 & 2 & 1.02 & 1200 & 800 & 1400 & 1.36 \\
05 & 2.17 & 1 & 0.0001 & 0.005 & 6 & 1.0005 & 1.02 & 1800 & 1400 & 1000 & 1.77 \\
06 & 1.87 & 1 & 0.02 & 0.0005 & 51 & 2 & 1.02 & 1200 & 800 & 1400 & 1.55 \\
07 & 2.41 & 500 & 0.0001 & 0.0001 & 501 & 1.0001 & 1.0001 & 2000 & 1400 & 1400 & 1.79 \\
08 & 2.06 & 100 & 0.005 & 0.005 & 501 & 1.02 & 1.02 & 1600 & 1000 & 1000 & 1.51 \\
09 & 2.11 & 50 & 2 & 0.001 & 6 & 1.2 & 1.0001 & 1800 & 1000 & 1800 & 1.87 \\
10 & 1.79 & 100 & 10 & 0.005 & 501 & 51 & 1.02 & 800 & 800 & 1400 & 1.69 \\
11 & 2.38 & 500 & 0.005 & 0.0001 & 501 & 1.005 & 1.0001 & 1400 & 1000 & 1000 & 1.87 \\
12 & 1.87 & 100 & 0.2 & 0.005 & 501 & 2 & 1.02 & 1200 & 800 & 1400 & 1.69 \\
\hline
\end{tabular}

Table 4.1: Largest and average performance guarantees of $\ell$-SEPT for 12 different cases

With respect to the number of jobs in each job class, we can say the following: by choosing $n_{1}$ bigger than $n_{j}$ for all $j \geq 2$, we give some extra weight to $\zeta(1, j)$ in the numerator. Increasing the number of jobs in class $J_{1}$, however, also increases the term $\frac{n_{1}\left(n_{1}+1\right)}{2}$ in the denominator, thereby decreasing the ratio again. Due to this trade-off, the optimal choice is $n_{1}=n \sqrt{m-1}$ and $n_{j}=n$ for all $j \geq 2$, with $n$ going to infinity. This explains why we found that $n_{1}$, is roughly a factor of $\sqrt{m-1}=\sqrt{2}$ larger than $n_{2}$ and $n_{3}$.

We remark that in the intuitive explanation above only extreme values for the job class parameters are considered. That is, we either choose $\alpha_{i}$ to tend to 1 or infinity. Reason for this is that choosing $\alpha_{i}$ somewhere in the middle of its range seems not to lead to a large upper bound on the ratio of $\mathbb{E}\left[\Pi^{\ell}(\boldsymbol{n}, \boldsymbol{\omega}, \boldsymbol{\alpha})\right]$ over $\mathbb{E}\left[\Pi^{*}(\boldsymbol{n}, \boldsymbol{\omega}, \boldsymbol{\alpha})\right]$. In that case, $\xi(i, j)$ will be either significantly larger than 0 or $\zeta(i, j)$ significantly smaller than 1 , depending on how the parameters of job class $j \neq i$ are chosen. The computational results given in Table 4.1 approve the reasoning above.

So far we only considered the ratio of the upper bound in Lemma 4.4.1 over the lower bound in Lemma 4.4.3 given 3 job classes. To conclude this subsection, we will therefore have a brief look at the behavior of this ratio in case there are more than 3 job classes. To do this, we focus on the case setting that previously, in the presence of 3 job classes, gave the largest ratio, i.e., case 7 :

$$
\begin{aligned}
\alpha_{1}-1=\omega_{1} & =500, \\
n_{1} & =2000,
\end{aligned} \quad \begin{aligned}
\alpha_{2}-1=\omega_{2} & =\ldots=\alpha_{m}-1=\alpha_{m}=0.0001, \\
n_{2} & =\ldots=n_{m}=1400,
\end{aligned}
$$

and an adjusted version of this case:

$$
\begin{aligned}
\alpha_{1}-1=\omega_{1} & =10000, \\
n_{1} & =\sqrt{m-1} \cdot 10000,
\end{aligned} \quad \begin{aligned}
\alpha_{2}-1=\omega_{2} & =\ldots=\alpha_{m}-1=\alpha_{m}=0.0001, \\
n_{2} & =\ldots=n_{m}=10000,
\end{aligned}
$$

and compute the ratios for $m \in\{3, \ldots, 200\}$. The results are given in Figure 4.3. 


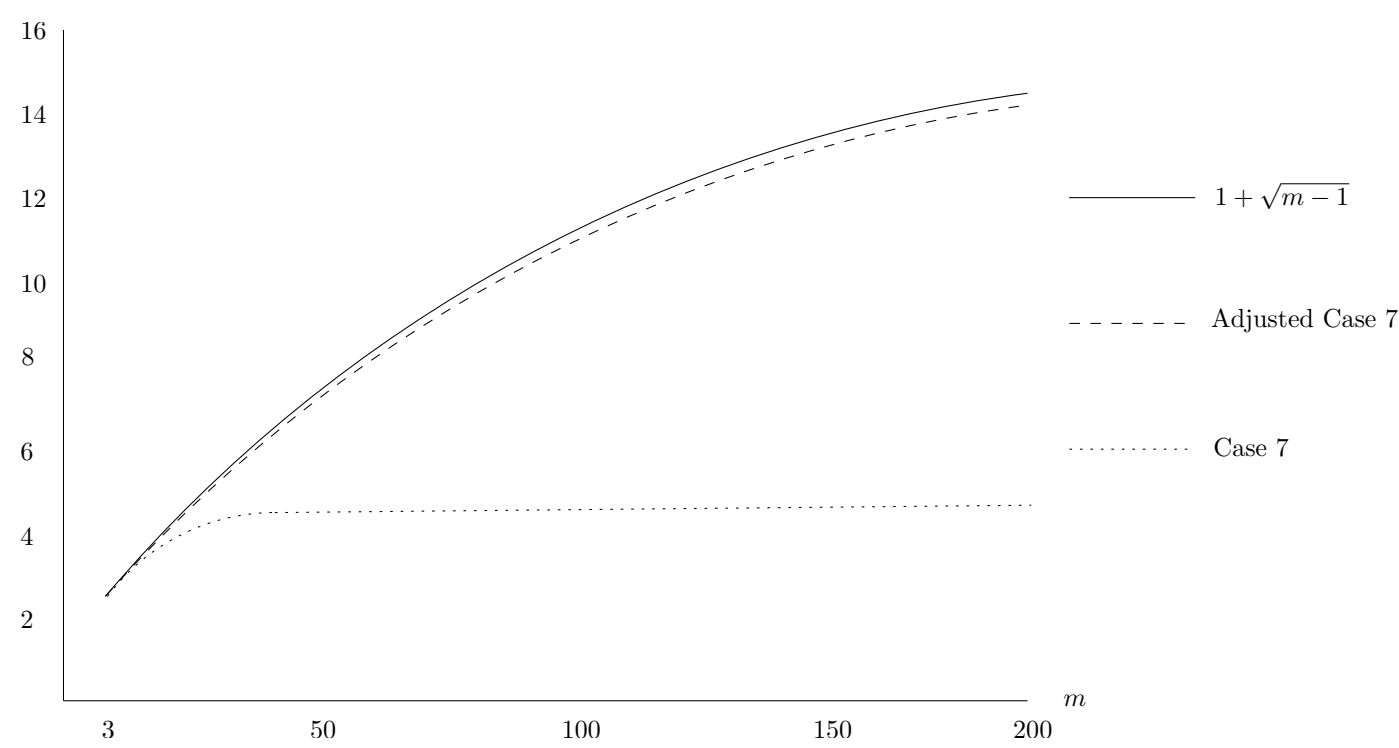

Figure 4.3: Largest ratio for case setting 7 with more than 3 job classes

We observe that the computed ratios for the case 7 setting are very close to $1+\sqrt{m-1}$ for small number of job classes $m$. As soon as $m$ increases, a more extreme setting is necessary to still get close $1+\sqrt{m-1}$. This setting is provided by the adjusted version of case 7 , which is in agreement with the construction of the lower bound for SEPT in Section 4.3.2. For both case settings, it however holds that the largest ratio is smaller than $1+\sqrt{m-1}$ for every number of job classes $m$.

The preceding computations and observations lead us to the following conjecture.

Conjecture 4.4.5. For any $n \geq 0, \omega>0$ and $\alpha>1$,

$$
\frac{\mathbb{E}\left[\Pi^{\ell}(\boldsymbol{n}, \boldsymbol{\omega}, \boldsymbol{\alpha})\right]}{\mathbb{E}\left[\Pi^{*}(\boldsymbol{n}, \boldsymbol{\omega}, \boldsymbol{\alpha})\right]} \leq 1+\sqrt{m-1} .
$$

\subsection{Problem Setting II}

By choosing $m=2$ in the Section 4.3, we obtain a performance guarantee of 2 for both SEPT and $\ell$-SEPT in Problem Setting II. However, this result can be strengthened, which is done in this section. By giving a better upper bound on the performance guarantees of SEPT and $\ell$-SEPT and by improving the result in Lemma 4.3.5, we can give a performance guarantee that depends fully on the number of jobs in both job classes, $n_{1}$ and $n_{2}$, and is strictly less than 2 , but tends to 2 when $n_{1}$ and $n_{2}$ tend to infinity.

\subsubsection{Upper bound on performance guarantees}

In this subsection, we give an upper bound on the performance of SEPT and $\ell$-SEPT with respect to the performance of OPT. First, we give a lower bound on the value of the optimal policy, which is derived from the fact that the expected completion time of each job is delayed by the expected processing time of the first job to be processed by the optimal policy. 
Lemma 4.5.1. For any $n_{1}, n_{2} \geq 0, \boldsymbol{\omega}>\mathbf{0}$, and $\boldsymbol{\alpha}>\mathbf{1}$,

$\mathbb{E}\left[\Pi^{*}\left(n_{1}, n_{2}, \boldsymbol{\omega}, \boldsymbol{\alpha}\right)\right] \geq \frac{n_{1}\left(n_{1}+1\right)}{2} \frac{\omega_{1}}{\alpha_{1}-1}+\frac{n_{2}\left(n_{2}+1\right)}{2} \frac{\omega_{2}}{\alpha_{2}-1}+\min \left\{n_{1}, n_{2}\right\} \min \left\{\frac{\omega_{1}}{\alpha_{1}-1}, \frac{\omega_{2}}{\alpha_{2}-1}\right\}$.

Proof. Let $n_{1}, n_{2} \geq \mathbf{0}, \boldsymbol{\omega}>\mathbf{0}$, and $\boldsymbol{\alpha}>\mathbf{1}$. Then,

$$
\begin{gathered}
\mathbb{E}\left[\Pi^{*}\left(n_{1}, n_{2}, \boldsymbol{\omega}, \boldsymbol{\alpha}\right)\right]=\min \left\{\left(n_{1}+n_{2}\right) \frac{\omega_{1}}{\alpha_{1}-1}+\int_{0}^{\infty} \mathbb{E}\left[\Pi^{*}\left(n_{1}-1, n_{2}, \boldsymbol{\omega}+x_{1}^{1} \boldsymbol{e}_{1}, \boldsymbol{\alpha}+\boldsymbol{e}_{1}\right)\right] f_{1}^{1}\left(x_{1}^{1}\right) d x_{1}^{1} ;\right. \\
\left.\quad\left(n_{1}+n_{2}\right) \frac{\omega_{2}}{\alpha_{2}-1}+\int_{0}^{\infty} \mathbb{E}\left[\Pi^{*}\left(n_{1}, n_{2}-1, \boldsymbol{\omega}+x_{2}^{1} \boldsymbol{e}_{2}, \boldsymbol{\alpha}+\boldsymbol{e}_{2}\right)\right] f_{2}^{1}\left(x_{2}^{1}\right) d x_{2}^{1}\right\} \\
\geq \min \left\{\left(n_{1}+n_{2}\right) \frac{\omega_{1}}{\alpha_{1}-1}+\frac{\left(n_{1}-1\right) n_{1}}{2} \frac{\omega_{1}}{\alpha_{1}-1}+\frac{n_{2}\left(n_{2}+1\right)}{2} \frac{\omega_{2}}{\alpha_{2}-1} ;\right. \\
\left.\quad\left(n_{1}+n_{2}\right) \frac{\omega_{2}}{\alpha_{2}-1}+\frac{n_{1}\left(n_{1}+1\right)}{2} \frac{\omega_{1}}{\alpha_{1}-1}+\frac{\left(n_{2}-1\right) n_{2}}{2} \frac{\omega_{2}}{\alpha_{2}-1}\right\} \\
=\frac{n_{1}\left(n_{1}+1\right)}{2} \frac{\omega_{1}}{\alpha_{1}-1}+\frac{n_{2}\left(n_{2}+1\right)}{2} \frac{\omega_{2}}{\alpha_{2}-1}+\min \left\{n_{2} \frac{\omega_{1}}{\alpha_{1}-1}, n_{1} \frac{\omega_{2}}{\alpha_{2}-1}\right\} \\
\geq \frac{n_{1}\left(n_{1}+1\right)}{2} \frac{\omega_{1}}{\alpha_{1}-1}+\frac{n_{2}\left(n_{2}+1\right)}{2} \frac{\omega_{2}}{\alpha_{2}-1}+\min \left\{n_{1}, n_{2}\right\} \min \left\{\frac{\omega_{1}}{\alpha_{1}-1}, \frac{\omega_{2}}{\alpha_{2}-1}\right\},
\end{gathered}
$$

where the first inequality follows from Lemma 4.3.1.

Theorem 4.5.2. For any $n_{1}, n_{2} \geq 0, \boldsymbol{\omega}>\mathbf{0}$, and $\boldsymbol{\alpha}>\mathbf{1}$,

$$
\frac{\mathbb{E}\left[\Pi^{\ell}\left(n_{1}, n_{2}, \boldsymbol{\omega}, \boldsymbol{\alpha}\right)\right]}{\mathbb{E}\left[\Pi^{*}\left(n_{1}, n_{2}, \boldsymbol{\omega}, \boldsymbol{\alpha}\right)\right]} \leq \frac{\mathbb{E}\left[\Pi^{s}\left(n_{1}, n_{2}, \boldsymbol{\omega}, \boldsymbol{\alpha}\right)\right]}{\mathbb{E}\left[\Pi^{*}\left(n_{1}, n_{2}, \boldsymbol{\omega}, \boldsymbol{\alpha}\right)\right]} \leq \frac{n_{1}^{2}+n_{2}^{2}+2 n_{1} n_{2}+n_{1}+n_{2}}{n_{1}^{2}+n_{2}^{2}+n_{1}+n_{2}+2 \min \left\{n_{1}, n_{2}\right\}} .
$$

Proof. The first inequality follows directly from Theorem 4.3.2. To prove the second and last inequality, let $n_{1}, n_{2} \geq 0, \boldsymbol{\omega}>\mathbf{0}$, and $\boldsymbol{\alpha}>\mathbf{1}$. Combining (4.5) and Lemma 4.5.1, we obtain

$$
\frac{\mathbb{E}\left[\Pi^{s}\left(n_{1}, n_{2}, \boldsymbol{\omega}, \boldsymbol{\alpha}\right)\right]}{\mathbb{E}\left[\Pi^{*}\left(n_{1}, n_{2}, \boldsymbol{\omega}, \boldsymbol{\alpha}\right)\right]} \leq \frac{n_{1}\left(n_{1}+1\right) \frac{\omega_{1}}{\alpha_{1}-1}+n_{2}\left(n_{2}+1\right) \frac{\omega_{2}}{\alpha_{2}-1}+2 n_{1} n_{2} \min \left\{\frac{\omega_{1}}{\alpha_{1}-1}, \frac{\omega_{2}}{\alpha_{2}-1}\right\}}{n_{1}\left(n_{1}+1\right) \frac{\omega_{1}}{\alpha_{1}-1}+n_{2}\left(n_{2}+1\right) \frac{\omega_{2}}{\alpha_{2}-1}+2 \min \left\{n_{1}, n_{2}\right\} \min \left\{\frac{\omega_{1}}{\alpha_{1}-1}, \frac{\omega_{2}}{\alpha_{2}-1}\right\}} .
$$

Observing that for any $0<c \leq b$ and $0<d \leq a$, it holds that $\frac{a+b}{a+c} \leq \frac{d+b}{d+c}$ and replacing $\frac{\omega_{1}}{\alpha_{1}-1}$ and $\frac{\omega_{2}}{\alpha_{2}-1}$ by the minimum of the two, we can bound this by

$$
\leq \frac{n_{1}^{2}+n_{2}^{2}+2 n_{1} n_{2}+n_{1}+n_{2}}{n_{1}^{2}+n_{2}^{2}+n_{1}+n_{2}+2 \min \left\{n_{1}, n_{2}\right\}}
$$

From Theorem 4.5.2, it follows that the performance guarantees of SEPT and $\ell$-SEPT will be close to one in case the number of jobs in one class is of a different order than the number of jobs in the second class. To be more explicit, when the number of jobs in one class is fixed while the number of jobs in the second class tends to infinity, then the performance guarantee will go to one, yielding asymptotic optimality of SEPT and $\ell$-SEPT. 


\subsubsection{Tightness of performance guarantees}

We show that for any $\epsilon>0$ there exists an instance for which the ratio of the value of SEPT to the value of OPT is only an additive $\epsilon$ away from the performance guarantee of Theorem 4.5.2. The intuition behind this instance is identical to the one given in Section 4.3.2: we set the prior parameters in such a way that SEPT is forced to start with class $J_{1}$, while OPT first processes several jobs of all other classes. We will therefore again use the lower bound on SEPT in Lemma 4.3.4, and the upper bound on OPT denoted below, which is a slightly better version of the bound in Lemma 4.3.5.

Lemma 4.5.3. For any $n_{1}, n_{2} \geq 0$, there exist parameter settings $\boldsymbol{\omega}>\mathbf{0}$, and $\boldsymbol{\alpha}>1$ such that $\frac{\omega_{1}}{\alpha_{1}-1}<\frac{\omega_{2}}{\alpha_{2}-1}=1$ and

$$
\mathbb{E}\left[\Pi^{*}\left(n_{1}, n_{2}, \boldsymbol{\omega}, \boldsymbol{\alpha}\right)\right]<n_{1}+\frac{n_{1}\left(n_{1}+1\right)}{2}+\frac{n_{2}\left(n_{2}+1\right)}{2}+\epsilon,
$$

for any $\epsilon>0$.

Proof. Consider the following policy $\Pi$ : first process one job of class $J_{2}$, observing realization $y$, and schedule all remaining jobs according to SEPT. That is, if $\frac{\omega_{1}}{\alpha_{1}-1} \leq \frac{\omega_{2}+y}{\alpha_{2}}$ then process first all jobs of class $J_{1}$ and then the remaining jobs of class $J_{2}$ and otherwise first process the remaining jobs of class $J_{2}$ and then all jobs of class $J_{1}$. For any $n_{1}, n_{2} \geq 0, \boldsymbol{\omega}>\mathbf{0}$, and $\boldsymbol{\alpha}>\mathbf{1}$ such that $\frac{\omega_{1}}{\alpha_{1}-1}<\frac{\omega_{2}}{\alpha_{2}-1}=1$, we have

$$
\begin{aligned}
& \mathbb{E}\left[\Pi^{*}\left(n_{1}, n_{2}, \boldsymbol{\omega}, \boldsymbol{\alpha}\right)\right] \leq \mathbb{E}\left[\Pi\left(n_{1}, n_{2}, \boldsymbol{\omega}, \boldsymbol{\alpha}\right)\right] \\
& =\left(n_{1}+n_{2}\right) \frac{\omega_{2}}{\alpha_{2}-1}+\int_{0}^{\infty} \mathbb{E}\left[\Pi^{s}\left(n_{1}, n_{2}-1, \boldsymbol{\omega}+x_{2}^{1} \boldsymbol{e}_{2}, \boldsymbol{\alpha}+\boldsymbol{e}_{2}\right)\right] f_{2}^{1}\left(x_{2}^{1}\right) d x_{2}^{1} \\
& \stackrel{\left(\mathrm{CH}^{4}\right)}{=} n_{1}+n_{2}+\frac{n_{1}\left(n_{1}+1\right)}{2} \frac{\omega_{1}}{\alpha_{1}-1}+\frac{n_{2}\left(n_{2}-1\right)}{2} \\
& \quad+n_{1}\left(n_{2}-1\right) \int_{0}^{\infty} \min \left\{\frac{\omega_{1}}{\alpha_{1}-1}, \frac{\omega_{2}+x_{2}^{1}}{\alpha_{2}}\right\} f_{2}^{1}\left(x_{2}^{1}\right) d x_{2}^{1} \\
& \quad<n_{1}+\frac{n_{1}\left(n_{1}+1\right)}{2}+\frac{n_{2}\left(n_{2}+1\right)}{2}+n_{1}\left(n_{2}-1\right) \int_{0}^{\infty} \min \left\{1, \frac{\omega_{2}+x_{2}^{1}}{\alpha_{2}}\right\} f_{2}^{1}\left(x_{2}^{1}\right) d x_{2}^{1} \\
& \stackrel{\text { Prop. }^{\prime} .2 .2}{=} n_{1}+\frac{n_{1}\left(n_{1}+1\right)}{2}+\frac{n_{2}\left(n_{2}+1\right)}{2}+n_{1}\left(n_{2}-1\right)\left[\frac{\omega_{2}}{\alpha_{2}-1}-\frac{1}{\alpha_{2}-1}\left(\frac{\omega_{2}}{\alpha_{2}}\right)^{\alpha_{2}}\right] .
\end{aligned}
$$

Recall that by assumption $\omega_{2}=\alpha_{2}-1$. Combining (4.13) and Proposition 4.2.1, and letting $\alpha_{2}$ tend to 1 from above, we find

$$
\lim _{\alpha_{2} \downarrow 1} \mathbb{E}\left[\Pi^{*}\left(n_{1}, n_{2}, \boldsymbol{\omega}, \boldsymbol{\alpha}\right)\right]<n_{1}+\frac{n_{1}\left(n_{1}+1\right)}{2}+\frac{n_{2}\left(n_{2}+1\right)}{2} .
$$

Hence, it follows that for any $n_{1}, n_{2}, \omega_{1}<\alpha_{1}-1$, there exists for any $\epsilon>0$ an $\alpha^{*}>1$ such that for all $1<\alpha_{2}=\omega_{2}+1<\alpha^{*}$

$$
\mathbb{E}\left[\Pi^{*}\left(n_{1}, n_{2}, \boldsymbol{\omega}, \boldsymbol{\alpha}\right)\right]<n_{1}+\frac{n_{1}\left(n_{1}+1\right)}{2}+\frac{n_{2}\left(n_{2}+1\right)}{2}+\epsilon .
$$


The restrictions imposed on the parameters $\omega_{1}, \omega_{2}, \alpha_{1}$, and $\alpha_{2}$ in Lemmas 4.3.4 and 4.5.3 can be satisfied simultaneously. Hence, we obtain the theorem below as a straightforward consequence of these Lemmata.

Theorem 4.5.4. There exist parameter settings $n_{1}, n_{2} \geq 0, \boldsymbol{\omega}>\mathbf{0}, \boldsymbol{\alpha}>\mathbf{1}$ such that

$$
\frac{\mathbb{E}\left[\Pi^{s}\left(n_{1}, n_{2}, \boldsymbol{\omega}, \boldsymbol{\alpha}\right)\right]}{\mathbb{E}\left[\Pi^{*}\left(n_{1}, n_{2}, \boldsymbol{\omega}, \boldsymbol{\alpha}\right)\right]}>\frac{n_{1}^{2}+n_{2}^{2}+2 n_{1} n_{2}+n_{1}+n_{2}}{n_{1}^{2}+n_{2}^{2}+3 n_{1}+n_{2}}-\epsilon
$$

for any $\epsilon>0$.

The same lower bound can be found for the performance of $\ell$-SEPT. The intuition of this result is similar to the one behind Theorem 4.3.7. That is, we adjust the structure of the bad instance used to obtain the lower bound on the performance guarantee of SEPT (Theorem 4.5.4) in such a way that $\ell$-SEPT does not switch to processing jobs from the another class $J_{i}(i \in\{2, \ldots, m\})$ after it processed a few jobs of class $J_{1}$. As mentioned before, this is done by setting the values of $\omega_{1}$ and $\alpha_{1}$ extremely large such that we are very sure about the value of $\vartheta_{1}$.

Theorem 4.5.5. There exist parameter settings $n_{1}, n_{2} \geq 0, \boldsymbol{\omega}>\mathbf{0}, \boldsymbol{\alpha}>\mathbf{1}$ such that

$$
\frac{\mathbb{E}\left[\Pi^{\ell}\left(n_{1}, n_{2}, \boldsymbol{\omega}, \boldsymbol{\alpha}\right)\right]}{\mathbb{E}\left[\Pi^{*}\left(n_{1}, n_{2}, \boldsymbol{\omega}, \boldsymbol{\alpha}\right)\right]}>\frac{n_{1}^{2}+n_{2}^{2}+2 n_{1} n_{2}+n_{1}+n_{2}}{n_{1}^{2}+n_{2}^{2}+3 n_{1}+n_{2}}-\epsilon
$$

for any $\epsilon>0$.

A formal proof of this theorem is given in Appendix 4.9.3.

\subsection{Problem Setting III}

In this section, we consider again the Bayesian scheduling problem with 2 job classes. However, we assume that the parameter of the processing time distribution of job class $J_{2}$ is known instead. We therefore use the exact same notation as in the previous section, except for the following: let $\vartheta$ and $\mu$ denote the unknown and known parameter of job class $J_{1}$ and $J_{2}$, respectively. Further, we drop the index of $\omega_{1}$ and $\alpha_{1}$, leave $\mu$ out of the state vector $\left(n_{1}, n_{2}, \omega, \alpha\right)$, and write $x$ and $f(x)$ instead of $x_{1}^{1}$ and $f_{1}^{1}\left(x_{1}^{1}\right)$. Note that $\mathbb{E}\left[X_{2}\right]=\frac{1}{\mu}$, since $X_{2}$ is an exponentially distributed random variable.

\subsubsection{Bounds on scheduling policies}

In this subsection, we present several useful bounds on the performance of the scheduling policies. First, we restate Lemma 4.3.1 for Problem Setting III.

Lemma 4.6.1. Let $\Pi$ be an arbitrary scheduling policy. Then, for any $n_{1}, n_{2} \geq 0, \mu, \omega>0$ and $\alpha>1$,

$$
\mathbb{E}\left[\Pi\left(n_{1}, n_{2}, \omega, \alpha\right)\right] \geq \frac{n_{1}\left(n_{1}+1\right)}{2} \frac{\omega}{\alpha-1}+\frac{n_{2}\left(n_{2}+1\right)}{2} \frac{1}{\mu} .
$$


Whenever SEPT or $\ell$-SEPT processes a job of class $J_{2}$, the expected processing times of neither class are updated, causing the policy to process one more job of class $J_{2}$, if available.

Observation 4.6.2. SEPT and $\ell-S E P T$ process all jobs of class $J_{2}$ consecutively.

Together with equation (4.5) and the proof of Theorem 4.3.2 with $m=2$, Observation 4.6.2 shows that the adaptive policy is also better than sequencing the jobs a priori in Problem Setting III.

Corollary 4.6.3. For any $n_{1}, n_{2} \geq 0, \mu, \omega>0$, and $\alpha>1$,

$$
\begin{aligned}
\mathbb{E}\left[\Pi^{\ell}\left(n_{1}, n_{2}, \omega, \alpha\right)\right] & \leq \frac{n_{1}\left(n_{1}+1\right)}{2} \frac{\omega}{\alpha-1}+\frac{n_{2}\left(n_{2}+1\right)}{2} \frac{1}{\mu}+n_{1} n_{2} \min \left\{\frac{1}{\mu}, \frac{\omega}{\alpha-1}\right\} \\
& =\mathbb{E}\left[\Pi^{s}\left(n_{1}, n_{2}, \omega, \alpha\right)\right] .
\end{aligned}
$$

We finally present a recursive upper bound on the performance of an optimal policy.

Lemma 4.6.4. For any $n_{1} \geq 0, n_{2} \geq 2, \mu, \omega>0$, and $\alpha>1$,

$$
\begin{aligned}
\mathbb{E}\left[\Pi^{*}\left(n_{1}, n_{2}, \omega, \alpha\right)\right] & -\mathbb{E}\left[\Pi^{*}\left(n_{1}, n_{2}-1, \omega, \alpha\right)\right] \\
& \leq \mathbb{E}\left[\Pi^{*}\left(n_{1}, n_{2}-1, \omega, \alpha\right)\right]-\mathbb{E}\left[\Pi^{*}\left(n_{1}, n_{2}-2, \omega, \alpha\right)\right]+\frac{1}{\mu}
\end{aligned}
$$

Proof. We provide a proof by induction on $n_{1}$. In case $n_{1}=0$, it follows immediately that

$$
\begin{aligned}
\mathbb{E}\left[\Pi^{*}\left(0, n_{2}-1, \omega, \alpha\right)\right] & -\mathbb{E}\left[\Pi^{*}\left(0, n_{2}-2, \omega, \alpha\right)\right]+\frac{1}{\mu}=\frac{n_{2}\left(n_{2}+1\right)}{2} \frac{1}{\mu}-\frac{\left(n_{2}-1\right) n_{2}}{2} \frac{1}{\mu} \\
& =\mathbb{E}\left[\Pi^{*}\left(0, n_{2}, \omega, \alpha\right)\right]-\mathbb{E}\left[\Pi^{*}\left(0, n_{2}-1, \omega, \alpha\right)\right]
\end{aligned}
$$

for any $n_{2} \geq 2, \mu, \omega>0$, and $\alpha>1$. Now, assume that

$$
\begin{aligned}
\mathbb{E}\left[\Pi^{*}\left(n, n_{2}, \omega, \alpha\right)\right] & -\mathbb{E}\left[\Pi^{*}\left(n, n_{2}-1, \omega, \alpha\right)\right] \\
& \leq \mathbb{E}\left[\Pi^{*}\left(n, n_{2}-1, \omega, \alpha\right)\right]-\mathbb{E}\left[\Pi^{*}\left(n, n_{2}-2, \omega, \alpha\right)\right]+\frac{1}{\mu}
\end{aligned}
$$

for all $n \leq n_{1}-1$ and any $n_{2} \geq 2, \mu, \omega>0$ and $\alpha>1$. We will verify that this statement is also valid for $n=n_{1}$. In order to do so, we first consider the case $\mathbb{E}\left[\Pi_{2}^{*}\left(n_{1}, n_{2}-1, w, a\right)\right]<$ $\mathbb{E}\left[\Pi_{1}^{*}\left(n_{1}, n_{2}-1, w, a\right)\right]$. Then,

$$
\begin{aligned}
\mathbb{E}\left[\Pi^{*}\left(n_{1}, n_{2}, \omega, \alpha\right)\right] & -\mathbb{E}\left[\Pi^{*}\left(n_{1}, n_{2}-1, \omega, \alpha\right)\right] \\
& \leq \mathbb{E}\left[\Pi_{2}^{*}\left(n_{1}, n_{2}, \omega, \alpha\right)\right]-\mathbb{E}\left[\Pi_{2}^{*}\left(n_{1}, n_{2}-1, \omega, \alpha\right)\right] \\
& =\mathbb{E}\left[\Pi^{*}\left(n_{1}, n_{2}-1, \omega, \alpha\right)\right]-\mathbb{E}\left[\Pi^{*}\left(n_{1}, n_{2}-2, \omega, \alpha\right)\right]+\frac{1}{\mu},
\end{aligned}
$$

where the inequality follows from the assumption and the optimality of $\Pi^{*}$. Secondly, consider 
the case $\mathbb{E}\left[\Pi_{2}^{*}\left(n_{1}, n_{2}-1, \omega, \alpha\right)\right] \geq \mathbb{E}\left[\Pi_{1}^{*}\left(n_{1}, n_{2}-1, \omega, \alpha\right)\right]$. This gives

$$
\begin{aligned}
\mathbb{E}\left[\Pi^{*}\left(n_{1}, n_{2}, \omega, \alpha\right)\right]- & \mathbb{E}\left[\Pi^{*}\left(n_{1}, n_{2}-1, \omega, \alpha\right)\right] \\
\leq & \mathbb{E}\left[\Pi_{1}^{*}\left(n_{1}, n_{2}, \omega, \alpha\right)\right]-\mathbb{E}\left[\Pi_{1}^{*}\left(n_{1}, n_{2}-1, \omega, \alpha\right)\right] \\
= & \int_{0}^{\infty}\left(\mathbb{E}\left[\Pi^{*}\left(n_{1}-1, n_{2}, \omega+x, \alpha+1\right)\right]+\left(n_{1}+n_{2}\right) \frac{\omega+x}{\alpha}\right) f(x) d x \\
& -\int_{0}^{\infty}\left(\mathbb{E}\left[\Pi^{*}\left(n_{1}-1, n_{2}-1, \omega+x, \alpha+1\right)\right]+\left(n_{1}+n_{2}-1\right) \frac{\omega+x}{\alpha}\right) f(x) d x \\
\leq & \int_{0}^{\infty} \mathbb{E}\left[\Pi^{*}\left(n_{1}-1, n_{2}-1, \omega+x, \alpha+1\right)\right] f(x) d x \\
& -\int_{0}^{\infty}\left(\mathbb{E}\left[\Pi^{*}\left(n_{1}-1, n_{2}-2, \omega+x, \alpha+1\right)\right]-\frac{\omega+x}{\alpha}\right) f(x) d x+\frac{1}{\mu} \\
= & \mathbb{E}\left[\Pi_{1}^{*}\left(n_{1}, n_{2}-1, \omega, \alpha\right)\right]-\mathbb{E}\left[\Pi_{1}^{*}\left(n_{1}, n_{2}-2, \omega, \alpha\right)\right]+\frac{1}{\mu} \\
\leq & \mathbb{E}\left[\Pi^{*}\left(n_{1}, n_{2}-1, \omega, \alpha\right)\right]-\mathbb{E}\left[\Pi^{*}\left(n_{1}, n_{2}-2, \omega, \alpha\right)\right]+\frac{1}{\mu},
\end{aligned}
$$

where the first and last inequality follow from the assumption and the optimality of $\Pi^{*}$, and the second inequality from the induction step.

\subsubsection{Structural analysis of OPT}

In this subsection, we show that SEPT, $\ell$-SEPT, and OPT produce the same schedule in case OPT starts by processing a job of class $J_{2}$. This result builds upon the property of OPT processing all jobs of class $J_{2}$ consecutively. To establish this property, we first show that in case it is optimal to start processing a job of class $J_{1}$, it remains optimal to start processing a job of class $J_{1}$ when the number of jobs in class $J_{2}$ is increased by an arbitrary number.

Lemma 4.6.5. For any $n_{1} \geq 0, n_{2} \geq 1, \mu, \omega>0$, and $\alpha>1$, if

$$
\mathbb{E}\left[\Pi_{2}^{*}\left(n_{1}, n_{2}-1, \omega, \alpha\right)\right] \geq \mathbb{E}\left[\Pi_{1}^{*}\left(n_{1}, n_{2}-1, \omega, \alpha\right)\right],
$$

then

$$
\mathbb{E}\left[\Pi_{2}^{*}\left(n_{1}, n_{2}, \omega, \alpha\right)\right] \geq \mathbb{E}\left[\Pi_{1}^{*}\left(n_{1}, n_{2}, \omega, \alpha\right)\right] .
$$

Proof. Assume that

$$
\mathbb{E}\left[\Pi_{2}^{*}\left(n_{1}, n_{2}-1, \omega, \alpha\right)\right] \geq \mathbb{E}\left[\Pi_{1}^{*}\left(n_{1}, n_{2}-1, \omega, \alpha\right)\right],
$$

for any $n_{1} \geq 0, n_{2} \geq 1, \mu, \omega>0$, and $\alpha>1$. Since,

$$
\begin{aligned}
\mathbb{E}\left[\Pi_{2}^{*}\left(n_{1}, n_{2}-1, \omega, \alpha\right)\right]= & \left(n_{1}+n_{2}-1\right) \frac{1}{\mu}+\mathbb{E}\left[\Pi^{*}\left(n_{1}, n_{2}-2, \omega, \alpha\right)\right] \\
\leq & \left(n_{1}+n_{2}-1\right) \frac{1}{\mu}+\mathbb{E}\left[\Pi_{1}^{*}\left(n_{1}, n_{2}-2, \omega, \alpha\right)\right] \\
= & \left(n_{1}+n_{2}-1\right) \frac{1}{\mu}+\left(n_{1}+n_{2}-2\right) \frac{\omega}{\alpha-1} \\
& +\int_{0}^{\infty} \mathbb{E}\left[\Pi^{*}\left(n_{1}-1, n_{2}-2, \omega+x, \alpha+1\right)\right] f(x) d x,
\end{aligned}
$$


and

$$
\begin{aligned}
\mathbb{E}\left[\Pi_{1}^{*}\left(n_{1}, n_{2}-1, \omega, \alpha\right)\right]= & \left(n_{1}+n_{2}-1\right) \frac{\omega}{\alpha-1} \\
& +\int_{0}^{\infty} \mathbb{E}\left[\Pi^{*}\left(n_{1}-1, n_{2}-1, \omega+x, \alpha+1\right)\right] f(x) d x,
\end{aligned}
$$

rewriting yields

$$
\begin{aligned}
\frac{n_{1}+n_{2}}{\mu}-\frac{\omega}{\alpha-1} \geq \frac{1}{\mu}+\int_{0}^{\infty}\left(\mathbb{E}\left[\Pi^{*}\left(n_{1}-1, n_{2}-1, \omega+x, \alpha+1\right)\right]\right. \\
\left.-\mathbb{E}\left[\Pi^{*}\left(n_{1}-1, n_{2}-2, \omega+x, \alpha+1\right)\right]\right) f(x) d x \\
\geq \int_{0}^{\infty} \begin{array}{l}
\left(\mathbb{E}\left[\Pi^{*}\left(n_{1}-1, n_{2}, \omega+x, \alpha+1\right)\right]\right. \\
\left.-\mathbb{E}\left[\Pi^{*}\left(n_{1}-1, n_{2}-1, \omega+x, \alpha+1\right)\right]\right) f(x) d x,
\end{array}
\end{aligned}
$$

where the last inequality follows from Lemma 4.6.5. Then,

$$
\begin{aligned}
\mathbb{E}\left[\Pi_{2}^{*}\left(n_{1}, n_{2}, \omega, \alpha\right)\right]= & \left(n_{1}+n_{2}\right) \frac{1}{\mu}+\mathbb{E}\left[\Pi_{1}^{*}\left(n_{1}, n_{2}-1, \omega, \alpha\right)\right] \\
= & \left(n_{1}+n_{2}\right) \frac{1}{\mu}+\left(n_{1}+n_{2}-1\right) \frac{\omega}{\alpha-1} \\
& +\int_{0}^{\infty} \mathbb{E}\left[\Pi^{*}\left(n_{1}-1, n_{2}-1, \omega+x, \alpha+1\right)\right] f(x) d x \\
\geq & \left(n_{1}+n_{2}\right) \frac{\omega}{\alpha-1}+\int_{0}^{\infty} \mathbb{E}\left[\Pi^{*}\left(n_{1}-1, n_{2}, \omega+x, \alpha+1\right)\right] f(x) d x \\
= & \mathbb{E}\left[\Pi_{1}^{*}\left(n_{1}, n_{2}, \omega, \alpha\right)\right],
\end{aligned}
$$

where the inequality follows from (4.14).

It follows immediately from the lemma above that if it is optimal to start processing a job of class $J_{2}$, then all jobs of class will be processed consecutively until none remains.

Theorem 4.6.6. OPT processes all jobs of class $J_{2}$ consecutively.

Proof. Assume that $\mathbb{E}\left[\Pi_{2}^{*}\left(n_{1}, n_{2}, \omega, \alpha\right)\right]<\mathbb{E}\left[\Pi_{1}^{*}\left(n_{1}, n_{2}, \omega, \alpha\right)\right]$ for any $n_{1} \geq 0, n_{2} \geq 1, \mu, \omega>$ 0 , and $\alpha>1$. We want to show that $\mathbb{E}\left[\Pi_{2}^{*}\left(n_{1}, n_{2}-1, \omega, \alpha\right)\right]<\mathbb{E}\left[\Pi_{1}^{*}\left(n_{1}, n_{2}-1, \omega, \alpha\right)\right]$, in which case the theorem follows. By contradiction, assume that $\mathbb{E}\left[\Pi_{2}^{*}\left(n_{1}, n_{2}-1, \omega, \alpha\right)\right] \geq$ $\mathbb{E}\left[\Pi_{1}^{*}\left(n_{1}, n_{2}-1, \omega, \alpha\right)\right]$. Then, $\mathbb{E}\left[\Pi_{2}^{*}\left(n_{1}, n_{2}, \omega, \alpha\right)\right] \geq \mathbb{E}\left[\Pi_{1}^{*}\left(n_{1}, n_{2}, \omega, \alpha\right)\right]$ by Lemma 4.6.5, yielding a contradiction to our original assumption.

We remark that the result given in the theorem above also follows from Theorem 3.4 and Remark 3.5 in the paper of Burnetas and Katehakis [21]. However, our line of reasoning is build upon different arguments and bounds, which provide new insight into the problem at hand. Burnetas and Katehakis introduce a regret function which is the difference between the expected sum of completion times of an optimal policy and a lower bound on this value. They show that instead of minimizing the expected sum of completion times of an optimal policy, one might instead minimize this regret function. Finally, they establish that deciding 
whether it is optimal to process either a job of class $J_{1}$ or a job of class $J_{2}$ does not depend on the number of jobs of class $J_{2}$ which still have to be processed. Hence, all jobs of class $J_{2}$ will be processed consecutively.

Below we show that if OPT starts by processing a job of class $J_{2}$, then the expected processing time of a job of class $J_{1}$ exceeds that of a job of class $J_{2}$.

Theorem 4.6.7. If OPT starts by processing a job of class $J_{2}$, then $\frac{1}{\mu} \leq \frac{\omega}{\alpha-1}$.

Proof. Let $\mathbb{E}\left[\Pi_{2}^{*}\left(n_{1}, n_{2}, \omega, \alpha\right)\right]<\mathbb{E}\left[\Pi_{1}^{*}\left(n_{1}, n_{2}, \omega, \alpha\right)\right]$. From Theorem 4.6.6, we have that in any optimal policy all jobs of class $J_{2}$ are processed consecutively. Hence,

$$
\begin{aligned}
\mathbb{E}\left[\Pi^{*}\left(n_{1}, n_{2}, \omega, \alpha\right)\right] & =\frac{n_{1}\left(n_{1}+1\right)}{2} \frac{\omega}{\alpha-1}+\frac{n_{2}\left(n_{2}+1\right)}{2} \frac{1}{\mu}+n_{1} n_{2} \frac{1}{\mu} \\
& \leq \frac{n_{1}\left(n_{1}+1\right)}{2} \frac{\omega}{\alpha-1}+\frac{n_{2}\left(n_{2}+1\right)}{2} \frac{1}{\mu}+n_{1} n_{2} \frac{\omega}{\alpha-1},
\end{aligned}
$$

where the inequality follows from Lemma 4.6.3. Rewriting gives $\frac{1}{\mu} \leq \frac{\omega}{\alpha-1}$, and the result follows.

From Observation 4.6.2 and Theorem 4.6.6, we have that SEPT, $\ell$-SEPT, and OPT process all jobs of class $J_{2}$ consecutively. In the theorem above, we established that whenever OPT starts processing a job of class $J_{2}$, then so do SEPT and $\ell$-SEPT. Combining these insights yields the following corollary.

Corollary 4.6.8. If OPT starts by processing a job of class $J_{2}$, then SEPT, $\ell-S E P T$, and OPT yield the same schedule.

\subsubsection{Upper bound on the performance guarantees}

We observed that SEPT, $\ell$-SEPT, and OPT yield the same schedule and hence identical objective values for the case that OPT starts by processing a job of class $J_{2}$. In this subsection, we analyze the worst case performance of SEPT and $\ell$-SEPT with respect the performance of OPT in its full generality. For both policies, we provide an upper bound on the performance guarantee depending on the parameters $n_{1}$ and $n_{2}$. Moreover, we show that this upper bound cannot exceed 2.

Theorem 4.6.9. For any $n_{1}, n_{2} \geq 0, \mu, \omega>0$, and $\alpha>1$,

$$
\frac{\mathbb{E}\left[\Pi^{\ell}\left(n_{1}, n_{2}, \omega, \alpha\right)\right]}{\mathbb{E}\left[\Pi^{*}\left(n_{1}, n_{2}, \omega, \alpha\right)\right]} \leq \frac{\mathbb{E}\left[\Pi^{s}\left(n_{1}, n_{2}, \omega, \alpha\right)\right]}{\mathbb{E}\left[\Pi^{*}\left(n_{1}, n_{2}, \omega, \alpha\right)\right]}<\frac{n_{1}^{2}+n_{2}^{2}+2 n_{1} n_{2}+n_{1}+n_{2}}{n_{1}^{2}+n_{2}^{2}+n_{1}+3 n_{2}}<2 .
$$

Proof. The first inequality follows directly from Corollary 4.6.3. In order to prove the second inequality, observe that both policies produce the same schedule and performance in case OPT starts by processing a job of class $J_{2}$, by Corollary 4.6.8. Therefore, we assume that OPT starts by processing a job of class $J_{1}$. By Lemma 4.6 .1 , we then have

$$
\begin{aligned}
\mathbb{E}\left[\Pi_{1}^{*}\left(n_{1}, n_{2}, \omega, \alpha\right)\right] & =\left(n_{1}+n_{2}\right) \frac{\omega}{\alpha-1}+\int_{0}^{\infty} \mathbb{E}\left[\Pi^{*}\left(n_{1}-1, n_{2}, \omega+x, \alpha+1\right)\right] f(x) d x \\
& \geq \frac{n_{1}\left(n_{1}+1\right)}{2} \frac{\omega}{\alpha-1}+\frac{n_{2}\left(n_{2}+1\right)}{2} \frac{1}{\mu}+n_{2} \frac{\omega}{\alpha-1} .
\end{aligned}
$$


Let $n_{\max }=\max \left\{n_{1}, n_{2}\right\}$. Combining (4.15) and Lemma 4.6.3, we obtain

$$
\begin{gathered}
\frac{\mathbb{E}\left[\Pi^{\ell}\left(n_{1}, n_{2}, \omega, \alpha\right)\right]}{\mathbb{E}\left[\Pi^{*}\left(n_{1}, n_{2}, \omega, \alpha\right)\right]} \leq \frac{\frac{\left(n_{1}+1\right) n_{1}}{2} \frac{\omega}{\alpha-1}+\frac{\left(n_{2}+1\right) n_{2}}{2} \frac{1}{\mu}+n_{1} n_{2} \min \left\{\frac{1}{\mu}, \frac{\omega}{\alpha-1}\right\}}{\frac{n_{1}\left(n_{1}+1\right)}{2} \frac{\omega}{\alpha-1}+\frac{n_{2}\left(n_{2}+1\right)}{2} \frac{1}{\mu}+n_{2} \frac{\omega}{\alpha-1}} \\
\leq \frac{\frac{\left(n_{1}+1\right) n_{1}}{2} \frac{\omega}{\alpha-1}+\frac{\left(n_{2}+1\right) n_{2}}{2} \min \left\{\frac{1}{\mu}, \frac{\omega}{\alpha-1}\right\}+n_{1} n_{2} \min \left\{\frac{1}{\mu}, \frac{\omega}{\alpha-1}\right\}}{\frac{\left(n_{1}+1\right) n_{1}}{2} \frac{\omega}{\alpha-1}+\frac{\left(n_{2}+1\right) n_{2}}{2} \min \left\{\frac{1}{\mu}, \frac{\omega}{\alpha-1}\right\}+n_{2} \frac{\omega}{\alpha-1}} \\
\leq \frac{\frac{\left(n_{1}+1\right) n_{1}}{2} \frac{\omega}{\alpha-1}+\frac{\left(n_{2}+1\right) n_{2}}{2} \frac{\omega}{\alpha-1}+n_{1} n_{2} \frac{\omega}{\alpha-1}}{\frac{\left(n_{1}+1\right) n_{1}}{2} \frac{\omega}{\alpha-1}+\frac{\left(n_{2}+1\right) n_{2}}{2} \frac{\omega}{\alpha-1}+n_{2} \frac{\omega}{\alpha-1}} \\
=\frac{n_{1}^{2}+n_{2}^{2}+2 n_{1} n_{2}+n_{1}+n_{2}}{n_{1}^{2}+n_{2}^{2}+n_{1}+3 n_{2}} \leq \frac{4 n_{\max }^{2}+2 n_{\max }}{2 n_{\max }^{2}+4 n_{\max }}<2 .
\end{gathered}
$$

Here, the second inequality follows from the fact that for any constants $a, b, c$ and $d, \frac{a+b}{a+c} \leq \frac{d+b}{d+c}$ in case $b \geq c>0$ and $a \geq d>0$. The third inequality can be seen from the fact that the ratio is increasing in $\frac{1}{\mu}$ for $\frac{1}{\mu} \leq \frac{\omega}{\alpha-1}$. Note that (4.16) and (4.17) cannot both hold with equality simultaneously, hence the strict second inequality in the theorem.

\subsubsection{Tightness of the performance guarantees}

In this subsection, we show that bounds on the performance guarantee of the previous section are tight for SEPT as well as $\ell$-SEPT. By Corollary 4.6.3, it suffices to show that the guarantee of $\ell$-SEPT is tight. In order to do this, we first show that there exists an instance for which the ratio of the performance of $\ell$-SEPT over the performance of OPT approaches the upper bound depending on the number of jobs in each class, arbitrarily close. Again, the intuition behind this instance is identical to the one given in Section 4.3.2 such that SEPT is forced to start with class $J_{1}$, while OPT first needs to process several jobs of the other classes.

Theorem 4.6.10. For any $n_{1}$ and $n_{2}$, there exists parameter settings $\omega, \mu$ and $\alpha>1$, such that

$$
\frac{\mathbb{E}\left[\Pi^{\ell}\left(n_{1}, n_{2}, \omega, \alpha\right)\right]}{\mathbb{E}\left[\Pi^{*}\left(n_{1}, n_{2}, \omega, \alpha\right)\right]}>\frac{n_{1}^{2}+n_{2}^{2}+2 n_{1} n_{2}+n_{1}+n_{2}}{n_{1}^{2}+n_{2}^{2}+n_{1}+3 n_{2}}-\epsilon,
$$

for any $\epsilon>0$.

Proof. First, set $\mu=1$. Then,

$$
\begin{aligned}
\mathbb{E}\left[\Pi^{*}\left(n_{1}, n_{2}, \omega, \alpha\right)\right] \leq & \mathbb{E}\left[\Pi_{1}^{*}\left(n_{1}, n_{2}, \omega, \alpha\right)\right] \\
= & \left(n_{1}+n_{2}\right) \frac{\omega}{\alpha-1}+\int_{0}^{\infty} \mathbb{E}\left[\Pi^{*}\left(n_{1}-1, n_{2}, \omega+x, \alpha+1\right)\right] f(x) d x \\
\leq & \left(n_{1}+n_{2}\right) \frac{\omega}{\alpha-1}+\frac{\left(n_{1}-1\right) n_{1}}{2} \frac{\omega}{\alpha-1}+\frac{n_{2}\left(n_{2}+1\right)}{2} \\
& +\left(n_{1}-1\right) n_{2} \int_{0}^{\infty} \min \left\{1, \frac{\omega+x}{\alpha}\right\} \frac{\alpha \omega^{\alpha}}{(\omega+x)^{\alpha+1}} d x \\
= & n_{2} \frac{\omega}{\alpha-1}+\frac{n_{1}\left(n_{1}+1\right)}{2} \frac{\omega}{\alpha-1}+\frac{n_{2}\left(n_{2}+1\right)}{2} \\
& +\left(n_{1}-1\right) n_{2} \frac{\omega}{\alpha-1}\left[1-\frac{1}{\alpha}\left(\frac{\omega}{\alpha}\right)^{\alpha-1}\right] .
\end{aligned}
$$


Here, the second inequality follows from Lemma 4.6.3, and Proposition 4.2.2.

Next, set $\omega=\alpha-1$. This gives

$$
\mathbb{E}\left[\Pi^{\ell}\left(n_{1}, n_{2}, \alpha-1, \alpha\right)\right]=\frac{n_{1}\left(n_{1}+1\right)}{2}+\frac{n_{2}\left(n_{2}+1\right)}{2}+n_{1} n_{2} .
$$

Combining (4.18) and (4.19), we define

$$
z\left(n_{1}, n_{2}, \alpha\right):=\frac{\frac{n_{1}\left(n_{1}+1\right)}{2}+\frac{n_{2}\left(n_{2}+1\right)}{2}+n_{1} n_{2}}{n_{2}+\frac{n_{1}\left(n_{1}+1\right)}{2}+\frac{n_{2}\left(n_{2}+1\right)}{2}+\left(n_{1}-1\right) n_{2}\left[1-\frac{1}{\alpha}\left(\frac{\alpha-1}{\alpha}\right)^{\alpha-1}\right]} .
$$

Furthermore, define

$$
\rho\left(n_{1}, n_{2}\right):=\frac{n_{1}^{2}+n_{2}^{2}+2 n_{1} n_{2}+n_{1}+n_{2}}{n_{1}^{2}+n_{2}^{2}+n_{1}+3 n_{2}}
$$

Clearly, $z\left(n_{1}, n_{2}, \alpha\right)<\rho\left(n_{1}, n_{2}\right)$, and it follows from Proposition 4.2.1 that

$$
\lim _{\alpha \downarrow 1} z\left(n_{1}, n_{2}, \alpha\right)=\rho\left(n_{1}, n_{2}\right) .
$$

Therefore, for any $\epsilon>0$ there exists an $\tilde{\alpha}>1$ such that

$$
z\left(n_{1}, n_{2}, \tilde{\alpha}\right)>\rho\left(n_{1}, n_{2}\right)-\epsilon .
$$

From equations (4.18) and (4.19), we also have that

$$
\frac{\mathbb{E}\left[\Pi^{\ell}\left(n_{1}, n_{2}, \alpha-1, \alpha\right)\right]}{\mathbb{E}\left[\Pi^{*}\left(n_{1}, n_{2}, \alpha-1, \alpha\right)\right]} \geq z\left(n_{1}, n_{2}, \alpha\right),
$$

for all $n_{1}, n_{2}$ and $\alpha>1$ whenever $\mu=1$ and $\omega=\alpha-1$. Hence, combining Theorem 4.6 .9 and equations (4.20) and (4.21), we find that for any epsilon $\epsilon>0$ there exists an $\tilde{\alpha}>1$, for which

$$
\rho\left(n_{1}, n_{2}\right)>\frac{\mathbb{E}\left[\Pi^{\ell}\left(n_{1}, n_{2}, \tilde{\alpha}-1, \tilde{\alpha}\right)\right]}{\mathbb{E}\left[\Pi^{*}\left(n_{1}, n_{2}, \tilde{\alpha}-1, \tilde{\alpha}\right)\right]}>\rho\left(n_{1}, n_{2}\right)-\epsilon
$$

By letting the number of jobs in both classes of the instance provided in the proof of Theorem 4.6.10 approach infinity, we further obtain the result below.

Corollary 4.6.11. There exist parameters settings $n_{1}, n_{2}, \mu, \omega>0$, and $\alpha>1$ such that

$$
\frac{\mathbb{E}\left[\Pi^{\ell}\left(n_{1}, n_{2}, \omega, \alpha\right)\right]}{\mathbb{E}\left[\Pi^{*}\left(n_{1}, n_{2}, \omega, \alpha\right)\right]}>2-\epsilon
$$

for any $\epsilon>0$.

Proof. Consider the instance in the proof of Theorem 4.6.10. Additionally, set $n_{1}=n_{2}=n$ and define

$$
z(n, \alpha):=\frac{4 n^{2}+2 n}{2 n^{2}+4 n+(n-1) n\left[1-\frac{1}{\alpha}\left(\frac{\alpha-1}{\alpha}\right)^{\alpha-1}\right]}<2 .
$$


Since by Proposition 4.2.1,

$$
\lim _{n \rightarrow \infty} \lim _{\alpha \downarrow 1} z(n, \alpha)=\lim _{n \rightarrow \infty} \frac{4 n^{2}+2 n}{2 n^{2}+4 n}=2,
$$

it follows that for any $\epsilon>0$ there exists values for $\tilde{n}$ and $\tilde{\alpha}$ such that

$$
z(\tilde{n}, \tilde{\alpha})>2-\epsilon
$$

Further, from (4.18), (4.19) and (4.22), there exist values $\tilde{n}$ and $\tilde{\alpha}$ such that

$$
2>\frac{\mathbb{E}\left[\Pi^{*}(\tilde{n}, \tilde{n}, \tilde{\alpha}-1, \tilde{\alpha})\right]}{\mathbb{E}\left[\Pi^{\ell}(\tilde{n}, \tilde{n}, \tilde{\alpha}-1, \tilde{\alpha})\right]} \geq z(\tilde{n}, \tilde{n}, \tilde{\alpha})=z(\tilde{n}, \tilde{\alpha})>2-\epsilon,
$$

for any $\epsilon>0$, and the result follows. Note that $z(\tilde{n}, \tilde{n}, \tilde{\alpha})$ is defined in the proof of Theorem 4.6.10.

Note that this result in combination with Theorem 4.15 leads to a tight constant performance guarantee of 2 for SEPT and $\ell$-SEPT, similar to the bound in Section 4.5.

\subsection{Computational experience}

In this section, we perform a large amount of simulations to compare the average values of SEPT and $\ell$-SEPT with the average optimal Bayesian solution for different job class settings. In order to compute the values of OPT, we used the algorithm presented in Section 4 of the paper of Hamada and Glazebrook [53]. Note that in contrast to Section 4.4, we no longer calculate upper bounds on the expected performance ratio but compare empirical average performance ratios instead. All computations are again performed in MATLAB.

The Bayesian scheduling instances studied are as follows: we consider 2 different problem settings; that is, there are either 2 or 3 different job classes. To study how large the gap between SEPT and $\ell$-SEPT might be, we set the number of jobs in each class equal to 15 . Furthermore, the gamma prior parameters are set such that $\omega_{i}$ and $\left(\alpha_{i}-1\right)$ are both an element of $\{0.5 ; 1.0 ; 5.0 ; 25.0\}$, for each job class $J_{i}(i \in\{1, \ldots, 3\})$. This results in 100 and 400 different computations when there are 2 or 3 job classes, respectively, thereby covering the majority of interesting instances. That is, the cases in which all job classes have either high or low parameter uncertainty, and the mixed case in which some class has high and some other one has low parameter uncertainty. Moreover, these computations could still be performed in a reasonable amount of time. Choosing our settings in a more extreme fashion (like having more jobs in each class) immediately results in difficulties with the precision in calculating OPT. Furthermore, it significantly increases the computation time of this optimal policy, especially in case of 4 or more job classes.

In our computations, 50000 simulations are run to compute the average values of SEPT, $\ell$ SEPT and OPT for each Bayesian scheduling instance. In each of those simulations, we draw for each job class a parameter realization from a gamma distribution. This realization is subsequently used to draw 15 processing time realizations from an exponential distribution. Using these realizations the sum of completion times for each of the policies is calculated. As mentioned before, performance of the policies SEPT and $\ell$-SEPT is measured by the average 
objective value of the policy over the average objective value of OPT. The computational results are presented in Tables 4.2 and 4.3 of Appendix 4.9.4. In the latter table, we supressed for presentational purposes the columns and rows in which all relative deviations of SEPT and $\ell$-SEPT from OPT are smaller than $30 \%$ and $10 \%$, respectively.

A first observation shows that $\ell$-SEPT significantly outperforms SEPT in every setting, regardless whether there are 2 or 3 job classes. When the value of SEPT is far away from OPT, the deviation from $\ell$-SEPT to OPT is at least a factor 3 less than the deviation from SEPT to OPT. On the other hand, when the values of SEPT and OPT are close, then $\ell$-SEPT in most of the cases equals OPT. The performance of $\ell$-SEPT is poorest when one job class, say $J_{i}$, has a low parameter uncertainty $\left(\alpha_{i}-1=\omega_{i}\right.$ and both values large) and all other classes, $J_{j}$ with $j \neq i$, have high parameter uncertainty $\left(\alpha_{j}-1=\omega_{j}\right.$ and both values low). To be more explicit, the worst case for $\ell$-SEPT is obtained whenever $\alpha_{1}-1=\omega_{1}=25$ and $\alpha_{j}-1=\omega_{j}=0.5$ for all other job classes $J_{j}(j \neq 1)$; we find that $\ell$-SEPT is no more than $9.1 \%$ and $10.5 \%$ away from OPT with 2 or 3 job classes. It is interesting to observe that other instances for which $\ell$ SEPT also performs relatively poor usually adhere the same structure, that is, this structure is similar to the one used in constructing our lower bound. Consequently, the computational results again support our conjecture.

The performance of SEPT significantly deteriorates in the presence of more job classes. In the worst setting, we find that SEPT is no more than $32.6 \%$ and $51.0 \%$ away from OPT when having 2 or 3 job classes, respectively. Although SEPT also performs poorly for the instances where $\ell$-SEPT performs relatively bad, the worst case instances are those where $\alpha_{i}=1.5$ and $\omega_{i}=1$ for all job classes $J_{i}$. In order for both $\ell$-SEPT and SEPT to perform bad, we need that all job classes have high parameter uncertainty and almost equal expected processing times of the jobs for the different classes. In that case, the risk is the highest that the two policies start processing the 'wrong' job class. This is indeed the instance for which SEPT performs worst in our computational results. However, since $\ell$-SEPT updates the expected processing time of a class whenever a job of that class has been completed, we should make sure that $\ell$-SEPT does not switch job classes once it started processing the 'wrong' one. Therefore, $\ell$ SEPT needs this job class to have very low parameter uncertainty, which is again confirmed by our computational results.

We remark that when averaging over the 50000 simulations, SEPT has a higher variance than the other two policies. Again this is explained by the fact that SEPT does not update whereas $\ell$-SEPT and OPT might realize sooner or later that they started with the 'wrong' job class and then switch classes.

\subsection{Concluding remarks}

This chapter studies the performance guarantee of two natural extensions of the traditional stochastic scheduling policy SEPT to the setting of Bayesian scheduling. We consider three different problem settings: $m$ job classes with $m$ unknown parameters, 2 job classes with two unknown parameters, and 2 job classes with one known and one unknown parameter. Hamada and Glazebrook [53] formulated a dynamic programming algorithm for these problems, which is not able to handle instances with large number of jobs or with high parameter uncertainty. This calls for the need to develop policies of low computational effort which yield 
good qualitative performance. The policies SEPT and its adaptive variant $\ell$-SEPT are examples of such policies. For the first problem setting, we show that the performance guarantees for both these policies are upper bounded by $m$. We construct instances with non-degenerately distributed processing times showing that this bound of $m$ is tight for SEPT. Moreover, we provide a lower bound of $1+\sqrt{m-1}$ on the performance guarantee of $\ell$-SEPT, and conjecture that this bound is tight. For the last two problem settings, these results are strengthened by giving tight performance guarantees that are strictly less than 2 , and depend fully on the number of jobs in both job classes, $n_{1}$ and $n_{2}$. Finally, we show that $\ell$-SEPT outperforms SEPT in practice by a range of computational results. 


\subsection{Appendix}

\subsubsection{Proofs of Problem Setting I}

Theorem 4.9.1 (Theorem 4.3.7). There exist parameter settings $\boldsymbol{n}>\mathbf{0}, \boldsymbol{\omega}>\mathbf{0}$ and $\boldsymbol{\alpha}>\mathbf{1}$, such that, for any $\epsilon>0$

$$
\frac{\mathbb{E}\left[\Pi^{\ell}(\boldsymbol{n}, \boldsymbol{\omega}, \boldsymbol{\alpha})\right]}{\mathbb{E}\left[\Pi^{*}(\boldsymbol{n}, \boldsymbol{\omega}, \boldsymbol{\alpha})\right]}>1+\sqrt{m-1}-\epsilon
$$

Proof. By Lemma 4.3.5 and Lemma 4.9.2, which is given below, we have that there exist values for $\boldsymbol{\omega}>\mathbf{0}$ and $\boldsymbol{\alpha}>\mathbf{1}$ such that

$$
\frac{\mathbb{E}\left[\Pi^{\ell}(\boldsymbol{n}, \boldsymbol{\omega}, \boldsymbol{\alpha})\right]}{\mathbb{E}\left[\Pi^{*}(\boldsymbol{n}, \boldsymbol{\omega}, \boldsymbol{\alpha})\right]}>\frac{\sum_{i=1}^{m} n_{i}\left(n_{i}+1\right)+2 \sum_{i=2}^{m} n_{1} n_{i}}{\sum_{i=1}^{m} n_{i}\left(n_{i}+1\right)+2 m \sum_{i=1}^{m} n_{i}}-\epsilon
$$

for any $\epsilon>0$. Then, by setting $n_{2}=\ldots=n_{m}=n, n_{1}=n \sqrt{m-1}$ and letting $n$ tend to infinity, we find that for any $\epsilon>0$

$$
\begin{aligned}
\lim _{n \rightarrow \infty} \frac{\mathbb{E}\left[\Pi^{\ell}\left(n_{1}, n_{2}, \boldsymbol{\omega}, \boldsymbol{\alpha}\right)\right]}{\mathbb{E}\left[\Pi^{*}\left(n_{1}, n_{2}, \boldsymbol{\omega}, \boldsymbol{\alpha}\right)\right]} & >\lim _{n \rightarrow \infty} \frac{(m-1)\left(2 n^{2}+n\right)+n \sqrt{m-1}+2(m-1) n^{2} \sqrt{m-1}}{(m-1)\left(2 n^{2}+n\right)+n \sqrt{m-1}+2 m n \sqrt{m-1}+2 m(m-1) n}-\epsilon \\
& =\frac{2(m-1)+2(m-1) \sqrt{m-1})}{2(m-1)}-\epsilon=1+\sqrt{m-1}-\epsilon .
\end{aligned}
$$

Lemma 4.9.2. For any $n \geq 0$, there exist parameter settings $\boldsymbol{\omega}>\mathbf{0}$, and $\boldsymbol{\alpha}>\mathbf{1}$ such that

$$
\frac{\omega_{1}}{\alpha_{1}-1}<\frac{\omega_{2}}{\alpha_{2}-1}=\ldots=\frac{\omega_{m}}{\alpha_{m}-1}=1
$$

and

$$
\mathbb{E}\left[\Pi^{\ell}(\boldsymbol{n}, \boldsymbol{\omega}, \boldsymbol{\alpha})\right]>\sum_{i=1}^{m} \frac{n_{i}\left(n_{i}+1\right)}{2}+\sum_{i=2}^{m} n_{1} n_{i}-\epsilon,
$$

for any $\epsilon>0$.

Proof. For arbitrary $\boldsymbol{\alpha}>1$, set $\omega_{1}=\left(\alpha_{1}-1\right)\left(1-\sqrt{\frac{1}{\alpha_{1}}}\right)$ and $\omega_{2}=\ldots=\omega_{m}=\alpha_{2}-1=\ldots=$ $\alpha_{m}-1$ such that (4.23) is satisfied. Then,

$$
\begin{array}{r}
\mathbb{E}\left[\Pi^{\ell}(\boldsymbol{n}, \boldsymbol{\omega}, \boldsymbol{\alpha})\right]=\frac{\omega_{1}}{\alpha_{1}-1} \sum_{i=1}^{m} n_{i}+\int_{0}^{\infty} \mathbb{E}\left[\Pi^{\ell}\left(\boldsymbol{n}-\boldsymbol{e}_{1}, \boldsymbol{\omega}+x_{1}^{1} \boldsymbol{e}_{1}, \boldsymbol{\alpha}+\boldsymbol{e}_{1}\right)\right] f_{1}^{1}(x) d x_{1}^{1} \\
>\frac{\omega_{1}}{\alpha_{1}-1} \sum_{i=1}^{m} n_{i}+\frac{\left(n_{1}-1\right) n_{1}}{2} \frac{\omega_{1}-1}{\alpha_{1}+n_{1}-2}+\frac{\left(n_{2}-1\right) n_{2}}{2}+\sum_{i=3}^{m} \frac{n_{i}\left(n_{i}+1\right)}{2} \\
+\left(n_{2}+\sum_{i=2}^{m}\left(n_{1}-1\right) n_{i}\right)\left[1-\left(\alpha_{1}+n_{1}-2\right)^{n_{1}-2}\left(\frac{\omega_{1}}{\alpha_{1}}\right)^{\alpha_{1}}\right] \frac{\omega_{1}-1}{\alpha_{1}+n_{1}-2}
\end{array}
$$

where the inequality is due to Lemmas 4.9.4 and 4.9.5. 
Letting $\alpha_{1}$ tend to infinity, we know by (4.24) and Proposition 4.2.4, that

$$
\begin{aligned}
\lim _{\alpha_{1} \rightarrow \infty} \mathbb{E}\left[\Pi^{\ell}(\boldsymbol{n}, \boldsymbol{\omega}, \boldsymbol{\alpha})\right]> & \sum_{i=1}^{m} n_{i}+\frac{\left(n_{1}-1\right) n_{1}}{2}+\frac{\left(n_{2}-1\right) n_{2}}{2} \\
& +\sum_{i=3}^{m} \frac{n_{i}\left(n_{i}+1\right)}{2}+\left(n_{2}+\sum_{i=2}^{m}\left(n_{1}-1\right) n_{i}\right) \\
= & \left(n_{1}+n_{2}\right)+\frac{\left(n_{1}-1\right) n_{1}}{2}+\frac{\left(n_{2}-1\right) n_{2}}{2} \\
& +\sum_{i=3}^{m} \frac{n_{i}\left(n_{i}+1\right)}{2}+\sum_{i=2}^{m} n_{1} n_{i} \\
= & \sum_{i=1}^{m} \frac{n_{i}\left(n_{i}+1\right)}{2}+\sum_{i=2}^{m} n_{1} n_{i} .
\end{aligned}
$$

Hence, for any $\epsilon>0$, there exists an $\alpha^{*}$ such that for all $\alpha_{1}>\alpha^{*}$

$$
\mathbb{E}\left[\Pi^{\ell}\left(n_{1}, n_{2}, \boldsymbol{\omega}, \boldsymbol{\alpha}\right)\right]>\sum_{i=1}^{m} \frac{n_{i}\left(n_{i}+1\right)}{2}+\sum_{i=2}^{m} n_{1} n_{i}-\epsilon,
$$

where $\omega_{1}=\left(\alpha_{1}-1\right)\left(1-\sqrt{\frac{1}{\alpha_{1}}}\right)$ and $\omega_{2}=\ldots=\omega_{m}=\alpha_{2}-1=\ldots=\alpha_{m}-1$.

Lemma 4.9.3. Let $\frac{\omega_{1}}{\alpha_{1}-1}<\frac{\omega_{2}}{\alpha_{2}-1}=\ldots=\frac{\omega_{m}}{\alpha_{m}-1}=1$ and $n_{1}, n_{2} \geq 1$. Then,

$$
\begin{aligned}
\int_{0}^{\infty} \mathbb{E} & {\left[\Pi^{\ell}\left(\boldsymbol{n}, \boldsymbol{\omega}+x_{1}^{1} \boldsymbol{e}_{1}, \boldsymbol{\alpha}+\boldsymbol{e}_{1}\right)\right] f_{1}^{1}\left(x_{1}^{1}\right) d x_{1}^{1}>\frac{\omega_{1}-1}{\alpha_{1}-1} \sum_{i=1}^{m} n_{i} } \\
& +\int_{\alpha_{1}-\omega_{1}}^{\infty}\left[\frac{n_{1}\left(n_{1}+1\right)}{2} \frac{\omega_{1}+x_{1}^{1}}{\alpha_{1}}+\frac{\left(n_{2}-1\right) n_{2}}{2} \frac{\omega_{2}}{\alpha_{2}-1}+\sum_{i=3}^{m} \frac{n_{i}\left(n_{i}+1\right)}{2} \frac{\omega_{i}}{\alpha_{i}-1}\right] f_{1}^{1}\left(x_{1}^{1}\right) d x_{1}^{1} \\
& +\int_{0}^{\alpha_{1}-\omega_{1}}\left[\int_{0}^{\infty} \mathbb{E}\left[\Pi^{\ell}\left(\boldsymbol{n}-\boldsymbol{e}_{1}, \boldsymbol{\omega}+\left(x_{1}^{1}+x_{1}^{2}\right) \boldsymbol{e}_{1}, \boldsymbol{\alpha}+2 \boldsymbol{e}_{1}\right)\right] f_{1}^{2}\left(x_{1}^{2}\right) d x_{1}^{2}\right] f_{1}^{1}\left(x_{1}^{1}\right) d x_{1}^{1} .
\end{aligned}
$$

Proof. Let $\frac{\omega_{1}}{\alpha_{1}-1}<\frac{\omega_{2}}{\alpha_{2}-1}=\ldots=\frac{\omega_{m}}{\alpha_{m}-1}=1$ such that $\omega_{1}<\alpha_{1}-1<\alpha_{1}$ and

$$
\int_{0}^{\infty} \min \left\{\frac{\omega_{1}+x_{1}^{1}}{\alpha_{1}}, 1\right\} f_{1}^{1}\left(x_{1}^{1}\right) d x_{1}^{1} \stackrel{\text { Prop. }}{=} .2 .2 \frac{\omega_{1}}{\alpha_{1}-1}-\frac{1}{\alpha_{1}-1}\left(\frac{\omega_{1}}{\alpha_{1}}\right)^{\alpha_{1}}>\frac{\omega_{1}-1}{\alpha_{1}-1} \text {. }
$$

Then,

$$
\begin{aligned}
& \int_{0}^{\infty} \mathbb{E}\left[\Pi^{\ell}\left(\boldsymbol{n}, \boldsymbol{\omega}+x_{1}^{1} \boldsymbol{e}_{1}, \boldsymbol{\alpha}+\boldsymbol{e}_{1}\right)\right] f_{1}^{1}\left(x_{1}^{1}\right) d x_{1}^{1} \\
& =\int_{0}^{\alpha_{1}-\omega_{1}}\left[\frac{\omega_{1}+x_{1}^{1}}{\alpha_{1}} \sum_{i=1}^{m} n_{i}\right. \\
& \left.\quad+\int_{0}^{\infty} \mathbb{E}\left[\Pi^{\ell}\left(\boldsymbol{n}-\boldsymbol{e}_{1}, \boldsymbol{\omega}+\left(x_{1}^{1}+x_{1}^{2}\right) \boldsymbol{e}_{1}, \boldsymbol{\alpha}+2 \boldsymbol{e}_{1}\right)\right] f_{1}^{2}\left(x_{1}^{2}\right) d x_{1}^{2}\right] f_{1}^{1}\left(x_{1}^{1}\right) d x_{1}^{1} \\
& \quad+\int_{\alpha_{1}-\omega_{1}}^{\infty}\left[\frac{\omega_{2}}{\alpha_{2}-1} \sum_{i=1}^{m} n_{i}\right.
\end{aligned}
$$




$$
\begin{aligned}
& \left.+\int_{0}^{\infty} \mathbb{E}\left[\Pi^{\ell}\left(\boldsymbol{n}-\boldsymbol{e}_{2}, \boldsymbol{\omega}+x_{1}^{1} \boldsymbol{e}_{1}+x_{2}^{1} \boldsymbol{e}_{2}, \boldsymbol{\alpha}+\boldsymbol{e}_{1}+\boldsymbol{e}_{2}\right)\right] f_{2}^{1}\left(x_{2}^{1}\right) d x_{2}^{1}\right] f_{1}^{1}\left(x_{1}^{1}\right) d x_{1}^{1} \\
\underset{>}{\operatorname{Lemma}}+.3 .1 & \left(\sum_{i=1}^{m} n_{i}\right)\left[\int_{0}^{\alpha_{1}-\omega_{1}} \frac{\omega_{1}+x_{1}^{1}}{\alpha_{1}} f_{1}^{1}\left(x_{1}^{1}\right) d x_{1}^{1}+\int_{\alpha_{1}-\omega_{1}}^{\infty} 1 \cdot f_{1}^{1}\left(x_{1}^{1}\right) d x_{1}^{1}\right] \\
& +\int_{0}^{\alpha_{1}-\omega_{1}}\left[\int_{0}^{\infty} \mathbb{E}\left[\Pi^{\ell}\left(\boldsymbol{n}-\boldsymbol{e}_{1}, \boldsymbol{\omega}+\left(x_{1}^{1}+x_{1}^{2}\right) \boldsymbol{e}_{1}, \boldsymbol{\alpha}+2 \boldsymbol{e}_{1}\right)\right] f_{1}^{2}\left(x_{1}^{2}\right) d x_{1}^{2}\right] f_{1}^{1}\left(x_{1}^{1}\right) d x_{1}^{1} \\
& +\int_{\alpha_{1}-\omega_{1}}^{\infty}\left[\frac{n_{1}\left(n_{1}+1\right)}{2} \frac{\omega_{1}+x_{1}^{1}}{\alpha_{1}}+\frac{\left(n_{2}-1\right) n_{2}}{2} \frac{\omega_{2}}{\alpha_{2}-1}+\sum_{i=3}^{m} \frac{n_{i}\left(n_{i}+1\right)}{2} \frac{\omega_{i}}{\alpha_{i}-1}\right] f_{1}^{1}\left(x_{1}^{1}\right) d x_{1}^{1} \\
\stackrel{(4.25)}{>} & \frac{\omega_{1}-1}{\alpha_{1}-1} \sum_{i=1}^{m} n_{i} \\
& +\int_{\alpha_{1}-\omega_{1}}^{\infty}\left[\frac{n_{1}\left(n_{1}+1\right)}{2} \frac{\omega_{1}+x_{1}^{1}}{\alpha_{1}}+\frac{\left(n_{2}-1\right) n_{2}}{2} \frac{\omega_{2}}{\alpha_{2}-1}+\sum_{i=3}^{m} \frac{n_{i}\left(n_{i}+1\right)}{2} \frac{\omega_{i}}{\alpha_{i}-1}\right] f_{1^{1}}\left(x_{1}^{1}\right) d x_{1}^{1} \\
+ & \int_{0}^{\alpha_{1}-\omega_{1}}\left[\int_{0}^{\infty} \mathbb{E}\left[\Pi^{\ell}\left(\boldsymbol{n}-\boldsymbol{e}_{1}, \boldsymbol{\omega}+\left(x_{1}^{1}+x_{1}^{2}\right) \boldsymbol{e}_{1}, \boldsymbol{\alpha}+2 \boldsymbol{e}_{1}\right)\right] f_{1}^{2}\left(x_{1}^{2}\right) d x_{1}^{2}\right] f_{1}^{1}\left(x_{1}^{1}\right) d x_{1}^{1} .
\end{aligned}
$$

Lemma 4.9.4. Let $\frac{\omega_{1}}{\alpha_{1}-1}<\frac{\omega_{2}}{\alpha_{2}-1}=\ldots=\frac{\omega_{m}}{\alpha_{m}-1}=1$ and $n_{1}>0$. Then,

$$
\begin{aligned}
& \int_{0}^{\infty} \mathbb{E}\left[\Pi^{\ell}\left(\boldsymbol{n}, \boldsymbol{\omega}+x_{1}^{1} \boldsymbol{e}_{1}, \boldsymbol{\alpha}+\boldsymbol{e}_{1}\right)\right] f_{1}^{1}\left(x_{1}^{1}\right) d x_{1}^{1} \\
& >\frac{n_{1}\left(n_{1}+1\right)}{2} \frac{\omega_{1}-1}{\alpha_{1}+n_{1}-1}+\frac{\left(n_{2}-1\right) n_{2}}{2}+\sum_{i=3}^{m} \frac{n_{i}\left(n_{i}+1\right)}{2} \\
& +\left(n_{2}+\sum_{i=2}^{m} n_{1} n_{i}\right) \int_{0}^{\alpha_{1}-\omega_{1}} \cdots \int_{0}^{\alpha_{1}+n_{1}-1-\omega_{1}-x_{1}^{1}-\ldots-x_{1}^{n_{1}-1}} \frac{\omega_{1}-1}{\alpha_{1}+n_{1}-1} f_{1}^{n_{1}}\left(x_{1}^{n_{1}}\right) d x_{1}^{n_{1}} \cdots f_{1}^{1}\left(x_{1}^{1}\right) d x_{1}^{1} .
\end{aligned}
$$

Proof. We provide a proof by induction on $n_{1}$. In case $n_{1}=1$, it follows from Lemma 4.9.3 that

$$
\begin{aligned}
& \int_{0}^{\infty} \mathbb{E}\left[\Pi^{\ell}\left(\boldsymbol{n}, \boldsymbol{\omega}+x_{1}^{1} \boldsymbol{e}_{1}, \boldsymbol{\alpha}+\boldsymbol{e}_{1}\right)\right] f_{1}^{1}\left(x_{1}^{1}\right) d x_{1}^{1} \\
> & \left(1+\sum_{i=2}^{m} n_{i}\right) \frac{\omega_{1}-1}{\alpha_{1}-1}+\int_{\alpha_{1}-\omega_{1}}^{\infty}\left[\frac{\omega_{1}+x_{1}^{1}}{\alpha_{1}}+\frac{\left(n_{2}-1\right) n_{2}}{2} \frac{\omega_{2}}{\alpha_{2}-1}+\sum_{i=3}^{m} \frac{n_{i}\left(n_{i}+1\right)}{2} \frac{\omega_{i}}{\alpha_{i}-1}\right] f_{1}^{1}\left(x_{1}^{1}\right) d x_{1}^{1} \\
& +\int_{0}^{\alpha_{1}-\omega_{1}}\left[\int_{0}^{\infty} \mathbb{E}\left[\Pi^{\ell}\left(\boldsymbol{n}-n_{1} \boldsymbol{e}_{1}, \boldsymbol{\omega}+\left(x_{1}^{1}+x_{1}^{2}\right) \boldsymbol{e}_{1}, \boldsymbol{\alpha}+2 \boldsymbol{e}_{1}\right)\right] f_{1}^{2}\left(x_{1}^{2}\right) d x_{1}^{2}\right] f_{1}^{1}\left(x_{1}^{1}\right) d x_{1}^{1} \\
& \underset{>}{\operatorname{Lemma}} 4.3 .1 \\
& +\int_{\alpha_{1}-\omega_{1}}^{\infty} \frac{\omega_{1}+x_{1}^{1}}{\alpha_{1}} f_{1}^{1}\left(x_{1}^{1}\right) d x_{1}^{1}+\int_{0}^{\alpha_{1}-\omega_{1}} n_{2} f_{1}^{1}\left(x_{1}^{1}\right) d x_{1}^{1} \\
> & \frac{\omega_{1}-1}{\alpha_{1}}+\frac{\left(n_{2}-1\right) n_{2}}{2}+\sum_{i=3}^{m} \frac{n_{i}\left(n_{i}+1\right)}{2}+\left(n_{2}+\sum_{i=2}^{m} n_{i}\right) \int_{0}^{\alpha_{1}-\omega_{1}} \frac{\omega_{1}-1}{\alpha_{1}} f_{1}^{1}\left(x_{1}^{1}\right) d x_{1}^{1},
\end{aligned}
$$


where the last inequality is due to $1>\frac{\omega_{1}-1}{\alpha_{1}-1}>\frac{\omega_{1}-1}{\alpha_{1}}$. Now, assume that the lemma statement is correct for $n_{1}$. We will verify that the the statement is also valid for $n_{1}+1$. By Lemma 4.9.3,

$$
\begin{aligned}
& \int_{0}^{\infty} \mathbb{E}\left[\Pi^{\ell}\left(\boldsymbol{n}+\boldsymbol{e}_{1}, \boldsymbol{\omega}+x_{1}^{1} \boldsymbol{e}_{1}, \boldsymbol{\alpha}+\boldsymbol{e}_{1}\right)\right] f_{1}^{1}\left(x_{1}^{1}\right) d x_{1}^{1} \\
& >\left(n_{1}+1+\sum_{i=2}^{m} n_{i}\right) \frac{\omega_{1}-1}{\alpha_{1}-1} \\
& +\int_{\alpha_{1}-\omega_{1}}^{\infty}\left[\frac{\left(n_{1}+1\right)\left(n_{1}+2\right)}{2} \frac{\omega_{1}+x_{1}^{1}}{\alpha_{1}}+\frac{\left(n_{2}-1\right) n_{2}}{2} \frac{\omega_{2}}{\alpha_{2}-1}+\sum_{i=3}^{m} \frac{n_{i}\left(n_{i}+1\right)}{2} \frac{\omega_{i}}{\alpha_{i}-1}\right] f_{1}^{1}\left(x_{1}^{1}\right) d x_{1}^{1} \\
& +\int_{0}^{\alpha_{1}-\omega_{1}}\left[\int_{0}^{\infty} \mathbb{E}\left[\Pi^{\ell}\left(\boldsymbol{n}, \boldsymbol{\omega}+\left(x_{1}^{1}+x_{1}^{2}\right) \boldsymbol{e}_{1}, \boldsymbol{\alpha}+2 \boldsymbol{e}_{1}\right)\right] f_{1}^{2}\left(x_{1}^{2}\right) d x_{1}^{2}\right] f_{1}^{1}\left(x_{1}^{1}\right) d x_{1}^{1} \\
& >\left(n_{1}+1+\sum_{i=2}^{m} n_{i}\right) \frac{\omega_{1}-1}{\alpha_{1}-1} \\
& +\int_{\alpha_{1}-\omega_{1}}^{\infty}\left[\frac{\left(n_{1}+1\right)\left(n_{1}+2\right)}{2} \frac{\omega_{1}+x_{1}^{1}}{\alpha_{1}}+\frac{\left(n_{2}-1\right) n_{2}}{2} \frac{\omega_{2}}{\alpha_{2}-1}+\sum_{i=3}^{m} \frac{n_{i}\left(n_{i}+1\right)}{2} \frac{\omega_{i}}{\alpha_{i}-1}\right] f_{1}^{1}\left(x_{1}^{1}\right) d x_{1}^{1} \\
& +\int_{0}^{\alpha_{1}-\omega_{1}}\left[\frac{n_{1}\left(n_{1}+1\right)}{2} \frac{\omega_{1}+x_{1}^{1}-1}{\alpha_{1}+n_{1}}+\frac{\left(n_{2}-1\right) n_{2}}{2}+\sum_{i=3}^{m} \frac{n_{i}\left(n_{i}+1\right)}{2} \frac{\omega_{i}}{\alpha_{i}-1}\right] f_{1}^{1}\left(x_{1}^{1}\right) d x_{1}^{1} \\
& +\left(n_{2}+\sum_{i=2}^{m} n_{1} n_{i}\right) \int_{0}^{\alpha_{1}-\omega_{1}} \cdots \int_{0}^{\alpha_{1}+n_{1}-\omega_{1}-x_{1}^{1}-\ldots-x_{1}^{n_{1}}} \frac{\omega_{1}+x_{1}^{1}-1}{\alpha_{1}+n_{1}} f_{1}^{n_{1}}\left(x_{1}^{n_{1}+1}\right) d x_{1}^{n_{1}+1} \cdots f_{1}^{1}\left(x_{1}^{1}\right) d x_{1}^{1} \\
& >\left(n_{1}+1+\sum_{i=2}^{m} n_{i}\right) \frac{\omega_{1}-1}{\alpha_{1}+n_{1}}+\frac{n_{2}\left(n_{2}-1\right)}{2}+\sum_{i=3}^{m} \frac{n_{i}\left(n_{i}+1\right)}{2} \\
& +\int_{\alpha_{1}-\omega_{1}}^{\infty}\left[\frac{\left(n_{1}+1\right)\left(n_{1}+2\right)}{2} \frac{\omega_{1}-1}{\alpha_{1}+n_{1}}\right] f_{1}^{1}\left(x_{1}^{1}\right) d x_{1}^{1}+\int_{0}^{\alpha_{1}-\omega_{1}}\left[\frac{n_{1}\left(n_{1}+1\right)}{2} \frac{\omega_{1}-1}{\alpha_{1}+n_{1}}\right] f_{1}^{1}\left(x_{1}^{1}\right) d x_{1}^{1} \\
& +\left(n_{2}+\sum_{i=2}^{m} n_{1} n_{i}\right) \int_{0}^{\alpha_{1}-\omega_{1}} \cdots \int_{0}^{\alpha_{1}+n_{1}-\omega_{1}-x_{1}^{1}-\ldots-x_{1}^{n_{1}}} \frac{\omega_{1}-1}{\alpha_{1}+n_{1}} f_{1}^{n_{1}+1}\left(x_{1}^{n_{1}+1}\right) d x_{1}^{n_{1}+1} \cdots f_{1}^{1}\left(x_{1}^{1}\right) d x_{1}^{1} \\
& >\frac{n_{2}\left(n_{2}-1\right)}{2}+\sum_{i=3}^{m} \frac{n_{i}\left(n_{i}+1\right)}{2}+\frac{\left(n_{1}+1\right)\left(n_{1}+2\right)}{2} \frac{\omega_{1}-1}{\alpha_{1}+n_{1}} \\
& +\left(n_{2}+\sum_{i=2}^{m}\left(n_{1}+1\right) n_{i}\right) \int_{0}^{\alpha_{1}-\omega_{1}} \cdots \int_{0}^{\alpha_{1}+n_{1}-\omega_{1}-x_{1}^{1}-\ldots-x_{1}^{n_{1}}} \frac{\omega_{1}-1}{\alpha_{1}+n_{1}} f_{1}^{n_{1}}\left(x_{1}^{n_{1}+1}\right) d x_{1}^{n_{1}+1} \cdots f_{1}^{1}\left(x_{1}^{1}\right) d x_{1}^{1} \text {, }
\end{aligned}
$$

where the second inequality follows from the induction step. This is allowed, since inside the second integral on the right hand side $x_{1}<\alpha_{1}-\omega_{1}$ such that $\frac{\omega_{1}+x_{1}^{1}}{\alpha_{1}}<1=\frac{\omega_{2}}{\alpha_{2}-1}=\ldots=$ $\frac{\omega_{m}}{\alpha_{m}-1}$.

Lemma 4.9.5. Let $\frac{\omega_{1}}{\alpha_{1}-1}<\frac{\omega_{2}}{\alpha_{2}-1}=\ldots=\frac{\omega_{m}}{\alpha_{m}-1}=1$. Then,

$$
\int_{0}^{\alpha_{1}-\omega_{1}} \cdots \int_{0}^{\alpha_{1}+n_{1}-1-\omega_{1}-x_{1}^{1}-\ldots-x_{1}^{n_{1}-1}} 1 \cdot f_{1}^{n_{1}}\left(x_{1}^{n_{1}}\right) d x_{1}^{n_{1}} \cdots f_{1}^{1}\left(x_{1}^{1}\right) d x_{1}^{1} \geq 1-\left(\alpha_{1}+n_{1}-1\right)^{n_{1}-1}\left(\frac{\omega_{1}}{\alpha_{1}}\right)^{\alpha_{1}}
$$


Proof. We provide a proof by induction on $n_{1}$. In case $n_{1}=1$, we have

$$
\int_{0}^{\alpha_{1}-\omega_{1}} 1 \cdot f_{1}^{1}\left(x_{1}^{1}\right) d x_{1}^{1}=\int_{0}^{\alpha_{1}-\omega_{1}} \frac{\alpha_{1} \cdot \omega_{1}^{\alpha_{1}}}{\left(\omega_{1}+x_{1}^{1}\right)^{\alpha_{1}+1}} d x_{1}^{1}=1-\left(\frac{\omega_{1}}{\alpha_{1}}\right)^{\alpha_{1}} .
$$

Now, assume that the statement is correct for $n_{1}$. We verify it also holds for $n_{1}+1$

$$
\begin{aligned}
\int_{0}^{\alpha_{1}-\omega_{1}} \cdots \int_{0}^{\alpha_{1}+n_{1}-\omega_{1}-x_{1}^{1}-\ldots-x_{1}^{n_{1}}} 1 \cdot f_{1}^{n_{1}+1}\left(x_{1}^{n_{1}+1}\right) d x_{1}^{n_{1}+1} \cdots f_{1}^{1}\left(x_{1}^{1}\right) d x_{1}^{1} \\
\stackrel{(\text { induction })}{\geq} \int_{0}^{\alpha_{1}-\omega_{1}}\left[1-\left(\alpha_{1}+n_{1}\right)^{n_{1}-1}\left(\frac{\omega_{1}+x_{1}^{1}}{\alpha_{1}+1}\right)^{\alpha_{1}+1}\right] f_{1}^{1}\left(x_{1}^{1}\right) d x_{1}^{1} \\
\stackrel{(4.26)}{=} 1-\left(\frac{\omega_{1}}{\alpha_{1}}\right)^{\alpha_{1}}-\int_{0}^{\alpha_{1}-\omega_{1}}\left(\alpha_{1}+n_{1}\right)^{n_{1}-1}\left(\frac{\omega_{1}+x_{1}^{1}}{\alpha_{1}+1}\right)^{\alpha_{1}+1} \frac{\alpha_{1} \omega_{1}^{\alpha_{1}}}{\left(\omega_{1}+x_{1}^{1}\right)^{\alpha_{1}+1}} d x_{1}^{1} \\
=1-\left(\frac{\omega_{1}}{\alpha_{1}}\right)^{\alpha_{1}}-\left(\alpha_{1}+n_{1}\right)^{n_{1}-1} \frac{\alpha_{1}}{\alpha_{1}+1}\left(\frac{\omega_{1}}{\alpha_{1}+1}\right)^{\alpha_{1}}\left(\alpha_{1}-\omega_{1}\right) \\
>1-\left[1+\alpha_{1}\left(\alpha_{1}+n_{1}\right)^{n_{1}-1}\right]\left(\frac{\omega_{1}}{\alpha_{1}}\right)^{\alpha_{1}}>1-\left(\alpha_{1}+n_{1}\right)^{n_{1}}\left(\frac{\omega_{1}}{\alpha_{1}}\right)^{\alpha_{1}} .
\end{aligned}
$$




\subsubsection{Proofs of Tighter bounds for $\ell$-SEPT}

Lemma 4.9.6 (Lemma 4.4.1). For any $\boldsymbol{n} \geq \mathbf{0}, \boldsymbol{\omega}>\mathbf{0}$ and $\boldsymbol{\alpha}>\mathbf{1}$ with $\frac{\omega_{1}}{\alpha_{1}-1} \leq \frac{\omega_{2}}{\alpha_{2}-1} \leq \ldots \leq \frac{\omega_{m}}{\alpha_{m}-1}$,

$$
\mathbb{E}\left[\Pi^{*}(\boldsymbol{n}, \boldsymbol{\omega}, \boldsymbol{\alpha})\right] \geq \sum_{i=1}^{m} \frac{n_{i}\left(n_{i}+1\right)}{2} \frac{\omega_{i}}{\alpha_{i}-1}+\sum_{i=1}^{m-1} \sum_{j=i+1}^{m} n_{i} n_{j} \xi(i, j) \frac{\omega_{i}}{\alpha_{i}-1},
$$

where

$$
\xi(i, j)=1-\frac{\left(\frac{\omega_{i}}{\omega_{i}+\omega_{j}}\right)^{\alpha_{i}-1}\left(\frac{\omega_{j}}{\omega_{i}+\omega_{j}}\right)^{\alpha_{j}-1}}{\left(\alpha_{i}+\alpha_{j}-1\right) \cdot B\left(\alpha_{i}, \alpha_{j}\right)} \in(0,1),
$$

and $B\left(\alpha_{i}, \alpha_{j}\right)=\int_{0}^{1} t^{\alpha_{i}-1}(1-t)^{\alpha_{j}-1} d t$ denotes a Beta function.

Proof. Let $Y=\min \left\{\frac{1}{\Theta_{i}}, \frac{1}{\Theta_{j}}\right\}$ for some $i \neq j$, and let $F_{Y}(\cdot)$ be the corresponding cumulative distribution function. Moreover, let $F_{\Theta_{i}^{-1}}(\cdot)$ denote the cumulative distribution function of random variable $\Theta_{i}^{-1}$, the processing time of jobs in class $i$. Then,

$$
\begin{aligned}
1-F_{Y}(y) & =1-\operatorname{Pr}[Y \leq y]=\operatorname{Pr}\left[\frac{1}{\Theta_{i}}>y\right] \operatorname{Pr}\left[\frac{1}{\Theta_{j}}>y\right]=\left(1-F_{\Theta_{i}^{-1}}(y)\right)\left(1-F_{\Theta_{j}^{-1}}(y)\right) \\
& =\left(1-F_{\Theta_{i}^{-1}}(y)\right)+\left(1-F_{\Theta_{j}^{-1}}(y)\right)-\left(1-F_{\Theta_{i}^{-1}}(y) F_{\Theta_{j}^{-1}}(y)\right)
\end{aligned}
$$

such that

$$
\begin{aligned}
\mathbb{E} & {\left[\min \left\{\frac{1}{\Theta_{i}}, \frac{1}{\Theta_{j}}\right\}\right]=\int_{0}^{\infty}\left(1-F_{Y}(y)\right) d y=\frac{\omega_{i}}{\alpha_{i}-1}+\frac{\omega_{j}}{\alpha_{j}-1}+\int_{0}^{\infty}\left(1-F_{\Theta_{i}^{-1}}(y) F_{\Theta_{j}^{-1}}(y)\right) d y } \\
= & \frac{\omega_{i}}{\alpha_{i}-1}+\frac{\omega_{j}}{\alpha_{j}-1}-\frac{\omega_{i}}{\alpha_{i}-1}\left[\left(\frac{\omega_{i}}{\omega_{j}}\right)^{\alpha_{i}-1} \frac{\Gamma\left(\alpha_{i}+\alpha_{j}-1\right)}{\Gamma\left(\alpha_{i}\right) \Gamma\left(\alpha_{j}\right)}{ }_{2} F_{1}\left(\alpha_{i}+\alpha_{j}-1, \alpha_{i}-1, \alpha_{i},-\left(\frac{\omega_{i}}{\omega_{j}}\right)\right)\right] \\
& -\frac{\omega_{j}}{\alpha_{j}-1}\left[\left(\frac{\omega_{j}}{\omega_{i}}\right)^{\alpha_{j}-1} \frac{\Gamma\left(\alpha_{i}+\alpha_{j}-1\right)}{\Gamma\left(\alpha_{i}\right) \Gamma\left(\alpha_{j}\right)}{ }_{2} F_{1}\left(\alpha_{i}+\alpha_{j}-1, \alpha_{j}-1, \alpha_{j},-\left(\frac{\omega_{j}}{\omega_{i}}\right)\right)\right] \\
= & \frac{\omega_{i}}{\alpha_{i}-1}+\frac{\omega_{j}}{\alpha_{j}-1}-\frac{\omega_{i}}{\alpha_{i}-1} I_{\omega_{i} /\left(\omega_{i}+\omega_{j}\right)}\left(\alpha_{i}-1, \alpha_{j}\right)-\frac{\omega_{j}}{\alpha_{j}-1} I_{\omega_{j} /\left(\omega_{i}+\omega_{j}\right)}\left(\alpha_{j}-1, \alpha_{i}\right),
\end{aligned}
$$

where the third equality follows from solving the integrals, and the last one from the definition of the regularized incomplete beta function

$$
0 \leq I_{x}(a, b)=\frac{B(x ; a, b)}{B(a, b)}=\frac{\int_{0}^{x} t^{a-1}(1-t)^{b-1} d t}{B(a, b)} \leq 1
$$

for all $0 \leq x \leq 1$ and $a, b>0$. Note that

$$
\begin{gathered}
I_{x}(a, b)=I_{x}(a+1, b)+\frac{x^{a}(1-x)^{b}}{a B(a, b)}, \\
I_{x}(a, b)+I_{1-x}(b, a)=1,
\end{gathered}
$$

and

$$
B(a, b)=\frac{\Gamma(a) \Gamma(b)}{\Gamma(a+b)}
$$


By (4.11) and the implied order on the expected processing times of the classes, i.e., $\frac{\omega_{1}}{\alpha_{1}-1} \leq$ $\frac{\omega_{2}}{\alpha_{2}-1} \leq \ldots \leq \frac{\omega_{m}}{\alpha_{m}-1}$, we then have

$$
\begin{aligned}
& \mathbb{E}\left[\Pi^{*}(\boldsymbol{n}, \boldsymbol{\omega}, \boldsymbol{\alpha})\right] \geq \sum_{i=1}^{m} \frac{n_{i}\left(n_{i}+1\right)}{2} \frac{\omega_{i}}{\alpha_{i}-1}+\sum_{i=1}^{m-1} \sum_{j=i+1}^{m} n_{i} n_{j} \mathbb{E}\left[\min \left\{\frac{1}{\Theta_{i}}, \frac{1}{\Theta_{j}}\right\}\right] \\
& \stackrel{(4.27)}{=} \sum_{i=1}^{m} \frac{n_{i}\left(n_{i}+1\right)}{2} \frac{\omega_{i}}{\alpha_{i}-1} \\
& +\sum_{i=1}^{m-1} \sum_{j=i+1}^{m} n_{i} n_{j}\left[\frac{\omega_{i}}{\alpha_{i}-1}+\frac{\omega_{j}}{\alpha_{j}-1}-\frac{\omega_{i}}{\alpha_{i}-1} I_{\omega_{i} /\left(\omega_{i}+\omega_{j}\right)}\left(\alpha_{i}-1, \alpha_{j}\right)-\frac{\omega_{j}}{\alpha_{j}-1} I_{\omega_{j} /\left(\omega_{i}+\omega_{j}\right)}\left(\alpha_{j}-1, \alpha_{i}\right)\right] \\
& \stackrel{(4.28)}{>} \sum_{i=1}^{m} \frac{n_{i}\left(n_{i}+1\right)}{2} \frac{\omega_{i}}{\alpha_{i}-1}+\sum_{i=1}^{m-1} \sum_{j=i+1}^{m} n_{i} n_{j}\left[2-I_{\omega_{i} /\left(\omega_{i}+\omega_{j}\right)}\left(\alpha_{i}-1, \alpha_{j}\right)-I_{\omega_{j} /\left(\omega_{i}+\omega_{j}\right)}\left(\alpha_{j}-1, \alpha_{i}\right)\right] \frac{\omega_{i}}{\alpha_{i}-1} \\
& \stackrel{(4.29)}{=} \sum_{i=1}^{m} \frac{n_{i}\left(n_{i}+1\right)}{2} \frac{\omega_{i}}{\alpha_{i}-1}+\sum_{i=1}^{m-1} \sum_{j=i+1}^{m} n_{i} n_{j}\left[2-\left(I_{\omega_{i} /\left(\omega_{i}+\omega_{j}\right)}\left(\alpha_{i}, \alpha_{j}\right)+\frac{\left(\frac{\omega_{i}}{\omega_{i}+\omega_{j}}\right)^{\alpha_{i}-1}\left(\frac{\omega_{j}}{\omega_{i}+\omega_{j}}\right)^{\alpha_{j}}}{B\left(\alpha_{i}-1, \alpha_{j}\right)\left(\alpha_{i}-1\right)}\right)\right. \\
& \left.-\left(I_{\omega_{j} /\left(\omega_{i}+\omega_{j}\right)}\left(\alpha_{j}, \alpha_{i}\right)+\frac{\left(\frac{\omega_{j}}{\omega_{i}+\omega_{j}}\right)^{\alpha_{j}-1}\left(\frac{\omega_{i}}{\omega_{i}+\omega_{j}}\right)^{\alpha_{i}}}{B\left(\alpha_{j}-1, \alpha_{i}\right)\left(\alpha_{j}-1\right)}\right)\right] \frac{\omega_{i}}{\alpha_{i}-1} .
\end{aligned}
$$

The proof is concluded by rewriting the last line using (4.30), (4.31), and the fact that $\Gamma(a)=$ $(a-1) \Gamma(a-1)$.

Lemma 4.9.7 (Lemma 4.4.2). For any $n \geq 0, \omega>0$ and $\boldsymbol{\alpha}>\mathbf{1}$,

$$
\mathbb{E}\left[\Pi^{\ell}(\boldsymbol{n}, \boldsymbol{\omega}, \boldsymbol{\alpha})\right]=\sum_{i=1}^{m-1} \sum_{j=i+1}^{m} \mathbb{E}\left[\Pi^{\ell}\left(n_{i} \boldsymbol{e}_{i}+n_{j} \boldsymbol{e}_{j}, \boldsymbol{\omega}, \boldsymbol{\alpha}\right)\right]-(m-2) \sum_{i=1}^{m} \mathbb{E}\left[\Pi^{\ell}\left(n_{i} \boldsymbol{e}_{i}, \boldsymbol{\omega}, \boldsymbol{\alpha}\right)\right] .
$$

Proof. We provide a proof by induction. The statement trivially holds if $n=e_{h}$ for any $h \in$ $\{1, \ldots, m\}$,

$$
\begin{aligned}
\mathbb{E}\left[\Pi^{\ell}\left(\boldsymbol{e}_{h}, \boldsymbol{\omega}, \boldsymbol{\alpha}\right)\right] & =\frac{\omega_{h}}{\alpha_{h}-1}=\sum_{i=1}^{m-1} \sum_{j=i+1}^{m} \mathbb{E}\left[\Pi^{\ell}\left(n_{i} \boldsymbol{e}_{i}+n_{j} \boldsymbol{e}_{j}, \boldsymbol{\omega}, \boldsymbol{\alpha}\right)\right]-(m-2) \mathbb{E}\left[\Pi^{\ell}\left(\boldsymbol{e}_{h}, \boldsymbol{\omega}, \boldsymbol{\alpha}\right)\right] \\
& =(m-1) \mathbb{E}\left[\Pi^{\ell}\left(\boldsymbol{e}_{h}, \boldsymbol{\omega}, \boldsymbol{\alpha}\right)\right]-(m-2) \mathbb{E}\left[\Pi^{\ell}\left(\boldsymbol{e}_{h}, \boldsymbol{\omega}, \boldsymbol{\alpha}\right)\right]=\mathbb{E}\left[\Pi^{\ell}\left(\boldsymbol{e}_{h}, \boldsymbol{\omega}, \boldsymbol{\alpha}\right)\right] .
\end{aligned}
$$

Consider two vectors $\boldsymbol{v}, \boldsymbol{w} \in \mathbb{R}^{m}$. We define $\boldsymbol{v} \prec \boldsymbol{w}$ when $v_{i} \leq w_{i}$ for all $i \in\{1, \ldots, m\}$ and there exists some $i \in\{1, \ldots, m\}$ such that $v_{i}<w_{i}$. Now, assume that the statement below is true for all $\boldsymbol{n}^{\prime} \prec \boldsymbol{n}$,

$$
\mathbb{E}\left[\Pi^{\ell}\left(\boldsymbol{n}^{\prime}, \boldsymbol{\omega}, \boldsymbol{\alpha}\right)\right]=\sum_{i=1}^{m-1} \sum_{j=i+1}^{m} \mathbb{E}\left[\Pi^{\ell}\left(n_{i}^{\prime} \boldsymbol{e}_{i}+n_{j}^{\prime} \boldsymbol{e}_{j}, \boldsymbol{\omega}, \boldsymbol{\alpha}\right)\right]-(m-2) \sum_{i=1}^{m} \mathbb{E}\left[\Pi^{\ell}\left(n_{i}^{\prime} \boldsymbol{e}_{i}, \boldsymbol{\omega}, \boldsymbol{\alpha}\right)\right]
$$


We show that (4.32) also holds for $\boldsymbol{n}^{\prime}=\boldsymbol{n}$. W.l.o.g. let $\frac{\omega_{1}}{\alpha_{1}-1}=\min _{i \in\{1, \ldots, m\}} \frac{\omega_{i}}{\alpha_{i}-1}$. Then,

$$
\begin{aligned}
& \mathbb{E}\left[\Pi^{\ell}(\boldsymbol{n}, \boldsymbol{\omega}, \boldsymbol{\alpha})\right]=\frac{\omega_{1}}{\alpha_{1}-1} \sum_{i=1}^{m} n_{i}+\int_{0}^{\infty} \mathbb{E}\left[\Pi^{\ell}\left(\boldsymbol{n}-\boldsymbol{e}_{1}, \boldsymbol{\omega}+x_{1}^{1} \boldsymbol{e}_{1}, \boldsymbol{\alpha}+\boldsymbol{e}_{1}\right)\right] f_{1}^{1}\left(x_{1}^{1}\right) d x_{1}^{1} \\
& \stackrel{(4.32)}{=}\left[\frac{\omega_{1}}{\alpha_{1}-1} \sum_{i=1}^{m} n_{i}\right]+\int_{0}^{\infty}\left(\sum_{j=2}^{m} \mathbb{E}\left[\Pi^{\ell}\left(\left(n_{1}-1\right) \boldsymbol{e}_{1}+n_{j} \boldsymbol{e}_{j}, \boldsymbol{\omega}+x_{1}^{1} \boldsymbol{e}_{1}, \boldsymbol{\alpha}+\boldsymbol{e}_{1}\right)\right]\right) f_{1}^{1}\left(x_{1}^{1}\right) d x_{1}^{1} \\
& +\int_{0}^{\infty}\left(\sum_{i=2}^{m-1} \sum_{j=i+1}^{m} \mathbb{E}\left[\Pi^{\ell}\left(n_{i} \boldsymbol{e}_{i}+n_{j} \boldsymbol{e}_{j}, \boldsymbol{\omega}+x_{1}^{1} \boldsymbol{e}_{1}, \boldsymbol{\alpha}+\boldsymbol{e}_{1}\right)\right]\right) f_{1}^{1}\left(x_{1}^{1}\right) d x_{1}^{1} \\
& -(m-2) \int_{0}^{\infty} \mathbb{E}\left[\Pi^{\ell}\left(\left(n_{1}-1\right) \boldsymbol{e}_{1}, \boldsymbol{\omega}+x_{1}^{1} \boldsymbol{e}_{1}, \boldsymbol{\alpha}+\boldsymbol{e}_{1}\right)\right] f_{1}^{1}\left(x_{1}^{1}\right) d x_{1}^{1} \\
& -(m-2) \int_{0}^{\infty}\left(\sum_{i=2}^{m} \mathbb{E}\left[\Pi^{\ell}\left(n_{i} \boldsymbol{e}_{i}, \boldsymbol{\omega}+x_{1}^{1} \boldsymbol{e}_{1}, \boldsymbol{\alpha}+\boldsymbol{e}_{1}\right)\right]\right) f_{1}^{1}\left(x_{1}^{1}\right) d x_{1}^{1} \\
& =\left((m-2) n_{1}+\sum_{i=1}^{m} n_{i}\right) \frac{\omega_{1}}{\alpha_{1}-1}+\sum_{i=2}^{m-1} \sum_{j=i+1}^{m} \mathbb{E}\left[\Pi^{\ell}\left(n_{i} \boldsymbol{e}_{i}+n_{j} \boldsymbol{e}_{j}, \boldsymbol{\omega}, \boldsymbol{\alpha}\right)\right] \\
& +\int_{0}^{\infty}\left(\sum_{j=2}^{m} \mathbb{E}\left[\Pi^{\ell}\left(\left(n_{1}-1\right) \boldsymbol{e}_{1}+n_{j} \boldsymbol{e}_{j}, \boldsymbol{\omega}+x_{1}^{1} \boldsymbol{e}_{1}, \boldsymbol{\alpha}+\boldsymbol{e}_{1}\right)\right]\right) f_{1}^{1}\left(x_{1}^{1}\right) d x_{1}^{1} \\
& -(m-2) \mathbb{E}\left[\Pi^{\ell}\left(n_{1} \boldsymbol{e}_{1}, \boldsymbol{\omega}, \boldsymbol{\alpha}\right)\right]-(m-2) \sum_{i=2}^{m} \mathbb{E}\left[\Pi^{\ell}\left(n_{i} \boldsymbol{e}_{i}, \boldsymbol{\omega}, \boldsymbol{\alpha}\right)\right] \\
& =\left((m-1) n_{1}+\sum_{i=2}^{m} n_{i}\right) \frac{\omega_{1}}{\alpha_{1}-1}+\sum_{j=2}^{m}\left[\mathbb{E}\left[\Pi^{\ell}\left(n_{1} \boldsymbol{e}_{1}+n_{j} \boldsymbol{e}_{j}, \boldsymbol{\omega}, \boldsymbol{\alpha}\right)\right]-\left(n_{1}+n_{j}\right) \frac{\omega_{1}}{\alpha_{1}-1}\right] \\
& +\sum_{i=2}^{m-1} \sum_{j=i+1}^{m} \mathbb{E}\left[\Pi^{\ell}\left(n_{i} \boldsymbol{e}_{i}+n_{j} \boldsymbol{e}_{j}, \boldsymbol{\omega}, \boldsymbol{\alpha}\right)\right]-(m-2) \sum_{i=1}^{m} \mathbb{E}\left[\Pi^{\ell}\left(n_{i} \boldsymbol{e}_{i}, \boldsymbol{\omega}, \boldsymbol{\alpha}\right)\right] \\
& =\sum_{j=2}^{m} \mathbb{E}\left[\Pi^{\ell}\left(n_{1} \boldsymbol{e}_{1}+n_{j} \boldsymbol{e}_{j}, \boldsymbol{\omega}, \boldsymbol{\alpha}\right)\right]+\sum_{i=2}^{m-1} \sum_{j=i+1}^{m} \mathbb{E}\left[\Pi^{\ell}\left(n_{i} \boldsymbol{e}_{i}+n_{j} \boldsymbol{e}_{j}, \boldsymbol{\omega}, \boldsymbol{\alpha}\right)\right] \\
& -(m-2) \sum_{i=1}^{m} \mathbb{E}\left[\Pi^{\ell}\left(n_{i} \boldsymbol{e}_{i}, \boldsymbol{\omega}, \boldsymbol{\alpha}\right)\right] \\
& =\sum_{i=1}^{m-1} \sum_{j=i+1}^{m} \mathbb{E}\left[\Pi^{\ell}\left(n_{i} \boldsymbol{e}_{i}+n_{j} \boldsymbol{e}_{j}, \boldsymbol{\omega}, \boldsymbol{\alpha}\right)\right]-(m-2) \sum_{i=1}^{m} \mathbb{E}\left[\Pi^{\ell}\left(n_{i} \boldsymbol{e}_{i}, \boldsymbol{\omega}, \boldsymbol{\alpha}\right)\right] .
\end{aligned}
$$

Lemma 4.9.8 (Lemma 4.4.3). For any $\boldsymbol{n} \geq \mathbf{0}, \boldsymbol{\omega}>\mathbf{0}$ and $\boldsymbol{\alpha}>\mathbf{1}$ with $\frac{\omega_{1}}{\alpha_{1}-1} \leq \frac{\omega_{2}}{\alpha_{2}-1} \leq \ldots \leq \frac{\omega_{m}}{\alpha_{m}-1}$, $\mathbb{E}\left[\Pi^{\ell}(\boldsymbol{n}, \boldsymbol{\omega}, \boldsymbol{\alpha})\right] \leq \sum_{i=1}^{m} \frac{n_{i}\left(n_{i}+1\right)}{2} \frac{\omega_{i}}{\alpha_{i}-1}+\sum_{i=1}^{m-1} \sum_{j=i+1}^{m}\left[\left(n_{i}-1\right) n_{j} \frac{\omega_{i}}{\alpha_{i}-1} \zeta(i, j)+n_{j} \frac{\omega_{i}}{\alpha_{i}-1}\right]$, 
where

$$
\zeta(i, j)=\left[1-\frac{1}{\alpha_{i}}\left(\frac{\omega_{i}\left(\alpha_{j}-1\right)}{\alpha_{i} \omega_{j}}\right)^{\alpha_{i}-1}\right] \in(0,1) .
$$

Proof. Let $\boldsymbol{n} \geq \mathbf{0}, \boldsymbol{\omega}>\mathbf{0}$ and $\boldsymbol{\alpha}>\mathbf{1}$ be such that $\frac{\omega_{1}}{\alpha_{1}-1} \leq \frac{\omega_{2}}{\alpha_{2}-1} \leq \ldots \leq \frac{\omega_{m}}{\alpha_{m}-1}$. By Lemma 4.4.2, we then have

$$
\begin{aligned}
\mathbb{E}[ & \left.\Pi^{\ell}(\boldsymbol{n}, \boldsymbol{\omega}, \boldsymbol{\alpha})\right]=\sum_{i=1}^{m-1} \sum_{j=i+1}^{m} \mathbb{E}\left[\Pi^{\ell}\left(n_{i} \boldsymbol{e}_{i}+n_{j} \boldsymbol{e}_{j}, \boldsymbol{\omega}, \boldsymbol{\alpha}\right)\right]-(m-2) \sum_{i=1}^{m} \mathbb{E}\left[\Pi^{\ell}\left(n_{i} \boldsymbol{e}_{i}, \boldsymbol{\omega}, \boldsymbol{\alpha}\right)\right] \\
= & \sum_{i=1}^{m-1} \sum_{j=i+1}^{m}\left[\left(n_{i}+n_{j}\right) \frac{\omega_{i}}{\alpha_{i}-1}+\int_{0}^{\infty} \mathbb{E}\left[\Pi^{\ell}\left(\left(n_{i}-1\right) \boldsymbol{e}_{i}+n_{j} \boldsymbol{e}_{j}, \boldsymbol{\omega}+x_{i}^{1} \boldsymbol{e}_{i}, \boldsymbol{\alpha}+\boldsymbol{e}_{i}\right)\right] f_{i}^{1}\left(x_{i}^{1}\right) d x_{i}^{1}\right] \\
& -(m-2) \sum_{i=1}^{m} \frac{n_{i}\left(n_{i}+1\right)}{2} \frac{\omega_{i}}{\alpha_{i}-1} \\
& \stackrel{(4.7)}{\leq} \sum_{i=1}^{m-1} \sum_{j=i+1}^{m}\left[n_{j} \frac{\omega_{i}}{\alpha_{i}-1}+\frac{n_{i}\left(n_{i}+1\right)}{2} \frac{\omega_{i}}{\alpha_{i}-1}+\frac{n_{j}\left(n_{j}+1\right)}{2} \frac{\omega_{j}}{\alpha_{j}-1}\right. \\
& \left.+\left(n_{i}-1\right) n_{j} \int_{0}^{\infty} \min \left\{\frac{\omega_{i}+x_{i}^{1}}{\alpha_{i}}, \frac{\omega_{j}}{\alpha_{j}-1}\right\} f_{i}^{1}\left(x_{i}^{1}\right) d x_{i}^{1}\right]-(m-2) \sum_{i=1}^{m} \frac{n_{i}\left(n_{i}+1\right)}{2} \frac{\omega_{i}}{\alpha_{i}-1} \\
= & \sum_{i=1}^{m} \frac{n_{i}\left(n_{i}+1\right)}{2} \frac{\omega_{i}}{\alpha_{i}-1}+\sum_{i=1}^{m-1} \sum_{j=i+1}^{m} \frac{\omega_{i}}{\alpha_{i}-1} \\
& +\sum_{i=1}^{m-1} \sum_{j=i+1}^{m}\left(n_{i}-1\right) n_{j} \int_{0}^{\infty} \min \left\{\frac{\omega_{i}+x_{i}^{1}}{\alpha_{i}}, \frac{\omega_{j}}{\alpha_{j}-1}\right\} f_{i}^{1}\left(x_{i}^{1}\right) d x_{i}^{1} \\
\text { Prop. } & 4.4 .4 \\
\quad & \sum_{i=1}^{m} \frac{n_{i}\left(n_{i}+1\right)}{2} \frac{\omega_{i}}{\alpha_{i}-1} \\
& +\sum_{i=1}^{m-1} \sum_{j=i+1}^{m}\left(n_{i}-1\right) n_{j} \frac{\omega_{i}}{\alpha_{i}-1}\left[1-\frac{1}{\alpha_{i}}\left(\frac{\omega_{i}\left(\alpha_{j}-1\right)}{\alpha_{i} \omega_{j}}\right) \alpha^{\alpha_{i}-1}\right]+n_{j} \frac{\omega_{i}}{\alpha_{i}-1} .
\end{aligned}
$$




\subsubsection{Proofs of Problem Setting II}

Theorem 4.9.9 (Theorem 4.5.5). There exist parameter settings $n_{1}, n_{2} \geq 0, \boldsymbol{\omega}>\mathbf{0 ,} \boldsymbol{\alpha}>1$ such that

$$
\frac{\mathbb{E}\left[\Pi^{\ell}\left(n_{1}, n_{2}, \boldsymbol{\omega}, \boldsymbol{\alpha}\right)\right]}{\mathbb{E}\left[\Pi^{*}\left(n_{1}, n_{2}, \boldsymbol{\omega}, \boldsymbol{\alpha}\right)\right]}>\frac{n_{1}^{2}+n_{2}^{2}+2 n_{1} n_{2}+n_{1}+n_{2}}{n_{1}^{2}+n_{2}^{2}+3 n_{1}+n_{2}}-\epsilon
$$

for any $\epsilon>0$.

Proof. The theorem is an immediate consequence of Lemma 4.5.3 and Lemma 4.9.10, which is given below.

Lemma 4.9.10. For any $n_{1}, n_{2} \geq 0$, there exist parameter settings $\boldsymbol{\omega}>\mathbf{0}$, and $\boldsymbol{\alpha}>\mathbf{1}$ such that $\frac{\omega_{1}}{\alpha_{1}-1}<\frac{\omega_{2}}{\alpha_{2}-1}=1$ and

$$
\mathbb{E}\left[\Pi^{\ell}\left(n_{1}, n_{2}, \boldsymbol{\omega}, \boldsymbol{\alpha}\right)\right]>\frac{n_{1}\left(n_{1}+1\right)}{2}+\frac{n_{2}\left(n_{2}+1\right)}{2}+n_{1} n_{2}-\epsilon,
$$

for any $\epsilon>0$.

Proof. For arbitrary $\alpha_{1}>1$, set $\omega_{1}=\left(\alpha_{1}-1\right)\left(1-\sqrt{\frac{1}{\alpha_{1}}}\right)$ such that $\frac{\omega_{1}}{\alpha_{1}-1}=1-\sqrt{\frac{1}{\alpha_{1}}}<1=\frac{\omega_{2}}{\alpha_{2}-1}$. Then,

$$
\begin{aligned}
\mathbb{E}\left[\Pi^{\ell}\left(n_{1}, n_{2}, \boldsymbol{\omega}, \boldsymbol{\alpha}\right)\right]= & \left(n_{1}+n_{2}\right) \frac{\omega_{1}}{\alpha_{1}-1}+\int_{0}^{\infty} \mathbb{E}\left[\Pi^{\ell}\left(n_{1}-1, n_{2}, \boldsymbol{\omega}+x_{1}^{1} \boldsymbol{e}_{1}, \boldsymbol{\alpha}+\boldsymbol{e}_{1}\right)\right] f_{1}^{1}\left(x_{1}^{1}\right) d x_{1}^{1} \\
> & \left(n_{1}+n_{2}\right) \frac{\omega_{1}}{\alpha_{1}-1}+\frac{\left(n_{1}-1\right) n_{1}}{2} \frac{\omega_{1}-1}{\alpha_{1}+n_{1}-2}+\frac{n_{2}\left(n_{2}-1\right)}{2} \\
& +n_{1} n_{2}\left[1-\left(\alpha_{1}+n_{1}-2\right)^{n_{1}-2}\left(\frac{\omega_{1}}{\alpha_{1}}\right)^{\alpha_{1}}\right] \frac{\omega_{1}-1}{\alpha_{1}+n_{1}-2}
\end{aligned}
$$

where the inequality is due to Lemmas 4.9.12 and 4.9.13.

Letting $\alpha_{1}$ tend to infinity, we know by (4.33) and Proposition 4.2.4, that

$$
\begin{aligned}
\lim _{\alpha_{1} \rightarrow \infty} \mathbb{E}\left[\Pi^{\ell}\left(n_{1}, n_{2}, \boldsymbol{\omega}, \boldsymbol{\alpha}\right)\right] & >\left(n_{1}+n_{2}\right)+\frac{\left(n_{1}-1\right) n_{1}}{2}+\frac{n_{2}\left(n_{2}-1\right)}{2}+n_{1} n_{2} \\
& =\frac{n_{1}\left(n_{1}+1\right)}{2}+\frac{n_{2}\left(n_{2}+1\right)}{2}+n_{1} n_{2} .
\end{aligned}
$$

Hence, for any $\epsilon>0$, there exists $\hat{\alpha}$ such that for all $\alpha_{1}>\hat{\alpha}$

$$
\mathbb{E}\left[\Pi^{\ell}\left(n_{1}, n_{2}, \boldsymbol{\omega}, \boldsymbol{\alpha}\right)\right]>\frac{n_{1}\left(n_{1}+1\right)}{2}+\frac{n_{2}\left(n_{2}+1\right)}{2}+n_{1} n_{2}-\epsilon,
$$

where $\omega_{1}=\left(\alpha_{1}-1\right)\left(1-\sqrt{\frac{1}{\alpha_{1}}}\right)$ and $\omega_{2}=\alpha_{2}-1$.

Lemma 4.9.11. Let $\frac{\omega_{1}}{\alpha_{1}-1}<\frac{\omega_{2}}{\alpha_{2}-1}=1$ and $n_{1}, n_{2} \geq 1$. Then,

$$
\begin{aligned}
& \int_{0}^{\infty} \mathbb{E}\left[\Pi^{\ell}\left(n_{1}, n_{2}, \boldsymbol{\omega}+x_{1}^{1} \boldsymbol{e}_{1}, \boldsymbol{\alpha}+\boldsymbol{e}_{1}\right)\right] f_{1}^{1}\left(x_{1}^{1}\right) d x_{1}^{1} \\
& >\left(n_{1}+n_{2}\right) \frac{\omega_{1}-1}{\alpha_{1}-1}+\int_{\alpha_{1}-\omega_{1}}^{\infty}\left[\frac{n_{1}\left(n_{1}+1\right)}{2} \frac{\omega_{1}+x_{1}^{1}}{\alpha_{1}}+\frac{n_{2}\left(n_{2}-1\right)}{2} \frac{\omega_{2}}{\alpha_{2}-1}\right] f_{1}^{1}\left(x_{1}^{1}\right) d x_{1}^{1} \\
& \quad+\int_{0}^{\alpha_{1}-\omega_{1}}\left[\int_{0}^{\infty} \mathbb{E}\left[\Pi^{\ell}\left(n_{1}-1, n_{2}, \boldsymbol{\omega}+\left(x_{1}^{1}+x_{1}^{2}\right) \boldsymbol{e}_{1}, \boldsymbol{\alpha}+2 \boldsymbol{e}_{1}\right)\right] f_{1}^{2}\left(x_{1}^{2}\right) d x_{1}^{2}\right] f_{1}^{1}\left(x_{1}^{1}\right) d x_{1}^{1} .
\end{aligned}
$$


Proof. Let $\frac{\omega_{1}}{\alpha_{1}-1}<\frac{\omega_{2}}{\alpha_{2}-1}=1$ such that $\omega_{1}<\alpha_{1}-1<\alpha_{1}$ and

$$
\int_{0}^{\infty} \min \left\{\frac{\omega_{1}+x_{1}^{1}}{\alpha_{1}} ; 1\right\} f_{1}^{1}\left(x_{1}^{1}\right) d x_{1}^{1}=\frac{\omega_{1}}{\alpha_{1}-1}-\frac{1}{\alpha_{1}-1}\left(\frac{\omega_{1}}{\alpha_{1}}\right)^{\alpha_{1}}>\frac{\omega_{1}-1}{\alpha_{1}-1}
$$

Then,

$$
\begin{aligned}
& \int_{0}^{\infty} \mathbb{E}\left[\Pi^{\ell}\left(n_{1}, n_{2}, \boldsymbol{\omega}+x_{1}^{1} \boldsymbol{e}_{1}, \boldsymbol{\alpha}+\boldsymbol{e}_{1}\right)\right] f_{1}^{1}\left(x_{1}^{1}\right) d x_{1}^{1} \\
& =\int_{0}^{\alpha_{1}-\omega_{1}}\left[\left(n_{1}+n_{2}\right) \frac{\omega_{1}+x_{1}^{1}}{\alpha_{1}}\right. \\
& \left.+\int_{0}^{\infty} \mathbb{E}\left[\Pi^{\ell}\left(n_{1}-1, n_{2}, \boldsymbol{\omega}+\left(x_{1}^{1}+x_{1}^{2}\right) \boldsymbol{e}_{1}, \boldsymbol{\alpha}+2 \boldsymbol{e}_{1}\right)\right] f_{1}^{2}\left(x_{1}^{2}\right) d x_{1}^{2}\right] f_{1}^{1}\left(x_{1}^{1}\right) d x_{1}^{1} \\
& +\int_{\alpha_{1}-\omega_{1}}^{\infty}\left[\left(n_{1}+n_{2}\right) \frac{\omega_{2}}{\alpha_{2}-1}\right. \\
& \left.+\int_{0}^{\infty} \mathbb{E}\left[\Pi^{\ell}\left(n_{1}, n_{2}-1, \boldsymbol{\omega}+x_{1}^{1} \boldsymbol{e}_{1}+x_{2}^{1} \boldsymbol{e}_{2}, \boldsymbol{\alpha}+\boldsymbol{e}_{1}+\boldsymbol{e}_{2}\right)\right] f_{2}^{1}\left(x_{2}^{1}\right) d x_{2}^{1}\right] f_{1}^{1}\left(x_{1}^{1}\right) d x_{1}^{1} \\
& \stackrel{\text { Lemma }}{>}{ }^{4.3 .1}\left(n_{1}+n_{2}\right)\left[\int_{0}^{\alpha_{1}-\omega_{1}} \frac{\omega_{1}+x_{1}^{1}}{\alpha_{1}} f_{1}^{1}\left(x_{1}^{1}\right) d x_{1}^{1}+\int_{\alpha_{1}-\omega_{1}}^{\infty} 1 \cdot f_{1}^{1}\left(x_{1}^{1}\right) d x_{1}^{1}\right] \\
& +\int_{0}^{\alpha_{1}-\omega_{1}}\left[\int_{0}^{\infty} \mathbb{E}\left[\Pi^{\ell}\left(n_{1}-1, n_{2}, \boldsymbol{\omega}+\left(x_{1}^{1}+x_{1}^{2}\right) \boldsymbol{e}_{1}, \boldsymbol{\alpha}+2 \boldsymbol{e}_{1}\right)\right] f_{1}^{2}\left(x_{1}^{2}\right) d x_{1}^{2}\right] f_{1}^{1}\left(x_{1}^{1}\right) d x_{1}^{1} \\
& +\int_{\alpha_{1}-\omega_{1}}^{\infty}\left[\frac{n_{1}\left(n_{1}+1\right)}{2} \frac{\omega_{1}+x_{1}^{1}}{\alpha_{1}}+\frac{n_{2}\left(n_{2}-1\right)}{2} \frac{\omega_{2}}{\alpha_{2}-1}\right] f_{1}^{1}\left(x_{1}^{1}\right) d x_{1}^{1} \\
& \stackrel{(4.34)}{>}\left(n_{1}+n_{2}\right) \frac{\omega_{1}-1}{\alpha_{1}-1}+\int_{\alpha_{1}-\omega_{1}}^{\infty}\left[\frac{n_{1}\left(n_{1}+1\right)}{2} \frac{\omega_{1}+x_{1}^{1}}{\alpha_{1}}+\frac{n_{2}\left(n_{2}-1\right)}{2} \frac{\omega_{2}}{\alpha_{2}-1}\right] f_{1}^{1}\left(x_{1}^{1}\right) d x_{1}^{1} \\
& +\int_{0}^{\alpha_{1}-\omega_{1}}\left[\int_{0}^{\infty} \mathbb{E}\left[\Pi^{\ell}\left(n_{1}-1, n_{2}, \boldsymbol{\omega}+\left(x_{1}^{1}+x_{1}^{2}\right) \boldsymbol{e}_{1}, \boldsymbol{\alpha}+2 \boldsymbol{e}_{1}\right)\right] f_{1}^{2}\left(x_{1}^{2}\right) d x_{1}^{2}\right] f_{1}^{1}\left(x_{1}^{1}\right) d x_{1}^{1} .
\end{aligned}
$$

Lemma 4.9.12. Let $\frac{\omega_{1}}{\alpha_{1}-1}<\frac{\omega_{2}}{\alpha_{2}-1}=1$ and $n_{1}>0$. Then,

$$
\begin{aligned}
\int_{0}^{\infty} & \mathbb{E}\left[\Pi^{\ell}\left(n_{1}, n_{2}, \boldsymbol{\omega}+x_{1}^{1} \boldsymbol{e}_{1}, \boldsymbol{\alpha}+\boldsymbol{e}_{1}\right)\right] f_{1}^{1}\left(x_{1}^{1}\right) d x_{1}^{1}>\frac{n_{1}\left(n_{1}+1\right)}{2} \frac{\omega_{1}-1}{\alpha_{1}+n_{1}-1}+\frac{n_{2}\left(n_{2}-1\right)}{2} \\
& +\left(n_{1}+1\right) n_{2} \int_{0}^{\alpha_{1}-\omega_{1}} \cdots \int_{0}^{\alpha_{1}+n_{1}-1-\omega_{1}-x_{1}^{1}-\ldots-x_{1}^{n_{1}-1}} \frac{\omega_{1}-1}{\alpha_{1}+n_{1}-1} f_{1}^{n_{1}}\left(x_{1}^{n_{1}}\right) d x_{1}^{n_{1}} \cdots f_{1}^{1}\left(x_{1}^{1}\right) d x_{1}^{1} .
\end{aligned}
$$

Proof. We provide a proof by induction on $n_{1}$. In case $n_{1}=1$, it follows from Lemma 4.9.11 
that

$$
\begin{aligned}
& \int_{0}^{\infty} \mathbb{E}\left[\Pi^{\ell}\left(1, n_{2}, \boldsymbol{\omega}+x_{1}^{1} \boldsymbol{e}_{1}, \boldsymbol{\alpha}+\boldsymbol{e}_{1}\right)\right] f_{1}^{1}\left(x_{1}^{1}\right) d x_{1}^{1} \\
&>\left(1+n_{2}\right) \frac{\omega_{1}-1}{\alpha_{1}-1}+\int_{\alpha_{1}-\omega_{1}}^{\infty}\left[\frac{\omega_{1}+x_{1}^{1}}{\alpha_{1}}+\frac{n_{2}\left(n_{2}-1\right)}{2} \frac{\omega_{2}}{\alpha_{2}-1}\right] f_{1}^{1}\left(x_{1}^{1}\right) d x_{1}^{1} \\
&+\int_{0}^{\alpha_{1}-\omega_{1}}\left[\int_{0}^{\infty} \mathbb{E}\left[\Pi^{\ell}\left(0, n_{2}, \boldsymbol{\omega}+\left(x_{1}^{1}+x_{1}^{2}\right) \boldsymbol{e}_{1}, \boldsymbol{\alpha}+2 \boldsymbol{e}_{1}\right)\right] f_{1}^{2}\left(x_{1}^{2}\right) d x_{1}^{2}\right] f_{1}^{1}\left(x_{1}^{1}\right) d x_{1}^{1} \\
&=\left(1+n_{2}\right) \frac{\omega_{1}-1}{\alpha_{1}-1}+\frac{n_{2}\left(n_{2}-1\right)}{2}+\int_{\alpha_{1}-\omega_{1}}^{\infty} \frac{\omega_{1}+x_{1}^{1}}{\alpha_{1}} f_{1}^{1}\left(x_{1}^{1}\right) d x_{1}^{1}+\int_{0}^{\alpha_{1}-\omega_{1}} n_{2} f_{1}^{1}\left(x_{1}^{1}\right) d x_{1}^{1} \\
&> \frac{\omega_{1}-1}{\alpha_{1}}+\frac{n_{2}\left(n_{2}-1\right)}{2}+2 n_{2} \int_{0}^{\alpha_{1}-\omega_{1}} \frac{\omega_{1}-1}{\alpha_{1}} f_{1}^{1}\left(x_{1}^{1}\right) d x_{1}^{1},
\end{aligned}
$$

where the last inequality is due to $1>\frac{\omega_{1}-1}{\alpha_{1}-1}>\frac{\omega_{1}-1}{\alpha_{1}}$. Now, assume that the lemma statement is correct for $n_{1}$. We will verify that the the statement is also valid for $n_{1}+1$. By Lemma 4.9.11,

$$
\begin{aligned}
\int_{0}^{\infty} & \mathbb{E}\left[\Pi^{\ell}\left(n_{1}+1, n_{2}, \boldsymbol{\omega}+x_{1}^{1} \boldsymbol{e}_{1}, \boldsymbol{\alpha}+\boldsymbol{e}_{1}\right)\right] f_{1}^{1}\left(x_{1}^{1}\right) d x_{1}^{1} \\
> & \left(n_{1}+1+n_{2}\right) \frac{\omega_{1}-1}{\alpha_{1}-1}+\int_{\alpha_{1}-\omega_{1}}^{\infty}\left[\frac{\left(n_{1}+1\right)\left(n_{1}+2\right)}{2} \frac{\omega_{1}+x_{1}^{1}}{\alpha_{1}}+\frac{n_{2}\left(n_{2}-1\right)}{2} \frac{\omega_{2}}{\alpha_{2}-1}\right] f_{1}^{1}\left(x_{1}^{1}\right) d x_{1}^{1} \\
& +\int_{0}^{\alpha_{1}-\omega_{1}}\left[\int_{0}^{\infty} \mathbb{E}\left[\Pi^{\ell}\left(n_{1}, n_{2}, \boldsymbol{\omega}+\left(x_{1}^{1}+x_{1}^{2}\right) \boldsymbol{e}_{1}, \boldsymbol{\alpha}+2 \boldsymbol{e}_{1}\right)\right] f_{1}^{2}\left(x_{1}^{2}\right) d x_{1}^{2}\right] f_{1}^{1}\left(x_{1}^{1}\right) d x_{1}^{1} \\
> & \left(n_{1}+1+n_{2}\right) \frac{\omega_{1}-1}{\alpha_{1}-1}+\int_{\alpha_{1}-\omega_{1}}^{\infty}\left[\frac{\left(n_{1}+1\right)\left(n_{1}+2\right)}{2} \frac{\omega_{1}+x_{1}^{1}}{\alpha_{1}}+\frac{n_{2}\left(n_{2}-1\right)}{2} \frac{\omega_{2}}{\alpha_{2}-1}\right] f_{1}^{1}\left(x_{1}^{1}\right) d x_{1}^{1} \\
& +\int_{0}^{\alpha_{1}-\omega_{1}}\left[\frac{n_{1}\left(n_{1}+1\right)}{2} \frac{\omega_{1}+x_{1}^{1}-1}{\alpha_{1}+n_{1}}+\frac{n_{2}\left(n_{2}-1\right)}{2}\right] f_{1}^{1}\left(x_{1}^{1}\right) d x_{1}^{1} \\
& +\left(n_{1}+1\right) n_{2} \int_{0}^{\alpha_{1}-\omega_{1}} \cdots \int_{0}^{\alpha_{1}+n_{1}-\omega_{1}-x_{1}^{1}-\ldots-x_{1}^{n_{1}}} \frac{\omega_{1}+x_{1}^{1}-1}{\alpha_{1}} f_{1}^{n_{1}}\left(x_{1}^{n_{1}+1}\right) d x_{1}^{n_{1}+1} \cdots f_{1}^{1}\left(x_{1}^{1}\right) d x_{1}^{1} \\
> & \left(n_{1}+1+n_{2}\right) \frac{\omega_{1}-1}{\alpha_{1}+n_{1}}+\frac{n_{2}\left(n_{2}-1\right)}{2} \\
& \left.+\int_{\alpha_{1}-\omega_{1}}^{\infty} \frac{\left(n_{1}+1\right)\left(n_{1}+2\right)}{2} \frac{\omega_{1}-1}{\alpha_{1}+n_{1}}\right] f_{1}^{1}\left(x_{1}^{1}\right) d x_{1}^{1}+\int_{0}^{\alpha_{1}-\omega_{1}}\left[\frac{n_{1}\left(n_{1}+1\right)}{2} \frac{\omega_{1}-1}{\alpha_{1}+n_{1}}\right] f_{1}^{1}\left(x_{1}^{1}\right) d x_{1}^{1} \\
& +\left(n_{1}+1\right) n_{2} \int_{0}^{\alpha_{1}-\omega_{1}} \cdots \int_{0}^{\alpha_{1}+n_{1}-\omega_{1}-x_{1}^{1}-\ldots-x_{1}^{n_{1}}} \frac{\omega_{1}-1}{\alpha_{1}+n_{1}} f_{1}^{n_{1}+1}\left(x_{1}^{n_{1}+1}\right) d x_{1}^{n_{1}+1} \ldots f_{1}^{1}\left(x_{1}^{1}\right) d x_{1}^{1} \\
> & \frac{n_{2}\left(n_{2}-1\right)}{2}+\frac{\left(n_{1}+1\right)\left(n_{1}+2\right)}{2} \frac{\omega_{1}-1}{\alpha_{1}+n_{1}} \\
& +\left(n_{1}+2\right) n_{2} \int_{0}^{\alpha_{1}-\omega_{1}} \cdots \int_{0}^{\alpha_{1}+n_{1}-\omega_{1}-x_{1}^{1}-\ldots-x_{1}^{n_{1}}} \frac{\omega_{1}-1}{\alpha_{1}+n_{1}} f_{1}^{n_{1}}\left(x_{1}^{n_{1}+1}\right) d x_{1}^{n_{1}+1} \cdots f_{1}^{1}\left(x_{1}^{1}\right) d x_{1}^{1},
\end{aligned}
$$

where the second inequality follows from the induction step. This is allowed, since inside the second integral on the right hand side $x_{1}<\alpha_{1}-\omega_{1}$ such that $\frac{\omega_{1}+x_{1}^{1}}{\alpha_{1}}<1=\frac{\omega_{2}}{\alpha_{2}-1}$.

Lemma 4.9.13. Let $\frac{\omega_{1}}{\alpha_{1}-1}<\frac{\omega_{2}}{\alpha_{2}-1}=1$. Then,

$$
\int_{0}^{\alpha_{1}-\omega_{1}} \cdots \int_{0}^{\alpha_{1}+n_{1}-1-\omega_{1}-x_{1}^{1}-\ldots-x_{1}^{n_{1}-1}} 1 \cdot f_{1}^{n_{1}}\left(x_{1}^{n_{1}}\right) d x_{1}^{n_{1}} \cdots f_{1}^{1}\left(x_{1}^{1}\right) d x_{1}^{1} \geq 1-\left(\alpha_{1}+n_{1}-1\right)^{n_{1}-1}\left(\frac{\omega_{1}}{\alpha_{1}}\right)^{\alpha_{1}}
$$


Proof. We provide a proof by induction on $n_{1}$. In case $n_{1}=1$, we have

$$
\int_{0}^{\alpha_{1}-\omega_{1}} 1 \cdot f_{1}^{1}\left(x_{1}^{1}\right) d x_{1}^{1}=\int_{0}^{\alpha_{1}-\omega_{1}} \frac{\alpha_{1} \cdot \omega_{1}^{\alpha_{1}}}{\left(\omega_{1}+x_{1}^{1}\right)^{\alpha_{1}+1}} d x_{1}^{1}=1-\left(\frac{\omega_{1}}{\alpha_{1}}\right)^{\alpha_{1}} .
$$

Now, assume that the statement is correct for $n_{1}$. We verify it also holds for $n_{1}+1$

$$
\begin{aligned}
\int_{0}^{\alpha_{1}-\omega_{1}} \cdots \int_{0}^{\alpha_{1}+n_{1}-\omega_{1}-x_{1}^{1}-\ldots-x_{1}^{n_{1}}} 1 \cdot f_{1}^{n_{1}+1}\left(x_{1}^{n_{1}+1}\right) d x_{1}^{n_{1}+1} \cdots f_{1}^{1}\left(x_{1}^{1}\right) d x_{1}^{1} \\
\stackrel{\text { (induction) }}{\geq} \int_{0}^{\alpha_{1}-\omega_{1}}\left[1-\left(\alpha_{1}+n_{1}\right)^{n_{1}-1}\left(\frac{\omega_{1}+x_{1}^{1}}{\alpha_{1}+1}\right)^{\alpha_{1}+1}\right] f_{1}^{1}\left(x_{1}^{1}\right) d x_{1}^{1} \\
\stackrel{(4.35)}{=} 1-\left(\frac{\omega_{1}}{\alpha_{1}}\right)^{\alpha_{1}}-\int_{0}^{\alpha_{1}-\omega_{1}}\left(\alpha_{1}+n_{1}\right)^{n_{1}-1}\left(\frac{\omega_{1}+x_{1}^{1}}{\alpha_{1}+1}\right)^{\alpha_{1}+1} \frac{\alpha_{1} \omega_{1}^{\alpha_{1}}}{\left(\omega_{1}+x_{1}^{1}\right)^{\alpha_{1}+1}} d x_{1}^{1} \\
=1-\left(\frac{\omega_{1}}{\alpha_{1}}\right)^{\alpha_{1}}-\left(\alpha_{1}+n_{1}\right)^{n_{1}-1} \frac{\alpha_{1}}{\alpha_{1}+1}\left(\frac{\omega_{1}}{\alpha_{1}+1}\right)^{\alpha_{1}}\left(\alpha_{1}-\omega_{1}\right) \\
>1-\left[1+\alpha_{1}\left(\alpha_{1}+n_{1}\right)^{n_{1}-1}\right]\left(\frac{\omega_{1}}{\alpha_{1}}\right)^{\alpha_{1}}>1-\left(\alpha_{1}+n_{1}\right)^{n_{1}}\left(\frac{\omega_{1}}{\alpha_{1}}\right)^{\alpha_{1}} .
\end{aligned}
$$




\subsubsection{Tables of computational results}

\begin{tabular}{|cc|cccccccccc|}
\hline & $\alpha_{1}$ & 1.5 & 1.5 & 1.5 & 1.5 & 2 & 2 & 2 & 6 & 6 & 26 \\
& $\alpha_{2}$ & 1.5 & 2 & 6 & 26 & 2 & 6 & 26 & 6 & 26 & 26 \\
$\omega_{1}$ & $\omega_{2}$ & & & & & & & & & & \\
\hline 0.5 & 0.5 & 32.1 & 12.2 & & & 23.5 & & & 7.4 & & 1.9 \\
& & 1.7 & 1.7 & & & 1.1 & & & 0.2 & & \\
\hline 0.5 & 1 & 18.6 & 29.8 & 0.2 & & 9.1 & 1.1 & & 0.5 & & \\
& & 1.4 & 3.9 & 0.1 & & 0.7 & 0.7 & & 0.1 & & \\
\hline 0.5 & 5 & 2.3 & 5.8 & 26.2 & & 0.7 & 6.9 & & & 0.4 & \\
& & 0.3 & 0.2 & 7.8 & & 0.1 & 0.2 & & & & \\
\hline 0.5 & 25 & 0.3 & 1.1 & 5.0 & 25.0 & & 0.6 & 6.7 & & & \\
& & & & & 9.1 & & & 0.3 & & & \\
\hline 1 & 1 & 32.6 & 12.0 & & & 22.3 & 0.1 & & 7.5 & & 2.0 \\
& & 1.8 & 2.0 & & & 0.9 & & & 0.2 & & \\
\hline 1 & 5 & 6.7 & 13.4 & 5.3 & & 2.0 & 18.1 & & & 5.7 & \\
& & 0.5 & 0.3 & 4.1 & & 0.2 & 4.6 & & & 1.8 & \\
\hline 1 & 25 & 0.6 & 1.7 & 10.6 & 5.1 & 0.1 & 1.5 & 17.5 & & & \\
& & & 0.1 & 0.1 & 5.1 & & & 6.4 & & & \\
\hline 5 & 5 & 32.3 & 12.6 & & & 23.5 & & & 7.4 & & 1.9 \\
& & 1.9 & 1.9 & & & 1.1 & & & 0.2 & & \\
\hline 5 & 25 & 5.9 & 11.0 & 5.8 & & 2.5 & 17.9 & & & 6.0 & \\
& & 0.7 & 0.4 & 4.4 & & 0.2 & 4.4 & & & 1.8 & \\
\hline 25 & 25 & 30.6 & 12.8 & & & 23.0 & & & 7.3 & & 2.0 \\
& & 2.2 & 2.1 & & & 1.1 & & & 0.2 & & \\
\hline
\end{tabular}

Table 4.2: Relative deviation of SEPT and $\ell$-SEPT from OPT in percentages, with 2 job classes. The upper value in a cell is the deviation for SEPT, the lower one is the deviation for $\ell$-SEPT. In case the deviation from either SEPT or $\ell$-SEPT from the optimal policy is less than $0.1 \%$, the value has been surpressed in the table. 


\begin{tabular}{|c|c|c|c|c|c|c|c|c|c|c|c|c|c|c|}
\hline & & $\alpha_{1}$ & 1.5 & 1.5 & 1.5 & 1.5 & 1.5 & 1.5 & 1.5 & 1.5 & 1.5 & 1.5 & 2 & 2 \\
\hline & & $\alpha_{2}$ & 1.5 & 1.5 & 1.5 & 1.5 & 2 & 2 & 2 & 6 & 6 & 26 & 2 & 2 \\
\hline & & $\alpha_{3}$ & 1.5 & 2 & 6 & 26 & 2 & 6 & 26 & 6 & 26 & 26 & 2 & 26 \\
\hline$\omega_{1}$ & $\omega_{2}$ & $\omega_{3}$ & & & & & & & & & & & & \\
\hline 0.5 & 0.5 & 0.5 & 48.7 & 30.0 & 28.8 & 33.6 & 23.0 & 9.6 & 12.6 & 1.6 & & 0.1 & 33.0 & 21.6 \\
\hline & & & 2.7 & 2.9 & 1.4 & 1.5 & 2.4 & 1.5 & 1.8 & & & & 1.5 & 1.0 \\
\hline 0.5 & 0.5 & 1 & 36.1 & 48.8 & 24.3 & 30.7 & 28.9 & 9.6 & 11.4 & 0.2 & & & 20.1 & 19.9 \\
\hline & & & 2.1 & 4.7 & 1.4 & 1.5 & 3.5 & 1.5 & 1.7 & 0.1 & & & 1.2 & 0.9 \\
\hline 0.5 & 0.5 & 5 & 10.7 & 16.9 & 40.5 & 26.2 & 7.6 & 23.3 & 8.8 & 22.4 & 0.1 & & 5.6 & 14.2 \\
\hline & & & 0.6 & 0.7 & 8.8 & 1.3 & 0.6 & 5.6 & 1.3 & 6.7 & & & 0.3 & 0.7 \\
\hline 0.5 & 0.5 & 25 & 2.6 & 4.2 & 15.6 & 42.2 & 1.9 & 7.2 & 23.3 & 3.6 & 20.9 & 24.1 & 1.4 & 16.3 \\
\hline & & & 0.2 & 0.2 & 0.6 & 10.5 & 0.1 & 0.5 & 7.0 & 0.1 & 8.5 & 9.2 & 0.1 & 0.7 \\
\hline 0.5 & 1 & 1 & 36.2 & 27.3 & 15.0 & 17.5 & 39.7 & 22.9 & 30.4 & 2.4 & 0.1 & 0.2 & 22.2 & 9.2 \\
\hline & & & 2.3 & 3.1 & 0.9 & 1.3 & 4.5 & 3.1 & 3.6 & 0.3 & 0.1 & & 1.3 & 0.6 \\
\hline 0.5 & 1 & 5 & 10.1 & 17.5 & 22.6 & 15.5 & 13.4 & 34.7 & 21.8 & 22.0 & 1.8 & & 4.4 & 6.9 \\
\hline & & & 0.8 & 0.8 & 6.2 & 1.0 & 1.3 & 7.8 & 3.0 & 5.9 & 0.6 & & 0.4 & 0.5 \\
\hline 0.5 & 1 & 25 & 2.1 & 3.8 & 15.6 & 21.4 & 3.6 & 11.2 & 35.6 & 3.8 & 17.8 & 23.1 & 0.8 & 18.2 \\
\hline & & & 0.2 & 0.2 & 0.5 & 7.1 & 0.4 & 1.1 & 9.5 & 0.1 & 7.4 & 9.0 & 0.1 & 4.3 \\
\hline 0.5 & 5 & 5 & 30.4 & 13.6 & 7.0 & 2.9 & 22.2 & 10.2 & 4.4 & 26.3 & 21.0 & 0.6 & 20.7 & 0.7 \\
\hline & & & 1.7 & 1.9 & 1.7 & 0.2 & 1.0 & 2.2 & 0.2 & 7.3 & 6.0 & & 0.9 & 0.1 \\
\hline 0.5 & 5 & 25 & 6.1 & 10.6 & 8.3 & 6.6 & 3.6 & 18.6 & 8.8 & 9.8 & 26.8 & 19.4 & 2.4 & 2.3 \\
\hline & & & 0.6 & 0.4 & 4.2 & 2.1 & 0.3 & 3.7 & 2.7 & 2.2 & 8.7 & 7.3 & 0.2 & 0.1 \\
\hline 0.5 & 25 & 25 & 29.4 & 12.5 & 0.7 & 1.8 & 22.0 & 1.4 & 2.5 & 9.3 & 9.2 & 22.6 & 22.1 & 0.5 \\
\hline & & & 2.0 & 2.0 & & 0.6 & 0.9 & 0.1 & 1.0 & 0.2 & 2.6 & 8.4 & 1.1 & \\
\hline 1 & 1 & 1 & 51.0 & 31.6 & 28.7 & 32.2 & 24.2 & 10.3 & 11.5 & 1.6 & & 0.1 & 33.9 & 21.8 \\
\hline & & & 2.4 & 2.8 & 1.6 & 1.6 & 2.6 & 1.6 & 1.8 & & & & 1.5 & 1.0 \\
\hline 1 & 1 & 25 & 5.1 & 8.3 & 25.2 & 23.8 & 3.2 & 12.1 & 16.0 & 9.3 & 4.1 & 4.7 & 2.7 & 28.4 \\
\hline & & & 0.3 & 0.4 & 0.7 & 5.6 & 0.3 & 0.6 & 6.3 & 0.1 & 4.1 & 4.7 & 0.1 & 6.5 \\
\hline 5 & 5 & 5 & 48.1 & 31.1 & 27.7 & 30.2 & 23.1 & 10.4 & 11.9 & 1.6 & & 0.1 & 33.7 & 21.7 \\
\hline & & & 2.7 & 2.8 & 1.5 & 1.6 & 2.5 & 1.6 & 1.9 & 0.1 & & & 1.5 & 1.0 \\
\hline 25 & 25 & 25 & 46.0 & 29.6 & 25.3 & 29.6 & 23.7 & 10.6 & 12.4 & 1.7 & & 0.1 & 34.0 & 21.1 \\
\hline & & & 2.8 & 3.0 & 1.7 & 1.8 & 2.7 & 1.7 & 2.0 & & & & 1.4 & 1.0 \\
\hline
\end{tabular}

Table 4.3: Relative deviation of SEPT and $\ell$-SEPT from OPT in percentages, with 3 job classes. The upper value in a cell is the deviation for SEPT, the lower one is the deviation for $\ell$-SEPT. In case the deviation from either SEPT or $\ell$-SEPT from the optimal policy is less than $0.1 \%$, the value has been surpressed in the table. 


\section{Chapter 5}

\section{Scheduling with Incomplete Information: an Empirical Evaluation $^{1}$}

\subsection{Introduction}

In this chapter, we consider the stochastic single machine scheduling problem to minimize the total sum of expected completion times. Similar to the Bayesian scheduling approach of Chapter 4, we assume that the parameters of the processing time distributions are unknown to the scheduler. In contrast, we assume that the scheduler has no initial beliefs about the values of these parameters. The Shortest Expected Processing Time rule and other (Bayesian) scheduling rules based upon knowledge of the expected processing times no longer work under this setting. By processing jobs and observing their realized processing times, we learn about the correct values of the parameters. As in the Bayesian scheduling setting, this learning should be conducted carefully to avoid high costs in terms of the waiting times of the still to be processed jobs.

Problem definition. There are two classes of jobs, $J_{1}$ and $J_{2}$, that have to be processed by a single machine. The number of jobs in these classes are $n_{1} \geq 1$ and $n_{2} \geq 1$, respectively, and all jobs are available for processing from the beginning. We consider two job settings: with preemption, in which jobs may be interrupted from processing and resumed later on, and without preemption, in which a job once started needs to be processed until completion. The processing time of a job in class $J_{i}$ is a random variable $X_{i}$, which is independently and exponentially distributed with parameter $\lambda_{i}>0, i \in\{1,2\}$. The actual processing time of a job only becomes known upon completion of this job. The objective is to schedule the processing of all jobs so as to minimize the total completion time in expectation, $\sum_{j} \mathbb{E}\left[C_{j}\right]$.

Distinguishing from traditional stochastic scheduling, we assume that the value of $\lambda_{i}$ is unknown. Moreover, we assume that the scheduler has no prior beliefs about the value of $\lambda_{i}$, which is different from the Bayesian scheduling approach of the previous chapter. Overall, the scheduler thus lacks all knowledge about the jobs of classes $J_{1}$ and $J_{2}$, except for knowing that

\footnotetext{
${ }^{1}$ This chapter is based on Correa, Marbán, and Verschae (2012).
} 
each job is drawn from some specific exponential distribution and knowing the exact processing time of a job when it is completed. Hence, we speak in this chapter of non-clairvoyant stochastic scheduling. As we will see below, there is a rich literature on non-clairvoyant scheduling, which all started by the pioneering paper of by Motwani, Phillips, and Torng [81]. To our knowledge, this chapter is, however, the first one to address a stochastic version of non-clairvoyant scheduling problems.

In this chapter, we propose several approximative scheduling policies for the problem under consideration, and we investigate their theoretical performance. Moreover, we compare the performance of these approximative policies for the problem with preemption in an experimental setting with a range of other scheduling policies for which we have no performance guarantees. All policies together are referred to as learning policies, since each one of them attempts to learn about the values of the parameters using the realized processing times of the past. Exact definitions of scheduling policies, both approximative and optimal, and of (worst case) performance guarantees, can be found in Chapter 4. As already mentioned in the introductory chapter, Rothkopf [94] and Pinedo [87] show that the optimal scheduling policy in our setting is equivalent to the traditional scheduling policy called Shortest Expected Processing Time (SEPT).

In the remainder of this chapter, we assume that $X_{1} \sim \exp (1)$ and $X_{2} \sim \exp (1 / \alpha)$ with $\alpha>0$, unless explicitly stated otherwise. Moreover, we use the following additional notation. The random variable for the processing time of the $j$ th job of class $J_{i}$ is denoted by $X_{i}^{j}$. In addition, we write $x_{i}^{j}$ for the actual processing time of the $j$ th job of class $J_{i}$. Finally, we denote the expected sum of completion times of the optimal solution given $n_{1}$ and $n_{2}$ jobs by $\mathbb{E}\left[\Pi^{*}\left(n_{1}, n_{2}, \alpha\right)\right]$, where $\left(n_{1}, n_{2}, \alpha\right)$ is a state vector encompassing all relevant information of the state the system is in.

Previous work. Research in the field of stochastic scheduling has been active for more than four decades [88]. In this chapter, we contribute to the literature by introducing uncertainty on the parameters of the processing time distributions. In that way, policies, such as the Shortest Expected Processing Time (SEPT), are no longer applicable, which calls for the need to develop and study new ways of approaching these problems. In one line of research, a Bayesian framework is adopted in which the uncertainty is modeled by probability distributions on the unknown parameters. Although Bayesian techniques are widely applied in the area of inventory management and dynamic pricing (see Lariviere and Porteus [65] and Araman and Caldentey [6]), there are only few papers on this topic in the field of stochastic scheduling. For a detailed discussion of these papers, we refer the reader to the introduction of Chapter 4.

Another line of research approaches the uncertain processing times in a robust way, meaning that scheduling policies are developed for which the quality is stable over a wide range of possible scenarios. Consider, for example, the paper of Yang and Yu [115], in which a single machine scheduling problems is studied with as objective to minimize the sum of completion times. Following the results of Kouvelis and Yu [63] on general robust optimization problems, the processing time uncertainties are modeled by a scenario set, and several robustness measures are introduced. Subsequently, Yang and Yu show that the robust version of the problem under consideration is NP-complete, derive a dynamic programming formulation, and give 
two polynomial time heuristics. Another paper on the robust approach for the single machine scheduling problem is by Briand, La, and Erschler [20]. They develop robust optimal policies for the setting in which potential disruptions may occur during the scheduling execution.

A last line of research that deals with the uncertainty of processing times is referred to as non-clairvoyant scheduling. The first paper to study preemptive, non-clairvoyant scheduling on a single machine is by Motwani, Phillips, and Torng [81]. For the online problem with the objective to minimize the average waiting time of all $n$ jobs released at time zero, they show that the EQUI-partition policy [110], which partitions the machine evenly among the unfinished jobs and preempts only when jobs are completed, is $2-2 /(n+1)$-competitive. As mentioned by Edmonds [40], competitive ratios for non-clairvoyant scheduling problems are often unrealistically high. Therefore, it is custom in this area of research to give back an advantage to the non-clairvoyant scheduler. One way to do this could be to consider only jobs released at time zero, like in the papers of Edmonds, Chinn, Brecht, and Deng [41] and Motwani, Phillips, and Torng [81]. Another way is by allowing preemption, see, for example, the papers of Becchetti and Leonardi [11] and Deng, Gu, Brecht, and Lu [38]. The nonclairvoyant scheduler could also be equipped with a faster machine than the adversary such as in the papers of Bansal and Pruhs [9] and Kalyanasundaram and Pruhs [58]. Lastly, remark that the uncertainty of the optimal policy regarding the processing times, as assumed in this chapter, could also be seen as a way of giving advantage to the non-clairvoyant scheduler.

Our contribution. Our main contribution is the theoretical and empirical study of the performance of several scheduling policies for a stochastic single machine scheduling problem with incomplete information on the processing times of the jobs. We assume that there are two job classes and that the parameters of the processing time distributions of these classes are unknown. For the problem without preemption, we introduce in Section 5.2 deterministic and randomized learning policies, also referred to as Class Comparison policies. We show that each of these policies has a tight performance guarantee. In Section 5.3, we discuss the problem with a preemptive job setting. We conjecture that there is a constant lower bound on the performance guarantee of all possible scheduling policies. In addition, we provide tight performance guarantees for the EQUI-partition policy and a preemptive version of the Class Comparison policies. Thereafter, we introduce several other learning policies for which we do not have any performance guarantees, and we compare the computational performance of all proposed scheduling policies.

\subsection{Scheduling without preemption}

In this section, we give constant performance guarantees for several deterministic and randomized policies. Moreover, we construct for each of these policies an instance showing that the obtained guarantee is tight.

\subsubsection{Deterministic policies}

In this subsection, we consider policies that do not use any randomization. Our first learning policy, Class Comparison, is for the case with $n_{1}=n_{2}$, while our second learning policy, Equal- 
ized Class Comparison, is for the case with $n_{1} \neq n_{2}$. Let $\Pi^{C C}$ and $\Pi^{E C C}$ denote the random variables for the sum of completion times of these two deterministic policies.

Class Comparison (CC). Schedule one job of class $J_{1}$ and after that one job of class $J_{2}$. If $x_{1}^{1}<x_{2}^{1}$, first process all remaining jobs of class $J_{1}$ and then all remaining jobs of class $J_{2}$. Otherwise, process the job classes the other way around.

Theorem 5.2.1. Let $n_{1}=n_{2}$. Then, the Class Comparison policy is a 2-approximation for the stochastic single machine problem with two job classes and exponential processing time distributions with unknown parameters.

Proof. Let $n_{1}=n_{2}=n$. By (5.8) and (5.9) in Appendix 5.5.1, we get

$$
\begin{aligned}
\mathbb{E}\left[\Pi^{C C}(n, n, \alpha)\right]= & 2 n \mathbb{E}\left[X_{1}\right]+(2 n-1) \mathbb{E}\left[X_{2}\right]+\frac{(n-1) n}{2}\left(\mathbb{E}\left[X_{1}\right]+\mathbb{E}\left[X_{2}\right]\right) \\
& +\operatorname{Pr}\left[X_{1}^{1} \leq X_{2}^{1}\right](n-1)^{2} \mathbb{E}\left[X_{1}\right]+\operatorname{Pr}\left[X_{1}^{1}>X_{2}^{1}\right](n-1)^{2} \mathbb{E}\left[X_{2}\right] \\
= & n+(n-1) \alpha+\frac{n(n+1)}{2}(1+\alpha)+2(n-1)^{2} \frac{\alpha}{1+\alpha}
\end{aligned}
$$

Furthermore, we have

$$
\begin{aligned}
\mathbb{E}\left[\Pi^{*}(n, n, \alpha)\right] & =\frac{n(n+1)}{2}\left(\mathbb{E}\left[X_{1}\right]+\mathbb{E}\left[X_{2}\right]\right)+n^{2} \min \left\{\mathbb{E}\left[X_{1}\right], \mathbb{E}\left[X_{2}\right]\right\} \\
& =\frac{n(n+1)}{2}(1+\alpha)+n^{2} \min \{1, \alpha\},
\end{aligned}
$$

Combining these two expressions, we find

$$
\begin{aligned}
\frac{\mathbb{E}\left[\Pi^{C C}(n, n, \alpha)\right]}{\mathbb{E}\left[\Pi^{*}(n, n, \alpha)\right]} & \leq 1+\max _{\substack{\alpha>0 \\
n \geq 1}}\left\{\frac{n+(n-1) \alpha+2(n-1)^{2} \frac{\alpha}{1+\alpha}-n^{2} \min \{1, \alpha\}}{\frac{n(n+1)}{2}(1+\alpha)+n^{2} \min \{1, \alpha\}}\right\} \\
& \leq 1+\max _{\substack{\alpha>0 \\
n \geq 1}}\left\{\frac{n(1+\alpha)+n^{2} \frac{\alpha}{1+\alpha}}{\frac{n(n+1)}{2}(1+\alpha)+n^{2} \min \{1, \alpha\}}\right\} \\
& =1+\max _{\substack{\alpha>0 \\
n \geq 1}}\left\{\frac{(1+\alpha)+n \frac{\alpha}{1+\alpha}}{\frac{(n+1)}{2}(1+\alpha)+n \min \{1, \alpha\}}\right\} \\
& \leq 1+\max _{\alpha>0}\left\{\frac{(1+\alpha)+\frac{\alpha}{1+\alpha}}{(1+\alpha)+\min \{1, \alpha\}}\right\}<2,
\end{aligned}
$$

where the second and fourth inequality follow from $\frac{\alpha}{1+\alpha}<\min \{1, \alpha\}$ for every $\alpha \geq 0$, and the third one from the fact that the ratio is decreasing in $n$.

When the CC policy decides to start with a job of class $J_{1}$, take $n_{1}=n_{2}$ small and let $\alpha$ approach zero. In that way, the policy is forced to make a mistake which it cannot overcome anymore since there are no jobs left. This gives the following lower bound on the performance guarantee. 
Theorem 5.2.2. There exist parameter settings such that

$$
\frac{\mathbb{E}\left[\Pi^{C C}\left(n_{1}, n_{2}, \alpha\right)\right]}{\mathbb{E}\left[\Pi^{*}\left(n_{1}, n_{2}, \alpha\right)\right]}>2-\epsilon
$$

for any $\epsilon>0$.

Proof. Let $\alpha<1$ and set $n_{1}=n_{2}=1$. Then,

$$
\lim _{\alpha \downarrow 0} \frac{\mathbb{E}\left[\Pi^{C C}(1,1, \alpha)\right]}{\mathbb{E}\left[\Pi^{*}(1,1, \alpha)\right]}=\lim _{\alpha \downarrow 0} \frac{2+\alpha}{1+2 \alpha}=2 .
$$

In case $n_{1} \neq n_{2}$, it is assumed without loss of generality that $n_{1}>n_{2}$. This brings us to our second learning policy.

Equalized Class Comparison (ECC). Schedule $n_{1}-n_{2}$ jobs of class $J_{1}$ followed by one job of class $J_{2}$. If $x_{1}^{1} \leq x_{2}^{1}$, first process all remaining jobs of class $J_{1}$ and then all remaining jobs of class $J_{2}$. Otherwise, process the job classes the other way around.

Theorem 5.2.3. Let $n_{1}>n_{2}$. Then, the Equalized Class Comparison policy is a 2-approximation for the stochastic single machine problem with two job classes and exponential processing time distributions with unknown parameters.

Proof. Let $n_{1}=n+k$ with integer $k \geq 1$ and $n_{2}=n$. By (5.8) and (5.9) in Appendix 5.5.1, we obtain

$$
\begin{aligned}
\mathbb{E}\left[\Pi^{E C C}(n+k, n, \alpha)\right]= & \frac{k(k+1)}{2} \mathbb{E}\left[X_{1}\right]+2 n k \mathbb{E}\left[X_{1}\right]+2 n \mathbb{E}\left[X_{2}\right] \\
& +\operatorname{Pr}\left[X_{1}^{1} \leq X_{2}^{1}\right]\left(\frac{n(n+1)}{2} \mathbb{E}\left[X_{1}\right]+\frac{(n-1) n}{2} \mathbb{E}\left[X_{2}\right]+(n-1) n \mathbb{E}\left[X_{1}\right]\right) \\
& +\operatorname{Pr}\left[X_{1}^{1}>X_{2}^{1}\right]\left(\frac{n(n+1)}{2} \mathbb{E}\left[X_{1}\right]+\frac{(n-1) n}{2} \mathbb{E}\left[X_{2}\right]+(n-1) n \mathbb{E}\left[X_{2}\right]\right) \\
= & \frac{k(k+1)}{2}+2 n k+2 n \alpha+\frac{n(n+1)}{2}+\frac{(n-1) n}{2} \alpha+2(n-1) n \frac{\alpha}{1+\alpha} \\
= & \frac{k(k+1)}{2}+2 n k+n \alpha+\frac{n(n+1)}{2}(1+\alpha)+2(n-1) n \frac{\alpha}{1+\alpha}
\end{aligned}
$$

Further, we have

$$
\begin{aligned}
\mathbb{E}\left[\Pi^{*}(n+k, n, \alpha)\right] & =\frac{(n+k)(n+k+1)}{2} \mathbb{E}\left[X_{1}\right]+\frac{n(n+1)}{2} \mathbb{E}\left[X_{2}\right]+n(n+k) \min \left\{\mathbb{E}\left[X_{1}\right], \mathbb{E}\left[X_{2}\right]\right\} \\
& =\frac{k(k+1)}{2}+\frac{n(n+1)}{2}(1+\alpha)+n k+\left(n^{2}+n k\right) \min \{1, \alpha\} .
\end{aligned}
$$

Assume that $\mathbb{E}\left[X_{1}\right]>\mathbb{E}\left[X_{2}\right]$, such that it is optimal to process all jobs of class $J_{2}$ first. 
This gives

$$
\begin{aligned}
\frac{\mathbb{E}\left[\Pi^{E C C}(n+k, n, \alpha)\right]}{\mathbb{E}\left[\Pi^{*}(n+k, n, \alpha)\right]} & \leq 1+\max _{\substack{0<\alpha<1 ; \\
k \geq 1 ; n \geq 1}}\left\{\frac{n k+n \alpha+2(n-1) n \frac{\alpha}{1+\alpha}-n^{2} \alpha-n k \alpha}{\frac{k(k+1)}{2}+\frac{n(n+1)}{2}(1+\alpha)+n k+n^{2} \alpha+n k \alpha}\right\} \\
& =1+\max _{\substack{0<\alpha<1 ; \\
k \geq 1 ; n \geq 1}}\left\{\frac{n k+n^{2} \alpha+n \alpha^{2}-n \alpha-n^{2} \alpha^{2}-n k \alpha^{2}}{(1+\alpha)\left(\frac{k(k+1)}{2}+\frac{n(n+1)}{2}(1+\alpha)+n k+n^{2} \alpha+n k \alpha\right)}\right\} \\
& \leq 1+\max _{\substack{0<\alpha<1 ; \\
k \geq 1 ; n \geq 1}}\left\{\frac{n k+n^{2} \alpha+n \alpha^{2}}{n k+(1+\alpha) n^{2}}\right\}<2 .
\end{aligned}
$$

Next, assume that $\mathbb{E}\left[X_{1}\right] \leq \mathbb{E}\left[X_{2}\right]$. Then,

$$
\begin{aligned}
\frac{\mathbb{E}\left[\Pi^{E C C}(n+k, n, \alpha)\right]}{\mathbb{E}\left[\Pi^{*}(n+k, n, \alpha)\right]} & \leq 1+\max _{\substack{\alpha \geq 1 ; \\
k \geq 1 ; n \geq 1}}\left\{\frac{n \alpha+2(n-1) n \frac{\alpha}{1+\alpha}-n^{2}}{\frac{k(k+1)}{2}+\frac{n(n+1)}{2}(1+\alpha)+2 n k+n^{2}}\right\} \\
& =1+\max _{\substack{\alpha \geq 1 ; \\
k \geq 1 ; n \geq 1}}\left\{\frac{n \alpha^{2}+n^{2} \alpha-n \alpha-n^{2}}{(1+\alpha)\left(\frac{k(k+1)}{2}+\frac{n(n+1)}{2}(1+\alpha)+2 n k+n^{2}\right)}\right\} \\
& \leq 1+\max _{\substack{\alpha \geq 1 ; \\
n \geq 1}}\left\{\frac{n \alpha^{2}+n^{2} \alpha}{\frac{n(n+1)}{2} \alpha^{2}+n^{2} \alpha}\right\}<2,
\end{aligned}
$$

where the last inequality follows from the fact that the ratio is decreasing in $k$ and that $\frac{n^{2}+n}{2}>n$ for all $n \geq 1$.

Again we give a lower bound showing that this performance guarantee is tight. By assuming that $n_{1}=2$ and $n_{2}=1$, we force the ECC policy to alternate between classes, i.e., a job of class $J_{1}$, then a job of class $J_{2}$, and ending with a job of class $J_{1}$ (this is the only job left). The optimal policy, however, first processes all jobs of class $J_{1}$ followed by the single job of class $J_{2}$. The mistake of ECC is maximized by additionally assuming that $\mathbb{E}\left[X_{2}\right]=\alpha$ approaches infinity.

Theorem 5.2.4. There exist parameter settings such that

$$
\frac{\mathbb{E}\left[\Pi^{E C C}\left(n_{1}, n_{2}, \alpha\right)\right]}{\mathbb{E}\left[\Pi^{*}\left(n_{1}, n_{2}, \alpha\right)\right]}>2-\epsilon
$$

for any $\epsilon>0$.

Proof. Let $\alpha \geq 1$ and set $n_{1}=2, n_{2}=1$. Then,

$$
\lim _{\alpha \rightarrow \infty} \frac{\mathbb{E}\left[\Pi^{E C C}(2,1, \alpha)\right]}{\mathbb{E}\left[\Pi^{*}(2,1, \alpha)\right]}=\lim _{\alpha \rightarrow \infty} \frac{2 \alpha+4}{\alpha+5}=2
$$




\subsubsection{Randomized policies}

In this subsection, policies are allowed to randomize over the jobs scheduled at the machine. The first of these learning policies, Randomized Class Comparison, is for the case with $n_{1}=$ $n_{2}$, while the second learning policy, Randomized Class Comparison, is for the case with $n_{1} \neq$ $n_{2}$. Let $\Pi^{R C C}$ and $\Pi^{E R C C}$ denote the random variables for the sum of completion times of these two randomized policies.

Randomized Class Comparison (RCC). With probability $\frac{1}{2}$, schedule a job of class $J_{1}$ followed by a job of class $J_{2}$, and with probability $\frac{1}{2}$ schedule these two jobs vice versa. If $x_{1}^{1}<x_{2}^{1}$, first process all remaining jobs of class $J_{1}$ and then all remaining jobs of class $J_{2}$. Otherwise, process the job classes the other way around.

Theorem 5.2.5. Let $n_{1}=n_{2}$. Then, the Randomized Class policy is a $\frac{3}{2}$-approximation for the stochastic single machine problem with two job classes and exponential processing time distributions with unknown parameters.

Proof. Let $n_{1}=n_{2}=n+1$. By (5.8) and (5.9) in Appendix 5.5.1, we get

$$
\begin{aligned}
\mathbb{E}\left[\Pi^{R C C}(n+1, n+1, \alpha)\right]= & \left(2 n+\frac{3}{2}\right)\left(\mathbb{E}\left[X_{1}\right]+\mathbb{E}\left[X_{2}\right]\right) \\
& +\operatorname{Pr}\left[X_{1}^{1} \leq X_{2}^{1}\right]\left[\frac{n(n+1)}{2}\left(\mathbb{E}\left[X_{1}\right]+\mathbb{E}\left[X_{2}\right]\right)+n^{2} \mathbb{E}\left[X_{1}\right]\right] \\
& +\operatorname{Pr}\left[X_{1}^{1}>X_{2}^{1}\right]\left[\frac{n(n+1)}{2}\left(\mathbb{E}\left[X_{1}\right]+\mathbb{E}\left[X_{2}\right]\right)+n^{2} \mathbb{E}\left[X_{2}\right]\right] \\
= & (1+\alpha)\left(\frac{n(n+1)}{2}+2 n+\frac{3}{2}\right)+n^{2} \frac{2 \alpha}{1+\alpha} \\
= & (1+\alpha)\left(\frac{(n+1)(n+2)}{2}+n+\frac{1}{2}\right)+n^{2} \frac{2 \alpha}{1+\alpha},
\end{aligned}
$$

where the first equality follows from $\mathbb{E}\left[X_{i} \mid X_{i}^{1} \leq X_{j}^{1}\right]=\mathbb{E}\left[X_{i}\right]$ for $i \in\{1,2\}$. This holds since the jobs in both classes are independently distributed.

Assume without loss of generality that $\mathbb{E}\left[X_{1}\right]>\mathbb{E}\left[X_{2}\right]$, such that it is optimal to process all jobs of class $J_{2}$ first. Then,

$$
\begin{aligned}
\mathbb{E}\left[\Pi^{*}(n+1, n+1, \alpha)\right] & =\frac{(n+1)(n+2)}{2}\left(\mathbb{E}\left[X_{1}\right]+\mathbb{E}\left[X_{2}\right]\right)+(n+1)^{2} \mathbb{E}\left[X_{2}\right] \\
& =\frac{(n+1)(n+2)}{2}(1+\alpha)+(n+1)^{2} \alpha .
\end{aligned}
$$


This gives the following performance guarantee.

$$
\begin{aligned}
& \frac{\mathbb{E}\left[\Pi^{R C C}(n+1, n+1, \alpha)\right]}{\mathbb{E}\left[\Pi^{*}(n+1, n+1, \alpha)\right]} \leq \max _{\substack{0<\alpha<1 ; \\
n \geq 1}}\left\{\frac{(1+\alpha)[(n+1)(n+2)+2 n+1]+n^{2} \frac{4 \alpha}{1+\alpha}}{(n+1)(n+2)(1+\alpha)+2(n+1)^{2} \alpha}\right\} \\
& =1+\max _{\substack{0<\alpha<1 ; \\
n \geq 1}}\left\{\frac{-2 \alpha^{2} n^{2}-2 \alpha^{2} n+2 \alpha n^{2}-\alpha^{2}+2 n+1}{3 \alpha^{2} n^{2}+7 \alpha^{2} n+4 \alpha n^{2}+n^{2}+4 \alpha^{2}+10 \alpha n+3 n+6 \alpha+2}\right\} \\
& \leq 1+\max _{\substack{0<\alpha<1 ; \\
n \geq 1}}\left\{\frac{2 \alpha n^{2}+2 n+1}{4 \alpha n^{2}+n^{2}+3 n+2}\right\} \\
& \leq 1+\max _{\substack{0<\alpha<1 ; \\
n \geq 1}}\left\{\frac{2 \alpha n^{2}+2 n+1}{4 \alpha n^{2}+4 n+2}\right\} \leq \frac{3}{2},
\end{aligned}
$$

where the equality follows from multiplying both sides of the ratio by $(1+\alpha)$ and rewriting.

A lower bound, showing this performance guarantee is tight, can be constructed by setting both $n_{1}$ and $n_{2}$ equal to 2 and letting $\alpha$ approach zero. In that way, the policy half of time makes a mistake which it cannot overcome anymore.

Theorem 5.2.6. There exist parameter settings such that

$$
\frac{\mathbb{E}\left[\Pi^{R C C}\left(n_{1}, n_{2}, \alpha\right)\right]}{\mathbb{E}\left[\Pi^{*}\left(n_{1}, n_{2}, \alpha\right)\right]}>\frac{3}{2}-\epsilon
$$

for any $\epsilon>0$.

Proof. Let $\alpha<1$ and set $n_{1}=n_{2}=2$. Then,

$$
\lim _{\alpha \downarrow 0} \frac{\mathbb{E}\left[\Pi^{R C C}(2,2, \alpha)\right]}{\mathbb{E}\left[\Pi^{*}(2,2, \alpha)\right]}=\lim _{\alpha \downarrow 0} \frac{9+9 \alpha+\frac{4 \alpha}{1+\alpha}}{6+14 \alpha}=\frac{3}{2}
$$

Note that another way to end up at this lower bound is by taking $n_{1}=n_{2}=1$ such that in half of the time RCC schedules the jobs exactly like OPT, resulting in a ratio of 1 , and in half of the time it does not, giving a ratio of 2 .

For the case with $n_{1} \neq n_{2}$, it is again assumed that $n_{1}>n_{2}$. This brings us to our second randomized policy.

Equalized Randomized Class Comparison (ERCC). Schedule $n_{1}-n_{2}$ jobs of class $J_{1}$. Next, with probability $\frac{1}{2}$, schedule a job of class $J_{1}$ followed by a job of class $J_{2}$, and with probability $\frac{1}{2}$ schedule a single job of class $J_{2}$. If $x_{1}^{1} \leq x_{2}^{1}$, first process all remaining jobs of class $J_{1}$ and then all remaining jobs of class $J_{2}$. Otherwise, process the job classes the other way around.

Theorem 5.2.7. Let $n_{1} \neq n_{2}$. Then, the Equalized Randomized Class Comparison policy is a 2approximation for the stochastic single machine problem with two job classes and exponential processing time distributions with unknown parameters. 
Proof. Let $n_{1}=n+k$ with integer $k \geq 1$ and $n_{2}=n$. By (5.8) and (5.9) in Appendix 5.5.1, we find

$$
\begin{aligned}
\mathbb{E}\left[\Pi^{E R C C}(n+k, n, \alpha)\right]=\frac{k(k+1)}{2} \mathbb{E}\left[X_{1}\right]+2 n k \mathbb{E}\left[X_{1}\right]+\frac{1}{2}\left[2 n \mathbb{E}\left[X_{1}\right]+(2 n-1) \mathbb{E}\left[X_{2}\right]\right. \\
\quad+\operatorname{Pr}\left[X_{1}^{1} \leq X_{2}^{1}\right]\left(\frac{(n-1) n}{2}\left(\mathbb{E}\left[X_{1}\right]+\mathbb{E}\left[X_{2}\right]\right)+(n-1)^{2} \mathbb{E}\left[X_{1}\right]\right) \\
\left.\quad+\operatorname{Pr}\left[X_{1}^{1}>X_{2}^{1}\right]\left(\frac{(n-1) n}{2}\left(\mathbb{E}\left[X_{1}\right]+\mathbb{E}\left[X_{2}\right]\right)+(n-1)^{2} \mathbb{E}\left[X_{2}\right]\right)\right]+\frac{1}{2}\left[2 n \mathbb{E}\left[X_{2}\right]\right. \\
\quad+\operatorname{Pr}\left[X_{1}^{1} \leq X_{2}^{1}\right]\left(\frac{(n-1) n}{2} \mathbb{E}\left[X_{2}\right]+\frac{n(n+1)}{2} \mathbb{E}\left[X_{1}\right]+(n-1) n \mathbb{E}\left[X_{1}\right]\right) \\
\left.\quad+\operatorname{Pr}\left[X_{1}^{1}>X_{2}^{1}\right]\left(\frac{(n-1) n}{2} \mathbb{E}\left[X_{2}\right]+\frac{n(n+1)}{2} \mathbb{E}\left[X_{1}\right]+(n-1) n \mathbb{E}\left[X_{2}\right]\right)\right] \\
=\frac{k(k+1)}{2}+\frac{n(n+1)}{2}(1+\alpha)+2 n k+\frac{1}{2} n-\frac{1}{2} \alpha+n \alpha+(n-1)(2 n-1) \frac{\alpha}{1+\alpha} .
\end{aligned}
$$

Further, we have

$$
\begin{aligned}
\mathbb{E}\left[\Pi^{*}(n+k, n, \alpha)\right]= & \frac{(n+k)(n+k+1)}{2} \mathbb{E}\left[X_{1}\right]+\frac{n(n+1)}{2} \mathbb{E}\left[X_{2}\right] \\
& +n(n+k) \min \left\{\mathbb{E}\left[X_{1}\right], \mathbb{E}\left[X_{2}\right]\right\} \\
= & \frac{(n+k)(n+k+1)}{2}+\frac{n(n+1)}{2} \alpha+n(n+k) \min \{1, \alpha\} \\
= & \frac{k(k+1)}{2}+\frac{n(n+1)}{2}(1+\alpha)+n k+\left(n^{2}+n k\right) \min \{1, \alpha\} .
\end{aligned}
$$

Assume that $\mathbb{E}\left[X_{1}\right]>\mathbb{E}\left[X_{2}\right]$, such that it is optimal to process all jobs of class $J_{2}$ first. Then,

$$
\begin{aligned}
\frac{\mathbb{E}\left[\Pi^{E R C C}(n+k, n, \alpha)\right]}{\mathbb{E}\left[\Pi^{*}(n+k, n, \alpha)\right]} & \leq 1+\max _{\substack{0<\alpha<1 ; \\
k \geq 1 ; n \geq 1}}\left\{\frac{n k+\frac{1}{2} n-\frac{1}{2} \alpha+n \alpha+(n-1)(2 n-1) \frac{\alpha}{1+\alpha}-n^{2} \alpha-n k \alpha}{\frac{k(k+1)}{2}+\frac{n(n+1)}{2}(1+\alpha)+n k+n^{2} \alpha+n k \alpha}\right\} \\
& \leq 1+\max _{\substack{0<\alpha<1 ; \\
k \geq 1 ; n \geq 1}}\left\{\frac{n k+\frac{1}{2} n+\frac{1}{2} \alpha+n \alpha^{2}-n^{2} \alpha^{2}+n^{2} \alpha}{(1+\alpha)\left(\frac{k(k+1)}{2}+\frac{n(n+1)}{2}(1+\alpha)+n k+n^{2} \alpha+n k \alpha\right)}\right\} \\
& \leq 1+\max _{\substack{0<\alpha<1 ; \\
k \geq 1 ; n \geq 1}}\left\{\frac{\left(n k+\frac{1}{2} n\right)+\frac{1}{2} \alpha+n^{2} \alpha}{\left(\frac{k(k+1)}{2}+\frac{n(n+1)}{2}+n k\right)+n \alpha+\left(n^{2} \alpha+n^{2} \alpha\right)}\right\} \\
& \leq 1+\max _{\substack{0<\alpha<1 ; \\
k \geq 1 ; n \geq 1}}\left\{\frac{\left(n k+\frac{1}{2} n\right)+\frac{1}{2} \alpha+n^{2} \alpha}{2\left(n k+\frac{1}{2} n\right)+\alpha+2 n^{2} \alpha}\right\}=\frac{3}{2}<2,
\end{aligned}
$$

where the second inequality follows from multiplying both sides of the ratio by $(1+\alpha)$ and leaving out the negative terms in the numerator, and the fourth one from the reasoning below.

$$
2\left(n k+\frac{1}{2} n\right) \leq \frac{1}{2} k^{2}+\frac{1}{2} n^{2}+n k+\frac{1}{2} n+\frac{1}{2} k \Leftrightarrow 0 \leq \frac{1}{2}\left[(k-n)^{2}+(k-n)\right],
$$

which holds for any integer $k \geq 1$ and integer $n \geq 1$. 
Next, assume that $\mathbb{E}\left[X_{1}\right] \leq \mathbb{E}\left[X_{2}\right]$. This gives

$$
\begin{aligned}
\frac{\mathbb{E}\left[\Pi^{E R C C}(n+k, n, \alpha)\right]}{\mathbb{E}\left[\Pi^{*}(n+k, n, \alpha)\right]} & \leq 1+\max _{\substack{\alpha \geq 1 ; \\
k \geq 1 ; n \geq 1}}\left\{\frac{\frac{1}{2} n-\frac{1}{2} \alpha+n \alpha+(n-1)(2 n-1) \frac{\alpha}{1+\alpha}-n^{2}}{\frac{k(k+1)}{2}+\frac{n(n+1)}{2}(1+\alpha)+2 n k+n^{2}}\right\} \\
& =1+\max _{\substack{\alpha \geq i \\
n \geq 1}}\left\{\frac{n^{2} \alpha+n \alpha^{2}+\frac{1}{2} \alpha-\frac{1}{2} \alpha^{2}+\frac{1}{2} n-n^{2}-\frac{3}{2} n \alpha}{(1+\alpha)\left(1+\frac{n(n+1)}{2}(1+\alpha)+2 n+n^{2}\right)}\right\} \\
& \leq 1+\max _{\substack{\alpha \geq 1 \\
n \geq 1}}\left\{\frac{n^{2} \alpha+n \alpha^{2}+\frac{1}{2} \alpha}{n^{2} \alpha+\frac{n(n+1)}{2} \alpha^{2}+\frac{1}{2} \alpha}\right\}<2,
\end{aligned}
$$

where the equality follows from multiplying both sides of the ratio by $(1+\alpha)$ and observing that it is decreasing in $k$, and the last inequality from $\frac{n(n+1)}{2} \geq n$ for all $n \geq 1$.

Note that we disregard many terms in the proof of Theorem 5.2.7 when $\mathbb{E}\left[X_{1}\right] \leq \mathbb{E}\left[X_{2}\right]$, which could possibly improve the bound on the performance guarantee. Therefore, we conjecture that the actual performance guarantee of ERCC is smaller.

Conjecture 5.2.8. Let $n_{1} \neq n_{2}$. Then, the Equalized Randomized Class Comparison policy is a $\frac{3}{2}$-approximation for the stochastic single machine problem with two job classes and exponential processing time distributions with unknown parameters.

We end the section by giving a lower bound of $3 / 2$ on the performance guarantee of the ERCC policy. Like in the deterministic setting, we assume that $n_{1}=2$ and $n_{2}=1$ such that there is a $50 \%$ chance that the policy alternates between classes instead of first processing all jobs of class $J_{1}$ followed by the single job of class $J_{2}$, which would be optimal. This mistake is maximized when $\mathbb{E}\left[X_{2}\right]=\alpha$ goes to infinity.

Theorem 5.2.9. There exist parameter settings such that

$$
\frac{\mathbb{E}\left[\Pi^{E R C C}\left(n_{1}, n_{2}, \alpha\right)\right]}{\mathbb{E}\left[\Pi^{*}\left(n_{1}, n_{2}, \alpha\right)\right]}>\frac{3}{2}-\epsilon
$$

for any $\epsilon>0$.

Proof. Let $\alpha \geq 1$ and set $n_{1}=2, n_{2}=1$. Then,

$$
\lim _{\alpha \rightarrow \infty} \frac{\mathbb{E}\left[\Pi^{E R C C}(2,1, \alpha)\right]}{\mathbb{E}\left[\Pi^{*}(2,1, \alpha)\right]}=\lim _{\alpha \rightarrow \infty} \frac{1+(1+\alpha)+2+\frac{1}{2}-\frac{1}{2} \alpha+\alpha}{1+(1+\alpha)+2+1}=\lim _{\alpha \rightarrow \infty} \frac{\frac{3}{2} \alpha+\frac{9}{2}}{\alpha+5}=\frac{3}{2} .
$$

\subsection{Scheduling with preemption}

In this section, we analyze the theoretical and empirical performance of policies that have the ability to schedule jobs in parallel by using time-multiplexing, i.e., by continuously executing arbitrary small slices of the jobs. As we will see, this enables us to use much more sophisticated learning strategies over the jobs in the two classes. 


\subsubsection{Learning policies with performance guarantees}

In this subsection, we give constant tight performance guarantees for policies in a setting in which preemption of jobs is allowed. First, we show that EQUI-partition and a preemptive version of the Class Comparison policy always have the same expected sum of completion times. Let $\Pi^{E Q U I}$ and $\Pi^{P C C}$ denote the random variables for the sum of completion times of these two policies.

EQUI-partition (EQUI). Partition the machine evenly among all jobs. As soon as a job is finished, re-partition the machine evenly among all jobs that are not finished yet. Continue in this fashion until all jobs are processed.

Preemptive Class Comparison (PCC). Schedule a job of class $J_{1}$ and a job of class $J_{2}$ in parallel, and preempt as soon as one of both jobs finishes. Then, schedule all remaining jobs of the class that finished first followed by the jobs of the other class.

Lemma 5.3.1. For any $n_{1}, n_{2} \geq 1$ and $\alpha>0$,

$$
\mathbb{E}\left[\Pi^{E Q U I}\left(n_{1}, n_{2}, \alpha\right)\right]=\mathbb{E}\left[\Pi^{P C C}\left(n_{1}, n_{2}, \alpha\right)\right] .
$$

Proof. Let $J_{1}=\left\{1, \ldots, n_{1}\right\}$ and $J_{2}=\left\{n_{1}+1, \ldots, n_{1}+n_{2}\right\}$. Further, let $X^{j}$ denote a random variable for the processing time of job $j$, defined as follows:

$$
X^{j}=\left\{\begin{array}{lll}
X_{1}^{j} & \text { if } & j \in J_{1}, \\
X_{2}^{j} & \text { if } & j \in J_{2} .
\end{array}\right.
$$

The completion time of job $l$ when using EQUI is

$$
\sum_{m \in J_{1} \cup J_{2}} \mathbb{E}\left[\min \left\{X^{l}, X^{m}\right\}\right]
$$

since it depends on all completion times of the jobs that finish before this job. For the EQUIpartition policy, we then get

$$
\begin{aligned}
\mathbb{E}\left[\Pi^{E Q U I}\left(n_{1}, n_{2}, \alpha\right)\right]= & \sum_{l \in J_{1} \cup J_{2}} \sum_{m \in J_{1} \cup J_{2}} \mathbb{E}\left[\min \left\{X^{l}, X^{m}\right\}\right] \\
= & \sum_{l \in J_{1}}\left[\sum_{m \in J_{1}} \mathbb{E}\left[\min \left\{X^{l}, X^{m}\right\}\right]+\sum_{m \in J_{2}} \mathbb{E}\left[\min \left\{X^{l}, X^{m}\right\}\right]\right] \\
& +\sum_{l \in J_{2}}\left[\sum_{m \in J_{1}} \mathbb{E}\left[\min \left\{X^{l}, X^{m}\right\}\right]+\sum_{m \in J_{2}} \mathbb{E}\left[\min \left\{X^{l}, X^{m}\right\}\right]\right] \\
= & \sum_{l \in J_{1}} \sum_{m \in J_{1} \backslash\{l\}} \frac{1}{2} \mathbb{E}\left[X_{1}\right]+\sum_{l \in J_{1}} \mathbb{E}\left[X_{1}\right]+\sum_{l \in J_{1}} \sum_{m \in J_{2}} \frac{\mathbb{E}\left[X_{1}\right] \mathbb{E}\left[X_{1}\right]+\mathbb{E}\left[X_{2}\right]}{\mathbb{E}\left[X_{1}\right] \mathbb{E}\left[X_{2}\right]} \\
& +\sum_{l \in J_{2}} \sum_{m \in J_{1}} \frac{\mathbb{E}\left[X_{1}\right]+\mathbb{E}\left[X_{2}\right]}{\mathbb{E}} \sum_{l \in J_{2}} \frac{1}{2} \mathbb{E}\left[X_{2}\right]+\sum_{l \in J_{2} \backslash\{l\}} \mathbb{E}\left[X_{2}\right]
\end{aligned}
$$




$$
\begin{aligned}
& =\frac{n_{1}\left(n_{1}-1\right)}{2}+n_{1}+n_{1} n_{2} \frac{\alpha}{1+\alpha}+\frac{n_{2}\left(n_{2}-1\right)}{2} \alpha+n_{2} \alpha+n_{1} n_{2} \frac{\alpha}{1+\alpha} \\
& =\frac{n_{1}\left(n_{1}+1\right)}{2}+\frac{n_{2}\left(n_{2}+1\right)}{2} \alpha+2 n_{1} n_{2} \frac{\alpha}{1+\alpha} .
\end{aligned}
$$

where the third equality follows from (5.10) and (5.10) in Appendix 5.5.1.

For the PCC policy, we obtain

$$
\begin{aligned}
\mathbb{E} & {\left[\Pi^{P C C}\left(n_{1}, n_{2}, \alpha\right)\right]=2\left(n_{1}+n_{2}\right) \mathbb{E}\left[\min \left\{X_{1}^{1}, X_{2}^{1}\right\}\right] } \\
& +\operatorname{Pr}\left[X_{1}^{1} \leq X_{2}^{1}\right]\left(\frac{\left(n_{1}-1\right) n_{1}}{2} \mathbb{E}\left[X_{1}\right]+\frac{n_{2}\left(n_{2}+1\right)}{2} \mathbb{E}\left[X_{2}\right]+\left(n_{1}-1\right) n_{2} \mathbb{E}\left[X_{1}\right]\right) \\
& +\operatorname{Pr}\left[X_{1}^{1}>X_{2}^{1}\right]\left(\frac{n_{1}\left(n_{1}+1\right)}{2} \mathbb{E}\left[X_{1}\right]+\frac{\left(n_{2}-1\right) n_{2}}{2} \mathbb{E}\left[X_{2}\right]+n_{1}\left(n_{2}-1\right) \mathbb{E}\left[X_{2}\right]\right) \\
= & 2\left(n_{1}+n_{2}\right) \frac{\alpha}{1+\alpha}+\frac{\alpha}{1+\alpha}\left(\frac{\left(n_{1}-1\right) n_{1}}{2}+\frac{n_{2}\left(n_{2}+1\right)}{2} \alpha+\left(n_{1}-1\right) n_{2}\right) \\
& +\frac{1}{1+\alpha}\left(\frac{n_{1}\left(n_{1}+1\right)}{2}+\frac{\left(n_{2}-1\right) n_{2}}{2} \alpha+n_{1}\left(n_{2}-1\right) \alpha\right) \\
= & \frac{n_{1}\left(n_{1}+1\right)}{2}+\frac{n_{2}\left(n_{2}+1\right)}{2} \alpha+2 n_{1} n_{2} \frac{\alpha}{1+\alpha},
\end{aligned}
$$

where the first equality follows from (5.12), and the second one from (5.8) and (5.9) in Appendix 5.5.1. Observing that (5.1) and (5.2) are identical concludes the proof.

The following theorem presents constant performance guarantees for the EQUI-partition and PCC policy.

Theorem 5.3.2. The EQUI and PCC policy are both a 1.20711-approximation for the stochastic single machine problem with two job classes and exponential processing time distributions with unknown parameters. If also $n_{1}=n_{2}$, then the performance guarantee decreases to 1.17157 .

Proof. Define $\beta=n_{1} / n_{2}$ and assume without loss of generality that $\mathbb{E}\left[X_{1}\right]>\mathbb{E}\left[X_{2}\right]$, such that it is optimal to process all jobs of class $J_{2}$ first. From the proof of Lemma 5.3.1, it follows that the performance guarantee of both policies is bounded by

$$
\begin{aligned}
\max _{\substack{0<\alpha<1 ; \\
n_{1} \geq 1 ; n_{2} \geq 1}}\left\{\frac{\frac{n_{1}\left(n_{1}+1\right)}{2}+\frac{n_{2}\left(n_{2}+1\right)}{2} \alpha+2 n_{1} n_{2} \frac{\alpha}{1+\alpha}}{\frac{n_{1}\left(n_{1}+1\right)}{2}+\frac{n_{2}\left(n_{2}+1\right)}{2} \alpha+n_{1} n_{2} \alpha}\right\}=1+\max _{\substack{0<\alpha<1 ; \\
n_{1} \geq 1 ; n_{2} \geq 1}}\left\{\frac{2 n_{1} n_{2} \frac{\alpha}{1+\alpha}-n_{1} n_{2} \alpha}{\frac{n_{1}\left(n_{1}+1\right)}{2}+\frac{n_{2}\left(n_{2}+1\right)}{2} \alpha+n_{1} n_{2} \alpha}\right\} \\
=1+\max _{\substack{0<\alpha<1 ; \\
n_{1} \geq 1 ; n_{2} \geq 1}}\left\{\frac{2 \frac{\alpha}{1+\alpha}-\alpha}{\frac{n_{1}+1}{2 n_{2}}+\frac{n_{2}+1}{2 n_{1}} \alpha+\alpha}\right\} \leq 1+\max _{\substack{0<\alpha<1 ; \\
n_{1} \geq 1 ; n_{2} \geq 1}}\left\{\frac{2 \frac{\alpha}{1+\alpha}-\alpha}{\frac{n_{1}}{2 n_{2}}+\frac{n_{2}}{2 n_{1}} \alpha+\alpha}\right\} \\
=1+\max _{\substack{0<\alpha<1 ; \\
\beta \geq 0}}\left\{\frac{2 \alpha \beta(1-\alpha)}{(\alpha+1)\left(\beta^{2}+2 \alpha \beta+\alpha\right)}\right\} .
\end{aligned}
$$

In case $n_{1}=n_{2}$, and thus $\beta=1$, maximizing the ratio in the last expression over $0<\alpha<1$ shows that the worst case performance guarantee equals $4-2 \sqrt{2} \approx 1.17157$ and is attained when $\alpha=(2 \sqrt{2}-1) / 7 \approx 0.2612$. On the other hand, we find a performance guarantee of approximately 1.20711 when $n_{1} \neq n_{2}$, which is attained at $\alpha=3-2 \sqrt{2} \approx 0.1715$ and $\beta=$ $\sqrt{2}-1 \approx 0.4142$. 
From the first term in (5.3), it trivially follows that the performance guarantees of Theorem 5.3.2 are tight: choose the number of jobs such that $n_{1}=\beta n_{2}$. Next, set $\alpha=(2 \sqrt{2}-1) / 7$ and $\beta=1$ or set $\alpha=3-2 \sqrt{2}$ and $\beta=\sqrt{2}-1$, depending on whether the number of jobs in both classes is equal or not. Lastly, let $n_{2}$ approach infinity. This leads to the result below.

Theorem 5.3.3. For any $\epsilon_{1}, \epsilon_{2}>0$, there exist parameter settings such that

$$
\frac{\mathbb{E}\left[\Pi^{E Q U I}\left(n_{1}, n_{2}, \alpha\right)\right]}{\mathbb{E}\left[\Pi^{*}\left(n_{1}, n_{2}, \alpha\right)\right]}=\frac{\mathbb{E}\left[\Pi^{P C C}\left(n_{1}, n_{2}, \alpha\right)\right]}{\mathbb{E}\left[\Pi^{*}\left(n_{1}, n_{2}, \alpha\right)\right]}>1.17157-\epsilon_{1}
$$

in case $n_{1}=n_{2}$, and

$$
\frac{\mathbb{E}\left[\Pi^{E Q U I}\left(n_{1}, n_{2}, \alpha\right)\right]}{\mathbb{E}\left[\Pi^{*}\left(n_{1}, n_{2}, \alpha\right)\right]}=\frac{\mathbb{E}\left[\Pi^{P C C}\left(n_{1}, n_{2}, \alpha\right)\right]}{\mathbb{E}\left[\Pi^{*}\left(n_{1}, n_{2}, \alpha\right)\right]}>1.20711-\epsilon_{2}
$$

in case $n_{1} \neq n_{2}$.

\subsubsection{Learning policies without performance guarantees}

In this subsection, we introduce five learning policies for which we have no performance guarantees. The first two policies are an extension of PCC, while the last three are related to EQUI. For clarification, we also give a stochastic scheduling example in which we calculate the performance of all these policies.

The first learning policy is a direct extension of PCC, in which we no longer learn with one job but with $k$ jobs. Let $\Pi^{k-P C C}$ denote the random variable for the sum of completion times of this policy.

Preemptive Class Comparison with $k$ jobs ( $k$-PCC). Let a pair refer to a single job of class $J_{1}$ and $J_{2}$, processed in parallel. Schedule $k$ of these pairs sequentially, and preempt a pair every time one of its jobs finishes. Let $k_{1}$ and $k_{2}$ denote the number of jobs finished of class $J_{1}$ and $J_{2}$, respectively. Then, process the remaining jobs: (A) If $k_{1}>k_{2}$, schedule all jobs of class $J_{1}$ followed by the jobs of class $J_{2}$; (B) If $k_{1}<k_{2}$, schedule all jobs of class $J_{2}$ followed by the jobs of class $J_{1}$; (C) If $k_{1}=k_{2}$, schedule all jobs of class $J_{1}$ followed by the jobs of class $J_{2}$ with probability $1 / 2$, and process these job classes the other way around with probability $1 / 2$.

By learning with more than one job, we receive additional information on the processing times and as such decrease the probability that we make a "wrong" decision. On the other hand, learning with too many jobs may result in a high objective value, since we postpone decision making. This trade-off will be discussed in more depth in Section 5.3.3. We, however, conjecture that it is always better to learn with an odd number of jobs.

Conjecture 5.3.4. Let $k_{1} \in \mathbb{N}_{+}$be an even number. Then, there always exists an odd number $k_{2} \in \mathbb{N}_{+}$such that

$$
\mathbb{E}\left[\Pi^{k_{1}-P C C}\left(n_{1}, n_{2}, \alpha\right)\right] \geq \mathbb{E}\left[\Pi^{k_{2}-P C C}\left(n_{1}, n_{2}, \alpha\right)\right],
$$

for any $n_{1}, n_{2} \geq 1$ and $\alpha>0$. 
In case we no longer compare the individual processing times, but the average processing times of each class over $k$ jobs, we end up at our second learning policy. This policy is based on the fact that the average processing time is an unbiased estimator of the parameter of the processing time distribution.

Average Preemptive Class Comparison with $k$ jobs ( $k$-APCC). Assign $1 / 2$ of the machine to class $J_{1}$ and $1 / 2$ to class $J_{2}$. Then, schedule on each class partition $k$ jobs sequentially and preempt as soon as all $k$ jobs of one class are finished. Let $f_{1}$ and $f_{2}$ denote the number of jobs finished of class $J_{1}$ and $J_{2}$, respectively. Further, let $x_{i}^{j}=0$ when job $j$ of class $J_{i}$ is not finished. If $f_{2}=0$ or

$$
\frac{\sum_{j=1}^{k} x_{1}^{j}}{f_{1}} \leq \frac{\sum_{j=1}^{k} x_{2}^{j}}{f_{2}}
$$

schedule all remaining jobs of class $J_{1}$ followed by the jobs of class $J_{2}$. Otherwise, schedule the job classes the other way around.

A possible weak point of this policy is the large variance of the sample mean for small $k$, which makes decision errors very likely. We note, however, that this policy is identical to PCC for $k=1$ and that it uses more information when $k>1$. Therefore, we expect these decision errors to be reasonably bounded. We will consider this issue more carefully in Section 5.3.3.

The third learning policy is a mutation of EQUI. In order to give more power to the class that finishes its jobs the fastest, we keep the proportion of the machine assigned to each class constant.

Constant EQUI-partition (CEQUI). Assign $n_{1} /\left(n_{1}+n_{2}\right)$ of the machine to class $J_{1}$ and $n_{2} /\left(n_{1}+n_{2}\right)$ to class $J_{2}$. Partition the assigned fraction evenly among all jobs of the corresponding class. As soon as a job of a certain class is finished, re-partition the remaining jobs of that class evenly among the corresponding class partition. Preempt as soon as the jobs of one class are finished, and schedule the remaining jobs sequentially on the full machine.

Using CEQUI, but forcing the machine into a decision after processing $k$ jobs of some class gives us our fourth learning policy.

Constant EQUI-partition with $k$ jobs ( $k$-CEQUI) Schedule the jobs according to CEQUI, and preempt at time $t$ when $\max \left\{d_{1, t}, d_{2, t}\right\}>k$, where $d_{1, t}$ and $d_{2, t}$ denote the number of jobs finished up to time $t$ of class $J_{1}$ and $J_{2}$, respectively. If $d_{1, t}>k$, schedule all remaining jobs of class $J_{1}$ followed by the jobs of class $J_{2}$. Otherwise, schedule the job classes the other way around.

The fifth learning policy is based on the Proportional Sharing algorithm studied in the paper of Cole, Correa, Gkatzelis, Mirrokni, and Olver [28]. Each job receives a certain proportion of the machine, which is adjusted over time based on the knowledge obtained when jobs finish. Obviously, when more jobs of class $J_{1}$ are finished compared to class $J_{2}$, a larger proportion is given to the jobs of class $J_{1}$, and vice versa when it is the other way around. Note that EQUI is a special variant of this Proportional Learning (PLEARN) policy. 
Proportional Learning (PLEARN). At any time, schedule all unfinished jobs in parallel, and assign hereby to each job of class $J_{i}$ the following fraction of the machine

$$
\beta_{i}\left(c_{i}\right) /\left(\beta_{1}\left(c_{1}\right) \cdot\left(n_{1}-c_{1}\right)+\beta_{2}\left(c_{2}\right) \cdot\left(n_{2}-c_{2}\right)\right),
$$

where $c_{i}$ denotes the number of jobs finished of class $J_{i}$ so far, and $\beta_{1}\left(c_{1}\right)$ and $\beta_{2}\left(c_{2}\right)$ are functions depending on the number of jobs finished in each class.

For clarification, we discuss a scheduling example for each of these learning policies.

Example 1. Consider a stochastic scheduling problem with two job classes, $J_{1}$ and $J_{2}$. Let $n_{1}=7$ and $n_{2}=5$, and set $\lambda=1$ and $\lambda_{2}=4$. Figure 5.1 gives a graphical representation of the performance of all these policies. A solid line refers to a job that has finished processing, while a dashed line refers to a job that is preempted and not finished yet. The numbers in the gray and white area correspond to the processing times of the jobs of class $J_{1}$ and $J_{2}$, respectively. In case one of these numbers has a superscript, say $j$, it indicates the remaining processing time of job $j$ after it has been preempted. Moreover, the numbers below the machine stand for the completion times of the different jobs.

The Preemptive Class Comparison policy with $k=3$ jobs is given in Figure 5.1(a). As can be seen, the first 3 jobs of class $J_{2}$ finish before those of class $J_{1}$. Therefore, the policy continues by processing the last 2 jobs of class $J_{2}$, followed by what is left from the first 3 jobs of class $J_{1}$, and ending with the remaining jobs of class $J_{1}$. Summing up all the completion times of the jobs, we find a performance for 3-PCC that is equal to 27.1071. A slightly smaller objective value is obtained by the Average Preemptive Class Comparison policy with $k=2$ jobs. This is shown in Figure 5.1(b), where the first 2 jobs of class $J_{2}$ are done before the first job of class $J_{1}$ such that $f_{1}=0$. Hence, the policy schedules all remaining jobs of class $J_{1}$ before the jobs of class $J_{2}$. This gives a total sum of completion times of 27.0517 for 2-APCC.

Figure 5.1(c) presents the Constant EQUI-partition policy. It shows that class $J_{2}$ finishes processing at a completion time of 1.5362. At that moment, there are still five jobs of class $J_{1}$ left to be processed. The completion time 1.5362 is obtained by adding 0.3110 (what is left of the last job of class $J_{2}$ ) times $12 / 5$ (to scale back the processing time of this job) to 0.7898 (the time at which the last job of class $J_{2}$ starts processing). Further, the remaining time of the first of the unfinished jobs of class $J_{1}, 0.0636$, is calculated as follows: initially subtract $x_{1}^{2}=0.0989$ and $x_{1}^{4}=0.0906$ from $x_{1}^{1}=0.2049$, since the corresponding jobs of class $J_{1}$ are done before time 1.5362. Subsequently, subtract what is processed of the first job of $J_{1}$ after time 1.1726, which is (1.5362 - 1.1726) times $7 /(5 \cdot 12)$ (to scale back time). Overall, the total sum of completion times for CEQUI is 27.0459. A better performance of 25.9817 is obtained by 3-CEQUI, which can be seen in Figure 5.1(d). Reason for this is that the policy is forced to choose a class after a learning phase of 3 jobs, and it picks the correct one.

The Proportional Learning policy is given in Figure 5.1(e). Hereby, the following update scheme is used: every time a job of class $J_{i}$ finishes, $\beta_{i}$ is increased by 1.2. In that way, the policy initially gives a lot of power to class $J_{2}$, which has a few jobs with very small processing time. As soon as some jobs of class $J_{1}$ finish as well, this class slowly regains some of its lost machine fraction until class $J_{2}$ finishes completely at time 1.3031. This results in a total sum of completion times for PLEARN of 26.5702. The completion time of 0.1001 is found by adding 0.0014 (what is left of the second job of class $J_{2}$ that has just finished) times 


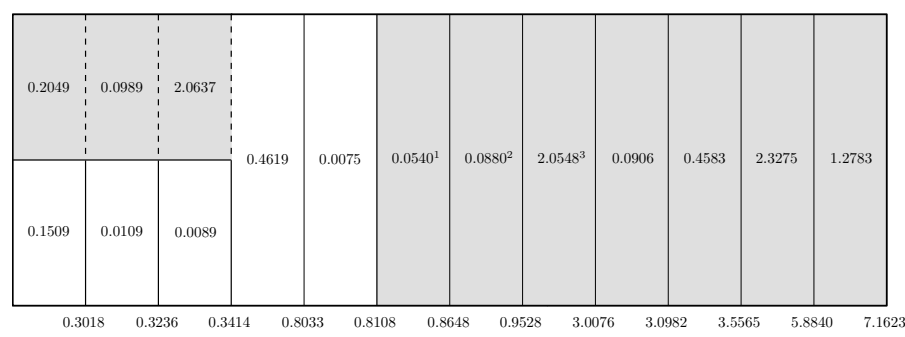

(a) Preemptive Class Comparison with 3 jobs

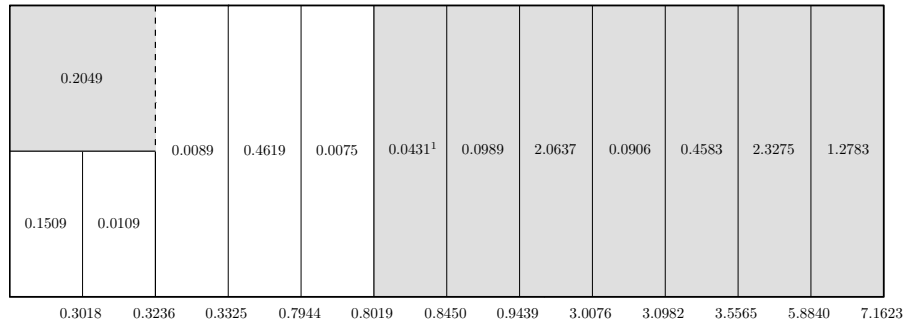

(b) Average Preemptive Class Comparison with 3 jobs

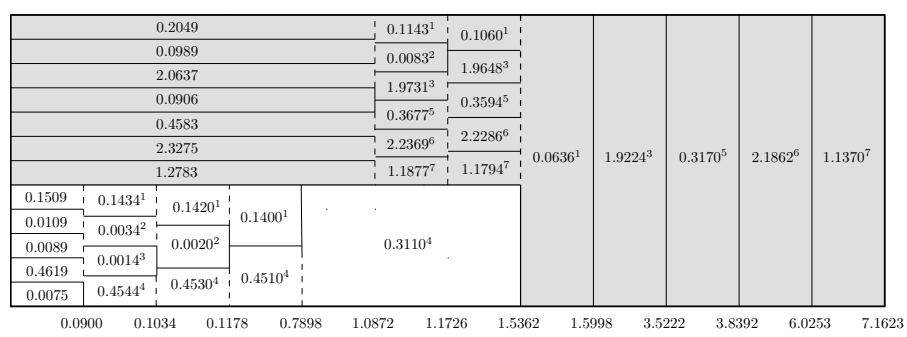

(c) Constant EQUI-partition

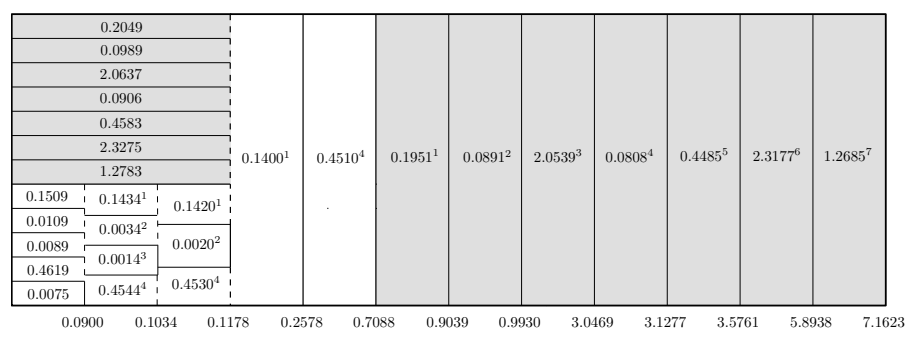

(d) Constant EQUI-partition with 3 jobs

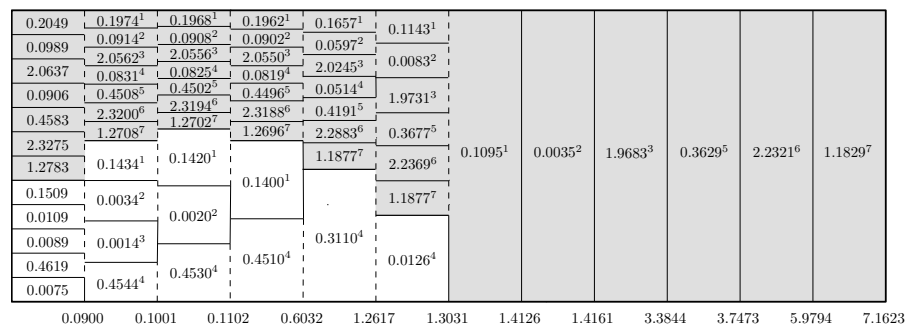

(e) Proportional Learning

Figure 5.1: Performance of the five learning policies in Example 1 
$\left(7 \beta_{1}+4 \beta_{2}\right) / \beta_{2}=15.8 / 2.2 \approx 0.0100$ to 0.0900 (the previous completion time). Further, the remaining processing time of the first job of class $J_{1}$ at time 0.1001 , i.e., 0.1968 , is obtained by subtracting 0.0014 times $\beta_{1} / \beta_{2}=1 / 2.2 \approx 0.4545$ (to scale the processing time back to class $J_{1}$ jobs) from 0.1974 (the remaining processing time of the job at the previous preemption). Note that scaling is not needed to get the remaining processing times of the jobs of class $J_{2}$ at time 0.1001 .

The optimal policy first processes the jobs of class $J_{2}$ followed by the jobs of class $J_{1}$, which leads to a total sum of completion times of 26.2536. Hence, the ratios of 3-PCC, 2-APCC, CEQUI, 3-CEQUI, and PLEARN to the optimal policy are, respectively, 1.0325, 1.0304, 1.0302, 0.9900 , and 1.0121. Note that the fourth performance guarantee is below 1 . This is explained by the fact that we are only considering a single simulation in which our processing time realizations are randomly drawn from two exponential distributions. Taking the average over multiple simulations, say more than 10.000 , resolves this issue.

\subsubsection{Computational experience}

In this section, we present computational results to study the performance of the policies in Sections 5.3.1 and 5.3.2 with respect to the optimal policy in a stochastic scheduling setting. All computations are performed in MATLAB. First, we present a conjecture on the performance of all scheduling policies.

Conjecture 5.3.5. For the stochastic scheduling problem with only a single job in both classes, no policy can perform better than partitioning the two jobs evenly over the machine.

This leads to a conjectured lower bound on the performance guarantee of all scheduling policies. For the stochastic scheduling problem with $n_{1}=1$ and $n_{2}=1$, we have

$$
\mathbb{E}\left[\Pi^{E Q U I}(1,1, \alpha)\right]=2 \mathbb{E}\left[\min \left\{X_{1}, X_{2}\right\}\right]+\left(\mathbb{E}\left[X_{1}\right]+\mathbb{E}\left[X_{2}\right]\right)=\frac{2 \alpha}{1+\alpha}+(1+\alpha),
$$

where the terms refer to the expected completion times of the first and second job that finish. Furthermore, assume without loss of generality that $\mathbb{E}\left[X_{1}\right]>\mathbb{E}\left[X_{2}\right]$. Then,

$$
\mathbb{E}\left[\Pi^{*}(1,1, \alpha)\right]=2 \mathbb{E}\left[X_{2}\right]+\mathbb{E}\left[X_{1}\right]=2 \alpha+1 .
$$

Combining the previous two expressions, we obtain

$$
\max _{\substack{0<\alpha<1 ; \\ n_{1}, n_{2} \geq 1}}\left\{\frac{\mathbb{E}\left[\Pi\left(n_{1}, n_{2}, \alpha\right)\right]}{\mathbb{E}\left[\Pi^{*}\left(n_{1}, n_{2}, \alpha\right)\right]}\right\} \geq \max _{0<\alpha<1}\left\{\frac{\frac{2 \alpha}{1+\alpha}+(1+\alpha)}{2 \alpha+1}\right\}=6-2 \sqrt{6} \approx 1.10102 .
$$

for every possible scheduling policy П. Here, the second equality follows from the fact that the maximum is reached at $\alpha=(\sqrt{6}-1) / 5 \approx 0.28989$.

In our computations, we compare the values of PCC, $k$-PCC, $k$-APCC, CEQUI, $k$-CEQUI, and PLEARN with the optimal solution, which is equivalent to SEPT. Note that we do not consider the values of EQUI, since it follows from Lemma 5.3.1 that its performance is identical to PCC. The scheduling instances studied are as follows: for each job class, we generate 20 different numbers of jobs by using the uniform distribution $U(1,100)$. Furthermore, we set $\lambda_{1}$ equal to 1 and let $\lambda_{2}$ be an element of $\{0.1,0.2, \ldots, 1.0\}$. This gives a total of 4000 different 
computations covering the majority of interesting job class settings, i.e., the cases in which both job classes have either many or few number of jobs, and the mixed case in which some class has many and some other one has just a few number of jobs.

In our computations, 10000 simulations are run for each scheduling instance. In each of those simulations, we draw for class $J_{i}$ a total of $n_{i}$ processing time realizations from the exponential distribution with parameter $\lambda_{i}$. Using these realizations the sum of completion times for each of the policies is calculated. Performance of the policies PCC, $k$-PCC, $k$-APCC, CEQUI, $k$-CEQUI, and PLEARN is measured by taking the average objective value of the policy over the average objective value of OPT, which is an estimate for the expected sum of completion times of OPT,

$$
\mathbb{E}\left[\Pi^{*}\left(n_{1}, n_{2}, \alpha\right)\right]=\frac{n_{1}\left(n_{1}+1\right)}{2}+\frac{n_{2}\left(n_{2}+1\right)}{2} \alpha+n_{1} n_{2} \alpha .
$$

Here, $n_{1}, n_{2}$, and $\alpha$ depend on the scheduling instance. Since this latter value is much faster to calculate, this is used in our computations.

Before running our simulations, we need to decide which values of $k$ will be used for policies $k$-PCC, $k$-APCC, and $k$-CEQUI, and which $\beta_{1}$ and $\beta_{2}$ are chosen for policy PLEARN. In order to do this, we run preliminary computations on a small set of arbitrarily chosen scheduling instances. The best performance for the first two policies is obtained when $k$ is close to the minimum of $\sqrt{n_{1}}$ and $\sqrt{n_{2}}$. Apparently, learning with $\sqrt{n_{i}}$ jobs for class $J_{i}$ strikes a good balance between getting information about the true value of the parameter and postponing decision making with the resulting higher costs in terms of the waiting times. Hence, we set $k=\min \left\{k_{1}, k_{2}\right\}$ with $k_{i}=\left\lfloor\sqrt{n_{i}}\right\rfloor$ for the $k$-APCC policy. The computations also confirm Conjecture 5.3.4 that it is always better to learn with an odd number of jobs in case of policy $k$-PCC. For this policy, we therefore set $k$ as above and add a value of one to $k_{i}$ in case this number is even.

For $k$-CEQUI, we again set $k=\min \left\{k_{1}, k_{2}\right\}$, but this time with $k_{i}=\left\lfloor\zeta \cdot n_{i}\right\rfloor$, where $\zeta$ is chosen in order to obtain a good overall performance of the policy. It turns out that the best choice for $\zeta$ is given by 1.5. Intuitively, we want to avoid taking $k$ too small, since this increases the probability of a "wrong" decision with high waiting times as a result. Lastly, we need to decide on $\beta_{1}$ and $\beta_{2}$ for the PLEARN policy. First, we set $\beta_{i}=1+\xi \cdot c_{i}$, where $\xi$ is, just like $\zeta$, chosen to obtain a reasonable performance of the policy. Then, we find that a small number of jobs requires a fast learning scheme, that is, a relatively high $\beta_{i}$, while a high number of jobs requires the opposite. It further follows that in case the difference between $n_{1}$ and $n_{2}$ is very large, it is also better to keep $\beta_{i}$ relatively low and in that way adhere to a slow learning scheme. This leads to the following choice for $\xi$ : if $n_{1}<30$ and $n_{2}<30$, then $\xi=\min \left(n_{1} / n_{2} ; n_{2} / n_{1}\right)+0.5$; otherwise, $\xi=\max \left(\min \left(n_{1} / n_{2} ; n_{2} / n_{1}\right) \cdot 0.25 ; 0.1\right)$.

The computational results are divided into three parts depending on the number of jobs in both classes: (A) $n_{1}<n_{2}$, (B) $n_{1}>n_{2}$, and (C) $n_{1}=n_{2}$. For each of these parts, we present for each policy the performance guarantees over all scheduling instances in a box-and-whisker plot. Thereby, we distinguish between the instances corresponding to $\alpha=0.1$ up to $\alpha=1.0$. All boxplots are given in Figures 5.2, 5.3, and 5.4 of Appendix 5.5.2. The line in the middle of the box indicates the median, the bottom and top of the box denote the 25th and 75th percentile, and the ends of the whiskers represent the minimum and maximum values.

Let us start with part (A), i.e., $n_{1}<n_{2}$. In Figure 5.2, we can see that PCC performs the 
worst of all policies, with a maximum performance guarantee of 1.2069 attained at $\alpha=0.2$ and $n_{1} / n_{2}=37 / 77=0.48$. Note that this is in full agreement with the worst case ratio found in Theorem 5.3.2. The wide dispersion of the performance guarantees of PCC is explained by the fact that learning with only one job is not enough when $n_{1}$ is large. In that case, more learning jobs are needed, which is shown by the lower performance guarantees of $k$-PCC compared to PCC. Learning by using the average processing times over $k$ jobs of each class, as policy $k$-APCC, also does not lead to a significant improvement over $k$-PCC. The only policies that perform better are the policies based on EQUI: the maximum performance guarantees of CEQUI and $k$-CEQUI are much lower than the ones from $k$-PCC. However, this does not hold for their median values. Reason is that $k$-PCC performs very bad when $n_{1}<6$, while it gives low performance guarantees compared to CEQUI and $k$-CEQUI in all other cases. Overall, the best results are obtained by the PLEARN policy, with a maximum performance guarantee of 1.1096 at $\alpha=0.3$. This is very close to the conjectured lower bound on all scheduling policies in (5.6).

In part (B) with $n_{1}>n_{2}$, we observe slightly different computational results than in part (A). That is, all policies have much lower maximum performance guarantees. This is explained by the relative small number of jobs in class $J_{2}$, with the lowest expected processing time, such that a "wrong" decision by a policy has a smaller impact on the performance guarantee than in the previous part. Another difference lies in the observation that the best performance is obtained by $k$-PCC, with a maximum of 1.1161 at $\alpha=0.4$. Moreover, the best performing policy in part (A) is now the worst one besides PCC. However, PLEARN still gives a maximum performance guarantee of only 1.1197. Overall, we therefore have for this part that policies $k$-PCC, CEQUI, $k$-CEQUI, and PLEARN are all either below or very close to the value of the conjectured lower bound on all scheduling policies in (5.6).

The last part to be discussed is the one with $n_{1}=n_{2}$, that is, part (C). Not only do all policies in this part have a much smaller dispersion of the performance guarantees than in the previous two parts (especially CEQUI and $k$-CEQUI), there is also a clear order in terms of the maximum performance guarantees. The worst performing policy is PCC, followed by $k$-APCC, $k$-PCC, the EQUI based policies CEQUI and $k$-CEQUI, and the best policy PLEARN with a maximum performance guarantee of 1.1091 at $\alpha=0.4$. Remark that this value is again, like in parts (A) and (B), very close to the conjectured lower bound in (5.6). Finally, we observe that the maximum performance guarantee of PCC, i.e., 1.1710 at $\alpha=0.2$, is very close to 1.1716, and as such confirming our worst case ratio in Theorem 5.3.2.

To conclude, the performance of PLEARN seems to be the best over all three parts, especially taking into account the fact that the scheduler does not know if he/she is in part (A) or in part (B) when $n_{1} \neq n_{2}$ (there is no knowledge on the parameter values of the processing time distributions). Moreover, we expect that the performance guarantees of PLEARN could possibly be lowered even more by choosing the $\beta_{1}$ and $\beta_{2}$ values in a more sophisticated way, depending on the initial number of jobs in each class.

\subsection{Concluding remarks}

This chapter studies the theoretical and empirical performance of several policies for a stochastic single machine scheduling problem with incomplete information. The jobs are divided over 
two different job classes and the incompleteness lies in the fact that the parameters of the processing time distributions of these classes are unknown. Note that the Shortest Expected Processing Time (SEPT) policy is no longer applicable in this case. We consider two different problem settings: without and with preemption of jobs. For the first problem setting, we introduce deterministic and randomized policies, based on the following idea of Class Comparison: schedule one job from each class, compare the resulting processing times, and continue by processing the jobs of the class corresponding to the faster job followed by the jobs of the other class. Further, we show that these scheduling policies have tight performance guarantees. For the second problem setting, we conjecture that there is a constant lower bound on the performance guarantee of all possible scheduling policies. In addition, we provide tight performance guarantees for the EQUI-partition policy and a preemptive version of the Class Comparison policies. Finally, we introduce several learning policies for which we do not have any performance guarantees, and we compare the computational performance of all proposed scheduling policies. We find that our PLEARN policy outperforms all other policies on almost all instances. Moreover, we claim that the performance of PLEARN could possibly be improved by choosing the $\beta_{1}$ and $\beta_{2}$ values in a more sophisticated way. We remark that none of the learning policies explicitly uses the fact that the processing times are exponentially distributed. Therefore, we expect that these policies will also perform reasonably on more general instances. 


\subsection{Appendix}

\subsubsection{Preliminaries on probability theory}

In this section, we state several useful probabilities and conditional expectations. The first ones follow from a well-known feature of the exponential distribution: if $X_{1}$ and $X_{2}$ are both exponentially distributed with, respectively, parameters $\lambda_{1}$ and $\lambda_{2}$, then $\min \left\{X_{1}, X_{2}\right\}$ is also exponentially distributed with parameter $\lambda_{1}+\lambda_{2}$. As a direct consequence, we get

$$
\mathbb{E}\left[\min \left\{X_{1}, X_{2}\right\}\right]=\frac{1}{\lambda_{1}+\lambda_{2}}=\frac{\mathbb{E}\left[X_{1}\right] \mathbb{E}\left[X_{2}\right]}{\mathbb{E}\left[X_{1}\right]+\mathbb{E}\left[X_{2}\right]} .
$$

It also leads to the following two probabilities.

$$
\operatorname{Pr}\left[X_{1} \leq X_{2}\right]=\frac{\lambda_{1}}{\lambda_{1}+\lambda_{2}}=\frac{\mathbb{E}\left[X_{2}\right]}{\mathbb{E}\left[X_{1}\right]+\mathbb{E}\left[X_{2}\right]}
$$

and

$$
\operatorname{Pr}\left[X_{1}>X_{2}\right]=1-\frac{\lambda_{1}}{\lambda_{1}+\lambda_{2}}=\frac{\lambda_{2}}{\lambda_{1}+\lambda_{2}}=\frac{\mathbb{E}\left[X_{1}\right]}{\mathbb{E}\left[X_{1}\right]+\mathbb{E}\left[X_{2}\right]} .
$$

We can generalize the expectation in (5.7) by introducing two sets, i.e., $J_{1}=\left\{1, \ldots, n_{1}\right\}$ and $J_{2}=\left\{n_{1}+1, \ldots, n_{1}+n_{2}\right\}$, and letting $X^{j}$ denote a random variable for the processing time of job $j$, defined as follows:

$$
X^{j}=\left\{\begin{array}{lll}
X_{1}^{j} & \text { if } & j \in J_{1}, \\
X_{2}^{j} & \text { if } & j \in J_{2} .
\end{array}\right.
$$

In case $l=m$, we then obtain

$$
\mathbb{E}\left[\min \left\{X_{i}^{l}, X_{i}^{m}\right\}\right]=\mathbb{E}\left[X_{i}\right]
$$

while in case $l \neq m$, we find

$$
\mathbb{E}\left[\min \left\{X_{i}^{l}, X_{j}^{m}\right\}\right]= \begin{cases}\frac{1}{2} \mathbb{E}\left[X_{i}\right] & \text { if } i=j, \\ \mathbb{E}\left[X_{i}\right] \mathbb{E}\left[X_{j}\right] & \text { otherwise. }\end{cases}
$$

Further, we are interested in the expectation of $X_{1}$ given that $X_{1} \leq X_{2}$. That is,

$$
\begin{aligned}
\mathbb{E}\left[X_{1} \mid X_{1} \leq X_{2}\right] & =\frac{\int_{0}^{\infty} x_{1} \operatorname{Pr}\left[x_{1} \leq X_{2}\right] f_{X_{1}}\left(x_{1}\right) \partial x_{1}}{\int_{0}^{\infty} \operatorname{Pr}\left[x_{1} \leq X_{2}\right] f_{X_{1}}\left(x_{1}\right) \partial x_{1}}=\frac{\int_{0}^{\infty} x_{1} \exp \left(-\lambda_{2} x_{1}\right) \lambda_{1} \exp \left(-\lambda_{1} x_{1}\right) \partial x_{1}}{\int_{0}^{\infty} \exp \left(-\lambda_{2} x_{1}\right) \lambda_{1} \exp \left(-\lambda_{1} x_{1}\right) \partial x_{1}} \\
& =\frac{\lambda_{1} \int_{0}^{\infty} x_{1} \exp \left(-\left(\lambda_{1}+\lambda_{2}\right) x_{1}\right) \partial x_{1}}{\lambda_{1} \int_{0}^{\infty} \exp \left(-\left(\lambda_{1}+\lambda_{2}\right) x_{1}\right) \partial x_{1}}=\frac{1}{\lambda_{1}+\lambda_{2}}=\frac{\mathbb{E}\left[X_{1}\right] \mathbb{E}\left[X_{2}\right]}{\mathbb{E}\left[X_{1}\right]+\mathbb{E}\left[X_{2}\right]} .
\end{aligned}
$$

By similar calculations, we end up with the conditional expectations below.

$$
\begin{aligned}
& \mathbb{E}\left[X_{1} \mid X_{1}>X_{2}\right]=\frac{2 \lambda_{1}+\lambda_{2}}{\lambda_{1}\left(\lambda_{1}+\lambda_{2}\right)}=\frac{\mathbb{E}\left[X_{1}\right]\left(\mathbb{E}\left[X_{1}\right]+2 \mathbb{E}\left[X_{2}\right]\right)}{\mathbb{E}\left[X_{1}\right]+\mathbb{E}\left[X_{2}\right]}, \\
& \mathbb{E}\left[X_{2} \mid X_{2} \leq X_{1}\right]=\frac{1}{\lambda_{1}+\lambda_{2}}=\frac{\mathbb{E}\left[X_{1}\right] \mathbb{E}\left[X_{2}\right]}{\mathbb{E}\left[X_{1}\right]+\mathbb{E}\left[X_{2}\right]}
\end{aligned}
$$


and

$$
\mathbb{E}\left[X_{2} \mid X_{2}>X_{1}\right]=\frac{\lambda_{1}+2 \lambda_{2}}{\lambda_{2}\left(\lambda_{1}+\lambda_{2}\right)}=\frac{\mathbb{E}\left[X_{2}\right]\left(2 \mathbb{E}\left[X_{1}\right]+\mathbb{E}\left[X_{2}\right]\right)}{\mathbb{E}\left[X_{1}\right]+\mathbb{E}\left[X_{2}\right]}
$$

Note that the expectation of the processing time of a job of class $J_{i}$ can be denoted by using all the probabilities and conditional expectations given above,

$$
\mathbb{E}\left[X_{i}\right]=\operatorname{Pr}\left[X_{i} \leq X_{j}\right] \mathbb{E}\left[X_{i} \mid X_{i} \leq X_{j}\right]+\operatorname{Pr}\left[X_{i}>X_{j}\right] \mathbb{E}\left[X_{i} \mid X_{i}>X_{j}\right],
$$

for $i \neq j$. Another notion is related to the memoryless property of the exponential distribution.

$$
\begin{aligned}
\mathbb{E}\left[X_{i}-X_{j} \mid X_{i}>X_{j}\right] & =\mathbb{E}\left[X_{i} \mid X_{i}>X_{j}\right]-\mathbb{E}\left[X_{j} \mid X_{i}>X_{j}\right] \\
& =\frac{\mathbb{E}\left[X_{i}\right]\left(\mathbb{E}\left[X_{i}\right]+2 \mathbb{E}\left[X_{j}\right]\right)}{\mathbb{E}\left[X_{i}\right]+\mathbb{E}\left[X_{j}\right]}-\frac{\mathbb{E}\left[X_{i}\right] \mathbb{E}\left[X_{j}\right]}{\mathbb{E}\left[X_{i}\right]+\mathbb{E}\left[X_{j}\right]} \\
& =\frac{\left.\mathbb{E}\left[X_{i}\right]^{2}+\mathbb{E}\left[X_{i}\right] \mathbb{E}\left[X_{j}\right]\right)}{\mathbb{E}\left[X_{i}\right]+\mathbb{E}\left[X_{j}\right]}=\mathbb{E}\left[X_{i}\right] .
\end{aligned}
$$

\subsubsection{Figures of computational results}

The computational results of Section 5.3.3 are divided into three parts depending on the number of jobs in each class: (A) $n_{1}<n_{2}$, (B) $n_{1}>n_{2}$, and (C) $n_{1}=n_{2}$. For each of these parts, we present the performance guarantees for the policies PCC, $k$-PCC, $k$-APCC, CEQUI, $k$-CEQUI, and PLEARN, over all scheduling instances. Thereby, we distinguish between the instances corresponding to $\alpha=0.1$ up to $\alpha=1.0$. This is all done on the next three pages in box-andwhisker plots. The line in the middle of the boxes indicates the median, the bottom and top of the boxes denote the 25th and 75th percentile, and the ends of the whiskers represent the minimum and maximum values. 


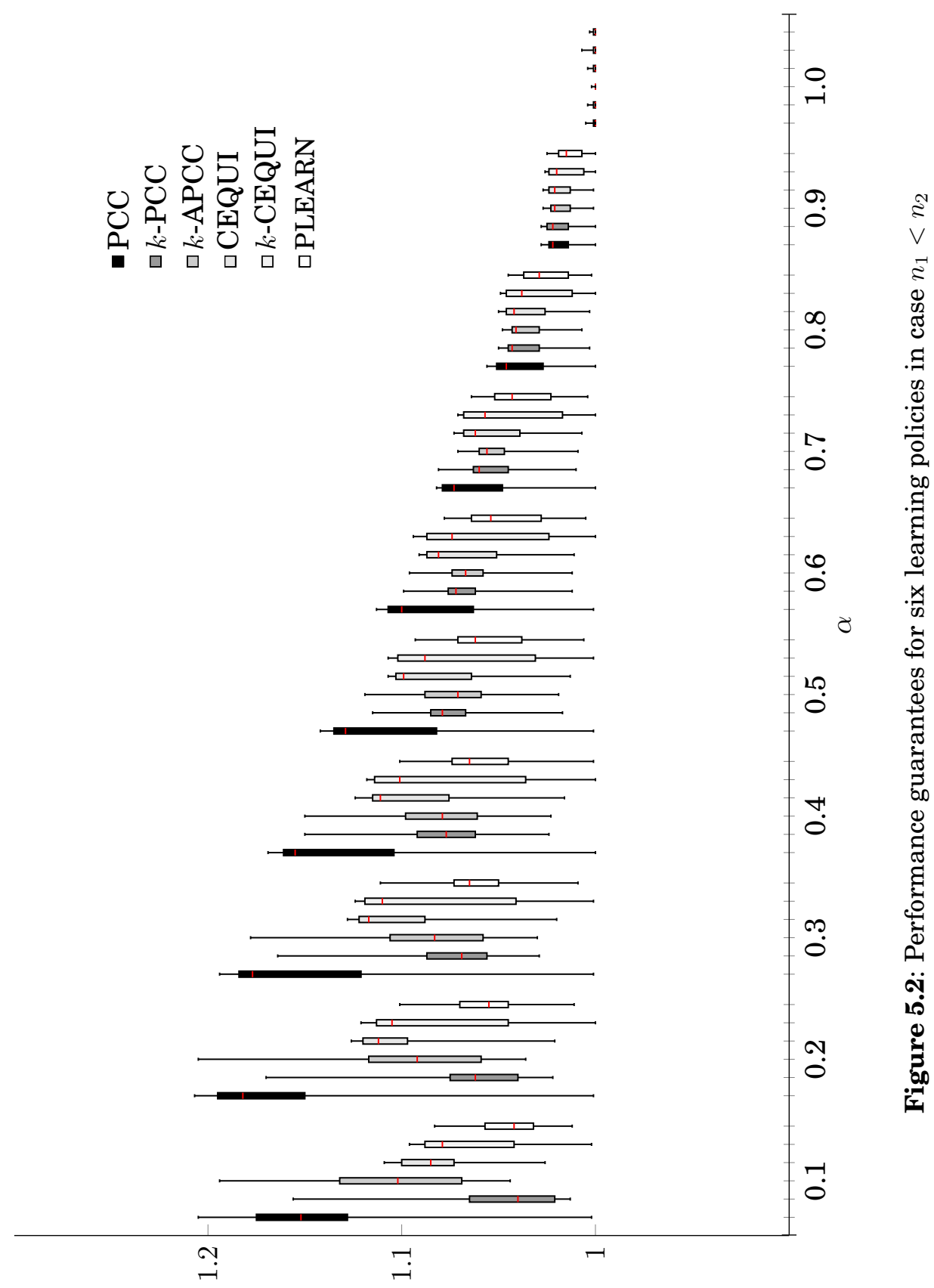




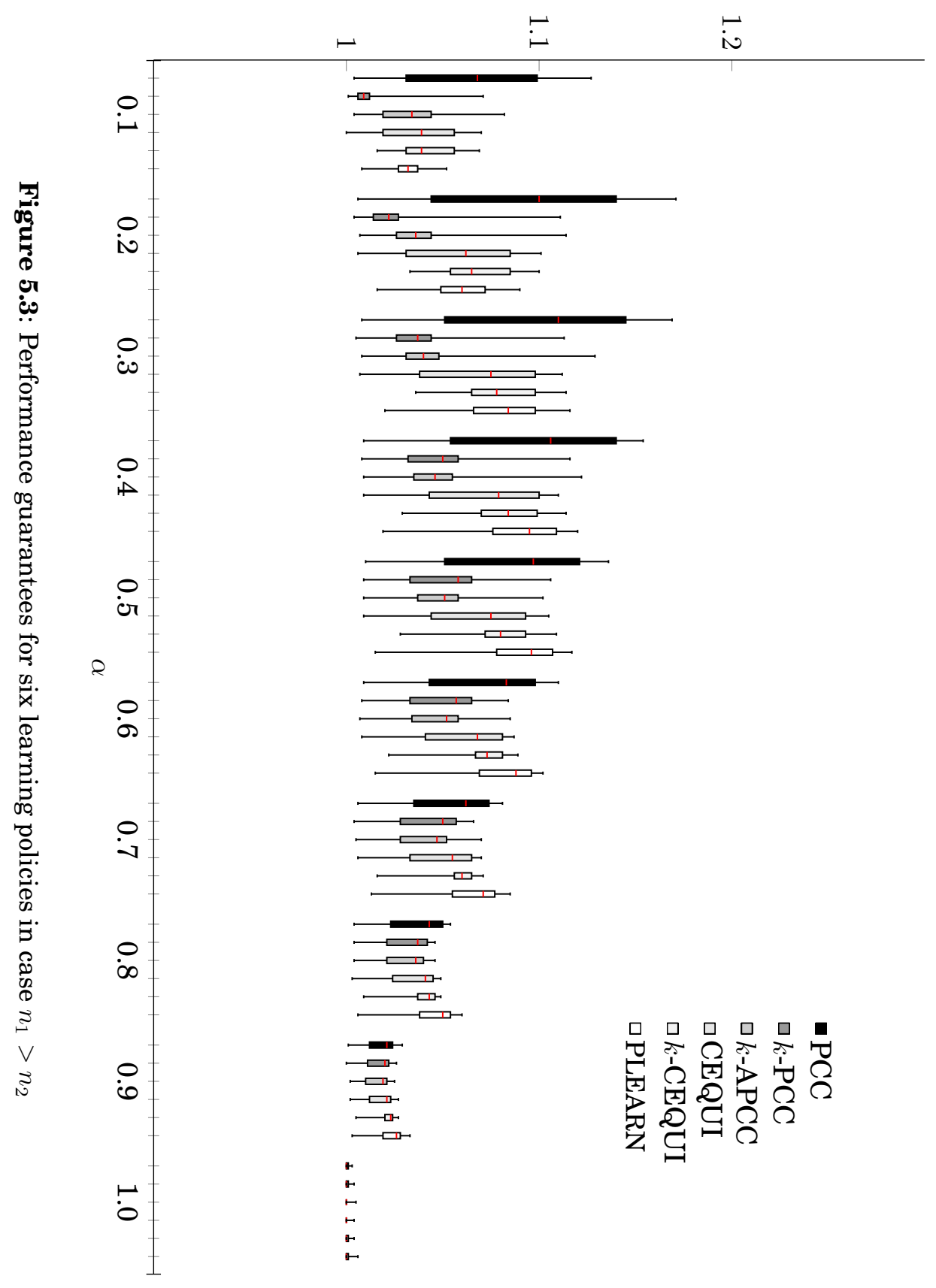




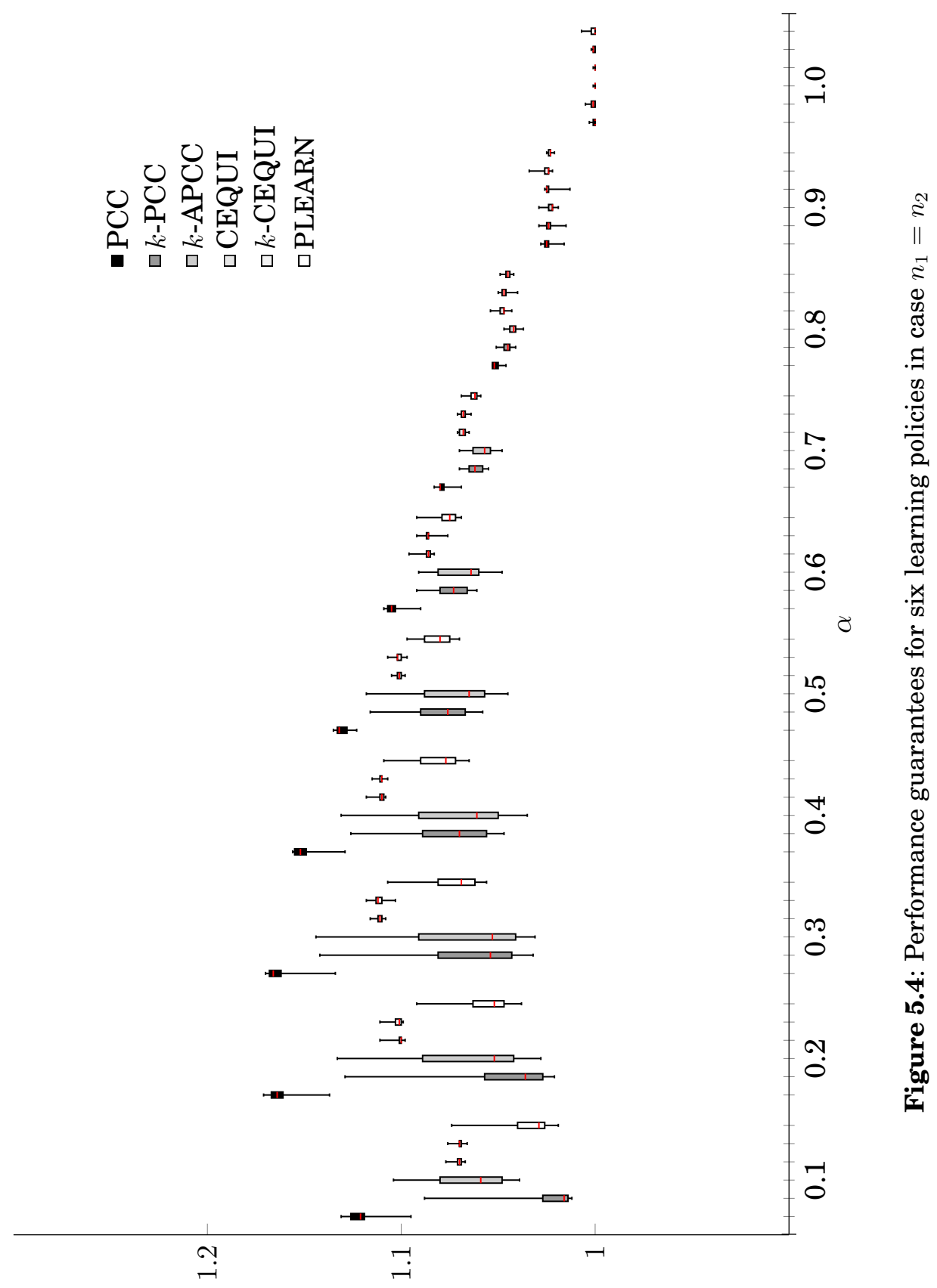




\section{Bibliography}

[1] N. Abramson. The ALOHA system: another alternative for computer communications. In Proceedings of AFIPS 1970 (Fall), pages 281-285, 1970.

[2] Y. Akcay, H.P. Natarajan, and S.H. Xu. Joint dynamic pricing of multiple perishable products under consumer choice. Management Science, 56(8):1345-1361, 2010.

[3] H. Akhlaghpour, M. Ghodsi, N. Haghpanah, H. Mahini, V.S. Mirrokni, and A. Nikzad. Optimal iterative pricing over social networks. In WINE'10, pages 415-423, Berlin, Heidelberg, 2010. Springer-Verlag.

[4] J.A. Aloysius. Research joint ventures: a cooperative game for competitors. European Journal of Operational Research, 136(3):591 - 602, 2002.

[5] A. Aram, C. Singh, S. Sarkar, and A. Kumar. Cooperative profit sharing in coalition based resource allocation in wireless networks. In Proceedings of INFOCOM 2009, IEEE, pages 2123-2131, 2009.

[6] V.F. Araman and R. Caldentey. Dynamic pricing for nonperishable products with demand learning. Operations Research, 57(5):1169-1188, 2009.

[7] T. Aydinliyim and G.L. Vairaktarakis. Coordination of outsourced operations to minimize weighted flow time and capacity booking costs. Manufacturing \& Service Operations Management, 12(2):236-255, 2010.

[8] K.S. Azoury. Bayes solution to dynamic inventory models under unknown demand distribution. Management Science, 31(9):1150-1160, 1985.

[9] N. Bansal and K. Pruhs. Server scheduling in the lp norm: a rising tide lifts all boat. In STOC '03: Proceedings of the thirty-fifth annual ACM symposium on Theory of computing, pages 242-250, New York, NY, USA, 2003. ACM.

[10] E.M.L. Beale. On minimizing a convex function subject to linear inequalities. Journal of the Royal Statistical Society. Series B (Methodological), 17(2):173-184, 1955.

[11] L. Becchetti and S. Leonardi. Nonclairvoyant scheduling to minimize the total flow time on single and parallel machines. Journal of the ACM, 51(4):517-539, 2004. 
[12] R. E. Bellman and L. A. Zadeh. Decision-making in a fuzzy environment. Management Science, 17(4):141-161, 1970.

[13] R.E. Bellman. Dynamic Programming. Princeton University Press, Princeton, 1957.

[14] P. Belobaba. Airline yield management: an overview of seat inventory control. Transportation Science, 21(2):63-73, 1987.

[15] P. Belobaba. Application of a probabilistic decision model to airline seat inventory control. Operations Research, 37(2):183-197, 1989.

[16] G. Bergantiños and J. Vidal-Puga. The optimistic $T U$ game in minimum cost spanning tree problems. International Journal of Game Theory, 36(2):223-239, 2007.

[17] A. Berger, A. Grigoriev, and J Van Loon. Price strategy implementation. Computers \& Operations Research, 38(2):420 - 426, 2011.

[18] G. Bitran and R. Caldentey. An overview of pricing models for revenue management. Manufacturing and Service Operations Management, 5(3):203-229, 2002.

[19] S. Boztas. On transmission scheduling in wireless networks. In Proceedings of Information Theory, IEEE International Symposium, pages 2754-2758, 2006.

[20] C. Briand, H. La, and J. Erschler. A robust approach for the single machine scheduling problem. Journal of Scheduling, 10(3):209-221, 2007.

[21] A.N. Burnetas and M.N. Katehakis. On sequencing two types of tasks on a single processor under incomplete information. Probability in the Engineering and Informational Sciences, 7(1):85-119, 1993.

[22] P. Calleja, A. Estévez-Fernández, P. Borm, and H. Hamers. Job scheduling, cooperation, and control. Operations Research Letters, 34(1):22 - 28, 2006.

[23] Y. Cao and V.O.K. Li. Scheduling algorithms in broadband wireless networks. Proceedings of the IEEE, 89(1):76 -87, 2001.

[24] L.M.A. Chan, Z.J. Shen, D. Simchi-Levi, and J.L. Swann. Handbook of quantitative supply chain analysis: Modeling in the e-business era, chapter 9, pages 335-382. Kluwer Academic Publishers, Boston/Dordrecht/London, 2004.

[25] L.M.A. Chan, D. Simchi-Levi, and J. Swann. Pricing, production, and inventory policies for manufacturing with stochastic demand and discretionary sales. Manufacturing and Service Operations Management, 8(2):149-168, 2006.

[26] L. Chen and E.L. Plambeck. Dynamic inventory management with learning about the demand distribution and substitution probability. Manufacturing \& Service Operations Management, 10(2):236-256, 2008.

[27] C. Chung, T. Nonner, and A. Souza. SRPT is 1.86-competitive for completion time scheduling. In Proceedings of the 21st Annual ACM-SIAM Symposium on Discrete Algorithms (SODA) 2010, pages 1373-1388, 2010. 
[28] R. Cole, J.R. Correa, V. Gkatzelis, V. Mirrokni, and N. Olver. Inner product spaces for minsum coordination mechanisms. In Proceedings of the 43rd ACM Symposium on Theory of Computing (STOC), pages 539-548, 2011.

[29] S. Conti, H. Held, M. Pach, M. Rumpf, and R. Schultz. Shape optimization under uncertainty: A stochastic programming perspective. SIAM Journal on Optimization, 19(4):1610-1632, 2009.

[30] E. Cope. Bayesian strategies for dynamic pricing in e-commerce. Naval Research Logistics, 54(3):265-281, 2006.

[31] I. Curiel, G. Pederzoli, and S. Tijs. Sequencing games. European Journal of Operational Research, 40(3):344-351, 1989.

[32] S. Damodaran and K.M. Sivalingam. Scheduling in wireless networks with multiple transmission channels. In Proceedings of the 7th International Conference on Network Protocols (ICNP '99), pages 262 - 269, 1999.

[33] G.B. Dantzig. Maximization of a Linear Function of Variables Subject to Linear Inequalities, in Activity Analysis of Production and Allocation. Wiley, N.Y., 1951.

[34] G.B. Dantzig. Linear programming under uncertainty. Management Science, 1(3/4):197-206, 1955.

[35] B.C. Dean. Approximation Algorithms for Stochastic Scheduling Problems. PhD thesis, Massachusetts Institute of Technology, 2005.

[36] M.H. DeGroot. Optimal Statistical Decisions. McGraw-Hill, New York, 1970.

[37] E. Delage and Y. Ye. Distributionally robust optimization under moment uncertainty with application to data-driven problems. Operations Research, 58(3):595-612, 2010.

[38] X. Deng, N. Gu, T. Brecht, and K. Lu. Preemptive scheduling of parallel jobs on multiprocessors. SIAM Journal on Computing, 30(1):145-160, 2000.

[39] B. Dutta and A. Kar. Cost monotonicity, consistency and minimum cost spanning tree games. Games and Economic Behavior, 48(2):223 - 248, 2004.

[40] J. Edmonds. Scheduling in the dark. Theoretical Computer Science, 235(1):109 - 141, 2000.

[41] J. Edmonds, D. Chinn, T. Brecht, and X. Deng. Non-clairvoyant multiprocessor scheduling of jobs with changing execution characteristics. Journal of Scheduling, 6(3):231$250,2003$.

[42] S. Esmaeilsabzali and N.A. Day. Online pricing for web service providers. In EDSER '06: Proceedings of the 2006 International Workshop on Economics Driven Software Engineering Research, pages 37-42, New York, NY, USA, 2006. ACM.

[43] F.F. Farias and B. Van Roy. Dynamic pricing with a prior on market response. Operations Research, 58(1):16-29, 2010. 
[44] A. Fiat and G.J. Woeginger. Online Algorithms: The State of the Art. Springer-Verlag, Berlin, 1998.

[45] M.R. Garey and D.S. Johnson. Computers and Intractability: A guide to the theory of NP-completeness. W.H. Freeman \& Co, New York, 1979.

[46] D.B. Gillies. Some theorems on n-person games. Dissertation, Princeton University, Department of Mathematics, 1953.

[47] J.C. Gittins. Bandit processes and dynamic allocation indices (with discussion). Journal of the Royal Statistical Society, Series B (Methodological), 41(2):148-177, 1979.

[48] J.C. Gittins. Multi-armed bandit allocation indices. Wiley, N.Y., 1989.

[49] J.C. Gittins and K.D. Glazebrook. On Bayesian models in stochastic scheduling. Journal of Applied Probability, 14(3):556-565, 1977.

[50] J.C. Gittins and D.M. Jones. A dynamic allocation index for the sequential design of experiments. In Progress in Statistics, pages 241-266. 1974.

[51] K.D. Glazebrook and R.W. Owen. On the value of adaptive solutions to stochastic scheduling problems. Mathematics of Operations Research, 20(1):65-89, 1995.

[52] V. Guruswami, J.D. Hartline, A.R. Karlin, and D. Kempe. On profit-maximizing envyfree pricing. In SODA '05: Proceedings of the 16th Annual ACM-SIAM Symposium on Discrete Algorithms, pages 1164-1173, Philadelphia, PA, USA, 2005. Society for Industrial and Applied Mathematics.

[53] T. Hamada and K.D. Glazebrook. A Bayesian sequential single machine scheduling problem to minimize the expected weighted sum of flowtimes of jobs with exponential processing times. Operations Research, 41(5):924-934, 1993.

[54] T. Hamada and M. Tamaki. Some results on a Bayesian sequential scheduling on two identical parallel processors. Journal of the Operations Research Society of Japan, 42(14):316-329, 1999.

[55] H. Hamers, J. Suijs, S.H. Tijs, and P. Borm. The split core for sequencing games. Games and Economic Behaviour, 15(2):165-176, 1996.

[56] B. Hartman and M. Dror. Optimizing centralized inventory operations in a cooperative game theory setting. IIE Transactions, 35(3):243-257, 2003.

[57] T. Ichiishi. Super-modularity: applications to convex games and the greedy algorithm for LP. Journal of Economic Theory, 25(2):283-286, 1981.

[58] B. Kalyanasundaram and K. Pruhs. Speed is as powerful as clairvoyance. Journal of the ACM, 47(4):617-643, 2000.

[59] E. Kemahlioglu-Ziya and III Bartholdi, J.J. Centralizing inventory in supply chains by using shapley value to allocate the profits. Manufacturing \& Service Operations Management, page (Accepted for publication), 2011. 
[60] L. Kleinrock and F. Tobagi. Packet switching in radio channels: Part i-carrier sense multiple-access modes and their throughput-delay characteristics. IEEE Transactions on Communications, 23(12):1400 - 1416, 1975.

[61] R. Kohli and H. Park. A cooperative game theory model of quantity discounts. Management Science, 35(6):693-707, 1989.

[62] P.K. Kopalle, A.G. Rao, and J.L. Assunção. Asymmetric reference price effects and dynamic pricing policies. Marketing Science, 15(1):60-85, 1996.

[63] P. Kouvelis and G. Yu. Robust Discrete Optimization and Its Applications. Kluwer Academic Publishers, Boston, 1997.

[64] R. La and V. Anantharam. A game-theoretic look at the gaussian multiaccess channel. In Proceedings of the March 2003 DIMACS Workshop on Network Information Theory, pages 87-106, 2004.

[65] M.A. Lariviere and E.L. Porteus. Stalking information: Bayesian inventory management with unobserved lost sales. Management Science, 45(3):346-363, 1999.

[66] J.Y-T. Leung. Handbook of scheduling: algorithms, models, and performance analysis. Chapman \& Hall/CRC, N.Y., 2004.

[67] S.-Y.R. Li, R.W. Yeung, and C. Ning. Linear network coding. IEEE Transactions on Information Theory, 49(2):371-381, 2003.

[68] K.Y. Lin. Dynamic pricing with real-time demand learning. Operations Research, 174(1):522-538, 2003.

[69] K.Y. Lin. Dynamic pricing with real-time demand learning. European Journal of Operational Research, 174(1):522 - 538, 2006.

[70] K. Littlewood. Journal of Revenue and Pricing Management, 4(2):111-123, 2005.

[71] X. Lu, J.-S. Song, and K. Zhu. Inventory control with unobservable lost sales and bayesian updates. Working paper, 2006.

[72] I.J. Lustig. In his own voice - interview with george b. dantzig, 2000. http://www2.informs.org/History/dantzig/index.htm.

[73] C. Maglaras and J. Meissner. Dynamic pricing strategies for multi-product revenue management problems. Manufacturing and Service Operations Management, 8(2):136$148,2006$.

[74] S. Mathur, L. Sankaranarayanan, and N.B. Mandayam. Coalitional games in gaussian interference channels. In Proceedings of Information Theory, IEEE International Symposium, pages 2210-2214, 2006.

[75] S. Mathur, L. Sankaranarayanan, and N.B. Mandayam. Coalitions in cooperative wireless networks. In Proceedings of Selected Areas in Communications, IEEE, pages 11041115, 2008. 
[76] N. Megow and T. Vredeveld. Approximation results for preemptive stochastic online scheduling. In Y. Azar and T. Erlebach (eds): Proceedings of the 14th European Symposium on Algorithms (ESA 2006), pages 516-527, 2006. Lecture Notes in Computer Science; Vol. 4168.

[77] R. H. Möhring, F. J. Radermacher, and G. Weiss. Stochastic scheduling problems I: General strategies. ZOR - Zeitschrift für Operations Research, 28:193-260, 1984.

[78] R.H. Möhring, A.S. Schulz, and M. Uetz. Approximation in stochastic scheduling:the power of LP-based priority policies. Journal of ACM, 46(6):924-942, 1999.

[79] G.E. Monahan, N.C. Petruzzi, and W. Zhao. The dynamic pricing problem from a newsvendor's perspective. Manufacturing and Service Operations Management, 6(1):73-91, 2004.

[80] T. Moscibroda, Y. A. Oswald, and R. Wattenhofer. How optimal are wireless scheduling protocols? In Proceedings of the 26th Annual IEEE Conference on Computer Communications (INFOCOM), pages 1433-1441, 2007.

[81] R. Motwani, S. Phillips, and E. Torng. Nonclairvoyant scheduling. Theoretical Computer Science, 130(1):17 - 47, 1994.

[82] M. Nagarajan and G. Sosic. Game-theoretic analysis of cooperation among supply chain agents: review and extensions. European Journal of Operational Research, 187(3):719 $-745,2008$.

[83] U. Özen, J. Fransoo, H. Norde, and M. Slikker. Cooperation between multiple newsvendors with warehouses. Manufacturing \& Service Operations Management, 10(2):311$324,2008$.

[84] K. Pahlavan and P. Krishnamurthy. Principles of Wireless Networks: A Unified Approach. Prentice Hall PTR, 2001.

[85] D. Peidro, J. Mula, R. Poler, and F.-C. Lario. Quantitative models for supply chain planning under uncertainty: a review. The International Journal of Advanced Manufacturing Technology, 43(3):400-420, 2009.

[86] C. A. Phillips, C. Stein, and J. Wein. Minimizing average completion time in the presence of release dates. Mathematical Programming, 82:199 - 223, 1998.

[87] M. Pinedo. Stochastic scheduling with release dates and due dates. Operations Research, 31(3):559-572, 1983.

[88] M. Pinedo. Scheduling: Theory, Algorithms, and Systems. Springer, 2012.

[89] I. Popescu and Y. Wu. Dynamic pricing strategies with reference effects. Operations Research, 55(3):413-429, 2007.

[90] U. Rieder and J. Weishaupt. Customer scheduling with incomplete information. Probability in the Engineering and Informational Sciences, 9(2):269-284, 1995. 
[91] L. Roberts. ALOHA packet system with and without slots and capture. ACM SIGCOMM Computer Communication Review, 5(2):28-42, 1975.

[92] L.W. Robinson. A comment on gerchak and gupta's "on apportioning costs to customers in centralized continuous review inventory systems". Journal of Operations Management, 11(1):99 - 102, 1993.

[93] R. Rom and M. Sidi. Multiple access protocols. Springer-Verlag, New York, 1990.

[94] M.H. Rothkopf. Scheduling with random service times. Management Science, 12(9):703-713, 1966.

[95] M. Rothstein. An airline overbooking model. Transportation Science, 5(2):180-192, 1971.

[96] M. Rothstein. Hotel overbooking as a markovian sequential decision process. Decision Sciences, 5(3):389-404, 1974.

[97] W. Saad, Z. Han, M. Debbah, A. Hjø rungnes, and T. Başar. Linear network coding. IEEE Transactions on Information Theory, 49(2):371-381, 2003.

[98] H. Scarf. Bayes solutions of the statistical inventory problem. The Annals of Mathematical Statistics, 30(2):490-508, 1959.

[99] L Schrage. A proof of the optimality of the shortest remaining processing time discipline. Operations Research, 16:687 - 690, 1968.

[100] A. Schrijver. Combinatorial Optimization: Polyhedra and Efficiency. Springer-Verlag, N.Y., 2003.

[101] A.S. Schulz. New old algorithms for stochastic scheduling. In Algorithms for Optimization with Incomplete Information, 2005. Dagstuhl Seminar Proceedings, Vol. 05031.

[102] O. Shagdar, H. Yomo, T. Ohyama, R. Miura, and S. Obana. Transmission scheduling in multi-carrier multi-code spread aloha inter-vehicle communications system. In Proceedings of the 7th International Conference on Wireless On-demand Network Systems and Services (WONS), pages $66-73,2010$.

[103] L.S. Shapley. A value for n-person games. In H.W. Kuhn and A.W. Tucker, editors, Contributions to the Theory of Games II. Annals of Mathematics Study, pages 307-317. 1953.

[104] L.S. Shapley. Cores of convex games. International Journal of Game Theory, 1(1):11-26, 1971.

[105] R.A. Sitters. Competitive analysis of preemptive single-machine scheduling. Operations Research Letters, 38:585-588, 2010.

[106] W.E. Smith. Various optimizers for single stage production. Naval Research Logistics Quaterly, 3:59-66, 1956. 
[107] W. Stallings. Wireless Communications and Networks. Prentice Hall PTR, 2004.

[108] H. Takagi and L. Kleinrock. Optimal transmission ranges for randomly distributed packet radio terminals. IEEE Transactions on Communications, 32(3):246 - 257, 1984.

[109] S.H. Tijs. Bounds for the core and the $\tau$-value. In O. Moeschlin and D. Pallaschke, editors, Game Theory and Mathematical Economics, pages 123-132. 1981.

[110] A. Tucker and A. Gupta. Process control and scheduling issues for multiprogrammed shared-memory multiprocessors. ACM SIGOPS Operating Systems Review, 23(5):159_ $166,1989$.

[111] M. Uetz. Algorithms for deterministic and stochastic scheduling. PhD thesis, Technischen Universität Berlin, Berlin, Germany, 2002.

[112] L.R. Weatherford and S. Bodily. A taxonomy and research overview of perishable-asset revenue management: Yield management, overbooking, and pricing. Operations Research, 40(5):831-844, 1992.

[113] G. Weiss. Approximation results in parallel machines stochastic scheduling. Annals of Operations Research, 26(1):195-242, 1990.

[114] G. Weiss. Turnpike optimality of Smith's rule in parallel machines stochastic scheduling. Mathematics of Operations Research, 17(2):255-270, 1992.

[115] J. Yang and G. Yu. On the robust single machine scheduling problem. Journal of Combinatorial Optimization, 6(1):17-33, 2002.

[116] W. Zhao and Y.-S. Zheng. Optimal dynamic pricing for perishable assets with nonhomogeneous demand. Management Science, 46(3):375-388, 2000.

[117] M. Zorzi and S. Pupolin. Slotted aloha for high-capacity voice cellular communications. IEEE Transactions on Vehicular Technology, 43(4):1011-1021, 1994. 


\section{Nederlandse Samenvatting}

In dit proefschrift staan een aantal vraagstukken centraal uit het gebied van combinatorische optimalisering met onzekerheid. In de combinatorische optimalisering wordt onderzoek gedaan naar het vinden van de beste oplossing uit een beperkte selectie van mogelijke oplossingen. Hierbij wordt ervan uitgegaan dat degene die de beslissingen neemt volledige kennis heeft van alle relevante gegevens. Echter, zoals wijlen George Dantzig, bedenker van de simplexmethode [33], al benadrukte in een interview in het jaar 2000, is deze aanname niet altijd realistisch. In de meeste gevallen, zal de beste oplossing gevonden moeten worden terwijl men te maken heeft met onvolledige of zelfs niet beschikbare data.

In de afgelopen decennia is optimalisering onder onzekerheid uitgegroeid tot een belangrijk onderzoeksgebied. Hierbij ligt de focus op het nemen van beslissingen terwijl er onvolledige informatie beschikbaar is over de initiële gegevens of de eventuele gevolgen van de gemaakte beslissingen. In de praktijk is dit vaak het geval; men is meestal niet op de hoogte van alle relevante informatie en dit kan leiden tot het nemen van "foute" beslissingen. Het is daarom van groot belang om te onderzoeken hoe we om kunnen gaan met onzekerheid. Er is een breed scala aan methoden beschikbaar om het gebrek aan informatie in de combinatorische optimalisering te modelleren.

In een van de methoden om onzekerheid te modelleren wordt aangenomen dat een deel van de gegevens een bepaalde kansverdeling volgt. Dit model, genaamd stochastische optimalisering, wordt bestudeerd in de Hoofdstukken 2, 4 en 5. Het eerste van deze hoofdstukken, Hoofdstuk 2, is gewijd aan een dynamisch prijsprobleem voor een bedrijf dat een product verkoopt aan een groep potentiële klanten. Deze klanten kopen het product alleen als de prijs niet hoger is dan hun referentie prijs, dat wil zeggen, de maximale prijs die ze bereid zijn te betalen. Deze referentie prijs is echter tot op zekere hoogte onbekend bij het bedrijf en wordt daarom gemodelleerd als een stochastische variabele met een bepaalde kansverdeling. Het doel is om de prijzen per tijdseenheid zo vast te stellen dat de verwachte opbrengst van het bedrijf gemaximaliseerd wordt.

We bestuderen in dit hoofdstuk twee prijselasticiteitsmodellen. Voor het eerste model, met multiplicatieve vraagsverandering, geven we een optimaal algoritme met een looptijd die polynomiaal is in de input van het prijsprobleem. Het tweede model, met additieve vraagsverandering, blijkt aanzienlijk moeilijker om op te lossen. Voor dit probleem geven we daarom een algoritme met pseudo-polynomiale looptijd en we bewijzen het complementaire resultaat dat het probleem NP-moeilijk is. Bovendien presenteren we een FPTAS, een algo- 
ritme dat voor iedere $\epsilon>0$ gegarandeerd een oplossing vindt met een verwachte opbrengst die slechts een factor $(1+\epsilon)$ van de optimale verwachte opbrengst af ligt en een looptijd die polynomiaal is in de input van het probleem en $1 / \epsilon$.

In de andere twee hoofdstukken bestuderen we stochastische planningsproblemen, waarbij verschillende typen taken geplaatst moeten worden op een enkele machine. De behandelingstijden van deze taken zijn gemodelleerd als stochastische variabelen waarbij de parameters van de onderliggende kansverdelingen bekend zijn. Als het doel is om de som van de verwachte completeringstijden te minimaliseren, dan geeft de Kortste Verwachte Behandelingstijd (SEPT) regel de optimale oplossing [94]. Men kan zich echter afvragen hoe realistisch het is om volledige kennis over de parameters aan te nemen. Dit proefschrift introduceert daarom extra onzekerheid met betrekking tot de parameters en onderscheidt zich hiermee van de traditionele stochastische planningsliteratuur.

In Hoofdstuk 4 zijn de taken onderverdeeld in verschillende klassen. De behandelingstijd van iedere taak is exponentieel verdeeld met onbekende parameter, afhankelijk van de klasse waartoe de taak behoort. Het doel van het probleem is om de som van de verwachte completeringstijden te minimaliseren. Voor het modelleren van de onzekerheid rondom de parameters wordt een Bayesiaans raamwerk gebruikt, net als in het artikel van Hamada en Glazebrook [53]. Uit dit artikel blijkt dat in de meeste gevallen het vinden van de optimale oplossing van een Bayesiaans planningsprobleem zeer onpraktisch, zo niet onmogelijk, is. Daarom is het belangrijk om algoritmes te onderzoeken met weinig rekentijd en aantoonbaar goede prestaties. Deze algoritmes worden ook wel benaderingsalgoritmes genoemd. Enkele voorbeelden zijn de Kortste Verwachte Behandelingstijd (SEPT) regel en een aangepaste variant ( $\ell$-SEPT), die de verwachte behandelingstijd van de taken in een klasse iedere keer dat een taak uit die bepaalde klasse gerealiseerd wordt bijwerkt. In het hoofdstuk tonen we aan dat de prestaties van deze twee algoritmes niet ver afliggen van die van de optimale oplossing.

In Hoofdstuk 5 bestuderen we twee verschillende klassen van taken. Net als in het voorgaande hoofdstuk nemen we aan dat de behandelingstijd van iedere taak exponentieel verdeeld is met onbekende parameter, afhankelijk van de klasse waartoe de taak behoort. Dit maal is er echter totaal geen informatie beschikbaar over de parameters, zodat een Bayesiaans raamwerk niet toepasbaar is. Voor dit probleem, stellen wij een aantal benaderingsalgoritmes voor en we onderzoeken hun theoretische prestaties. Daarnaast vergelijken we de prestaties van deze algoritmes bij het bovenstaande probleem waarbij uitgevoerde taken tussentijds onderbroken mogen worden, in een experimentele opzet met een reeks andere algoritmes waarvoor wij geen garanties hebben ten aanzien van de kwaliteit. Alle onderzochte algoritmes worden aangeduid als "learning"-algoritmes, omdat ze proberen te leren over de werkelijke waardes van de parameters door gebruik te maken van de behandelingstijden van alle reeds gerealiseerde taken.

Een laatste benadering van onzekerheid binnen de combinatorische optimalisering wordt behandeld in Hoofdstuk 3 van dit proefschrift. Hierin bestuderen we een draadloos communicatie netwerk waarin elke knoop (antenne) voor zichzelf beslist wanneer zijn pakketten verzonden worden. Bij het ontwerpen van een draadloos netwerk is het belangrijk om de verzending van pakketten op een zodanige wijze te organiseren dat de prestatie van het netwerk optimaal is $[19,23,32,102]$. In het hierboven beschreven netwerk waarin elke knoop voor zichzelf beslist is dit specifiek van belang, omdat een gebrek aan coördinatie kan leiden tot onzekerheid over de ontvangst van de verzonden pakketten. Deze onzekerheid kan echter 
verholpen worden door samenwerking te initiëren tussen de verschillende knopen. De vraag die in dit hoofdstuk behandeld wordt is hoe de ontstane "winst" verdeelt moet worden onder de samenwerkende knopen.

In Hoofdstuk 3 definiëren en analyseren we daarvoor een coöperatief spel, een zogenaamd ALOHA spel. Daarnaast ontwikkelen we een aantal regels die aangeven hoe de winst verdeeld moet worden onder de knopen die met elkaar samenwerken. Deze regels worden aangeduid als Gewogen Winst Verdeling (WGS), omdat ze de grootte van de individuele bijdrage van elke knoop aan de behaalde winst meewegen. Verder bestuderen we drie oplossingsconcepten uit de coöperatieve speltheorie die worden toegepast als verdeelregels in het ALOHA spel: de Kern (geïntroduceerd door Gillies [46]), de Shapley waarde (geïntroduceerd door Shapley [103]) en de Compromis waarde (geïntroduceerd door Tijs [109]). Tot slot bekijken we hoe sensitief de bovenstaande verdeelregels zijn voor kleine veranderingen in het draadloze netwerk. 


\section{Curriculum Vitae}

Sebastián Marbán was born on May 16, 1984 in Heerlen, The Netherlands. In 2002, he received his Gymnasium diploma from the Bernardinuscollege in Heerlen. In September of the same year he started studying Econometrics with specialty Operations Research at Maastricht University. He received his Master's degree in September 2007. From September 2007 till August 2008, Sebastián was enrolled in the Master of Philosophy program Economic and Financial Research at Maastricht University. At the same time, he was employed as a teaching assistant. He received his second Master's degree in August 2008. In September 2008, he joined the Quantitative Economics department of Maastricht University as a PhD student and became part of the Operations Research group. The results of his research are presented in this thesis. 
